\title{
Neuropathology and Behavior after Short-term and Long-term Pinealectomy in the Rat.
}

\section{By}

Maxine De Butte, B.Sc., M.Sc.

\author{
A thesis submitted to the \\ Faculty of Graduate Studies and Research \\ in partial fulfillment of \\ the requirements for the degree of
}

\author{
Doctor of Philosophy \\ Psychology \\ (Specialization in Neuroscience)
}
Department of Psychology
and
Institute of Neuroscience

\author{
Carleton University \\ Ottawa, Ontario \\ December, 2004 \\ (C) 2004 Maxine De Butte
}




$\begin{array}{ll}\begin{array}{l}\text { Library and } \\ \text { Archives Canada }\end{array} & \begin{array}{l}\text { Bibliothèque et } \\ \text { Archives Canada }\end{array} \\ \begin{array}{l}\text { Published Heritage } \\ \text { Branch }\end{array} & \begin{array}{l}\text { Direction du } \\ \text { Patrimoine de l'édition }\end{array} \\ \begin{array}{l}\text { 395 Wellington Street } \\ \text { Ottawa ON K1A ON4 }\end{array} & \begin{array}{l}\text { 395, rue Wellington } \\ \text { Ottawa ON K1A ON4 } \\ \text { Canada }\end{array}\end{array}$

Your file Votre référence

ISBN: 0-494-00796-6

Ourfile Notre référence

ISBN: 0-494-00796-6

NOTICE:

The author has granted a nonexclusive license allowing Library and Archives Canada to reproduce, publish, archive, preserve, conserve, communicate to the public by telecommunication or on the Internet, loan, distribute and sell theses worldwide, for commercial or noncommercial purposes, in microform, paper, electronic and/or any other formats.

The author retains copyright ownership and moral rights in this thesis. Neither the thesis nor substantial extracts from it may be printed or otherwise reproduced without the author's permission.
AVIS:

L'auteur a accordé une licence non exclusive permettant à la Bibliothèque et Archives Canada de reproduire, publier, archiver, sauvegarder, conserver, transmettre au public par télécommunication ou par l'Internet, prêter, distribuer et vendre des thèses partout dans le monde, à des fins commerciales ou autres, sur support microforme, papier, électronique et/ou autres formats.

L'auteur conserve la propriété du droit d'auteur et des droits moraux qui protège cette thèse. $\mathrm{Ni}$ la thèse ni des extraits substantiels de celle-ci ne doivent être imprimés ou autrement reproduits sans son autorisation.
In compliance with the Canadian

Privacy Act some supporting forms may have been removed from this thesis.

While these forms may be included in the document page count, their removal does not represent any loss of content from the thesis.
Conformément à la loi canadienne sur la protection de la vie privée, quelques formulaires secondaires ont été enlevés de cette thèse.

Bien que ces formulaires aient inclus dans la pagination, il n'y aura aucun contenu manquant.

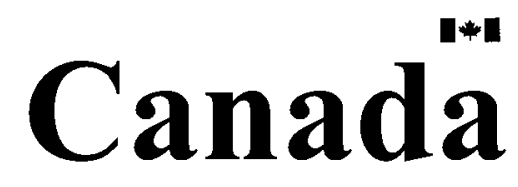




\section{ABSTRACT}

The pineal hormone melatonin not only regulates biological rhythms, but accumulating evidence suggests that it also acts as an antioxidant and stimulant of endogenous free radical scavenging systems. Melatonin levels decline with aging and even more so, in Alzheimer's disease. This experiment examined the effects of pinealectomy in the rat at different time points over a 20 month span using stereological neuron counts and neuropathological indices such as measures of cell death and markers of oxidative stress. Cognitive functioning was also assessed using the working memory version of the Morris water maze task and the elevated plus maze task. Since pinealectomy eliminates many neurohormones, some rats also received daily melatonin supplementation to determine if this would reverse the effects of pinealectomy. Animals were pinealectomized at 28 days of age and then were histologically examined at 2-month intervals up to 17 months of age. When tested at 2 months post surgery on the elevated plus maze, pinealectomized rats spent a greater proportion of time on the central hub compared to the sham rats. No other group differences were observed at this age nor were differences between the three groups found on any of this task's measures at 7 and 11 months post-surgery. At 15 months post-surgery, the melatonin-supplemented pinealectomized rats exhibited increased locomotor activity compared to the sham rats. Significant age effects were observed for all behavioral measures on the elevated plus maze task.

No significant impairment was observed on the morris water maze following 2,7 , or 11 months of pinealectomy. Age-related deficits in the Morris water maze were observed as 11-month rats performed more poorly than the 2-month rats. Pinealectomy caused a significant loss of hippocampal CA1 pyramidal cells between 2 and 4 months following 
surgery. Loss of cells in the CA3 was evident at 2 months post-pinealectomy. Melatonin replacement in the drinking water prevented these effects of pinealectomy. The results indicated that melatonin not only sustains the viability of hippocampal pyramidal cells but remarkably, may even promote their genesis in the adult rat. Biochemical analyses of hippocampal thiobarbituric acide reactive substance (TBARS) levels at 3 and 20 months post-surgery, revealed no significant differences between the groups. At 20 months of age, TBARS levels of the hippocampus were significantly elevated compared to that seen at 3 months of age. Additionally, biochemical analyses of hippocampal reduced glutathione, oxidized glutathione, and ratio of reduced glutathione to oxidized glutathione levels at 3 and 20 months post-surgery, revealed that the pinealectomized rats exhibited a significantly higher ratio of reduced to oxidized glutathione at 3-months compared to the sham and melatonin-supplemented rats. This elevation was not found at the 20 -month time-point. Moreover, reduced glutathione levels were significantly higher in the younger compared to the older animals. These results are consistent with the hypothesis that endogenous melatonin has neuroprotective properties, possibly as an antioxidant or potentiation of cellular antioxidant systems. 


\section{ACKNOWLEDGEMENTS}

I would first like to thank my supervisor, Bruce Pappas, for all of the support and guidance that he has given me over the last 7 years. Bruce, it has been an absolute privilege working in your laboratory. You have been an exemplary professor and I have learned many things from you. Through the years I was especially grateful for your friendship and your open-door policy. Whenever I needed to talk (which was often) you always listened. Thank You.

Next, I would like to thank my family. Mom and Dad thank you for all of the support and love that you have given me all of my life. Words cannot express how grateful I am to have you for my parents. I would also like to thank my brother Ray for all of his support through the years. Ray, you and I share the same ambitiousness and strive for excellence which is probably why we get along so well.

Third, I would like to thank Lesley Ritchie for her wonderful gift of friendship. I cannot tell you how grateful I am to have met you. Lesley, thanks for your kindness through the years it meant a lot to me. Working with you in the lab was a lot of fun and we both learned a lot from each other. I look forward to joining you out west!

Next, I would like to thank Kathryn Payne for her friendship in the lab. Thanks for being there for me and listening to me whenever I felt the need to talk. I really appreciated the times when you sat with me while I was counting because as you well know, stereology can be a monotonous task! 
I would also like to thank our animal care staff- Ann Hogarth, Collinda Thivierge, and Andrew Mott- for all of the help you have given me through the years. Your hard work was always appreciated.

A big thanks also goes to Kerry Rennie for her help with the cutting and staining of some of my tissue. I really appreciated the help and if you ever need a letter of reference all you have to do is ask.

Last, but certainly not least, I would like to thank my undergraduate supervisor Dr. Carlyle Smith. Carlyle, if it were not for you and your encouragement, I never would have begun this journey in the first place. 


\section{TABLE OF CONTENTS}

Title Page $\ldots \ldots \ldots \ldots \ldots \ldots \ldots \ldots \ldots \ldots \ldots \ldots \ldots \ldots \ldots \ldots$

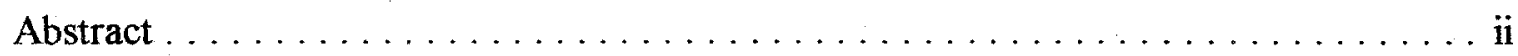

Acknowledgments $\ldots \ldots \ldots \ldots \ldots \ldots \ldots \ldots \ldots \ldots \ldots \ldots \ldots$ iv

Table of Contents $\ldots \ldots \ldots \ldots \ldots \ldots \ldots \ldots \ldots \ldots \ldots \ldots \ldots \ldots$

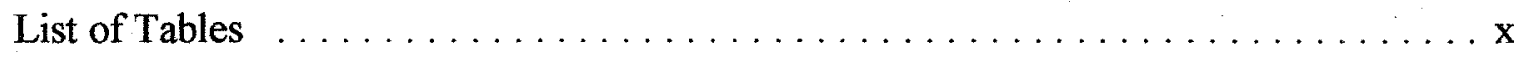

List of Figures $\quad \ldots \ldots \ldots \ldots \ldots \ldots \ldots \ldots \ldots \ldots \ldots \ldots \ldots \ldots \ldots \ldots$

List of Appendices $\ldots \ldots \ldots \ldots \ldots \ldots \ldots \ldots \ldots \ldots \ldots \ldots \ldots \ldots \ldots$

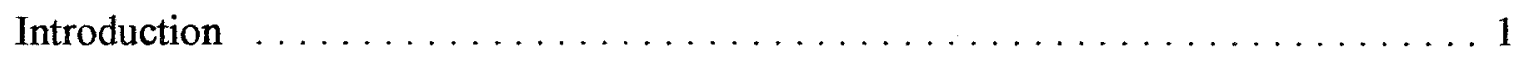

Melatonin Synthesis $\ldots \ldots \ldots \ldots \ldots \ldots \ldots \ldots \ldots \ldots$

Role of Melatonin $\ldots \ldots \ldots \ldots \ldots \ldots \ldots \ldots \ldots \ldots$

Oxygen Free Radicals $\ldots \ldots \ldots \ldots \ldots \ldots \ldots \ldots \ldots$

Superoxide Radical $\ldots \ldots \ldots \ldots \ldots \ldots \ldots \ldots$

Hydrogen Peroxide $\ldots \ldots \ldots \ldots \ldots \ldots \ldots \ldots$

Hydroxyl Radical $\ldots \ldots \ldots \ldots \ldots \ldots \ldots \ldots \ldots \ldots$

Antioxidant Defense System . . . . . . . . . . . . . . . . 9

Antioxidant Actions of Melatonin $\ldots \ldots \ldots \ldots \ldots \ldots \ldots$

Melatonin as a Free Radical Scavenger $\ldots \ldots \ldots \ldots \ldots \ldots$

Antioxidative Properties of Melatonin against DNA damage . . . . . 13

vi 
Melatonin protects against lipid peroxidation in vivo and in vitro 14

Neuroprotective Properties of Melatonin . . . . . . . . . . . . 17

Melatonin protects against kainic acid neurotoxicity $\ldots \ldots \ldots \ldots 17$

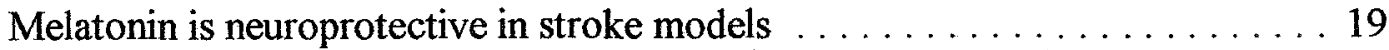

Free radicals, Melatonin, and Neurodegenerative diseases $\ldots \ldots \ldots \ldots 26$

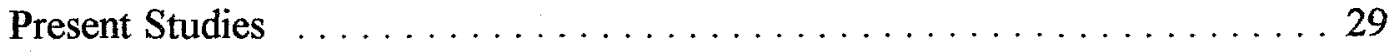

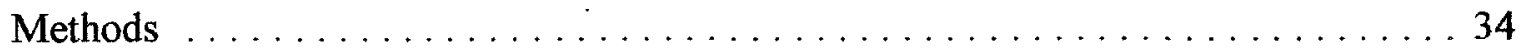

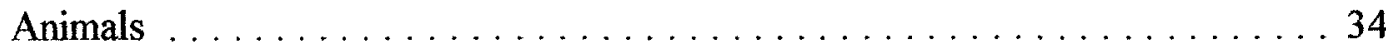

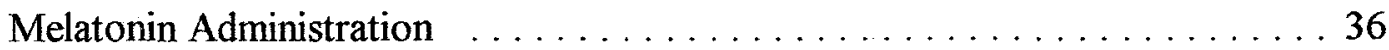

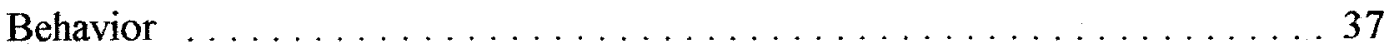

Elevated Plus Maze Testing $\ldots \ldots \ldots \ldots \ldots \ldots \ldots \ldots \ldots$

Morris Water Maze Testing $\ldots \ldots \ldots \ldots \ldots \ldots \ldots$

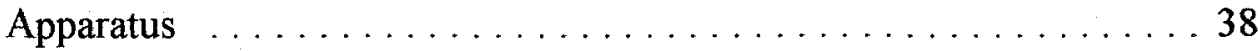

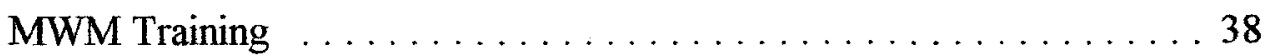

Histological Methods . . . . . . . . . . . . . . . . . . . . . . . . 39

Hippocampal Measures $\ldots \ldots \ldots \ldots \ldots \ldots \ldots \ldots \ldots \ldots$

Biochemical Analyses $\ldots \ldots \ldots \ldots \ldots \ldots \ldots \ldots \ldots \ldots \ldots \ldots$

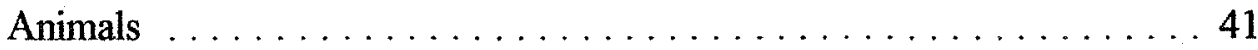

Tissue Collection $\ldots \ldots \ldots \ldots \ldots \ldots \ldots \ldots \ldots \ldots \ldots \ldots$

Thiobarbituric Acid Reactive Substances (TBARS) determination . . . 42

Glutathione (GSH) and Glutathione Disulfide (GSSG) determination . . 43

vii 


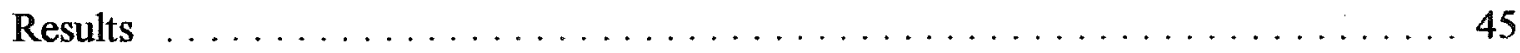

General Health and Weight $\ldots \ldots \ldots \ldots \ldots \ldots \ldots \ldots \ldots \ldots \ldots$

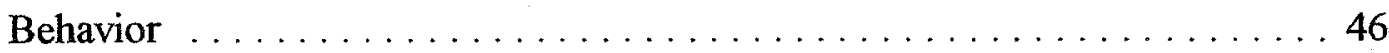

Elevated Plus Maze $\ldots \ldots \ldots \ldots \ldots \ldots \ldots \ldots \ldots \ldots \ldots$

Morris Water Maze $\ldots \ldots \ldots \ldots \ldots \ldots \ldots \ldots \ldots \ldots \ldots \ldots$

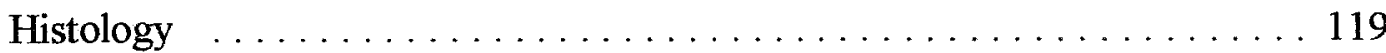

CAl Cell Number $\ldots . \ldots \ldots \ldots \ldots \ldots \ldots \ldots \ldots \ldots . . \ldots \ldots$

CA3 Cell Number $\ldots \ldots \ldots \ldots \ldots \ldots \ldots \ldots \ldots \ldots \ldots \ldots$

Effect of Age on CA1 and CA3 Cell Number ............ 132

Fluoro-Jade B Staining $\ldots \ldots \ldots \ldots \ldots \ldots \ldots \ldots \ldots \ldots$

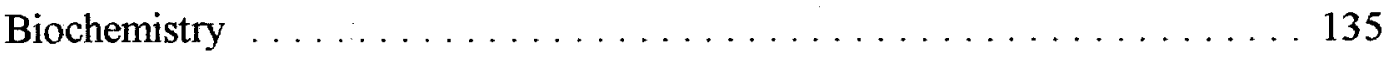

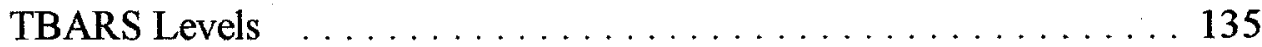

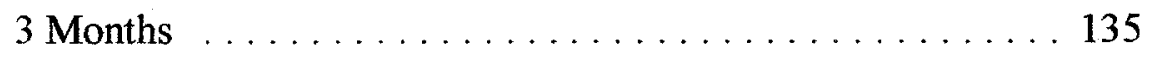

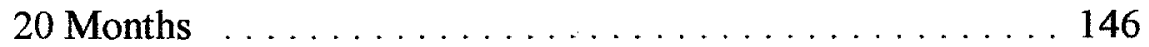

Effect of Age on Hippocampal TBARS Levels $\ldots \ldots \ldots \ldots . .146$

Glutathione Levels ........................ 149

GSH and GSSG Levels at 3-months of age $\ldots \ldots \ldots \ldots \ldots \ldots$

GSH and GSSG Levels at 20-months of age $\ldots \ldots \ldots \ldots \ldots \ldots 151$

Effect of age on GSH and GSSG concentrations $\ldots \ldots \ldots \ldots 156$

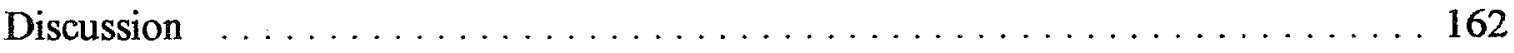

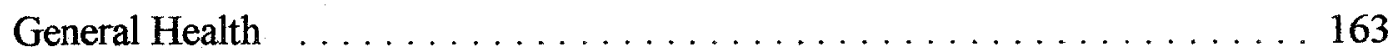

viii 


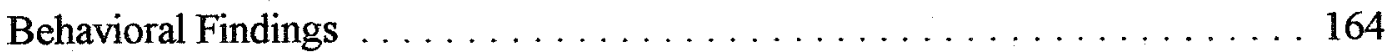

Elevated Plus Maze $\ldots \ldots \ldots \ldots \ldots \ldots \ldots \ldots \ldots \ldots \ldots \ldots$

Morris Water Maze . . . . . . . . . . . . . . . . . . . . . . . . . 169

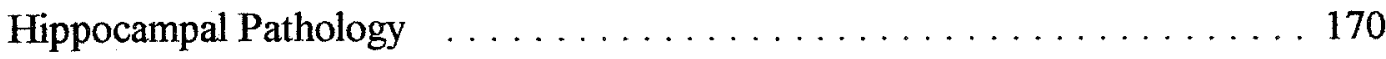

CA1 and CA3 Cell Number $\ldots \ldots \ldots \ldots \ldots \ldots \ldots \ldots \ldots$

Fluoro-Jade B Staining $\ldots \ldots \ldots \ldots \ldots \ldots \ldots \ldots \ldots \ldots \ldots \ldots \ldots \ldots$

TBARS and Glutathione Levels $\ldots \ldots \ldots \ldots \ldots \ldots \ldots \ldots . \ldots \ldots$

Effect of Age on Hippocampal Measures . . . . . . . . . . . 176

Summary and Conclusions $\ldots \ldots \ldots \ldots \ldots \ldots \ldots \ldots \ldots \ldots$

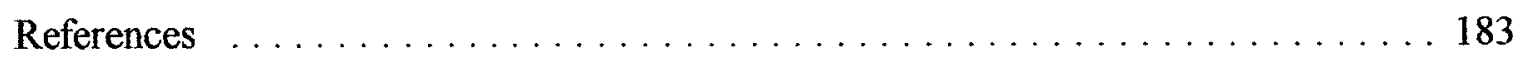

Appendix A . . . . . . . . . . . . . . . . . . . . . . . . . . 194 


\section{LIST OF TABLES}

Table 1. Group weights for sham, melatonin supplemented, and pinealectomized

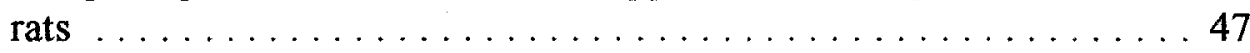

Table 2. TBARS levels in the hippocampus of 3-month old sham, melatonin supplemented, and pinealectomized rats . . . . . . . . . 147

Table 3. TBARS levels in the cortex, hippocampus, and kidney of 20 -month old sham, melatonin supplemented, and pinealectomized rats . . . . . 148

Table 4. Age effects on hippocampal glutathione levels $\ldots \ldots \ldots \ldots \ldots \ldots 1$ 


\section{LIST OF FIGURES}

Figure 1. Open arm entries and ratio of entries into the open arms $\ldots \ldots \ldots 48$

Figure 2. Percentage of time spent on open arms and ratio of protected stretched attend

postures

Figure 3. Percentage of time spent in central hub $\ldots \ldots \ldots \ldots \ldots \ldots$

Figure 4. Total entries and closed arm entries $\ldots \ldots \ldots \ldots \ldots \ldots \ldots \ldots \ldots \ldots$

Figure $5 . \quad$ Number of head dips and number of vertical stretches $\ldots \ldots \ldots 7$

Figure 6. Number of open entries and ratio of open arm entries $\ldots \ldots \ldots 5$

Figure 7. Ratio of time spent on open arms and ratio of protected stretched attend

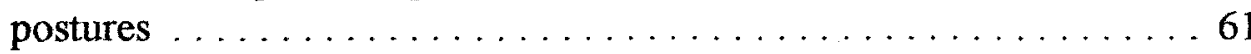

Figure 8. Ratio of time spent in central hub $\ldots \ldots \ldots \ldots \ldots \ldots \ldots \ldots$

Figure 9. Number of total entries and closed arm entries $\ldots \ldots \ldots 6$

Figure 10. Number of head dips and number of vertical stretches $\ldots \ldots \ldots 67$

Figure 11. Number of open entries and ratio of open arm entries $\ldots \ldots \ldots 69$

Figure 12. Ratio of time spent on open arms and ratio of protected stretched attend postures ......................... 71

Figure 13. Ratio of time spent in central hub $\ldots \ldots \ldots \ldots \ldots \ldots \ldots$

Figure 14. Total entries and closed arm entries $\ldots \ldots \ldots \ldots \ldots$

Figure 15. Number of head dips and number of vertical stretches $\ldots \ldots \ldots 78$

Figure 16. Number of open entries and ratio of open entries $\ldots \ldots \ldots \ldots$

Figure 17. Ratio of time spent on open arms and ratio of protected stretched attend postures . . . . . . . . . . . . . . . . . . 82

Figure 18. Ratio of time spent in central hub $\ldots \ldots \ldots \ldots \ldots$

xi 
Figure 19. Total entries and closed arm entries $\ldots \ldots \ldots \ldots \ldots \ldots$

Figure 20. Head dips and vertical stretches $\ldots \ldots \ldots \ldots \ldots \ldots \ldots$

Figure 21. Number of open entries and ratio of open entries $\ldots \ldots \ldots \ldots \ldots 91$

Figure 22. Ratio of time spent on open arms and ratio of protected stretched attend postures ............................. 93

Figure 23. Ratio of time spent in central hub $\ldots \ldots \ldots \ldots \ldots \ldots \ldots \ldots$

Figure 24. Total entries and number of closed entries $\ldots \ldots \ldots \ldots \ldots . . . . .99$

Figure $25 . \quad$ Head dips and vertical stretches $\ldots \ldots \ldots \ldots \ldots \ldots \ldots \ldots 10 \ldots \ldots$

Figure 26. Hidden platform water maze $\ldots \ldots \ldots \ldots \ldots \ldots \ldots \ldots \ldots . \ldots \ldots$

Figure 27. Hidden platform water maze $\ldots \ldots \ldots \ldots \ldots \ldots \ldots \ldots \ldots$

Figure 28. Hidden platform water maze $\ldots \ldots \ldots \ldots \ldots \ldots \ldots . \ldots . \ldots$

Figure 29. Hidden platform water maze $\ldots \ldots \ldots \ldots \ldots \ldots \ldots \ldots \ldots \ldots$

Figure 30. Hidden platform water maze $\ldots \ldots \ldots \ldots \ldots \ldots \ldots \ldots \ldots \ldots \ldots$

Figure 31 Hidden platform water maze at 11 -months post-surgery $\ldots \ldots \ldots 115$

Figure 32. Age effects on platform location latency $\ldots \ldots \ldots \ldots \ldots \ldots \ldots$

Figure 33. Age effects on platform location path-length $\ldots \ldots \ldots \ldots \ldots \ldots$

Figure 34. Estimated hippocampal CAl cell counts $\ldots \ldots \ldots \ldots \ldots \ldots \ldots$

Figure 35. Photomicrograph of CA1 pyramidal layer from a sham, melatonin supplemented, and pinealectomized rat $\ldots \ldots \ldots \ldots \ldots \ldots . \ldots \ldots$

Figure 36. Estimated hippocampal CA3 cell counts $\ldots \ldots \ldots \ldots \ldots \ldots 128$

Figure 37. Photomicrograph of CA3 pyramidal cell layer from a sham, melatonin 130

Figure 38. Hippocampal CA1 Fluoro-Jade B cell counts $\ldots \ldots \ldots \ldots \ldots \ldots$

Figure 39. Hippocampal CA3 Fluoro-Jade B cell counts $\ldots \ldots \ldots \ldots \ldots \ldots$

xii 
Figure $40 . \quad$ Hippocampal CA4 Fluoro-Jade B cell counts . . . . . . . . 140

Figure 41. Fluoro-Jade B cell counts in dentate gyrus $\ldots \ldots \ldots \ldots \ldots \ldots$

Figure 42. Hippocampal Fluoro-Jade B cell counts $\ldots \ldots \ldots \ldots \ldots \ldots \ldots$

Figure 43. Age effects on hippocampal TBARS levels $\ldots \ldots \ldots \ldots \ldots \ldots$

Figure 44. Ratio of GSH to GSSG at 3-months post-surgery $\ldots \ldots \ldots \ldots \ldots$

Figure 45. GSH and GSSG levels at 3 -months post-surgery $\ldots \ldots \ldots \ldots$

Figure 46. GSH and GSSG levels at 20 -months post-surgery $\ldots \ldots \ldots \ldots$

Figure 47. Ratio of GSH to GSSG at 20-months post-surgery $\ldots \ldots \ldots \ldots$ 


\section{LIST OF APPENDICES}

A. Appendix of ANOVA Summary Tables . . . . . . . . . . . . . . 194

A.1. ANOVA Summary Tables for body weight $\ldots \ldots \ldots \ldots \ldots \ldots \ldots$

A.2. ANOVA Summary Tables for elevated plus maze ... . . . . . . 195

A.3. ANOVA Summary Tables for Morris water maze $\ldots \ldots \ldots \ldots \ldots \ldots 212$

A.4. ANOVA Summary Tables for hippocampal cell counts $\ldots \ldots \ldots \ldots \ldots \ldots$

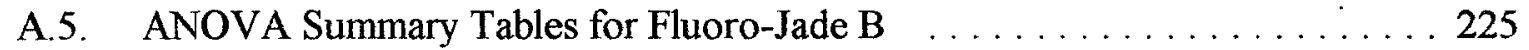

A.6. ANOVA Summary Tables for TBARS levels $\ldots \ldots \ldots \ldots \ldots \ldots \ldots$

A.7. ANOVA Summary Tables for glutathione levels $\ldots \ldots \ldots \ldots \ldots \ldots$ 


\section{LIST OF ABBREVIATIONS}

$A \beta$ - amyloid beta peptide

BRDU- 5-bromo-2-deoxyuridine

CAT- catalase

CerAo- reversible cerebral artery occlusion

DMPO-5,5-dimethylpyraline-N-oxide

GPx- glutathione peroxidase

GSH- glutathione

GSSG- oxidized glutathione

4-HDA- 4-hydroxyalkenals

MCAO- middle cerebral artery occlusion

MDA- malonaldehyde

MEL X PIN- melatonin supplemented pinealectomized rats

MPO- myeloperoxidase

MPTP-1-methyl-4-phenyl-1,2,3,6- tetrahydropyridine

MRI- magnetic resonance imaging

MWM- morris water maze

8-OHDG- 8-hydroxy-2-deoxyguanosine

8-oxodGuo- 8,oxo-7,8-dihydro-2' deoxyguanosine

PIN- pinealectomized

RAM- radial arm maze

SCN- suprachiasmatic nucleus

SOD- superoxide dismutase

TBARS- thiobarbituric acid reactive substances

2VO- permanent common carotid artery occlusion 
The pineal gland is the principal source of circulating melatonin (N-acetyl-5methoxytryptamine). In mammals, melatonin is involved in a wide range of physiological processes such as controlling seasonal rhythms and seasonal reproduction (Reiter, 1991). More recently, melatonin has been found to be a potent antioxidant and free radical scavenger and as a result, to have seemingly potent neuroprotective properties when administered both in vitro and in vivo. These findings suggest that pinealectomy which lowers brain melatonin levels (Pang, 1982), may result in an increased vulnerability of neurons to the accumulation of free radical products, particularly that which occurs with advancing age (Reiter, 1991).

The purpose of the present study was to systematically examine the time course of the effects of pinealectomy, over a substantial portion of the life span, on hippocampal pyramidal cell integrity and on behavior. It was also determined if melatonin supplementation could attenuate the neuropathological and behavioral effects (if any) at systematic intervals after pinealectomy. Thirdly, free radical activity was assessed using indices of lipid peroxidation and endogenous antioxidant function in short-term and longterm pinealectomized animals.

\section{Melatonin Synthesis}

The pineal gland located at the top of the midbrain, synthesizes and secretes the hormone melatonin ( $\mathrm{N}$-acetyl-methoxytryptamine). The pineal gland is quite small weighing approximately $120 \mathrm{mg}$ in humans and only $1 \mathrm{mg}$ in the rat. Although located within the brain, the pineal gland is not actually part of the brain because the blood brain barrier physically separates it from the brain. The pineal gland consists of two main types 
of cells: pinealocytes and neuroglial cells. The most prevalent cells in the pineal gland are the pinealocytes, which produce melatonin and peptides such as arginine vasotocin (Brzezinski, 1997).

The functioning of the pineal gland and the production of melatonin, is controlled by the photoperiodic environment (Reiter, 1991). The daily rhythm of melatonin production by the pineal gland is produced by an endogenous circadian clock in the suprachiasmatic nuclei (SCN) of the hypothamus and is communicated to the pineal gland via an intricate pathway (Perreau-Lenz et al., 2003). Firstly, information regarding the photoperiod is transmitted from the retina to the suprachiasmatic nucleus of the hypothalamus via the retinohypothalamic tract (Cavallo, 1993; Perreau-Lenz et al., 2003). This information is then transmitted to the superior cervical ganglion via a multisynaptic pathway that includes the paraventricular nucleus, medial forebrain bundle, and the mediolateral nuclei of the upper thoracic spinal cord. During the dark period (nighttime), noradrenergic sympathetic neurons of the superior cervical ganglion that impinge upon the pinealocytes, release norepinephrine to stimulate melatonin synthesis and release (Cagnacci, 1996).

Melatonin is derived from the amino acid tryptophan. During the light period, the catalyst tryptophan hydroxylase converts tryptophan to 5-hydroxytryptophan (Sugden, 1989). Aromatic L-amino acid decarboxylase then converts 5-hydroxytryptophan to 5hyroxytryptamine (serotonin). During the daily night period, $\mathrm{N}$-acetyltransferase an enzyme found in the pineal gland, converts serotonin to $\mathrm{N}$-acetylserotonin with the aid of acetyl-coenzyme A. Melatonin is then produced by the action of the pineal enzyme hydroxyindole-O-methyl transferase on $\mathrm{N}$-acetylserotonin. 
Apart from the pineal gland, the retina secretes melatonin locally. The retinal photoreceptor utilizes a similar biosynthetic pathway as the pineal gland for converting serotonin to melatonin. Retinal melatonin is thought to be involved in controlling cyclic day-night changes in photoreceptor and retinal pigment epithelium function as well as controlling the sensitivity of the horizontal cells to light (Vanecek, 1998).

During the daily dark period, melatonin is secreted from the pineal gland either directly into the bloodstream or into the cerebrospinal fluid prior to entering the bloodstream. Once released by the pineal gland, melatonin is capable of entering all cells of the body because it is both lipid and water-soluble (Reiter, 1996). Moreover, this indole readily crosses the blood brain barrier which enables it to exert its various functions on cells located anywhere in the organism, including the brain. Some cellular effects of melatonin are mediated by high-affinity melatonin receptors of which there are two major subtypes. Both the $\mathrm{mel}_{1}$ and $\mathrm{mel}_{2}$ subtypes belong to the seven transmembrane $\mathrm{G}$ protein-coupled receptors superfamily and are expressed in various brain regions such as the pars tuberalis of the pituitary, suprachiasmatic nucleus, medial preoptic area, anterior hypothalamus, dorsomedial and ventromedial hypothalamic nuclei, paraventricular nucleus, hippocampus, cerebral and cerebellar cortex area (Vanecek, 1998). In the retina, the effects of melatonin are mediated by the mel $_{2}$ receptor.

\section{Role of Melatonin}

Melatonin was first described as an indole with hormonal and chronobiological functions (Reiter, 1991). The suprachiasmatic nucleus is important in the control of circadian rhythms (Armstrong, 1989). As previously mentioned, melatonin binding sites 
have been found in the suprachiasmatic nucleus and seem to be important sites of melatonin's circadian effects (Reiter, 1991). Melatonin is believed to affect the circadian and seasonal timing of various physiological and behavioral processes in mammals such as sexual maturation and seasonal reproduction (Ebling \& Foster, 1989). For many mammalian species, sexual maturation and the ability to reproduce, occurs at a time that ensures that future offspring are born at the most ideal time of the year for that species (Ebling \& Foster, 1989). The duration of melatonin secretion during the dark period changes across the seasons and provides mammals information regarding day-length. For example, duration of melatonin secretion is short in the short nights of summer and longer in the long nights of winter. The nightly secretion of melatonin provides many species with an internal hormonal representation of the length of night that reflects the season of the year.

Melatonin binding sites involved in mediating the reproductive effects of melatonin are located in the pars tuberalis of the pituitary and the medial basal hypothalamus (Reiter, 1991). Melatonin acts on the pars tuberalis of the pituitary to regulate prolactin secretion and the medial basal hypothalamus to regulate gonadropins.

In addition to its chronobiological functions, melatonin has also been suggested to have many psychopharmacological effects such as hypnotic, analgesia, and anxiolytic properties (Kopp et al., 1999). The sleep-inducing effects of melatonin have been clearly documented. The administration of melatonin prior to sleep onset has been found to significantly improve sleep (Haimov et al., 1995). Haimov and colleagues (1995) found that the administration of melatonin 2 hours before sleep onset to elderly individuals with insomnia significantly reduced the amount of time it took to fall asleep. Similar effects 
have also been observed when melatonin was administered in normal adult individuals with doses ranging from $0.3 \mathrm{mg}$ to $1 \mathrm{mg}$ (Zhadanova et al., 1995).

In addition, in many species including humans, melatonin has been found to have analgesic activities. Several studies using rodents have found that the administration of melatonin at doses that range from $20-200 \mathrm{mg} / \mathrm{kg}$ produced a significant analgesic effect following various classical tests such as tail flick or hot plate (Ebadi et al., 1998;

Golombek et al. 1991; Golombek et al., 1996; Lakin et al., 1981).

A number of studies have also found anxiolytic effects of melatonin (Golombek et al., 1996; Kato et al., 1998; Neville et al., 1986). For instance, melatonin has been found to protect against stress-induced gastric lesions in rats (Kato et al., 1998) as well as the effects of chronic stress (Brotto et al., 2001; Kopp et al., 1999). Significantly, melatonin has also been found to augment the well-known anti-anxiety effects of benzodiazepines in various test models for anxiolytics in mice such as the elevated plus maze task (Golombek et al., 1993; Guardiola et al., 1992). In addition to melatonin's chronobiological, sedative, anxiolytic, and analgesic functions, this indole has also been shown to be a potent antioxidant and to have powerful free radical scavenging properties (Tan et al., 1993).

\section{Oxygen Free Radicals}

Oxygen-derived free radicals are continuously produced within cells of aerobic organisms specifically because they use molecular oxygen (dioxygen or $\mathrm{O}_{2}$ ) as the basis of their metabolism. Free radicals are molecules or atoms that contain an unpaired electron in their outermost orbital. This renders them highly reactive and if they interact 
with non-radical molecules, they can be highly damaging. Because free radicals are unstable, they are capable of donating or taking an electron from nearby organic and inorganic molecules in order to achieve stability. The most notable reactive species derived from $\mathrm{O}_{2}$ are the superoxide and the hydroxyl radical.

\section{Superoxide Radical}

The addition of a single electron to molecular oxygen yields the superoxide radical, $\mathrm{O}_{2}^{-}$(Eq.1). The superoxide radical is generated in a variety of ways one of which is through the actions of electron transport chains in mitochondria. Often, electrons can escape through the electron transport chain where they can react with molecular oxygen to form the superoxide radical.

$$
\mathrm{O}_{2}+1 \text { electron } \Rightarrow \mathrm{O}_{2}(\text { (Eq.1) }
$$

The superoxide radical can also be formed when phagocytic cells such as neutrophils, and macrophages are activated in response to infectious organisms (Halliwell, 1992). It has been suggested that formation of the superoxide anion may provide an additional defense against infectious organisms (Halliwell, 1992). Hence, the controlled production of the superoxide radical can be beneficial in some circumstances. In excessive amounts however, the superoxide radical can lead to damaging effects and it is believed that this radical is the primary mediator of oxygen toxicity (Fridovich, 1983).

The primary endogenous defense against superoxide toxicity is the superoxide dismutases, which are enzymes that catalytically scavenge the superoxide radical. There 
are two different types of superoxide dismutases. The first is a copper-zinc superoxide dismutase that is primarily found in the cytosol of eukaryotic cells while the second is a manganese superoxide dismutase found in mitochondria (Fridovich, 1983).

The dismutation of the superoxide radical by superoxide dismutase, results in the formation of hydrogen peroxide and oxygen (Eq. 2).

$$
\mathrm{O}_{2}+\mathrm{O}_{2}+2 \mathrm{H} \rightarrow \mathrm{H}_{2} \mathrm{O}_{2}+\mathrm{O}_{2}(\mathrm{Eq} \cdot 2)
$$

\section{Hydrogen Peroxide}

Hydrogen peroxide is a potent oxidizing agent but is not defined as a free radical because it does not contain any unpaired electrons (Halliwell \& Gutteridge, 1984). Superoxide dismutase is not the only enzyme that generates hydrogen peroxide. Other enzymes such as monoamine oxidase and zanthene oxidase are also capable of generating hydrogen peroxide (Cohen, 1984). Monoamine oxidase is responsible for metabolizing catecholamines such as dopamine and norepinephine. Hydrogen peroxide is a product of these reactions (Cohen, 1984).

Two enzymes, catalase and glutathione peroxidase function as protective mechanisms against hydrogen peroxide. Catalase removes hydrogen peroxide by converting it to water and oxygen. Catalase is primarily found in peroxisomes, lysosomes, and mitochondria. Hence, it is not as efficacious in affording protection against oxyradical injury to cytosolic and nuclear components. Glutathione peroxidase is found in mitochondria and cytoplasm. Glutathione peroxidase affords protection because it eliminates hydrogen peroxidase and lipid peroxides by reduction utilizing glutathione. 
Hydrogen peroxide itself is not highly toxic to various tissues. However it is capable of reacting with metal ions such as iron and copper to form highly oxidizing tissue damaging radicals (Reiter, 1995).

\section{Hydroxyl Radical}

When hydrogen peroxide comes into contact with iron or copper, the highly toxic hydroxyl radical is formed by the Fenton reaction (Eq. 3). Hydroxyl radicals are also generated when metal ions react with the superoxide radical in what is known as the Haber-Weiss reaction (Cohen, 1984) (Eq. 4). The hydroxyl radical is detrimental to proteins essential for membrane function as well as membrane-bound enzymes and receptors (Halliwell, 1992).

$$
\begin{gathered}
\mathrm{Fe}^{2+}+\mathrm{H}_{2} \mathrm{O}_{2} \rightarrow \mathrm{Fe}^{3+}+\mathrm{OH}+\mathrm{OH}_{(\mathrm{Eq} \cdot 3)} \\
\mathrm{O}_{2}+\mathrm{H}_{2} \mathrm{O}_{2}+\mathrm{Fe} \text { catalyst } \rightarrow \mathrm{OH}+\mathrm{OH}+\mathrm{O}_{2(\mathrm{Eq} \cdot 4)}
\end{gathered}
$$

The hydroxyl radical is also capable of instigating the free radical chain reaction known as lipid peroxidation. During the process of lipid peroxidation, a free radical such as the superoxide or hydroxyl radical reacts with a polyunsaturated fatty acid chain such as arachidonic acid, and generates a carbon-centered radical (lipid ) in the lipid membrane (Halliwell, 1992) (Eq. 5). This lipid radical can then react with molecular oxygen to generate the peroxyl and alkoxyl radicals (Eq. 6).

$$
\text { Lipid-H + radical } \rightarrow \text { lipid }+ \text { radical-H }(\text { (Eq. 5) }
$$




$$
\text { lipid }+\mathrm{O}_{2} \rightarrow \text { lipid- } \mathrm{O}_{2}{ }_{(\mathrm{Eq} .6)}
$$

If this free radical chain reaction is not terminated by an antioxidant, it will continue to damage lipid membranes and can abstract hydrogen atoms from adjacent fatty acid side chains (Eq. 7). The consequence of this damage is that integral proteins will be affected resulting in alterations in both enzyme and receptor function (Halliwell, 1992).

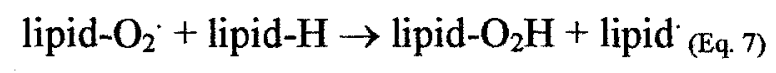

The brain is particularly vulnerable to free radical damage for several reasons. First, its membrane lipids are rich in polyunsaturated fatty acids that are quite vulnerable to free radical insult (Lohr, 1991). Second, the brain's levels of various antioxidant enzymes such as catalase and glutathione peroxidase are low hence making it more vulnerable to free radical attack (Halliwell, 1992). Third, a number of brain regions such as the substantia nigra and globus pallidus, contain high iron content. This can be detrimental when there are concomitantly high levels of hydogen peroxide as this situation will initiate the formation of the toxic hydroxyl radical.

\section{Antioxidant Defense System}

Free radical production is a normal consequence of aerobic metabolism. As a protective mechanism, all organisms have antioxidant defense systems that either restrict the generation of free radicals or render them ineffective when they are generated (Reiter, 1996). The antioxidant defense system is comprised of the enzyme superoxide dismutase 
(SOD), glutathione peroxidase (GPx), and catalase (CAT). As previously mentioned, superoxide dismutase scavenges the superoxide radical while gluathione peroxidase and catalase remove hydrogen peroxide (Fridovich, 1983).

These enzymes function together to convert potentially harmful radicals into inactive substances. For instance, the excessive production of the superoxide radical requires a compensatory increase in superoxide dismutase levels in order to inactivate this radical. The dismutation of the superoxide radical by superoxide dismutase, results in the production of hydrogen peroxide. Thus, if levels of superoxide dismutase are high, so can be the level of hydrogen peroxide. Hence, a compensatory increase in either catalase or glutathione peroxidase is also required. If levels of catalase or glutathione peroxidase are low, the hydrogen peroxide generated by the dismutation of the superoxide radical may result in the formation of hydroxyl radicals producing an escalation in radicalinduced tissue damage (Lohr, 1991). The escalation of free radical production places a great demand on the antioxidant defense system to limit their damaging reactions. This increased demand on antioxidant mechanisms is termed oxidative stress.

\section{Antioxidant Actions of Melatonin}

\section{Melatonin as a free radical scavenger}

In addition to melatonin's role in mammalian chronobiological functions, this indole has also been shown to be a potent antioxidant (Tan et al., 1993). Several studies have provided evidence for its role as a free radical scavenger (Marshall et al., 1996; Pieri et al., 1994). For instance, Tan and colleagues (1993) created hydroxyl radicals ( $\mathrm{OH})$ by exposing hydrogen peroxide $\left(\mathrm{H}_{2} \mathrm{O}_{2}\right)$ to ultraviolet light (UV). Because the $\mathrm{OH}$ radical is 
short-lived, these investigators utilized a spin-trapping reagent; 5,5-dimethylpyraline- $\mathrm{N}$ oxide (DMPO) that produced OH-DMPO adducts. The spin adducts were detected by high-performance liquid chromatography with electron spin resonance.

Tan et al. (1993) found that melatonin was a more effective hydroxyl radical scavenger than either glutathione (GSH) or mannitol. Only $21 u \mathrm{M}$ of melatonin was needed in order to neutralize $50 \%$ of the radicals produced whereas $123 u \mathrm{M}$ of GSH and $283 u \mathrm{M}$ of mannitol was required. The finding that melatonin was a potent scavenger of the $\mathrm{OH}$ radical was an important one because this radical is thought to be the prime mediator of oxygen toxicity, capable of damaging proteins, carbohydrates, DNA, and lipids (Kehrer, 1993).

Following the finding that melatonin was a scavenger of the toxic $\mathrm{OH}$ radical, Pieri et al. (1994) examined the ability of melatonin to scavenge the peroxyl radical (ROO) The scavenging ability of melatonin was compared to that of the well known dietary antioxidants, vitamin $\mathrm{C}$ and $\mathrm{E}$, as well as glutathione. Utilizing an in vitro system, Pieri and colleagues (1994) found that melatonin was twice as effective in neutralizing the toxic peroxyl radicals than vitamin $\mathrm{E}$ and $\mathrm{C}$. Moreover, melatonin was three times as effective in scavenging the ROO radical than glutathione.

In addition to its role as a free radical scavenger, melatonin has also been shown to influence the activity of the antioxidative enzymes glutathione peroxidase, superoxide dismutases, and catalase (Baydas et al., 2002; Okatani et al., 1995; Rodriguez et al., 2004). Barlow-Walden and colleagues (1995) were one of the first groups to investigate the effects of melatonin on brain glutathione peroxidase activity. Rats were administered intraperitoneal injections of melatonin $(500 \mathrm{ug} / \mathrm{kg})$ during the day and then sacrificed 
either 30 or 180 minutes following melatonin treatment. These investigators found that brain glutathione peroxidase activity was increased 2-fold 30 minutes following melatonin treatment. This increase was still evident in the brains of rats sacrificed 180 minutes following the administration of melatonin.

The lowering of endogenous melatonin by pinealectomy (surgical removal of the pineal gland), has been shown to alter glutathione peroxidase activity. Baydas et al. (2002) examined both glutathione peroxidase and oxidized glutathione (GSSG) activity, and lipid peroxidation in the brain and kidney of pinealectomized animals. One month following pinealectomy, animals were sacrificed and tissue samples of brain, liver, and kidney were collected. Baydas and colleagues found that glutathione peroxidase activity was lowered in the pinealectomized animals. Oxidized glutathione activity in brain and liver tissues was also lowered among the pinealectomized animals. In addition, lipid peroxidation was increased in the brain and kidney among the pinealectomized compared to the control animals. In summary, these investigators found that melatonin deficiency caused by pinealectomy resulted in reduced glutathione peroxidase activity levels in several tissues. Lowered glutathione peroxidase activity was also associated with higher lipid peroxide products in these tissues.

Melatonin has also been shown to influence antioxidant gene expression. Mayo et al. (2002) examined the effect of melatonin on antioxidant gene expression in different neuronal cells lines. Mayo et al. found that melatonin at physiological serum concentrations ( $1 \mathrm{nM})$ increased the mRNA of both superoxide dismutases and gluthione peroxidase in two neuronal cell lines. 
Antioxidative properties of melatonin against DNA damage

Following their finding that melatonin was an effective free radical scavenger; Tan and colleagues (1993) investigated melatonin's antioxidant properties in vivo. Nuclear DNA is highly vulnerable to damage caused by oxygen free radicals. Thus, Tan et al. $(1993,1994)$ conducted two studies to determine whether melatonin was capable of protecting hepatic nuclear DNA from damage inflicted by free radicals generated by the carcinogen safrole. These investigators found extensive DNA adducts in the hepatic tissue of rats that were administered $300 \mathrm{mg} / \mathrm{kg}$ of safrole. Administration of melatonin at concentrations of $0.2 \mathrm{mg} / \mathrm{kg}$ and $0.4 \mathrm{mg} / \mathrm{kg}$ dose-dependently reduced DNA-adduct formation (Tan et al., 1993). These results demonstrated that melatonin afforded protection against DNA damage caused by safrole.

In a follow-up study, Tan et al. (1994) examined the effectiveness of physiological concentrations of melatonin in protecting hepatic DNA from damage inflicted by safrole. Rats were administered safrole at a dosage of $100 \mathrm{mg} / \mathrm{kg}$ either during the day, when melatonin levels are at their lowest, or at night when melatonin levels are high. Tan and colleagues (1994) found that rats who had received the safrole at night exhibited less DNA damage than rats that had received the safrole during the day. Thus, the nighttime rise in melatonin levels was enough to reduce the damage due to the carcinogen safrole.

In addition, Tan et al (1994) found that rats that were subjected to pinealectomy exhibited the hightest DNA damage following the administration of safrole. DNA damage was lessened in the pinealectomized animals when they were given exogenous 
melatonin. Collectively, these results suggested that even at physiological levels, melatonin is an effective antioxidant in vivo.

In a more recent study, Karbownik et al. (2001) examined the effect of melatonin on estradiol-induced oxidative damage to DNA and cellular membranes in hamster kidney, liver, and testes. Hamsters were administered estradiol ( $75 \mathrm{mg} / \mathrm{kg}$ body wt i.p.) that has pro-oxidative effects on DNA. Other animals were also given melatonin (15 $\mathrm{mg} / \mathrm{kg}$ body wt. i.p.) 30 minutes prior to estradiol treatment and 120 minutes following estradiol treatment. Three or five hours following estradiol administration, measurement of 8-oxo-7,8-dihydro-2 deoxyguanosine (8 oxodGuo) levels an indicator of DNA damage, and MDA and 4-HDA levels, an index of lipid peroxidation, were collected in kidneys, liver, and testes.

Karbownik and colleagues found that estradiol treatment resulted in an increase in 8-oxodGuo levels in both the kidney and liver of animals. Administration of melatonin prevented the estradiol induced DNA damage in both organs. Moreover, melatonin produced a reduction in lipid peroxidation products further supporting that this indole has antioxidative actions in reducing oxidative damage to both DNA and to cellular membranes.

\section{Melatonin protects against lipid peroxidation in vivo and in vitro}

In addition to its ability to protect against DNA damage, melatonin has also been found to protect against lipid peroxidation in vivo (Melchiorri et al., 1995). Melchiorri et al. (1995) treated rats with paraquat, a herbicide that causes damage to the lungs, liver, and kidneys of both animals and humans. Lipid peroxidation is believed to occur in 
paraquat toxicity. Thus, Melchiorri and colleagues (1995) examined the effectiveness of melatonin against paraquat-induced oxidative damage in rat lung and liver. Two indices, concentration of malonaldehyde (MDA) and 4-hydroxyalkenals were used in order to measure lipid peroxidation. These investigators found that rats treated with paraquat at a concentration of 20 or $70 \mathrm{mg} / \mathrm{kg}$ exhibited elevated tissue levels of both MDA and 4hydroxyalkenals. These investigators also administered $10 \mathrm{mg} / \mathrm{kg}$ of melatonin to rats prior to the administration of paraquat and every 6 hours thereafter, and found that melatonin eliminated the rise in MDA and 4-hydroxyalkenal levels seen in paraquat treated rats. These results demonstrated that melatonin was effective in protecting against lung and liver oxidative damage inflicted by paraquat.

More recently, Carneiro and Reiter (1998) examined both the in vivo and in vitro effect of melatonin on aminolevulinic-induced lipid peroxidation in rat cortex, cerebellum, and hippocampus. An increase in aminolevulinic acid is found during acute intermittent porphyria and results in neuropsychiatric manifestations such as epileptictype seizures and motor neuropathy.

Concentrations of MDA and 4-HDA were used in order to assess aminolevulinic acid induced membrane oxidative damage. Rats were randomly assigned to one of three groups: saline-treated controls, aminolevulinic acid treated rats and aminolevulinic acid treated animals that also received melatonin. Control animals were injected with saline (0.9\% i.p.) three times a day at 6 -hour intervals. Aminolevulinic acid treated rats received aminolevulinic injections $(40 \mathrm{mg} / \mathrm{kg}$ ) every other day. Following the injection, rats received two saline injections at 6-hour intervals. On the days that they did not receive aminolevulinic acid, rats were given saline injections three times a day at 6-hour 
intervals. Rats in the aminolevulinic acid/melatonin group were given melatonin (10 $\mathrm{mg} / \mathrm{kg}$ ) injections 30 minutes prior to aminolevulinic treatment. Following the aminolevulinic acid injection, melatonin was injected twice at 6-hour intervals. All treatments continued for seven days.

Carneiro and Reiter (1998) found that rats treated with aminolevulinic acid had elevated MDA and 4-hyroxyalkenal levels in the cerebellum and hippocampus but not in cortex. By contrast, rats treated with both aminolevulinic and melatonin exhibited MDA and 4-hydroxyalkenal levels comparable to control rats. These results demonstrated that melatonin afforded protection against aminolevulinic acid induced oxidative damage.

In the same report, Carneiro and Reiter (1998) also examined melatonin's effectiveness to protect against aminolevulinic acid induced lipid peroxidation in vitro. In this study, brain homogenates were taken from cortex, hippocampus, and cerebellum. Aminolevulinic acid ( $3 \mathrm{mM})$ was added to cortical, cerebellar, and hippocampal homogenates for 1-4 hours. These investigators found increased levels of MDA and 4hydroxyalkenals due to the administration of aminolevulinic acid in cerebellar, cortical, and hippocampal homogenates. This elevation of lipid peroxidation products was both concentration-dependent and time-dependent.

In addition, vitamin $\mathrm{E}(0.5,1.0$, or $4.0 \mathrm{mM})$ or melatonin $(0.1,0.5,1.0,3.0$, or 4.0 $\mathrm{mM}$ ) was administered in conjunction with aminolevulinic acid in order to examine their antioxidative properties in vitro. It was observed that both melatonin and vitamin $\mathrm{E}$ dosedependently protected against lipid peroxidation (Carneiro \& Reiter, 1998). 


\section{Neuroprotective Properties of Melatonin}

\section{Melatonin Protects against Kainic Acid Neurotoxicity}

Other evidence supporting the neuroprotective effect of melatonin has come from studies utilizing the excitotoxin, kainic acid (Dykens et al., 1987; Melchiorri et al., 1995). Kainic acid is a non-degradable analog of glutamate that is both neuroexcitatory and neurotoxic. Administration of kainic acid to rodents produces brain damage comparable to that seen in a number of neurodegenerative conditions such as Huntington's disease and reperfusion tissue injury (Dykens et al., 1987). The tissue injury observed after the administration of kainic acid is believed to involve free radicals.

Several studies have examined whether melatonin affords protection against kainate-induced toxicity. For instance, Melchiorri et al. (1995) examined whether melatonin protected against kainate-induced toxicity in brain homogenates. Tissue concentrations of MDA and 4-hydroxyalkenals were used as indices of lipid peroxidation. Brain homogenates from cerebral cortex, cerebellum, hippocampus, hypothalamus, and corpus striatum were exposed to kainic acid $(11.7 \mathrm{mM})$ for 20 minutes. Kainic acid treatment resulted in an elevation in lipid peroxidation products. By contrast, brain homogenates incubated with kainic acid combined with melatonin (from 0.1 to $4 \mathrm{mM}$ ) showed reduced accumulations of lipid peroxidation products in a dosedependent manner.

In additon to finding that melatonin was protective against kainic acid treatment in vitro, investigators have also shown that it is protective in vivo. For instance, Guisti and colleagues (1996) injected rats with kainic acid (10 mg/kg i.p.) alone, or combined with melatonin $(2.5 \mathrm{mg} / \mathrm{kg})$. Rats were given melatonin 20 minutes prior to kainic acid 
treatment, immediately following, and 1 and 2 hours following the administration of kainic acid. Rats treated with kainic acid exhibited neurobehavioral changes such as head nodding, wet dog shakes and masticatory movements. Moreover, they exhibited marked neuronal damage in the hippocampus, pyriform cortex, and amygdala. Biochemical changes, such as a decrease in norepinephrine and an elevation in 5-hydroxy-indoleacetic acid were also observed.

The administration of melatonin prevented the neurobehavioral changes seen in rats treated with kainic acid. Moreover, no hippocampal cell loss was observed in the melatonin-kainic acid treated rats. In addition, Guisti et al. did not find any decreases in noradrenaline or increases in 5-hydroxy-indoleacetic acid in melatonin treated animals. Based on these findings, these investigators concluded that melatonin, due to its antioxidative properties, affords protection against kainic-induced brain damage.

In a similar study, Manev and colleagues (1996) administered intraperitoneal injections of kainate to pinealectomized or control rats at a dosage of $10 \mathrm{mg} / \mathrm{kg}$. Following the administration of kainic acid, both the pinealectomized and shampinealectomized rats experienced wet-dog shakes, chronic convulsions, and tonic-clonic convulsions. There were no differences in the severity or duration of the seizures between the pinealectomized and sham-pinealectomized animals. These investigators found neuronal damage in the hippocampus, entorhinal cortex, and amygdala in both the pinealectomized and sham-pinealectomized rats. However, the damage was more extensive in the pinealectomized rats.

More recently, Ortiz and colleagues (2001) examined the neuroprotective effects of melatonin, vitamin $\mathrm{E}$, and estrogen on kainic acid induced reduction in hippocampal 
GABA release in mice. Mice were administered melatonin $(10 \mathrm{mg} / \mathrm{kg}$, i.p. $) 45$ minutes prior to and immediately following kainic acid (10 mg/kg, s.c.) treatment and every 6 hours thereafter until conclusion of study. Other animals received either vitamin $\mathrm{E}$ (500 $\mathrm{mg} / \mathrm{kg}$, intragastric) or estrogen $(500 \mathrm{mg} / \mathrm{kg}$, subdermic) following kainic acid injections. All mice were given kainic acid injections once daily for 7 days.

Ortiz et al. found that all of the antioxidants administered (melatonin, vitamin E, and estrogen) prevented the kainic acid from reducing potassium evoked hippocampal GABA release (2001). Melatonin was found to be more effective than vitamin $\mathrm{E}$ in protecting against kainic acid induced oxidative damage.

\section{Melatonin is neuroprotective in stroke models}

Several studies have provided evidence for the increase of oxygen-radical reactions during ischemia (Siesjo, 1988; Zini et al., 1992). During ischemia, a number of events occur that tends to favor the production of free radicals and lipid peroxidation. Ischemia results in energy depletion that produces an excess release of the excitatory neurotransmitter, glutatmate. This then results in the overstimulation of the glutamate receptors that activates toxic $\mathrm{Ca}^{2+}$ dependent and independent pathways that ultimately leads to the production of free radicals.

A number of studies have assessed the efficacy of melatonin in counteracting neurodegeneration in animal models of acute stroke. Manev et al. (1996) examined the effects of melatonin deficiency in rats that underwent middle cerebral artery occlusion (MCAO). Fifteen days following pinealectomy both common carotid arteries and the left middle cerebral artery were occluded with microsurgical clips for 1 hour. Following 1 
hour of occlusion, blood flow was restored by removal of the clips. Manev et al. (1996) found that MCAO resulted in greater brain damage in the pinealectomized rats compared to the sham operated animals. Using a computer assisted image analysis system, Manev and colleagues measured the infarct volumes on Nissl stained coronal sections (40 um thick). A modest loss of Nissl staining in the left frontoparietal cortex was observed 4 hours following MCAO. Quantitative analysis revealed that the pinealectomized animals exhibited greater lesion volume than sham-pinealectomized animals. Thus, lowering the level of endogenous melatonin exacerbated the neural effects of acute MCAO.

In a later study by these investigators, they examined the effect of pinealectomy on the consequences of reversible cerebral artery occlusion (CerAO) in rats (Joo et al. 1998). These investigators also examined in pinealectomized animals, the neuroprotectant effects of intraperitoneal injections of melatonin $(2.5 \mathrm{mg} / \mathrm{kg}$, i.p. $)$ against the consequences of CerAO. Reversible focal ischemia was produced by occluding the middle cerebral artery (MCA) with a vascular clamp and occluding both common carotid arteries (CCA) with nylon sutures. Following 90 minutes of this three-vessel occlusion, recirculation was established by deoccluding the MCA and both CCAs. Three different reperfusion times $(4,6$, or $24 \mathrm{hr})$ were used to ascertain the time course of CerAOinduced brain damage. To assess the neuroprotective effects of melatonin, rats were injected with melatonin 30 minutes prior to occlusion, immediately following recirculation of blood flow, and 1 and $2 \mathrm{hr}$ later. A group of pinealectomized rats received saline injections thus serving as a control group.

Joo and colleagues found that for all reperfusion time points, DNA damage as assessed by the TUNEL technique, was greater in the pinealectomized rats compared to 
the non-pinealectomized animals. Moreover, infarct volume was increased in pinealectomized rats. The administration of melatonin to pinealectomized animals resulted in a reduction of TUNEL-positive cells and a reduction in lesion volume.

In a later study, Cuzzocrea et al. (2000) examined the effects of melatonin treatment in protecting against ischemia and reperfusion injury in gerbils. Brain ischemia and reperfusion damage was effected by occluding both carotid arteries for 5 minutes. Melatonin treated gerbils received an injection of melatonin $(10 \mathrm{mg} / \mathrm{kg}$, i.p.) 30 minutes prior to reperfusion and 1,2, and 6 hours following blood reflow. The vehicle treated animals that served as ischemic controls, received saline instead of melatonin injections. Another group of animals received identical surgical procedures as the ischemic group except that the carotid arteries were not occluded. In addition to this sham group, another sham group received melatonin injections similar to the ischemic treated animals.

Cuzzocrea and colleagues found that the untreated ischemic gerbils exhibited an increase in lipid peroxidation as shown by increased MDA levels 60 minutes following recirculation (2000). Treatment with melatonin reduced this increase in MDA levels. Melatonin administration also attenuated the hippocampal increase of myeloperoxidase (MPO), an indicator of polymorphonuclear leukocytes (PMN's) following reperfusion. Ischemia and reperfusion also significantly reduced hippocampal CA1 pyramidal cells. This cell loss was attentuated in gerbils who had been treated with melatonin.

In a similar study, Sinha and colleagues (2001) assessed the ability of melatonin to protect against ischemic reperfusion damage in rats. Cerebral ischemia was induced by occluding the MCA with a nylon filament for 2 hours followed by reperfusion of the area for 2 hours. Rats were divided into five different groups. Sham animals underwent 
identical surgical procedures except the MCA was not occluded. Another sham group that received saline injections was also included. Ischemic groups received either saline injections or injections of melatonin at 10,20 , or $40 \mathrm{mg} / \mathrm{kg}$, i.p. All injections were given at the time of MCA occlusion, 1 hour following occlusion, immediately following recirculation, and 1 hour following reperfusion.

These investigators found that following 2 hours of blood reflow, MCA occluded rats showed an increase in MDA levels compared to sham operated animals. Rats who had received 20 and $40 \mathrm{mg} / \mathrm{kg}$ of melatonin showed significantly reduced MDA levels compared to the vehicle treated MCA occluded rats. By contrast, melatonin did not attenuate MDA levels at the $10 \mathrm{mg} / \mathrm{kg}$ dosage. In addition, all melatonin treated rats exhibited smaller ischemic lesions compared to vehicle treated rats.

In summary, administration of melatonin to MCA occluded rats resulted in a decrease of lipid peroxidation products and a reduction in the volume of the ischemic lesion. .

In a more recent study, Torii et al. (2004) examined the effectiveness of melatonin in protecting against cerebral edema caused by ischemia/reperfusion. Rats underwent $\mathrm{thr}$ of middle cerebral artery (MCA) occlusion followed by reperfusion. Melatonin was administered $(6 \mathrm{mg} / \mathrm{kg})$ just prior to transient MCA occlusion and 1 day following surgery. Using magnetic resonance imaging (MRI), these investigators found that melatonin significantly reduced cerebral edema caused by transient ischemia/reperfusion. The protective effect of melatonin in reducing cerebral edema volume was more pronounced in the cerebral cortex $(60 \%)$ compared to the striatum (34\%). The authors suggested that melatonin may provide an effective clinical treatment in protecting against 
the motor, sensory, memory, and psychological impairments associated with ischemic stroke.

Lee et al. (2004) examined whether melatonin afforded protection against the electrophysiological and neurobehavioral impairments observed following MCA occlusion/reperfusion in rats. Rats underwent $1.5 \mathrm{hr}$ of MCA occlusion followed by reperfusion. At the initiation of reperfusion, rats were administered intravenous injections of either melatonin $(5 \mathrm{mg} / \mathrm{kg})$ or a vehicle (distilled water). Lee et al. found that intravenous melatonin administration significantly reduced both cortical and striatal infarct sizes and was effective in improving neurobehavioral outcome.

All of the aforementioned studies examined the effect of melatonin administration and/or melatonin deficiency on acute ischemic models. To date, only one study, carried out by our laboratory, has examined the long-term effects of pinealectomy on the behavioral and neuropathological consequences of chronic, moderate brain ischemia in the rat (De Butte et al., 2002). Pinealectomized and non-pinealectomized animals were trained on a tactile radial arm maze. Following training, they underwent permanent occlusion of the common carotid arteries (2VO) or sham surgery followed by re-testing in the RAM task and then neurohistological assessment at 16 months.

De Butte et al. (2002) found that pinealectomized 2VO animals exhibited a greater number of working memory errors compared to both the non-pinealectomized 2VO and pinealectomized non-2VO groups. Hence, combined pinealectomy and 2VO resulted in a significant working memory impairment. Counts of hippocampal CAl and CA4 pyramidal cells using stereological techniques revealed that $2 \mathrm{VO}$ alone resulted in a $10 \%$ reduction of CA1 hippocampal cells. Pinealectomy alone resulted in a $21 \%$ 
reduction. Pinealectomized $2 \mathrm{VO}$ animals showed the greatest reduction (32\%) of CA1 cells. Similar results were seen in the hippocampal CA4 region. Pinealectomized animals also exhibited greater glial fibrillary protein immunoreactivity in both hippocampal CA1 and $\mathrm{CA} 4$ regions.

The main findings of this study were that not only did pinealectomy exacerbate the consequences of chronic brain ischemia but it also caused hippocampal damage. In the same report, De Butte et al. (2002) found in a separate cohort of pinealectomized rats that no cell loss was observed 2 weeks following pinealectomy. This finding is important because it suggests that the effects of pinealectomy were either slow to evolve or precipitated by aging.

The only other study to examine the effect of chronic pinealectomy, determined if it would result in an increase in measures of oxidative stress as these animals aged (Reiter et al. 1999). At two months of age, rats underwent either pinealectomy or sham surgery. These animals were allowed to age to 25 months after which these investigators assessed levels of lipid peroxidation products and oxidatively damaged DNA in various tissues and compared these to young, two month old animals. Reiter and colleagues examined liver, lung, pancreas, kidney, testis, muscle, skin, and brain tissue in these animals. The brain was divided into cerebral cortex, cerebellum, hippocampus and brain stem.

In order to assess oxidative damage to DNA, these investigators measured 8hydroxy-2-deoxyguanosine (8-OHdG) levels in the liver, kidney and pancreas. 8-OHdg is a modified base that occurs in DNA due to attack by hydroxyl radicals. Hence, 8 -OHdg has become increasingly popular as a sensitive, stable and integral marker of oxidative damage in cellular DNA. Compared to the young animals, the old sham-pinealectomized 
animals exhibited higher levels of 8-OHdG. These increases were further augmented by pinealectomy. MDA and 4-HDA levels were measured as an indicator of lipid peroxidation. Aging was associated with a significant augmentation of MDA and 4-HDA levels in the lung, kidney, and skin. By contrast, no significant increases were found in pancreas, testes, muscle, or any of the brain regions assessed. It was found however, that pinealectomized animals had significantly higher MDA and 4- HDA levels in the lung, pancreas, testes, muscle, brain stem and hippocampus compared to the old sham controls. This study demonstrated that life-long pinealectomy exacerbated the oxidative damage that a number of tissues including the brain, sustain with aging, arguably as a result of the lowering of endogenous melatonin and its associated antioxidative and free radical scavenging properties.

According to the free radical theory of aging, the accumulation of free radical induced damage to proteins, lipids, and DNA over an individual's lifetime may account for aging processes and susceptibility to age-related diseases (Reiter, 1995; Reiter, 1997; Srinivasan, 1999). An age-related decline of melatonin levels in some individuals may lower endogenous free radical scavenging ability, thus contributing to age-related pathological processes. If melatonin either potentiates endogenous free radical defense mechanisms or directly acts as an antioxidant, then it is not surprising that chronic pinealectomy would cause the loss of CA1 and CA4 pyramidal cells with aging (De Butte et al., 2002) and increase age-related oxidative damage (Reiter, 1999).

To date, no study has attempted to reverse the effects of chronic pinealectomy by melatonin replacement. Moreover, studies involving pinealectomy have either been shortterm $(<4$ weeks) or have been quite chronic $(>16$ months). Furthermore to date, no study 
has examined the behavioral and neuropathological effects of pinealectomy at various time points to determine when these effects take place.

\section{Free Radicals, Melatonin, and Neurodegenerative Diseases}

Collectively, all of the studies reviewed have provided strong evidence that melatonin is a potent antioxidant and free radical scavenger. This finding has been of interest to researchers interested in neurodegenerative disorders. Damage to cells and tissues by toxic free radicals is believed to occur in a variety of neurodegenerative disorders such as Alzheimer's disease and Parkinson's disease (Reiter, 1998). Senile plaques, one of the main pathologic markers of Alzheimer's disease, contain depositions of the amyloid beta peptide (A $\beta$ ) (Miranda et al., 2000). Evidence suggests that $A \beta$ is capable of instigating a cascade of events that ultimately results in the production of free radicals that leads to damage of neuronal membrane lipids, proteins, and nucleic acids eventually causing cell death (Cotman, 1998; Miranda et al., 2000; Montine et al., 1997; Smith et al., 1996; Subbarao et al., 1990). Administration of A $\beta$ to cells in culture leads to an increase in hydrogen peroxide production which as previously described, is capable of generating the highly toxic hydroxyl radical when it reacts with transition metals (Behl et al., 1994b; Reiter, 1998).

The finding that free radicals may be involved in the neuropathology seen in Alzheimer's disease has led investigators to explore the efficacy of melatonin in treating neurodegenerative conditions such as Alzheimer's disease. Significantly, it has been found that plasma melatonin levels are reduced with increasing age (Reiter, 1995). Recent research further demonstrated that cerebrospinal fluid levels of melatonin were 
markedly reduced in Alzheimer's patients (Lui et al., 1999; Zhou et al., 2003). For example, Liu and colleagues (1999) determined post-mortem melatonin levels in the cerebrospinal fluid of a large sample of Alzheimer's patients $(\mathrm{N}=85)$ and age-matched controls $(\mathrm{N}=82)$. A significant decrease in melatonin was found with age. Older control patients exhibited a $50 \%$ reduction in melatonin levels compared to younger controls. Additionally, melatonin levels in the cerebrospinal fluid of Alzheimer's patients showed a five-fold reduction compared to age-matched controls.

In a later study, Zhou and colleagues (2003) found that cerebrospinal fluid levels of melatonin were significantly reduced in Alzheimer's patients compared to controls. Interestingly, the levels of melatonin were also correlated with the severity of the disease. Zhou and colleagues (2003) found that patients who exhibited early neuropathological changes also exhibited a significant reduction in melatonin. This suggests that a reduction in melatonin may occur in the early stages of Alzheimer's disease and may even precede the advent of clinical symptoms. These experiments were notable because of their large sample sizes and the fact that all of the patients were neuropathologically confirmed.

As previously mentioned, considerable evidence exists showing melatonin's efficacy as a free radical scavenger and antioxidant. Because damage from free radicals is thought to occur in Alzheimer's disease, several studies have investigated the neuroprotective role of melatonin in models of Alzheimer's disease (Pappolla et al., 1998; Pappolla et al., 2000; Poeggeler et al., 2001). In several in vitro studies conducted by Pappolla and colleagues $(1997,1998,1999)$, melatonin was capable of protecting cultured neuroblastoma cells and primary cell neurons against $A \beta$ exposure. Interestingly, Pappolla and colleagues (1998) further demonstrated that melatonin inhibited the 
formation of $\beta$-sheets and amyloid fibrils. These in vitro studies were noteworthy because they suggested that melatonin not only exhibited antioxidative properties but also exhibited antiamylidogenic properties.

In addition to the in vitro studies just described, melatonin was demonstrated to have neuroprotective properties in an in vivo model of Alzheimer's disease (Matsubara et al., 2003). Using a transgenic mouse model of Alzheimer's disease, Matsubara and colleagues (2003) investigated whether melatonin was capable of affording protection against the age-related augmentation of $\mathrm{A} \beta$ peptides and abnormal protein nitration. Melatonin replacement in the drinking water significantly reduced $A \beta$ levels and protein nitration in brain tissue of the transgenic mice. Additionally, melatonin treatment also markedly enhanced the survival of these mice. This study is the first to address whether there is a relationship between melatonin and the biochemical neuropathology of Alzheimer's disease using an in vivo model.

In addition to Alzheimer's disease, the involvement of free radicals has been postulated in the etiology of Parkinson's disease (Alam et al., 1997; Reiter, 1998; Lohr, 1991). Parkinson's disease involves a progressive neurodegeneration of dopaminergic cells in the substantia nigra resulting in sensory and motor dysfunction. Specifically, patients exhibit a resting tremor, bradykinesia, and rigidity. The free radical theory of Parkinson's disease asserts that death of dopaminergic neurons is due to both the production of free radicals as a result of catabolic pathways of dopamine, and also a result of the auto-oxidation of dopamine (Reiter, 1998). Numerous studies have found free radical mediated injury to lipids (Yoritaka et al., 1996), proteins (Alam et al., 1997), and nucleic acids (Alam et al., 1997) in the substantia nigra of Parkinson's patients. 
The most common animal model of Parkinson's disease involves the cytotoxin 1methyl-4-phenyl-1,2,3,6-tetrahydropyridine (MPTP). Administration of MPTP in rodents results in the production of free radicals, energy depletion of cells and ultimately, apoptotic cell death (Reiter, 1998). Several studies have investigated whether melatonin was neuroprotective against MPTP neurotoxicity and these have found that melatonin is efficacious in reducing the effects of MPTP administration. Specifically, melatonin adminstration to MPTP treated mice has been found to prevent nigral cell death (Antolin et al., 2002), significantly reduce lipid peroxidation levels in the striatum, hippocampus, and midbrain (Acuna-Castroviejo et al., 1997), and block the reduction of tyrosine hydroxylase immunoreactivity (Acuna-Castroviejo et al., 1997; Antolin et al., 2002; Jin et al. 1998). The finding that melatonin is protective in animal models of neurodegenerative conditions, is suggestive that this indole may be of therapeutic value in treating these conditions.

\section{Present Studies}

Taken together, there is a substantial amount of research demonstrating melatonin's potent neuroprotective properties. Further evidence that melatonin is a neuroprotectant in the brain has come from recent studies demonstrating that pinealectomy and the resultant lowering of endogenous melatonin, exacerbated the effects of acute and chronic ischemia and itself causes the loss of CA1 and CA4 hippocampal pyramidal cells (De Butte et al., 2002; Manev et al., 1996). However this loss of pyramidal cells was noted at 16 months post-pinealectomy, which was performed when the rats were about one month of age. There is no information regarding the time 
course of this effect. Does it slowly evolve or manifest with aging? The current study was designed to address this.

The present study examined the effects of $2,4,6,8,10$ and 17 months of pinealectomy using both behavioral and neuropathological measures such as spatial learning and markers of neuronal integrity respectively. Because many different time points were chosen, this study allowed for the determination of when any neuropathological changes might take place.

In addition, the behavioral effects of pinealectomy were examined. These have yet not been fully elucidated. Most of the studies involving pinealectomized animals were short- term and did not involve testing these animals behaviorally. To date, we are the only laboratory that has tested these animals on a spatial learning task (Tactile RAM). Thus, the behavioral consequences of pinealectomy if any, needed to be clarified. It is well known that hippocampal damage in rats impairs spatial learning and memory (Olton \& Papas, 1979). One of the memory systems particularly vulnerable to hippocampal damage is working memory, the memory system responsible for the remembering of trial-specific events. Our previous research indicated that pinealectomy by itself causes hippocampal cell loss (De Butte et al., 2002). In order to fully characterize if there are functional behavioral consequences of this cell loss, animals were tested here on a working memory version of the Morris water maze (MWM), as this task is sensitive to hippocampal damage (Lin \& Bilkey, 2001). Animals were tested at different time-points $(2,7$, and 11 months after pinealectomy) in order to determine the time course of any effect of pinealectomy on this task. 
As previously described, in addition to its antioxidant properties, melatonin has also been found to have many psychopharmacological effects in rodents such as sedation, analgesia, and anxiolytic properties (Kopp et al., 1999). No study has tested whether long-term pinealectomized animals are more anxious. Hence, anxiety was also measured in these animals using the elevated plus maze. The elevated plus maze task is one of the most widely used tasks for measuring anxiety in animals (Pellow et al., 1985). The measure of fearfulness (anxiety) is the proportion of time that rats spend in the enclosed arms, rather than venturing out onto the exposed arms. In addition to indexes of anxiety, the elevated plus maze also allows for the measurement of several other behaviors such as locomotion, exploration, and conflict decision resolution (Andrade et al., 2003).

In order to determine the time course of neuron loss following pinealectomy, the rats' brains were examined at systematic intervals $(2,4,6,8,10$, and 17 months) following pinealectomy. The extent of cell loss was determined by using stereological counting techniques. Stereology allows for the determination of an unbiased estimate of total cell number in a brain structure, without having to make assumptions about their size, shape, and orientation (West, 1994). The technique prevents the duplicate counting of cells in consecutive sections, and when combined with systematic sampling, it allows for the reliable estimation of total cell number. In addition to stereology, Fluoro-Jade B staining was undertaken in order to detect cells that were in the process of dying. It was predicted that pinealectomy would result in an increase in reactive oxygen species, followed by an increase in cell loss and an increase in Fluoro-Jade B staining. Because there is an accumulation of free radical products that occurs as a result of the aging process, it was expected that these changes should be more apparent as these animals age. 
A second major purpose of this study was to examine the effects of melatonin supplementation on animals that underwent $2,4,6,8,10$, or 17 months of pinealectomy. This group was essential in determining if any effects of pinealectomy were specifically due to the loss of melatonin. There is very little research involving chronic melatonin supplementation in pinealectomized rats. Rasmussen et al (1999) reported that 10 weeks of treatment with melatonin $(4 \mathrm{mg} / 1)$ in the drinking water of normal 10 -month rats resulted in a nocturnal plasma level that was 15 times higher than younger animals. In a more recent study, Regrigny et al (2001) administered $6 \mathrm{mg} / \mathrm{l}$ of melatonin for one month to young control and pinealecotmized animals. These investigators reported that pinealectomy reduced cerebral arteriolar diameter and this was prevented by melatonin supplementation. Using the dosage used in the Regrigny et al study, it was expected that the effects of pinealectomy here would be reversed by melatonin replacement, thus providing additional support that melatonin has neuroprotective properties. It was predicted that melatonin replacement would reverse the behavioral and neuropathological consequences of pinealectomy.

A third major purpose of this experiment was to assess free radical activity and endogenous antioxidant activity in short-term ( 3 month) and long-term (20 month) pinealectomized animals by measuring lipid peroxidation products and glutathione (reduced and oxidized forms). It was hypothesized that pinealectomized animals would exhibit an elevation of lipid peroxidation products in the hippocampus as this region was expected to show cell loss like that previously noted by our laboratory (De Butte et al., 2002). In contrast, it was hypothesized that the animals that received melatonin supplementation would not exhibit this elevation in lipid peroxidation products. It was 
further hypothesized that all animals at the 20 month time-point would exhibit significantly more lipid peroxidation products compared to the 3 month animals. It was also hypothesized that pinealectomized animals at both the 3 and 20 month time-points would exhibit significantly higher oxidized glutathione (GSSG) and that melatonin supplemented animals were hypothesized to exhibit lower GSSG levels and that melatonin supplementation would reverse these effects. 


\section{Methods}

\section{Animals}

One-month old pinealectomized $(\mathrm{N}=69)$ and sham-pinealectomized $(\mathrm{N}=36)$ animals were obtained from Harlan Sprague-Dawley (Indianapolis, IN). We purchased one-month old pinealectomized and sham-pinealectomized rats from Harlan-Sprague Dawley for several reasons. First, the mortality rate from this surgery can be high due to the puncturing of the sagittal and transverse sinuses, under which the pineal lies. While we are able to perform the surgery, we do lose animals and in view of the large numbers needed for this study, the cost of this was a burden. The breeder chargers only for animals that survived the procedure and the cost of the surgery per animal was modest. Hence, it was much more economical to purchase pinealectomized rats. The breeder has also been diligent in implementing our recommended modifications to the procedures for sham controls.

The pinealectomy surgery was performed by the Harlan surgeon 1 week prior to our receiving the animals. Briefly, the surgery was as follows. Animals were anaesthetized with a ketamine $(5 \mathrm{mg} / \mathrm{kg})$ and xylazine $(1 \mathrm{mg} / \mathrm{kg})$ cocktail. They were then placed in a stereotaxic instrument and a 1-1.5 midline skull incision was made. The underlying connective tissue was removed by scraping the skull with a blunt probe. A small hole was then drilled above the right cerebral hemisphere near the junction of the superior sagittal sinus and the transverse sinus. A small cut was then made in the exposed dura. The pineal was removed using a pair of blunt-tipped curved iris forceps. The incision was closed with surgical glue. Sham-pinealectomized animals underwent the identical procedure except the pineal gland was not removed. The surgeon was informed 
of the need for the sham procedure to be as identical to the pinealectomy as possible and to avoid trauma to the brain tissue near the pineal.

Animals were randomly divided into 6 groups that represented differing survival times. The first group was sacrificed 2 months following pinealectomy $(\mathrm{N}=13)$ or shampinealectomy surgery $(\mathrm{N}=9)$. The second group was sacrificed 4 months following pinealectomy $(\mathrm{N}=12)$ or sham-pinealectomy $(\mathrm{N}=6)$ surgery. The third group was sacrificed 6 months following pinealectomy $(N=12)$ or sham-pinealectomy $(N=6)$ surgery. The fourth group was sacrificed 8 months following pinealectomy $(\mathrm{N}=12)$ or sham-pinealectomy $(\mathrm{N}=6)$. The fifth group was sacrificed 10 months following pinealectomy $(\mathrm{N}=10)$ or sham-pinealectomy $(\mathrm{N}=4)$. The final group was sacrificed 17 months following pinealectomy $(\mathrm{N}=10)$ or sham-pinealectomy $(\mathrm{N}=5)$. These groups allowed for the determination of the effects of pinealectomy at systematic intervals including long term. In addition, some of the pinealectomized animals $(\mathrm{N}=37)$ at each time point received melatonin replacement in their drinking water. The melatonin supplemented animals were sacrificed either 2 months $(\mathrm{N}=9), 4$ months $(\mathrm{N}=6), 6$ months $(\mathrm{N}=6), 8$ months $(\mathrm{N}=6), 10$ months $(\mathrm{N}=5)$ or 17 months $(\mathrm{N}=5)$ post-pinealectomy. Animals from each experimental group were also randomly divided into 3 groups for behavioral testing at three time-points. The first groups of sham-pinealectomized $(\mathrm{N}=10)$, pinealectomized $(\mathrm{N}=10)$ and melatonin supplemented $(\mathrm{N}=10)$ animals were tested on the Morris water maze (MWM) and the elevated plus maze tasks 2 months postpinealectomy or sham-pinealectomy. The second group of sham-pinealectomized $(\mathrm{N}=10)$, pinealectomized $(\mathrm{N}=10)$ and melatonin supplemented $(\mathrm{N}=10)$ animals were tested on the MWM and the elevated plus maze tasks 7 months post-pinealectomy or sham- 
pinealectomy. The final group of sham-pinealectomized $(\mathrm{N}=10)$, pinealectomized $(\mathrm{N}=10)$, and melatonin supplemented $(\mathrm{N}=10)$ animals were tested on the MWM and the elevated plus maze tasks 11 months post-pinealectomy or sham-pinealectomy. In addition to these groups, an additional subset of sham pinealectomized $(\mathrm{N}=10)$, pinealectomized $(\mathrm{N}=10)$, and melatonin supplemented $(\mathrm{N}=10)$ animals were tested on the elevated plus maze task 15 months following surgery. Animals were individually housed on a 12-hour light/dark cycle (lights on 20:30, off 8:30) and received food and water $a d$ lib.

\section{Melatonin Administration}

Melatonin (Sigma Chemical CO, St. Louis, MO) was dissolved in ethanol and added to the drinking water. Melatonin was prepared three times a week by dissolving the drug $(0.12 \mathrm{mg})$ in ethanol $(1 \mathrm{ml}, 100 \% \mathrm{vol} / \mathrm{vol})$. This solution was then diluted with distilled water to a final concentration of $6 \mathrm{mg} / \mathrm{l}$ (final concentration of ethanol, $0.05 \%$ ). The average daily intake of melatonin was $0.51 \pm 0.02 \mathrm{mg} \cdot \mathrm{kg}$ day. The dose of melatonin that was used in the present study was chosen on the basis of a previous report (Regrigny et al. 2001) that demonstrated this dose was sufficient to produce significantly higher plasma melatonin levels than in untreated pinealectomized animals. The melatonin solution was given to rats in opaque water bottles because melatonin in solution will degrade when exposed to light. 


\section{Behavior}

Elevated Plus Maze Testing

The apparatus consisted of two open arms $(52 \times 12 \mathrm{~cm})$ opposite each other and two enclosed (side and end walls) arms opposite to one another $(52 \times 12 \times 40 \mathrm{~cm})$ (Pellow et al., 1985). The open and closed arms were perpendicular to one another, intersecting at their centers. Both arms were constructed of opaque white polyvinyl chloride. The maze was elevated $50 \mathrm{~cm}$ above the ground. A video camera was hung above the maze to allow for recording.

Each rat was positioned at the arm intersection and pointed to either randomly chosen open or closed arms of the maze. The rat was then allowed to explore the maze for 10 minutes. Anxiety behaviors that were recorded consisted of (1) open entries (2) open arm entry ratio (3) open arm time ratio and (4) ratio of stretch attend postures from protected area (Andrade et al., 2003; Pellow et al., 1985; Wall \& Messier, 2001). In addition, a decision index was assessed by recording the ratio of time spent in the central hub (Andrade et al., 2003). Locomotor activity was measured by recording the number of total entries and closed arm entries. Finally, exploration was measured by recording the total number of head dips in the open arms and number of vertical stretches in the closed arms. The rats underwent a single test in this maze. 


\section{Morris Water Maze (MWM) Testing}

\section{Apparatus}

The maze consisted of a large white polypropylene pool $1.6 \mathrm{~m}$ in diameter that was filled approximately $15 \mathrm{~cm}$ from the top with tepid water. Large black visual cues were placed on the walls of the room to facilitate spatial mapping.

\section{MWM training}

Rats were given 1 session per day for a total of 6 sessions over a 6-day period on the working memory version of the MWM. A clear plastic platform was placed approximately $2 \mathrm{~cm}$ below the water line in one of the four quadrants of the pool. The location of the platform was changed at the start of every daily session but remained in that quadrant for all trials on that particular day. A training trial consisted of a forced swim from one of several starting points (north, northeast, northwest, south, southeast, southwest, east, and west) giving a total of five swims per session. The sequence of the five start positions varied for each session. Each animal was given 90 seconds to reach the platform and had to remain on the platform for 10 seconds. If the platform was not located within the 90 seconds, the animal was guided to the platform by the experimenter. Latency to find the hidden platform was recorded for each swim and the latency from the last four trials was averaged for each daily session. Distance to the platform was recorded for each swim and the distance from the last four testing trials was averaged for each daily session. A tracking system was used to calculate latency to find the platform and distance (SMART, San Diego Instruments). 


\section{Histological Methods}

Animals in the 2-month group were sacrificed 2 months post-surgery. Animals in the $4,6,8,10$ and 17 - month groups were sacrificed at $4,6,8,10$, and 17 months postsurgery respectively. All animals were sacrificed by sodium pentobarbital overdose. Rats were transcardially perfused with $75 \mathrm{ml}$ heparinized saline followed by $500 \mathrm{ml} 4 \%$ paraformaldehyde in $0.1 \mathrm{M}$ phosphate buffer, $\mathrm{pH} 7.4$.

Upon removal of the brain, the presence or absence of the pineal gland was verified by visual inspection. The brains were first cut coronally separating the cerebellum, and then the two cerebral hemispheres were separated at the midline. The right hemisphere was washed in 0.1 phosphate buffer, $10 \%$ sucrose, and then $20 \%$ sucrose in preparation for cryostat sectioning. Forty micron coronal sections were floated in a cryoprotectant solution ( $37.5 \%$ sucrose, $37.5 \%$ glycerol inn $0.1 \mathrm{M}$ phosphate buffer) in polystyrene well plates and stored at $-20{ }^{\circ} \mathrm{C}$ for future analyses.

The left hemisphere was post-fixed in a fixative solution for 24 hours at $4{ }^{\circ} \mathrm{C}$. Following post fixation, the tissue was rinsed in 0.1 phosphate buffer plus azide, pH 7.4. The first rinse was for 4 hours and the second rinse overnight. The brains were then dehydrated in graded alcohols, cleared in clearene and then paraffin embedded. Forty micron saggital sections containing the hippocampus were sectioned with a microtome.

In order to visualize hippocampal pyramidal cells, 40 micron paraffin sections were stained with hematoxylin. Briefly, sections were de-paraffinized and then were incubated in Gill's hematoxylin for 30 minutes. Sections were then placed under running lukewarm water for an additional 20 minutes and then dehydrated, cleared and coverslipped. 
A set of 3 forty micron paraffin sections containing the hippocampus were also stained with Fluorojade B (Histo-Chem) a marker of neurodegeneration (Schmued \& Hopkins, 2000). Tissue sections were first de-paraffinized and then placed in $100 \%$ alcohol for 3 minutes after which they were then placed in $70 \%$ and $30 \%$ alcohol for 1 minute each. Sections were then rinsed in distilled water for 1 minute. Sections were then incubated at room temperature for 15 minutes in $0.06 \%$ potassium permagenate. Sections were then rinsed briefly in distilled water. Sections were then put in a $0.001 \%$ fluorojade solution in $0.1 \%$ acetic acid for 30 minutes. Sections were dried on a slide warmer for 10 minutes and then briefly placed in zylene after which they were coverslipped with D.P.X. mounting medium.

\section{Hippocampal Measures}

Measures for the determination of hippocampal CA1, and CA3 pyramidal cell counts were derived using systematic random sampling techniques. Briefly, this involved selecting sections at a regular interval (every sixth section) along the entire hippocampus after the first section had been selected at random from the first interval. All microscopic analyses were conducted on an Olympus BH-2 microscope.

Hippocampal cell counts of hematoxylin stained sections was carried out using standard stereological techniques (West, Slomianka, \& Gundersen, 1991). Neurons were counted using stereo investigator software (Micro-Brightfield Inc) that uses the optical fractionator method consisting of the use of a three-dimensional probe known as the optical disector. 
Hippocampal cell counts of Fluorojade B reactive cells were carried out by manually counting the number of fluorescent stained cells in the CA1, CA3, CA4, and dendate gyrus. Cells were visualized at 40X magnification using an Olympus BX51 fluorescent microscope fitted with a 450nm FITC filter.

\section{Biochemical Analyses}

Animals

One-month old pinealectomized $(\mathrm{N}=28)$ and sham-pinealectomized $(\mathrm{N}=15)$ were obtained from Harlan Sprague-Dawley (Indianapolis, IN). The pinealectomy surgery was performed as previously described.

Animals were randomly divided into 2 groups representing differing survival times. The first group was sacrificed 3 months following sham-pinealectomy $(\mathrm{N}=7)$ or pinealectomy $(\mathrm{N}=13)$ surgery. The second group was sacrificed 20 months following sham-pinealectomy $(\mathrm{N}=8)$ or pinealectomy $(\mathrm{N}=15)$ surgery. Some of the pinealectomized animals $(\mathrm{N}=15)$ also received melatonin replacement in their drinking water as previously described. The melatonin supplemented animals were sacrificed either 3 months $(\mathrm{N}=7)$ or 20 months $(\mathrm{N}=8)$ post-pinealectomy. Animals were individually housed in a 12-hour light/dark cycle (lights on 20:30, off 8:30) and received food and water ad $l i b$.

\section{Tissue Collection}

Animals in the 3-month group were sacrificed 3 months following shampinealectomy or pinealectomy surgery. The animals in the 20 -month group were 
sacrificed 20 months following sham-pinealectomy or pinealectomy surgery. All animals were exposed to a lethal dose of $\mathrm{CO}_{2}$ and were then decapitated. The brains were removed and for the 20-month animals, the cortex, hippocampus and a portion of the right kidney were dissected out. For the 3-month animals, only the hippocampus was dissected out. All tissue samples were frozen on dry ice and then transferred to a -80 freezer until analyzed.

\section{Thiobarbituric Acid Reactive Substances (TBARS) Determination}

Tissue samples were homogenized in $0.2 \%$ phosphoric acid (1:5, w:v). A second volume of $2 \%$ phosphoric acid was then added to the mixture. Two $400 u$ laliquots of the homogenate were collected and placed in glass tubes. To one tube, $400 u 1$ of TBA solution (1\% TBA in $50 \mathrm{mM} \mathrm{NaOH}$ with $500 u 1$ of $10 \mathrm{mM} \mathrm{BHT}$ ), and $7 \%$ phosphoric acid was added. To the other tube labeled blank, $400 u$ l of $3 \mathrm{mM} \mathrm{HCL}$ and $7 \%$ phosphoric acid was added.

Once the samples were checked for a $\mathrm{pH}$ of 1.6 , the tubes were placed in boiling water for 15 minutes. Boiling changes the sample solution into a red-pink color that is a result of the reaction of the aldehydes with TBA. After 15 minutes of boiling, the tubes were removed and were cooled to room temperature. Once at room temperature, $1.5 \mathrm{ml}$ of butanol was added to each tube. The tubes were then vortexed vigorously for 40 seconds and then centrifuged at $1000 \mathrm{rpm}$ for 5 minutes. Following centrifugation, there was an aqueous solution in the bottom of all tubes, and an organic (red-pink) solution on the top. An aliquot $(500 u l)$ of the organic layer was collected in a $1.5 \mathrm{ml}$ centrifuge tube. 
The absorption of the organic layer was measured for both sample and blank aliquots for each rat. Absorption at wavelengths between $400 \mathrm{~nm}$ and $600 \mathrm{~nm}$ were determined using a Cary 100 Bio UV-Visible spectrophotometer. Final absorption values were calculated by subtracting absorbance value at $532 \mathrm{~nm}$ by absorbance value obtained at $600 \mathrm{~nm}$. The data was represented in terms of $\mathrm{nmol} / \mathrm{g}$ wet tissue.

Glutathione (GSH) and Glutathione Disulfide (GSSG) Determination

Tissue samples were homogenized in $100 u$ of ice-cold $5 \%$ sulfosalicylic acid (pre-bubbled with $\mathrm{N}_{2(\mathrm{~g})}$ for 15 minutes) in an Eppendorf tube. Oxygen above the sample was removed by blowing $\mathrm{N}_{2(\mathrm{~g})}$ into the Eppendorf tube for 10 seconds and then the cap was quickly closed. The samples were then centrifuged at $13,000 \mathrm{~g}$ for 1 minute at $4^{\circ} \mathrm{C}$. The clear supernatant was transferred to a clean Eppendorf tube and $900 u 1$ of $0.5 \mathrm{M}$ potassium phosphate $(\mathrm{pH} \mathrm{6.0)}$ was quickly added in order to neutralize the acid. The pellet was then stored in a -80 freezer for protein determination later on.

Two microplates were prepared. One was kept on ice and contained the standards (GSH and GSSG) and samples (as well as glutathione reductase). The other housed the other components of the assay and was kept at room temperature. This microplate was used for the microplate readings.

For the GSH standards and samples (on ice), $1 \mathrm{mM} \mathrm{GSH}$ was diluted 1/20 with $0.5 \mathrm{M}$ potassium phosphate ( $\mathrm{pH} 6.0$ ). For the GSSG standards and samples (on ice), $100 u 1$ of the sample was collected and $20 u 1$ of 0.52 -vinylpridine that was diluted (dilution $=1.2 \mathrm{X}$ ) was added. These samples were then derivatized for 1 hour at room temperature. 
For the microplate assay (not on ice), $70 u l$ of $0.3 \mathrm{mM}(\mathrm{NADPH}), 10 u 1$ of $6 \mathrm{mM}$ (DTNB), $10 u \mathrm{l}$ of $50 \mathrm{IU} / \mathrm{ml}$ of glutathione reductase (added quickly), and $20 u l$ of sample or standard (added quickly) were added to the wells. The microplate was then read at 412 nm using a Spectra Max 340 PC microplate reader and the use of Softmax Pro computer software (Molecular Devices).

The Bio-Rad assay (Bio-Rad laboratories, Hercules, CA) was used for protein determination. Briefly, the pellet was resuspended in $100 u l$ of potassium phosphate $(\mathrm{pH}$ 6.0 ) and then centrifuged at $13,000 \mathrm{~g}$ for 5 minutes at $4^{\circ} \mathrm{C}$. The supernatant was then transferred to a new tube and the pellet was discarded. Using a 96 well-plate, $20 u 1$ of the supernatant and $150 u \mathrm{l}$ of the Bio-Rad solution was added to each well. The microplate was then read at $595 \mathrm{~nm}$ and using the BSA standard curve, the amount (in $u g$ ) of proteins in $20 u l(u g / u l)$ was found. The same procedure was used for the determination of GSSG. 


\section{Results}

\section{General Health and Weight}

Of the 148 animals that underwent sham- pinealectomy or pinealectomy surgery, 9 animals failed to complete the study. Seven pinealectomized animals died during the course of this study. Two of these animals were euthanized at the age of 13 months due to ill health. Two additional animals were euthanized at the age of 15 months due to ill health. Three more pinealectomized animals were euthanized at the age of 16 months due to ill health. In the sham-pinealectomized group, 2 animals died during the course of this study. One animal was euthanized at the age of 12 months while the other was euthanized at the age of 16 months as a result of ill health. No melatonin replacement animals died during the course of this study. All of the animals that died during the course of this study were animals whose brains were to be used for stereological and biochemical assessment at 17 and 20 months respectively. Specifically, 7 out of $20(35 \%)$ pinealectomized animals died while 2 out of $20(10 \%)$ sham- pinealectomized animals died. No melatonin supplemented rats died during the course of this experiment. A chi-square comparing the groups on the frequency of death was significant $\left(x^{2}=(2, N=60)=7.35, p<.05\right)$. Moreover, a comparison of the pinealectomized and melatonin supplemented groups revealed a significant difference in mortality rate $\left(x^{2}=(1,40)=6.23, p<.05\right)$. Melatonin reduced the death rate in pinealectomized rats from $35 \%$ to $0 \%$.

Weights were determined for the sham, melatonin supplemented, and melatonin deficient groups at 15-months of age. The group means were compared by of one-way 
analysis of variance (ANOVA) with group as a between subjects factor. As shown in Table 1 , the weights did not differ significantly among the groups.

\section{Behavior}

Elevated Plus Maze

Animals were given 1 session on the elevated plus maze task at 4 different timepoints $(2,7,11$, and 15 months). Behavior indices that were measured included those reflective of anxiety (number of open arm entries, percentage of entries on open arms/total arm entries, percentage of time on open arms/total time on maze, and percentage of protected stretch attend postures/total number of stretch attend postures), decision making (percentage of time spent on center hub/total time on maze), locomotor activity (number of total entries and number of entries in closed arms), and exploration (number of head dips in open arms and number of vertical stretches in closed arms). All ten measures were used for statistical analysis. For all time-points, a one-way ANOVA with Group (sham, melatonin supplemented, and pinealectomized) as a between subjects factor was performed. Post-hoc comparisons were performed using Tukey's test.

At 2 months, no significant group effect was observed for any of the anxiety measures. As illustrated in Figures 1 and 2 , the groups did not differ on the number of open arms entered, ratio of open arm entries, percentage of time spent in the open arms, or the percentage of protected stretch attend postures made from the closed arms.

A significant group effect was found on the decision index, percentage of time on center hub $(\mathrm{F}(2,27)=3.29, \mathrm{p}<.05)$. Tukey's test revealed that the pinealectomized animals 
Table 1. Group weights (in grams) for sham, melatonin supplemented, and pinealectomized rats. The groups did not differ in body weight. Data are means $\pm \mathrm{SE}$.

\begin{tabular}{|c|c|c|c|c|}
\hline Measure & Sham & Melatonin & Pineal & p \\
\hline Weight & $564 \pm 13.63$ & $529 \pm 15.90$ & $574 \pm 19.88$ & .19 \\
\hline
\end{tabular}


Figure 1. Top Panel: Open arm entries. The groups did not differ in the number of entries made into the open arms. Error bars represent S.E.M. Bottom Panel: Ratio of entries into the open arms. No differences were found between the groups on the percentage of entries made into the open arms. Error bars represent S.E.M. 

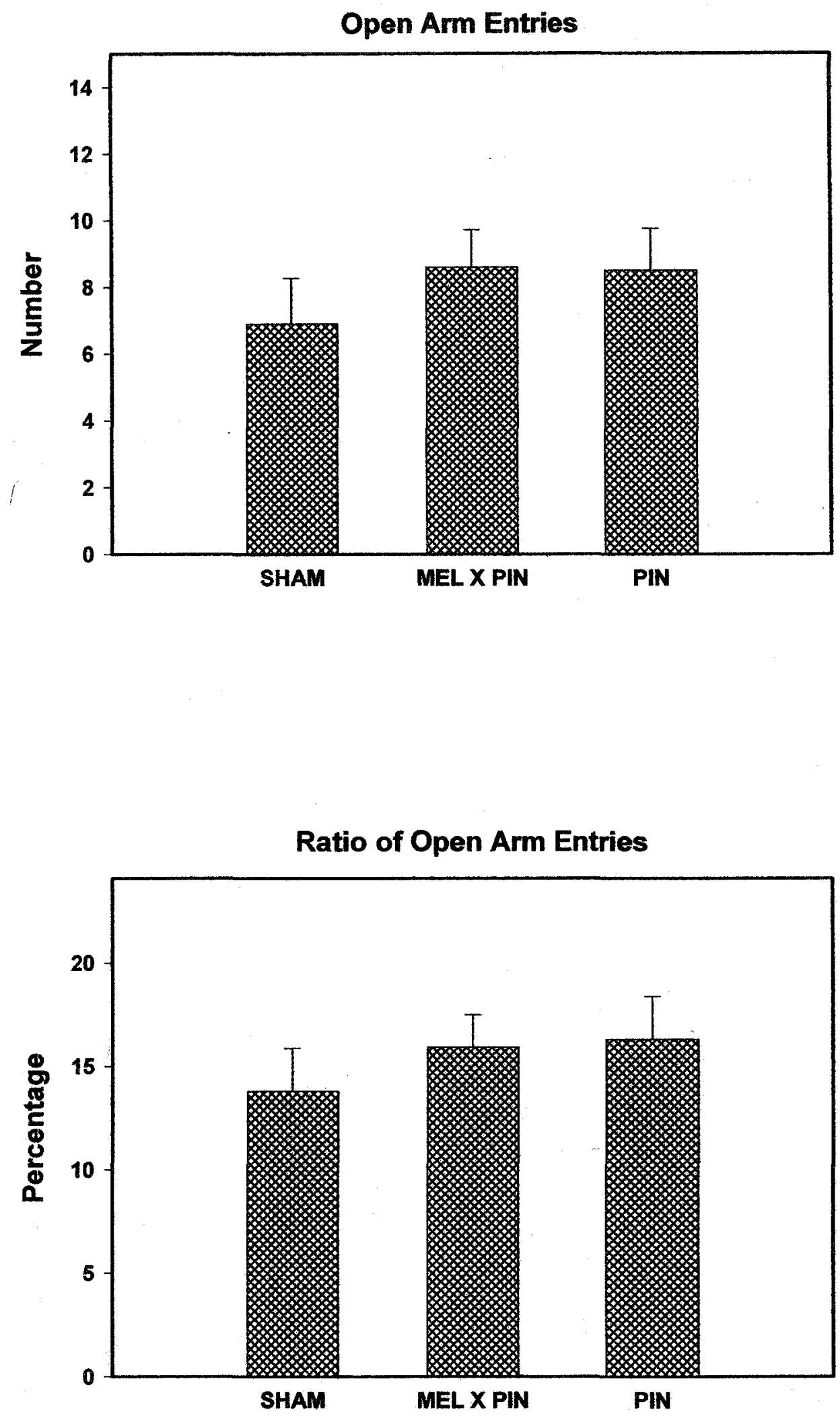
Figure 2. Top Panel: Percentage of time spent on open arms. The groups did not differ in the amount of time spent in the open arms. Error bars represent S.E.M. Bottom Panel: Ratio of protected stretched attend postures. No group differences were found in the percentage of stretch attend postures made from the closed arms. Error bars represent S.E.M. 

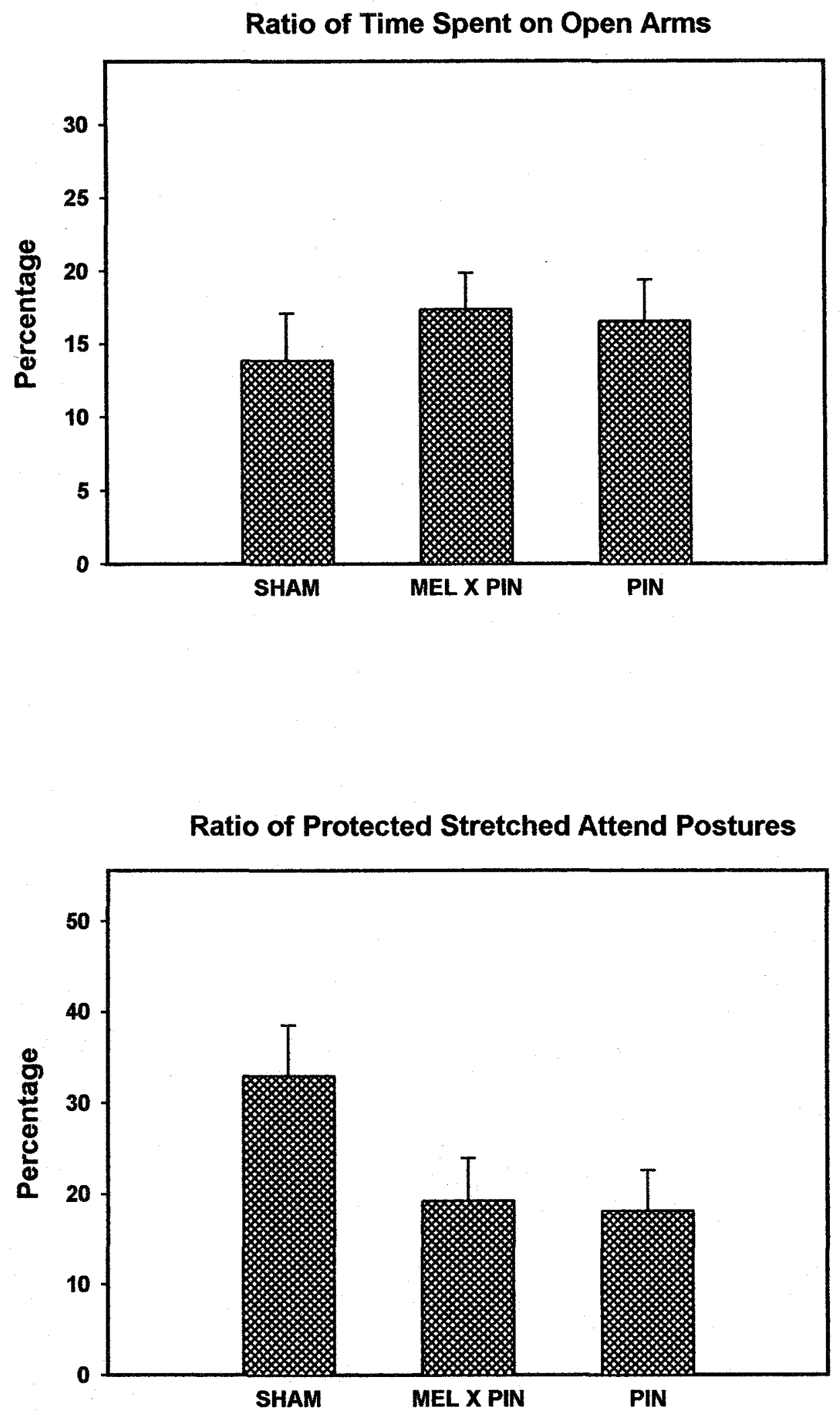
spent significantly more time in the central hub compared to the sham animals $(p<.05)$ (Figure 3). No significant differences in locomotor activity were observed among the three groups (Figure 4). The groups did not differ on the total number of arms entered or on the number of entries made into the closed arms. In addition, the groups did not exhibit any differences in exploration (Figure 5) on the maze. They did not differ on the number of head dips made in the open arms, or on the number of vertical stretches made in the closed arms.

At the 7-month time-point, no significant differences were observed between the three groups on any of the behavioral measures. The groups did not differ on any of the anxiety measures (Figures 6 and 7). Specifically, the rats did not differ on the number of entries made into the open arms, on the ratio of entries into the open arms, or in the percentage of time spent on the open arms. Moreover, the groups did not differ in the percentage of protected stretch attend postures made from the closed arms. The groups also did not exhibit any differences on the decision index (Figure 8). In addition, no significant differences were observed in locomotor activity or exploration on the maze (Figures 9 and 10). The groups did not differ on the total number of arms entered, or on the number of entries made into the closed arms. Moreover, the groups did not exhibit any differences on the number of head dips made in the open arms, or the number of vertical stretches made in the closed arms.

Similar to the 7-month rats, 11-month rats also did not differ on any of the behavioral measures. No significant differences were observed among the three groups on any of the anxiety measures (Figures 11 and 12). No differences were observed between the three groups on the number of entries made into the open arms, nor in the percentage of entries 
Figure 3. Percentage of time spent on the center hub. Pinealectomized rats spent significantly more time on the central hub compared to the other groups $(F(2,27)=3.29$, $\mathrm{p}<.05) . *$ represents differences between pinealectomized and sham groups. Error bars represent S.E.M. 


\section{Percentage Time on Center Hub}

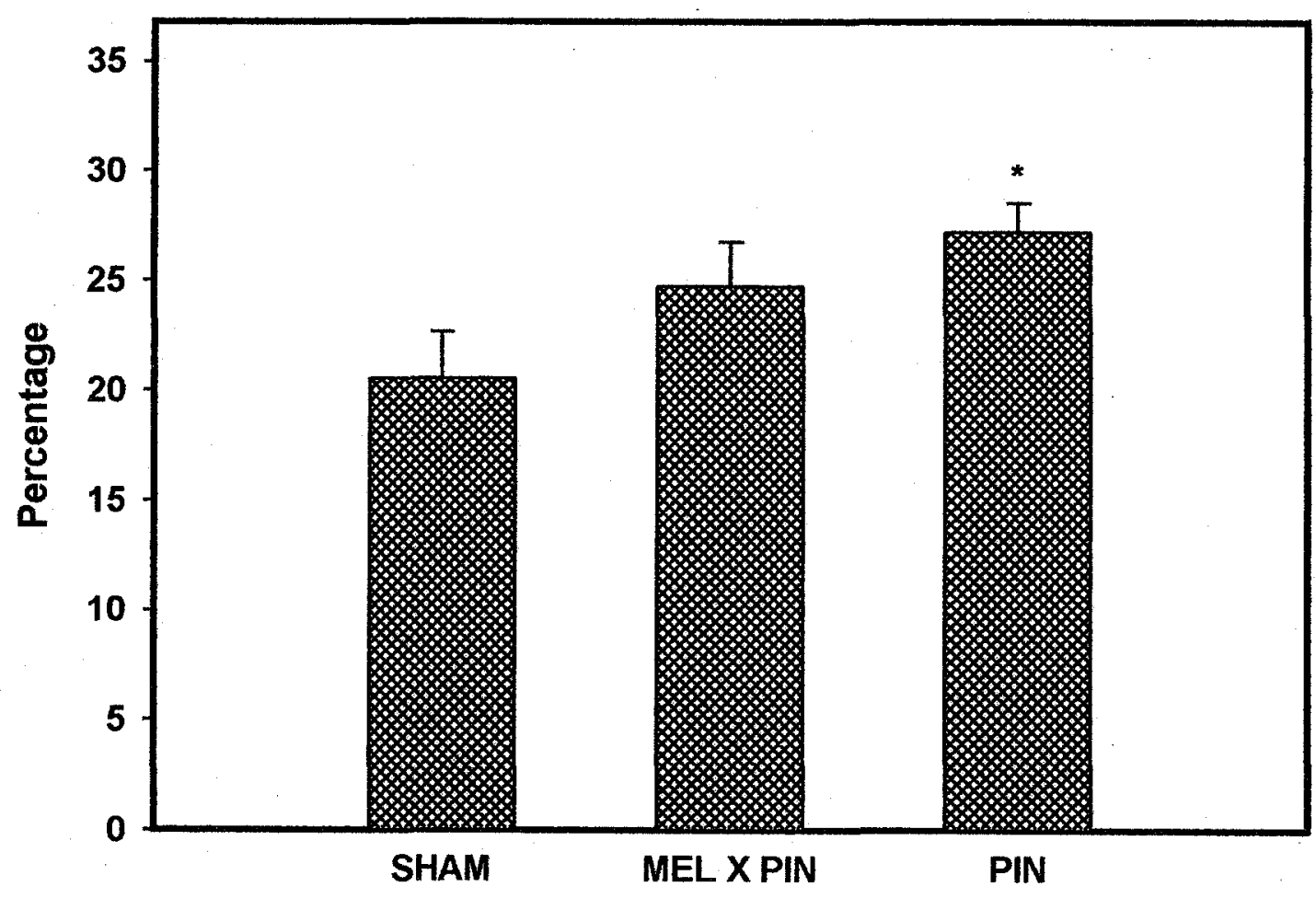


Figure 4. Top Panel: Total entries. At 2 months of age, the groups did not differ in the total number of arms that were entered. Error bars represent S.E.M. Bottom Panel: Closed arm entries. No significant group differences were found in the number of entries made into the closed arms. Error bars represent S.E.M. 
Total Entries

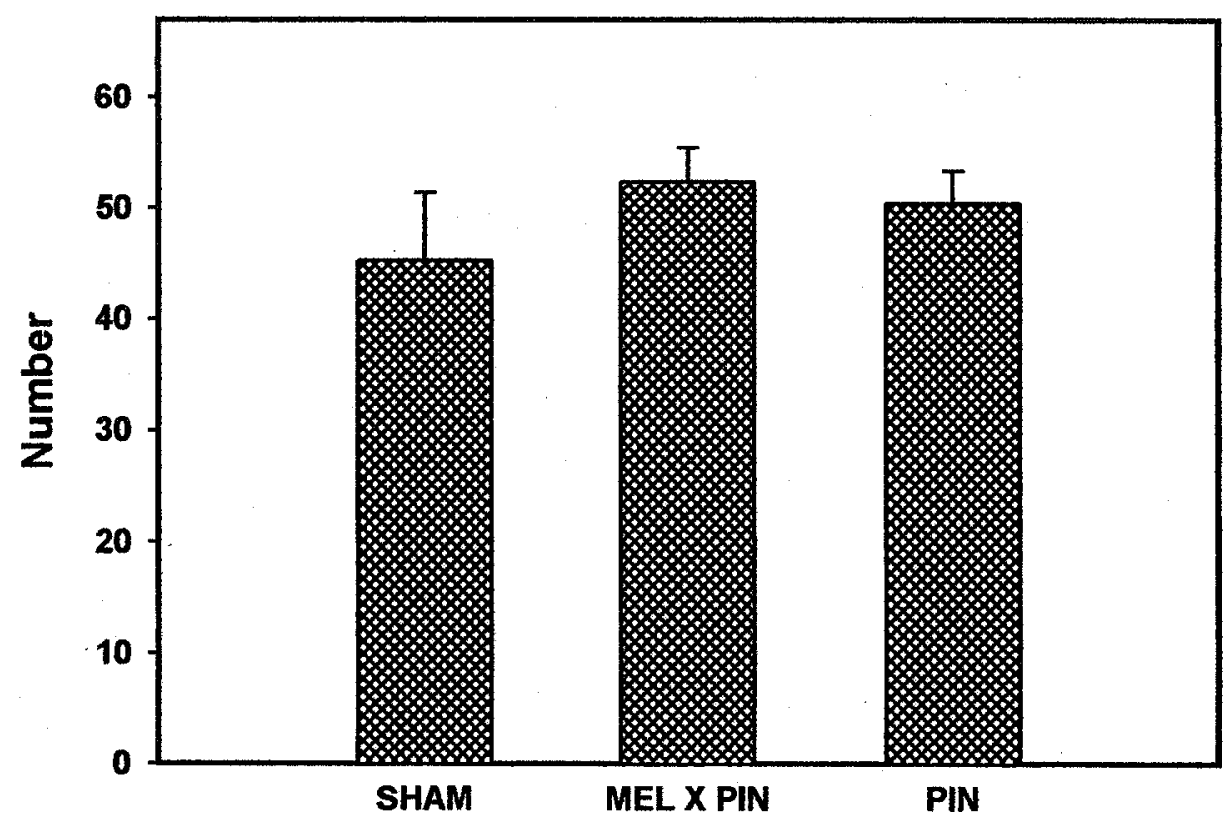

Closed Arm Entries

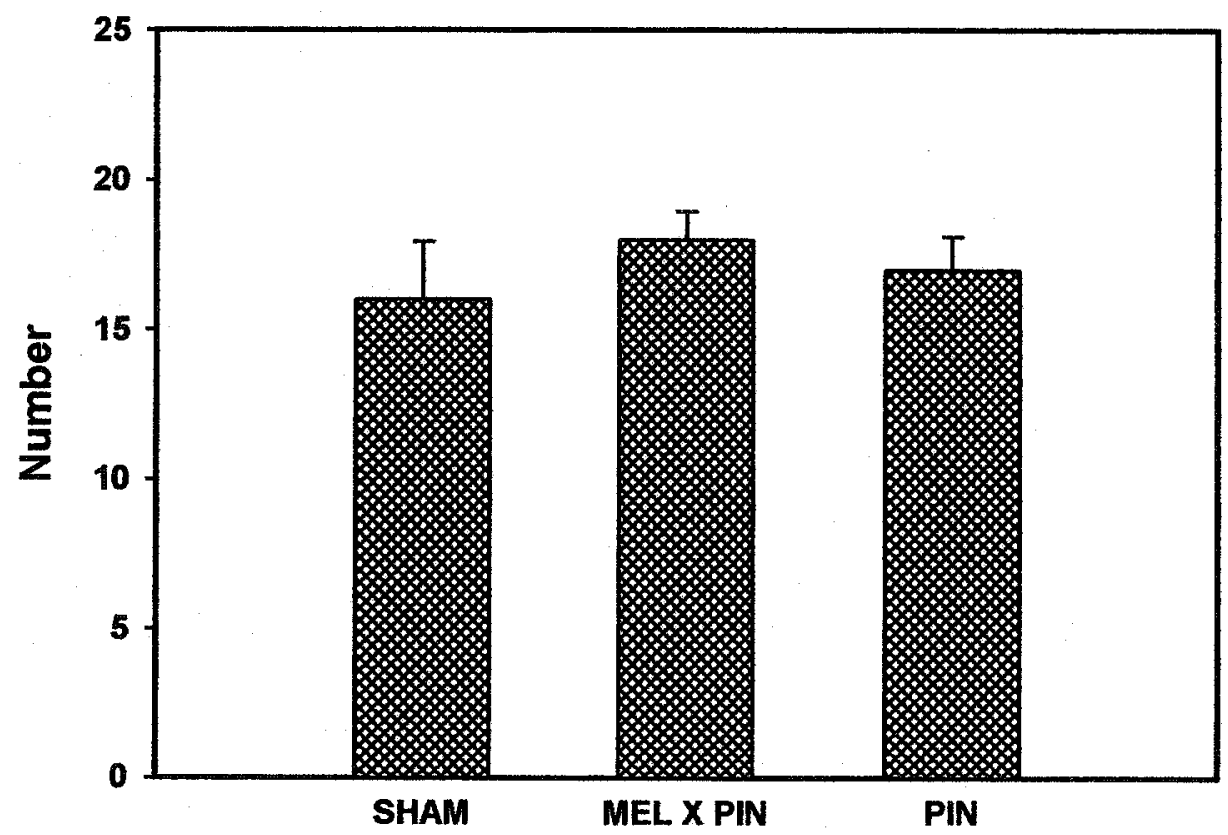


Figure 5. Top Panel: Number of head dips. At 2 months post-surgery, the groups did not differ on the number of head dips made in the open arms. Error bars represent S.E.M.

Bottom Panel: Number of vertical stretches. The groups did not differ on the number of vertical stretches made in the protected arms of the maze. Error bars represent S.E.M. 

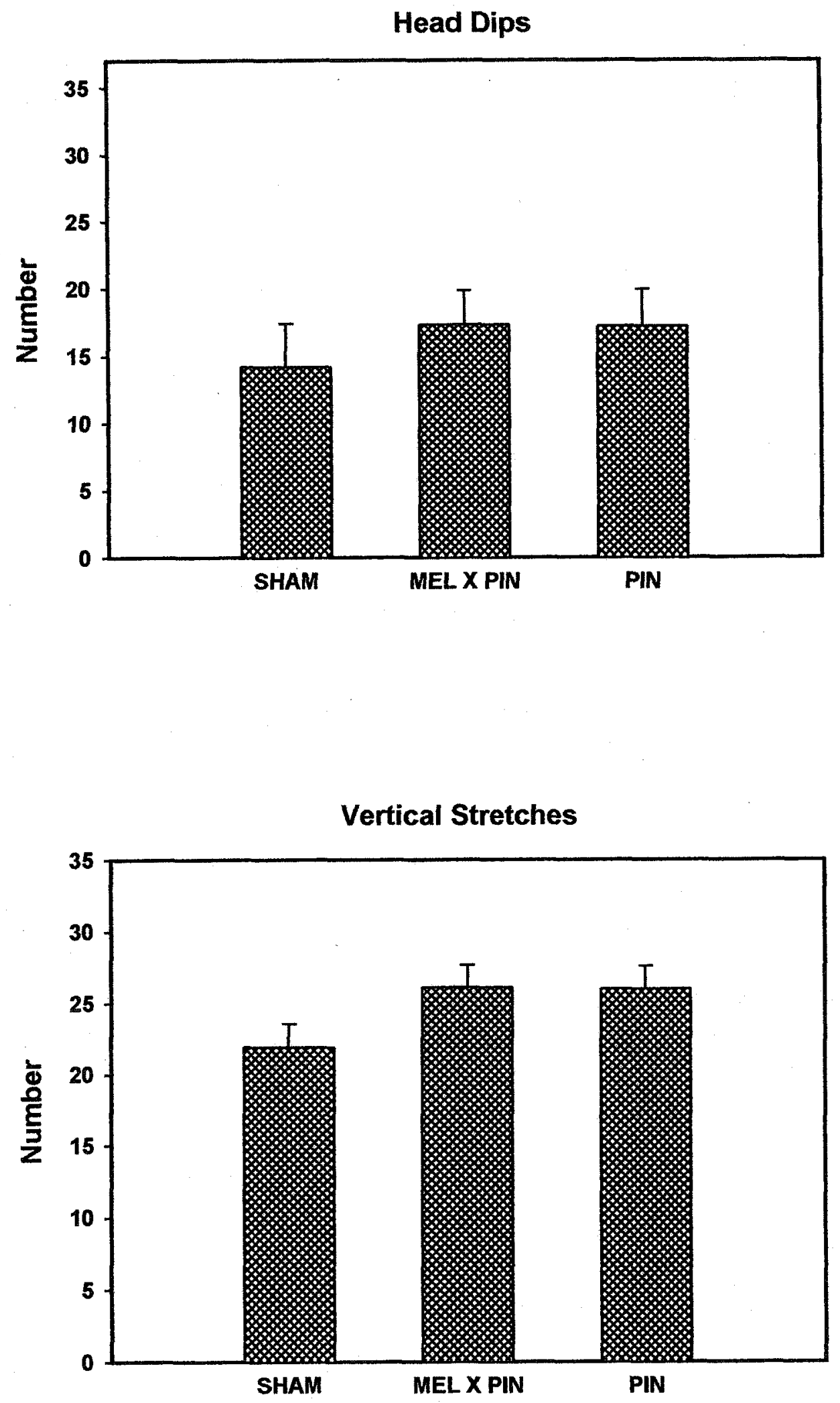
Figure 6. Top Panel: Number of open entries. At 7 months post-surgery, no significant differences were found between the groups on the number of open arms entered. Error bars represent S.E.M. Bottom Panel: Ratio of open arm entries. The groups did not differ in the percentage of entries made into the open arms. Error bars represent S.E.M. 

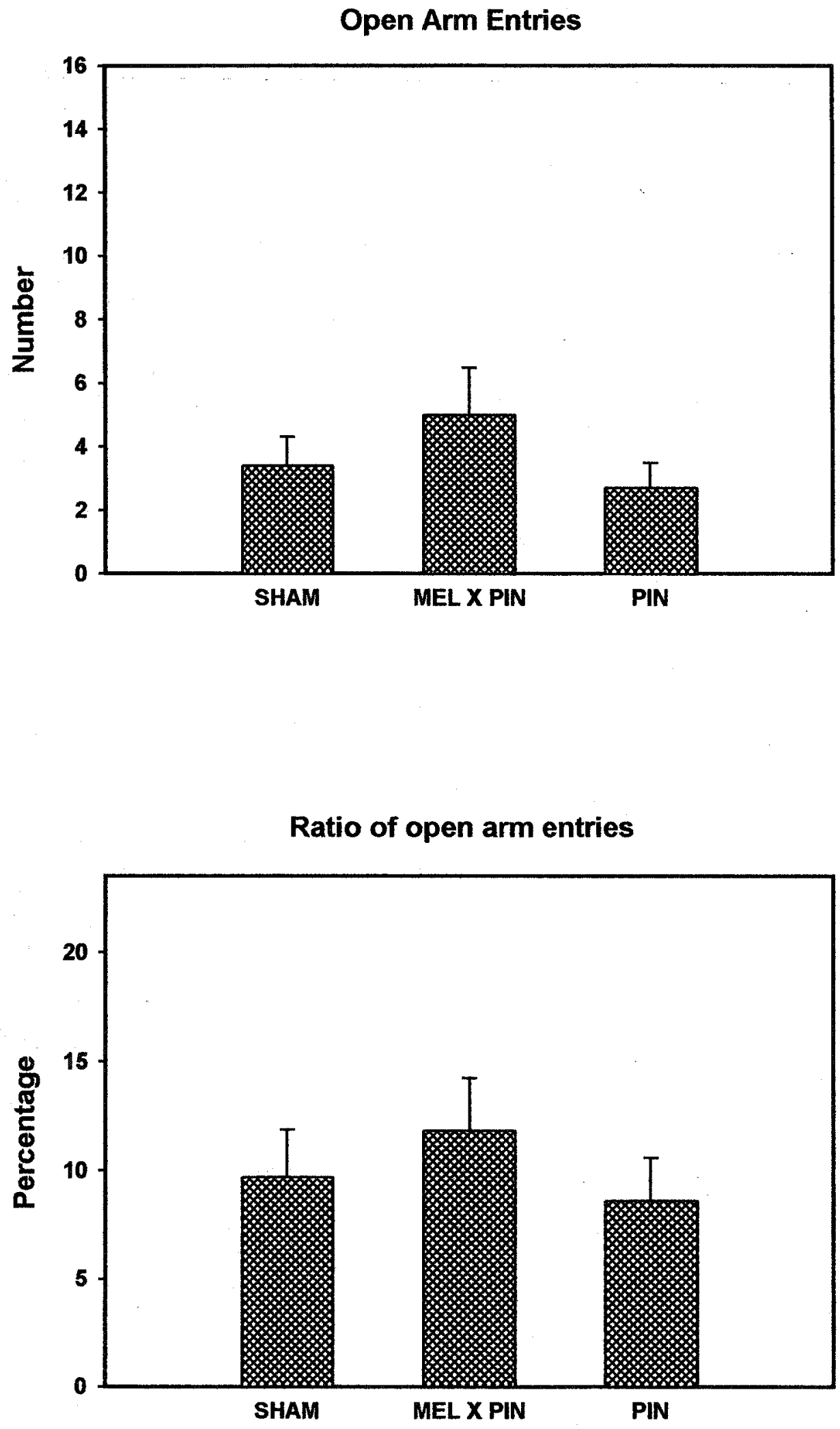
Figure 7. Top Panel: Ratio of time spent on the open arms. At 7 months of age, the groups did not differ significantly on the percentage of time spent in the open arms. Error bars represent S.E.M. Bottom Panel: Ratio of protected stretched attend postures. No significant differences were found between the groups on the percentage of stretch attend postures made from the closed arms. Error bars represent S.E.M. 
Ratio of Time Spent on Open arms

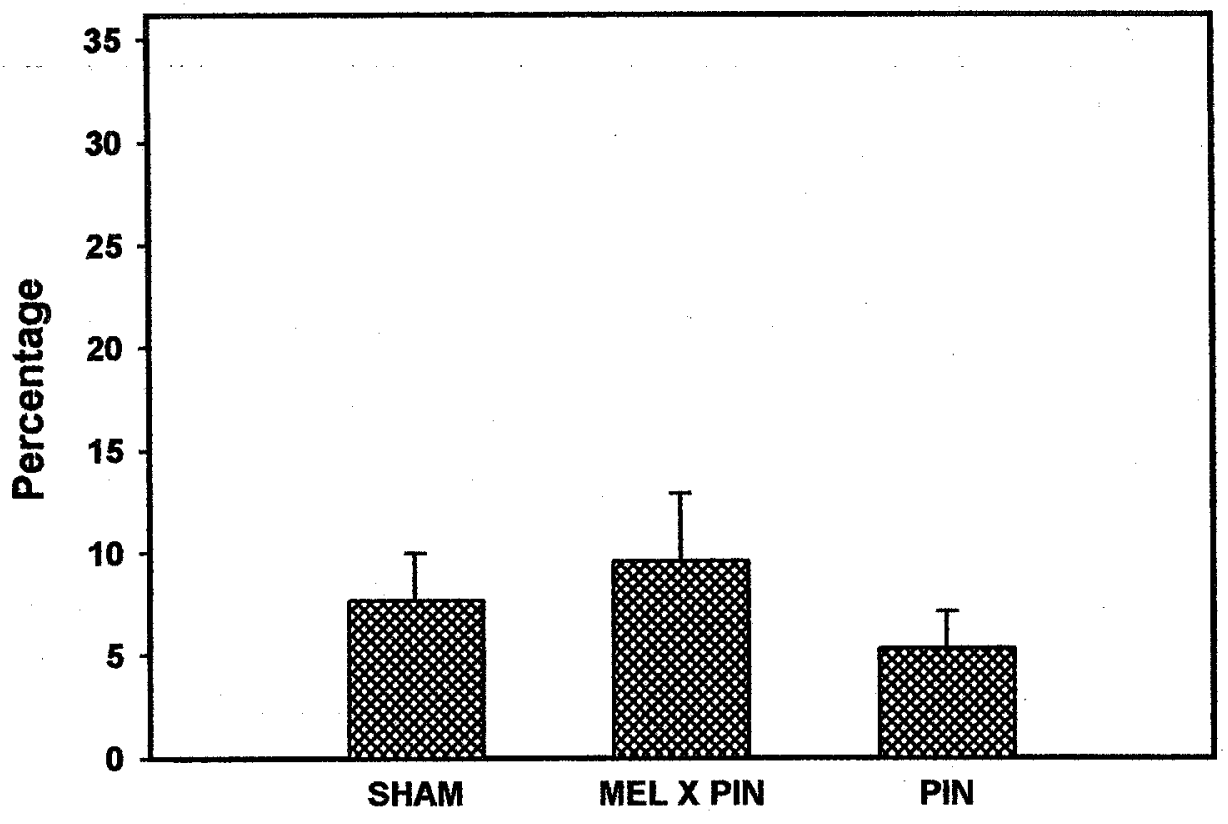

Ratio of Protected Stretched Attend Postures

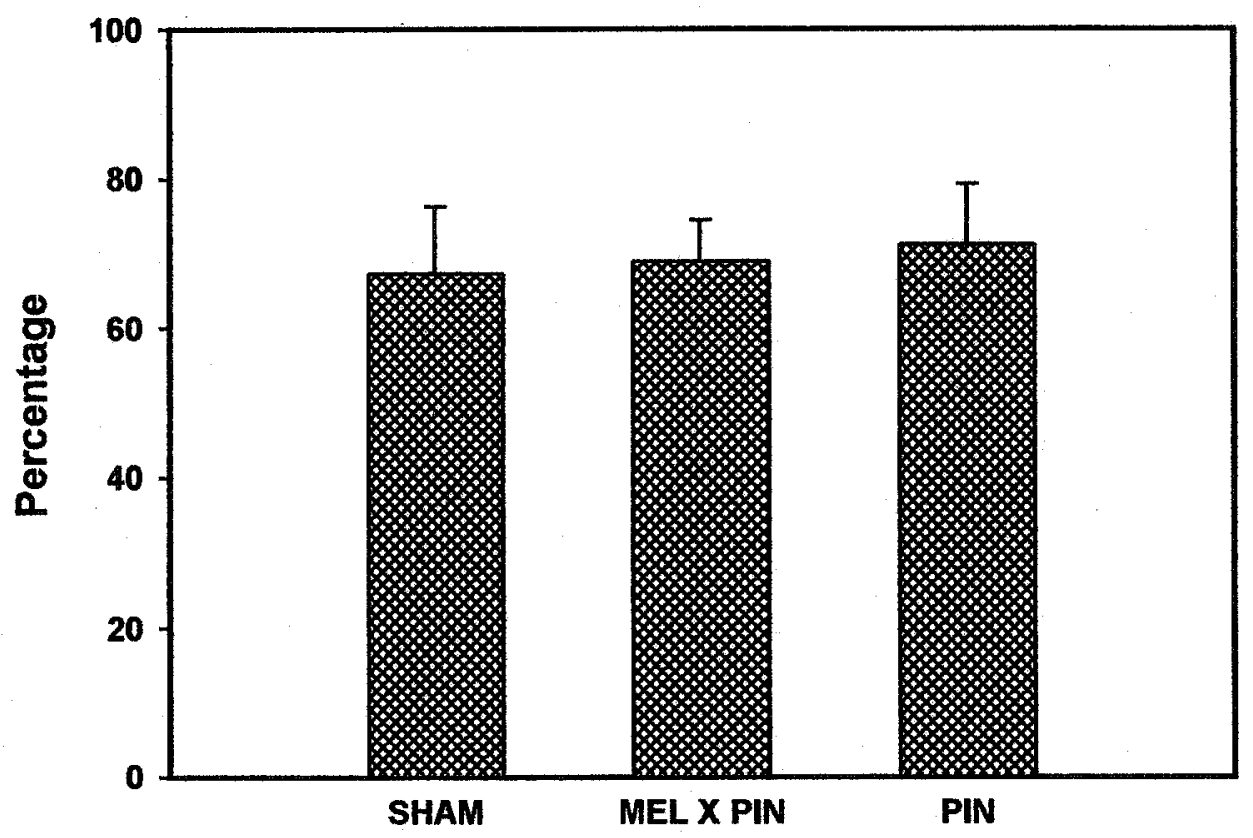


Figure 8. Ratio of time spent on the central hub. At 7 months post-surgery, the groups did not differ on the percentage of time spent in the central hub. Error bars represent S.E.M. 
Ratio of Time Spent in Center Hub

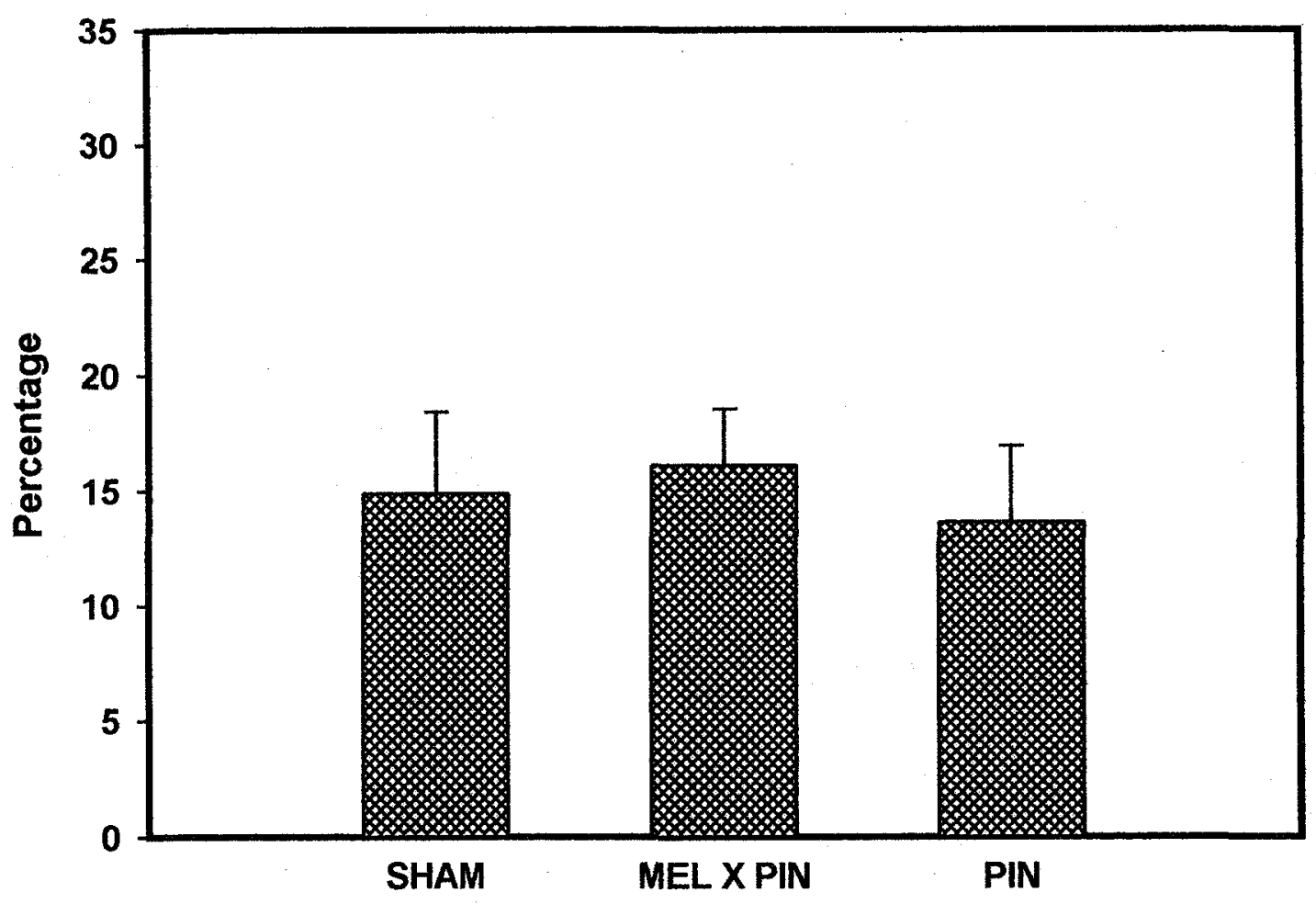


Figure 9. Top Panel: Number of total entries. No group differences were found in the number of total entries made on the maze. Error bars represent S.E.M. Bottom Panel: Closed arm entries. No significant group differences were found on the number of entries made into the closed arms of the maze. Error bars represent S.E.M. 
Total Entries

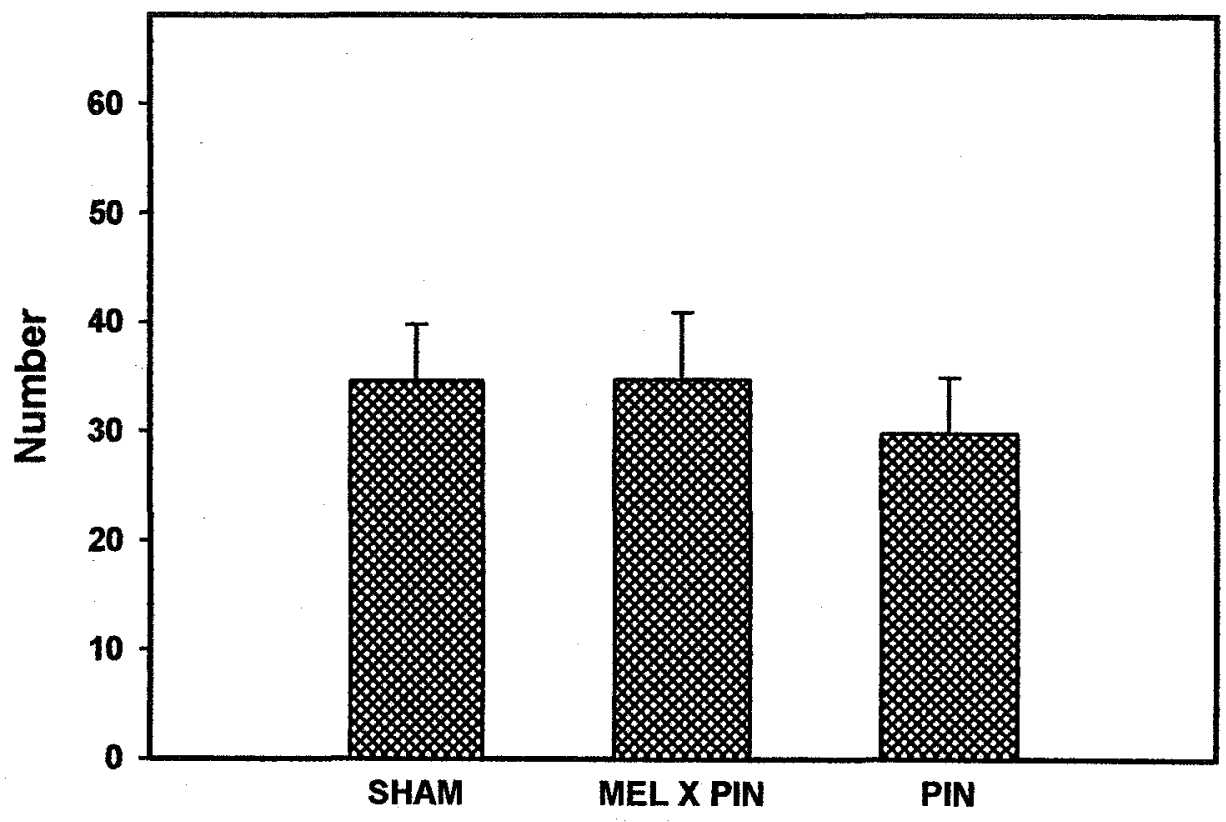

Closed Arm Entries

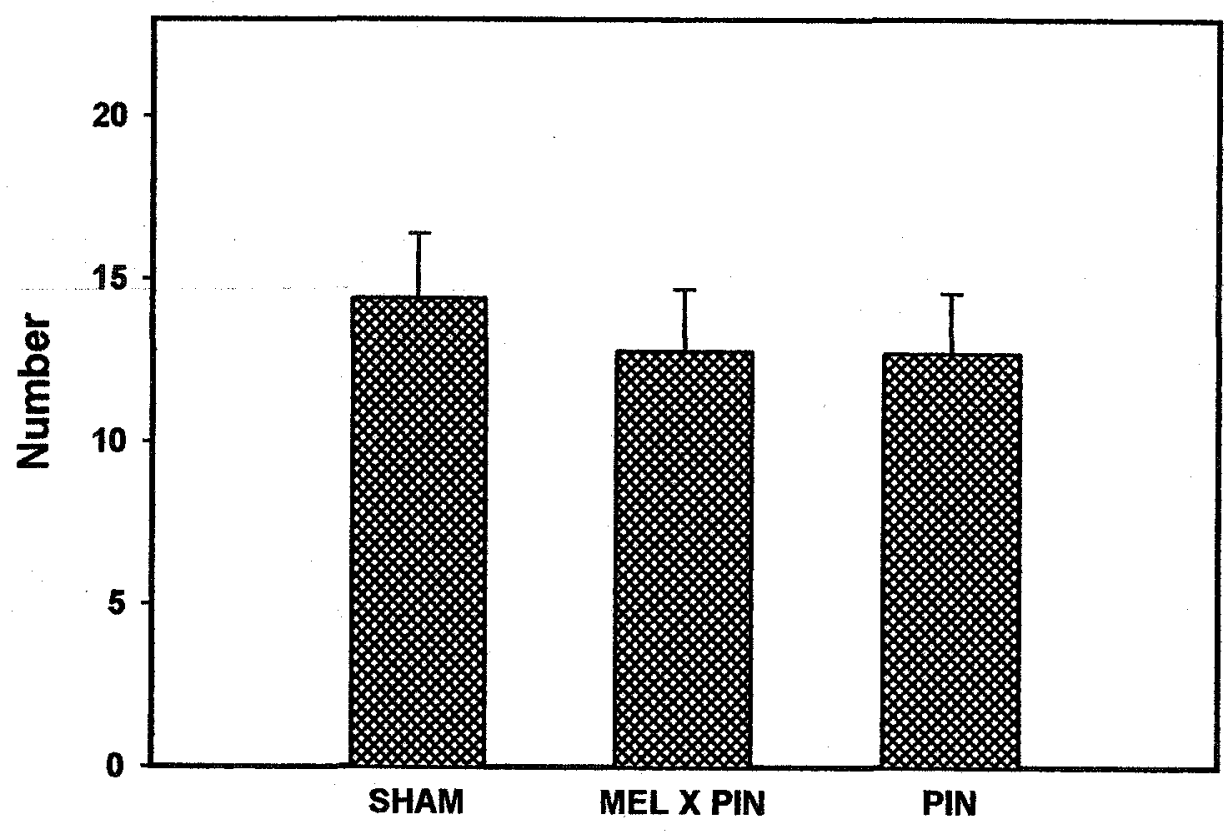


Figure 10. Top Panel: Number of head dips. The groups did not differ on the number of head dips made in the open arms. Error bars represent S.E.M. Bottom Panel: Number of vertical stretches. No significant group differences were found in the number of vertical stretches made in the closed arms. Error bars represent S.E.M. 

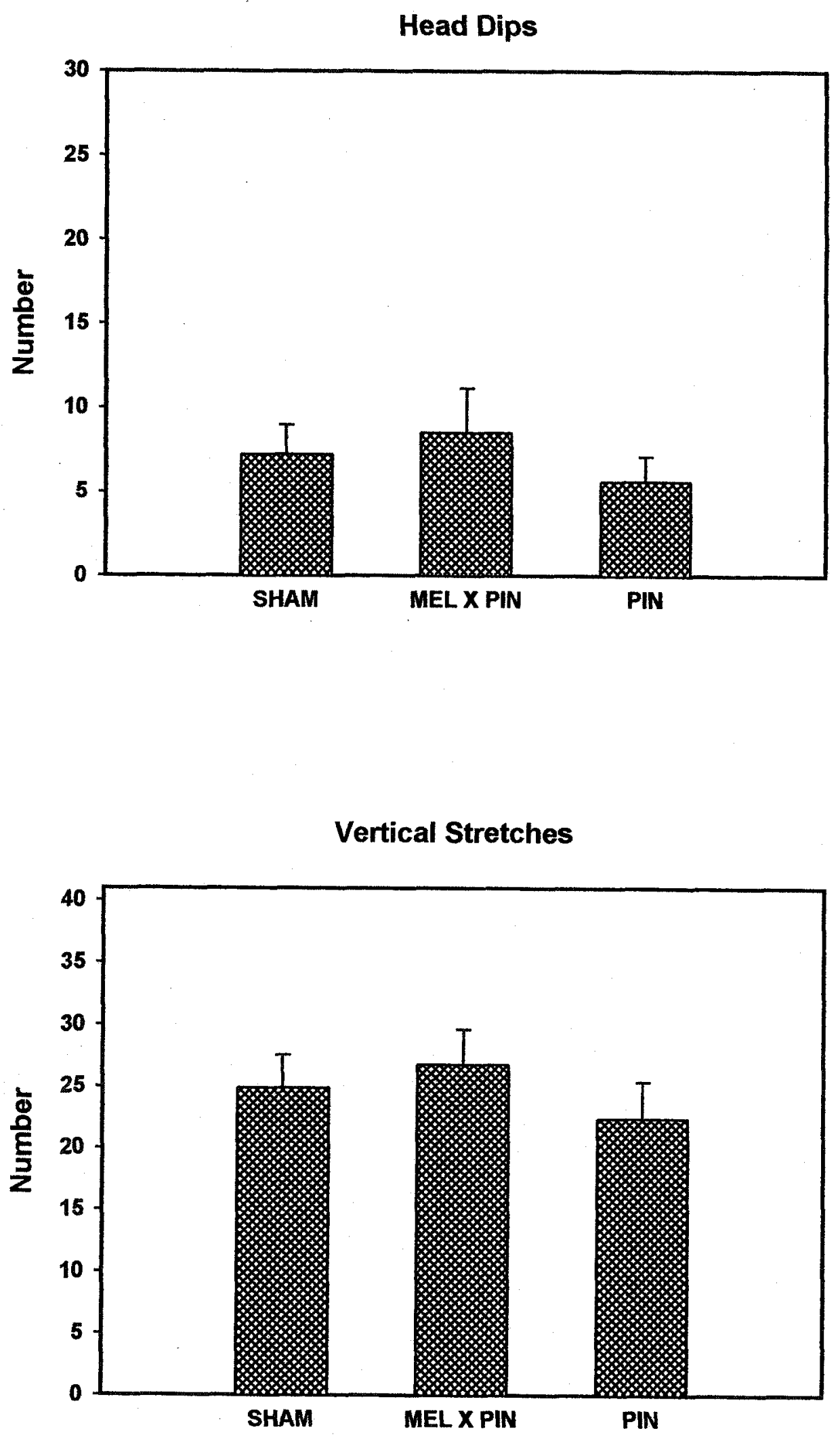
Figure 11. Top Panel: Number of open entries. At 11 -months of age, the groups did not differ in the number of entries into the open arms. Error bars represent S.E.M. Bottom Panel: Ratio of open arm entries. No group differences were found in the percentage of entries made into the open arms of the maze. Error bars represent S.E.M. 


\section{Open Arm Entries}

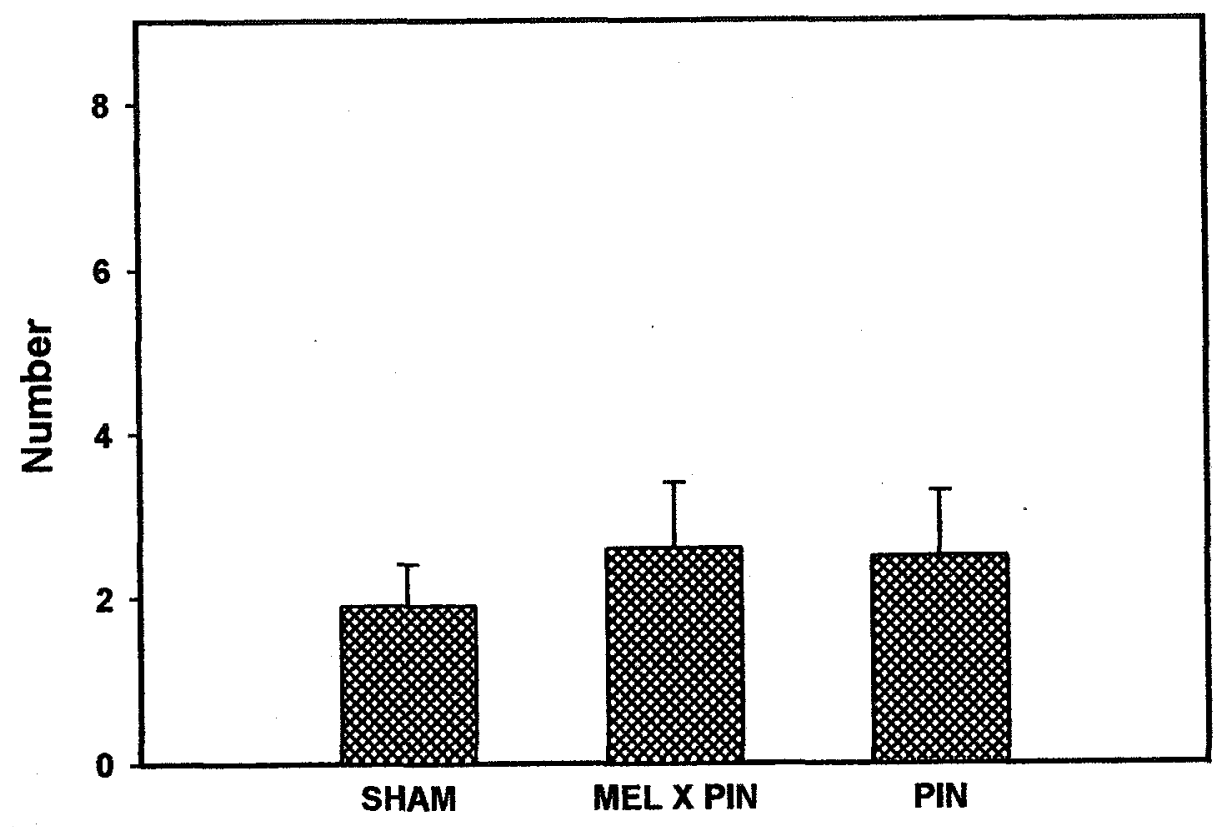

Percentage Entries in Open Arms

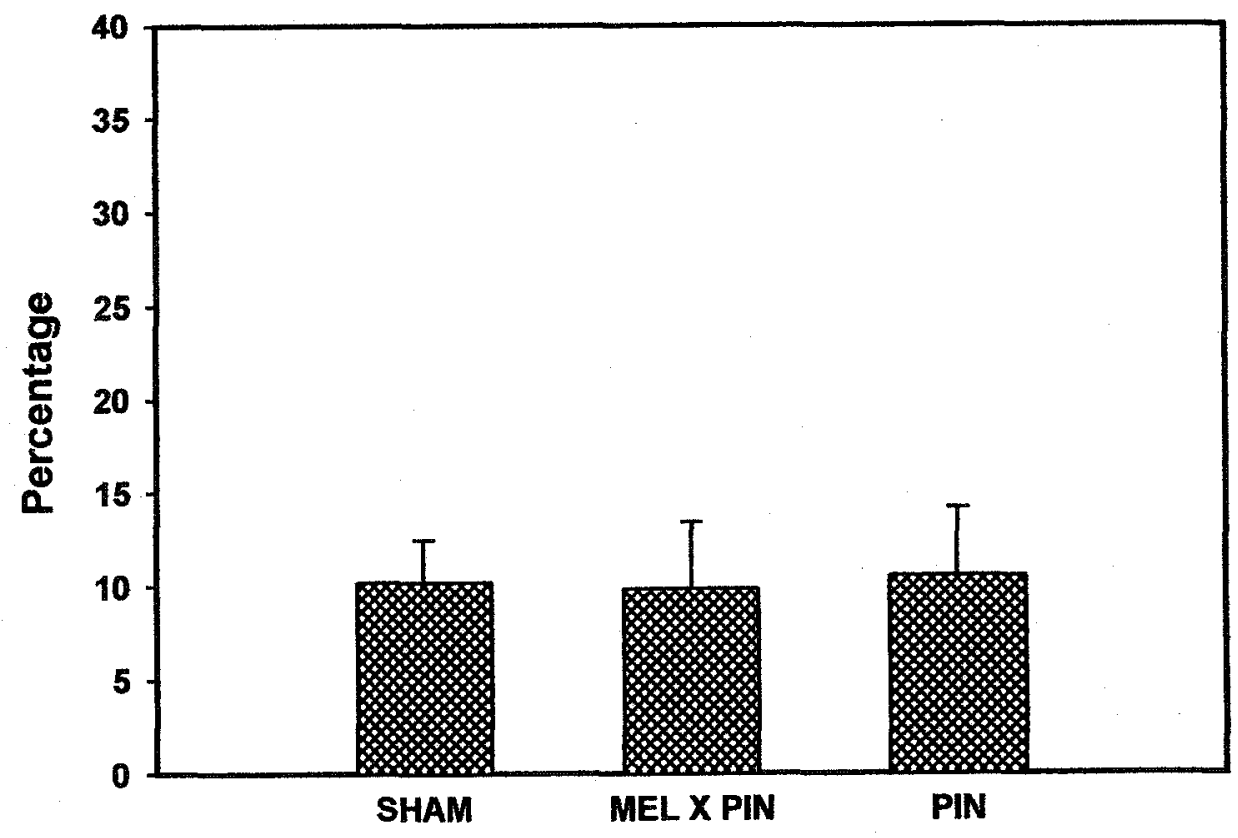


Figure 12. Top Panel: Ratio of time spent on the open arms. At 11-months post-surgery, the groups did not differ in the percentage of time spent in the open arms. Error bars represent S.E.M. Bottom Panel: Ratio of protected stretched attend postures. No group differences were found in the percentage of stretched attend postures made from the protected arms. Error bars represent S.E.M. 
Ratio of Time Spent in Open Arms

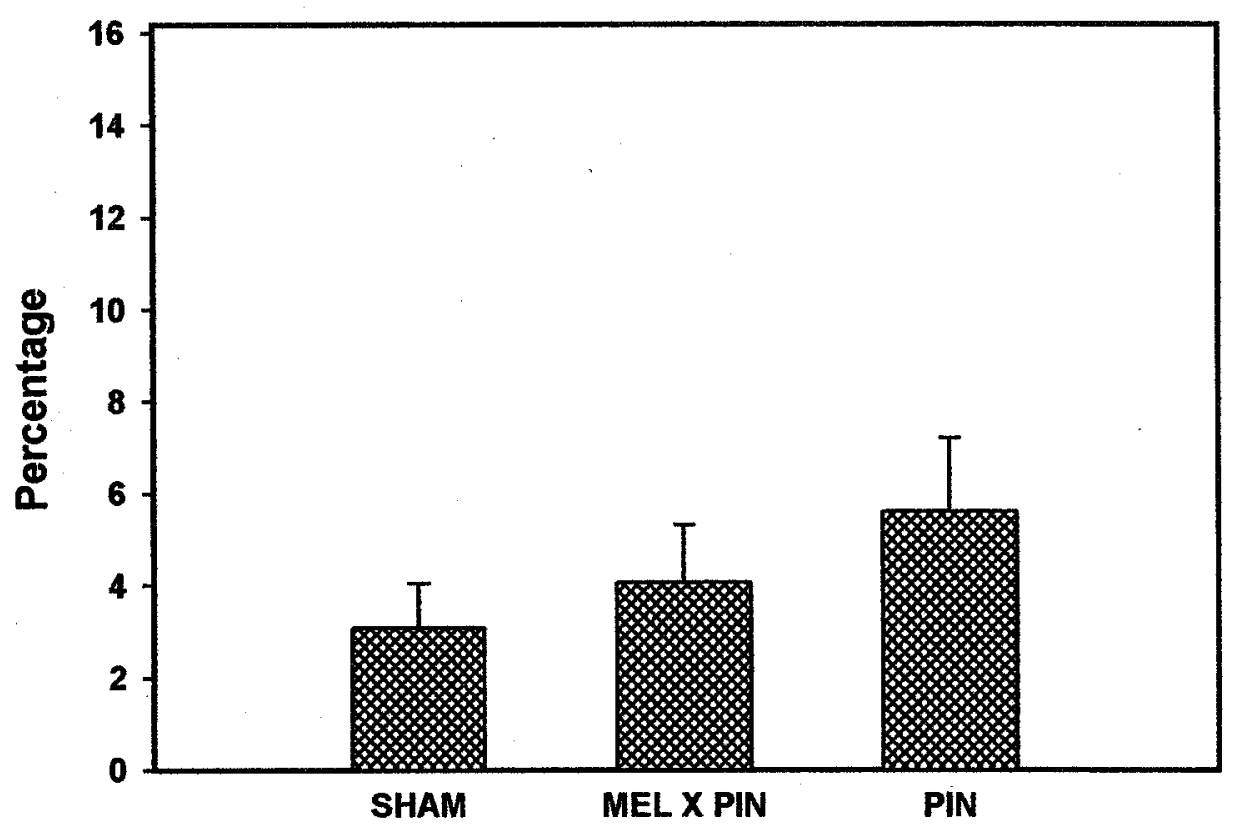

Ratio of Protected Stretched Attend Postures

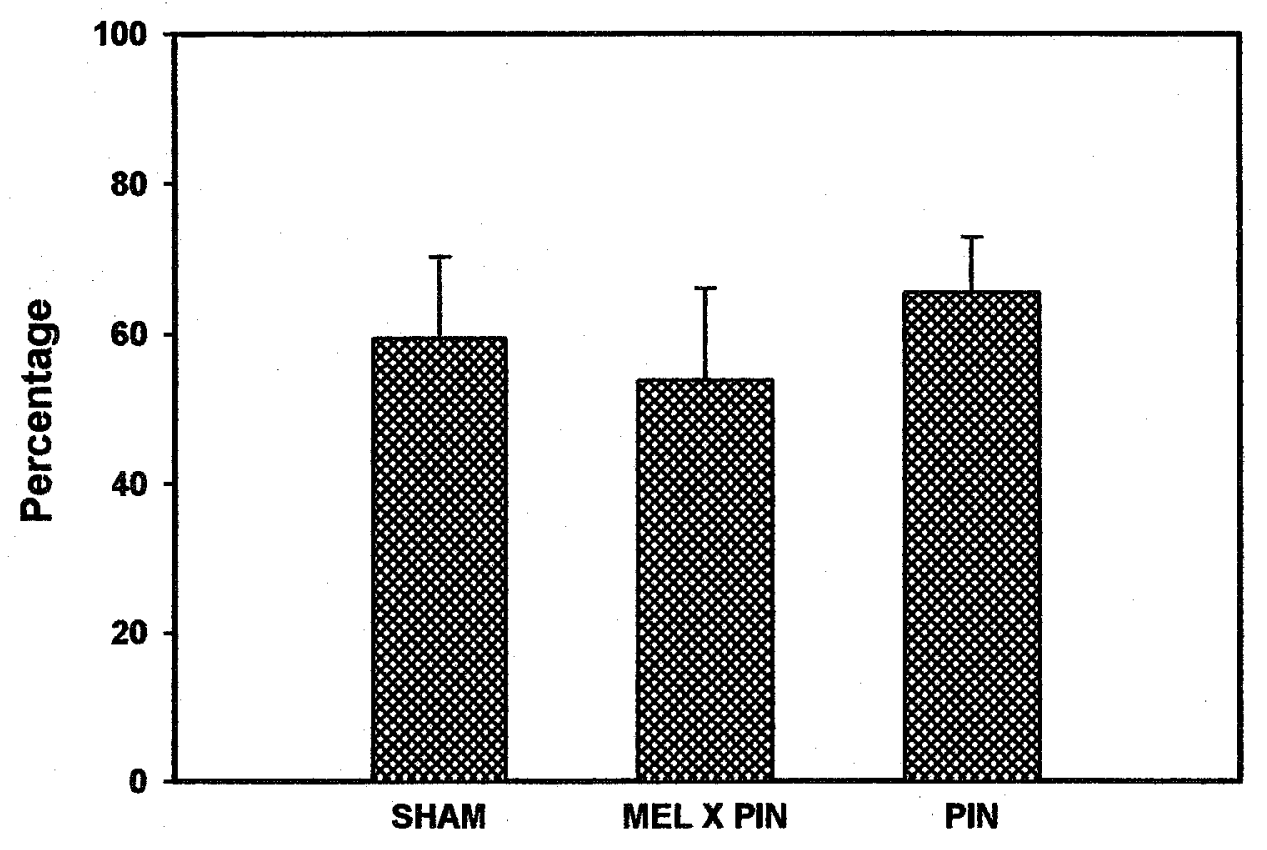


made into the open arms. Moreover, the groups did not differ on the percentage of time that was spent in the open arms. Furthermore, the proportion of protected stretch attend postures observed in the closed arms did not differ between the groups. No significant difference was observed between the three groups on the decision making index (Figure 13). In addition, all groups exhibited similar locomotor and exploratory behaviors on the maze (Figures 14 and 15). Specifically, the groups did not differ on the total number of arms entered or on the number of closed arm entries. The groups also did not differ on the number of head dips made in the closed arms, or on the number of vertical stretches made in the closed arms.

At 15-months, no significant difference was observed between the groups on any of the anxiety measures (Figures 16 and 17). No difference was found between the groups in the number of entries into the open arms nor in the percentage of entries made into the open arms. Furthermore, no group differences were found in the percentage of time spent on the open arms nor in the percentage of protected stretched attend postures made in the closed arms. Moreover, no significant group difference was seen in the percentage of time spent in the central hub, a decision index (Figure 18).

There was a significant group difference in locomotor activity on the maze $(F(2,27)=3.33, p<.05)$ (Figure 19). Melatonin supplemented animals entered significantly more arms than the sham animals. The group comparison for entries made into the closed arms, narrowly missed reaching significance $(p=.06)$ (Figure 19). Although not significant, melatonin supplemented animals made more closed arm entries. All three groups exhibited similar exploratory behaviors on the elevated plus maze (Figures 20). 
Figure 13. Ratio of time spent on the central hub. At 11 months post-surgery, the groups did not differ in the amount of time spent in the central hub of the maze. Error bars represent S.E.M. 
Ratio of Time Spent in Center Hub

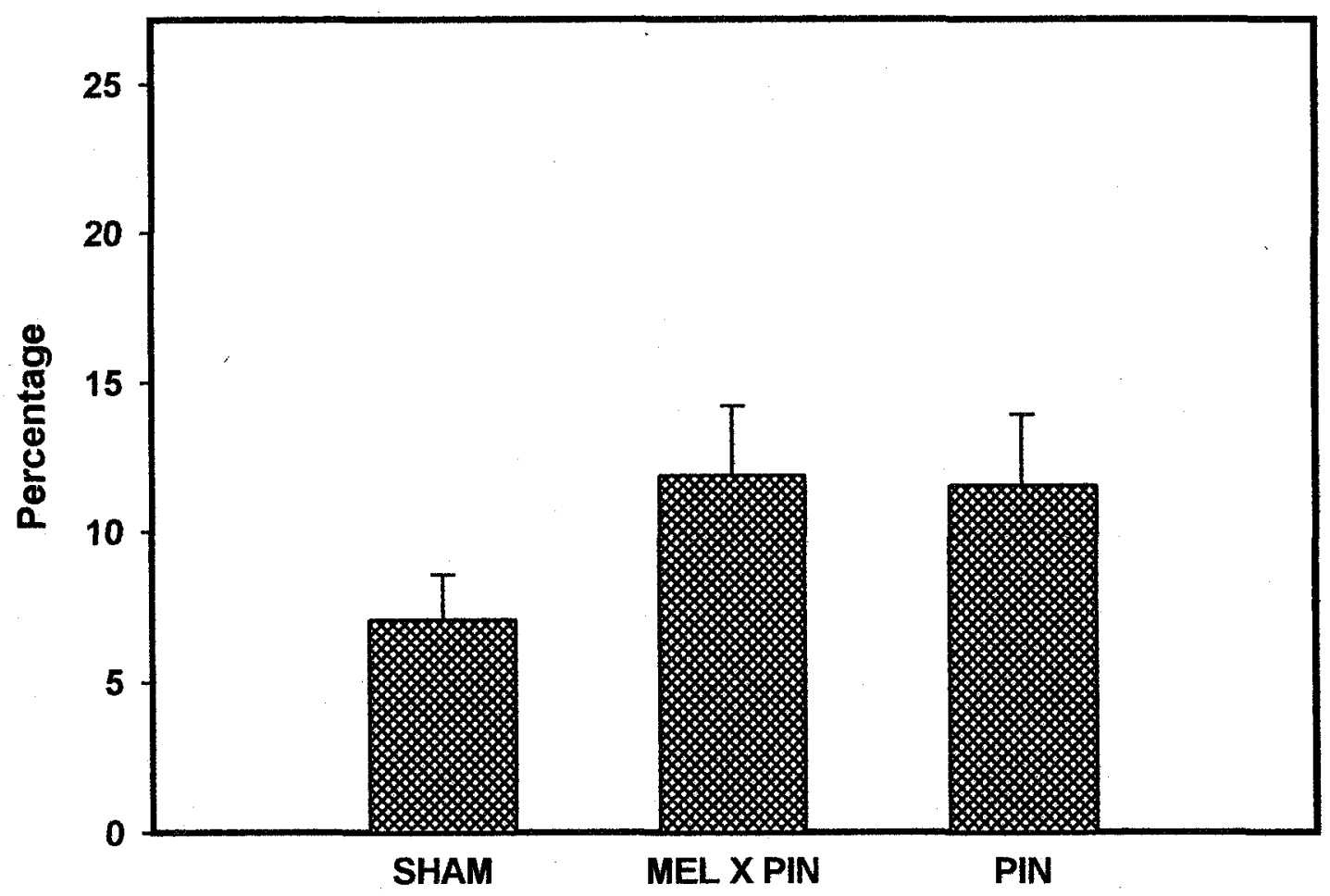


Figure 14. Top Panel: Total Entries. At 11 months of age, the groups did not differ in the number of total entries made on the maze. Error bars represent S.E.M. Bottom Panel: Closed arm entries. No significant group differences were found in the number of closed arms entered on the maze. Error bars represent S.E.M. 
Total Entries

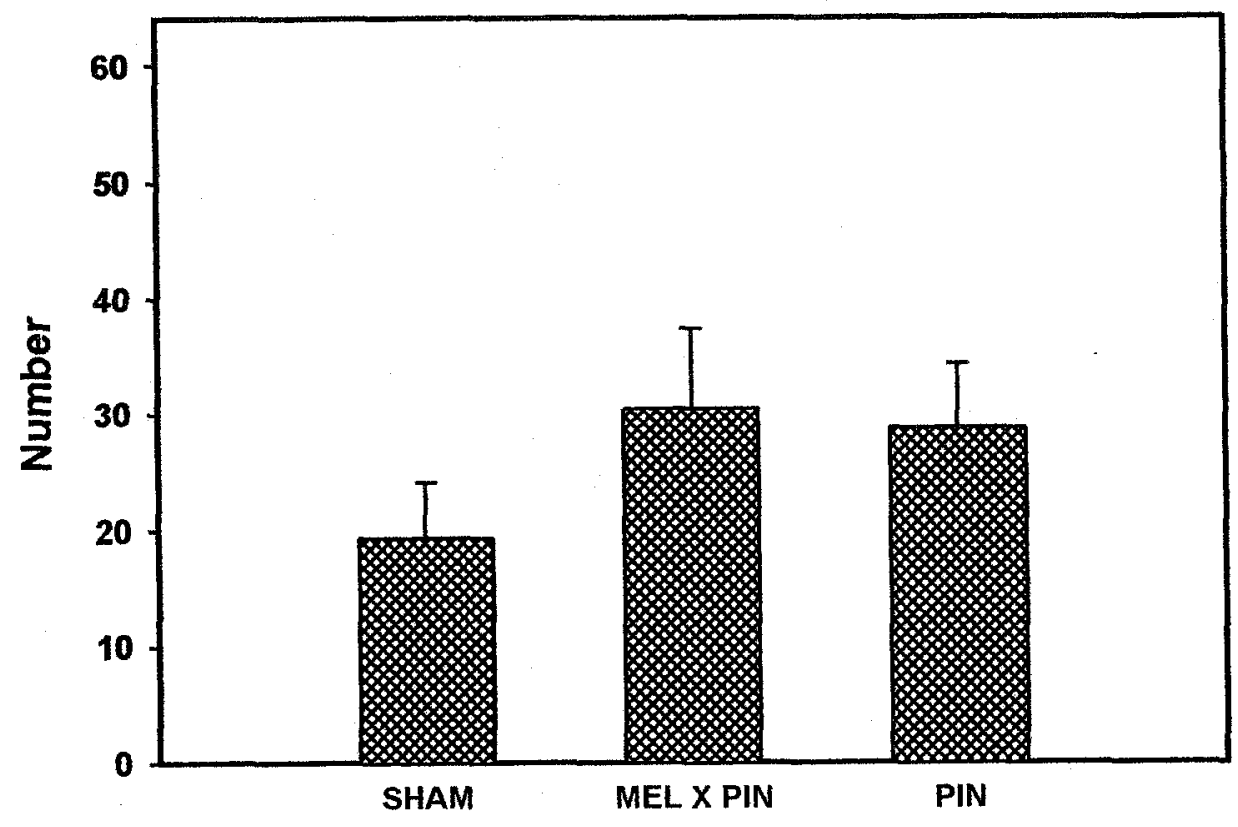

Closed Arm Entries

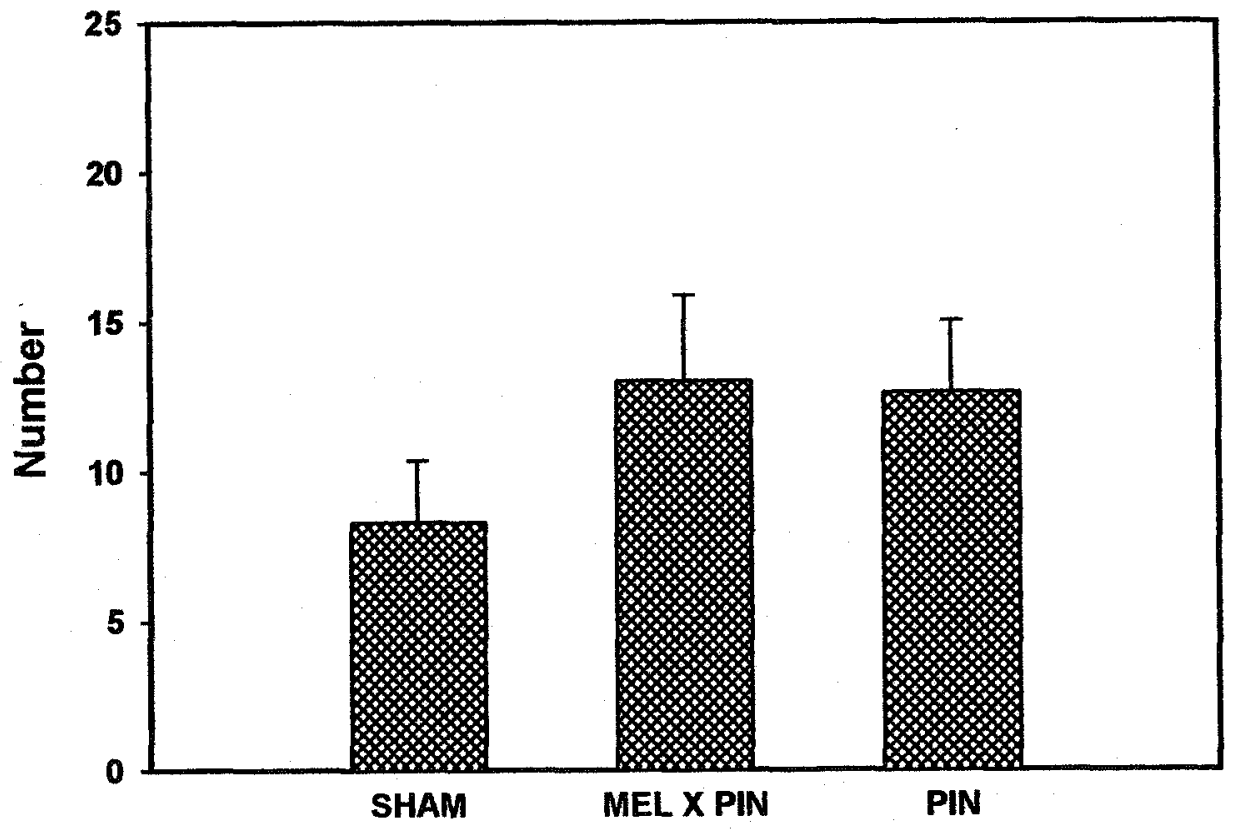


Figure 15. Top Panel: Number of head dips. At 11-months post-surgery, the groups did not differ in the number of head dips made in the open arms. Error bars represent S.E.M. Bottom Panel: Number of vertical stretches. No group differences were made in the number of vertical stretches made in the closed arms. Error bars represent S.E.M. 

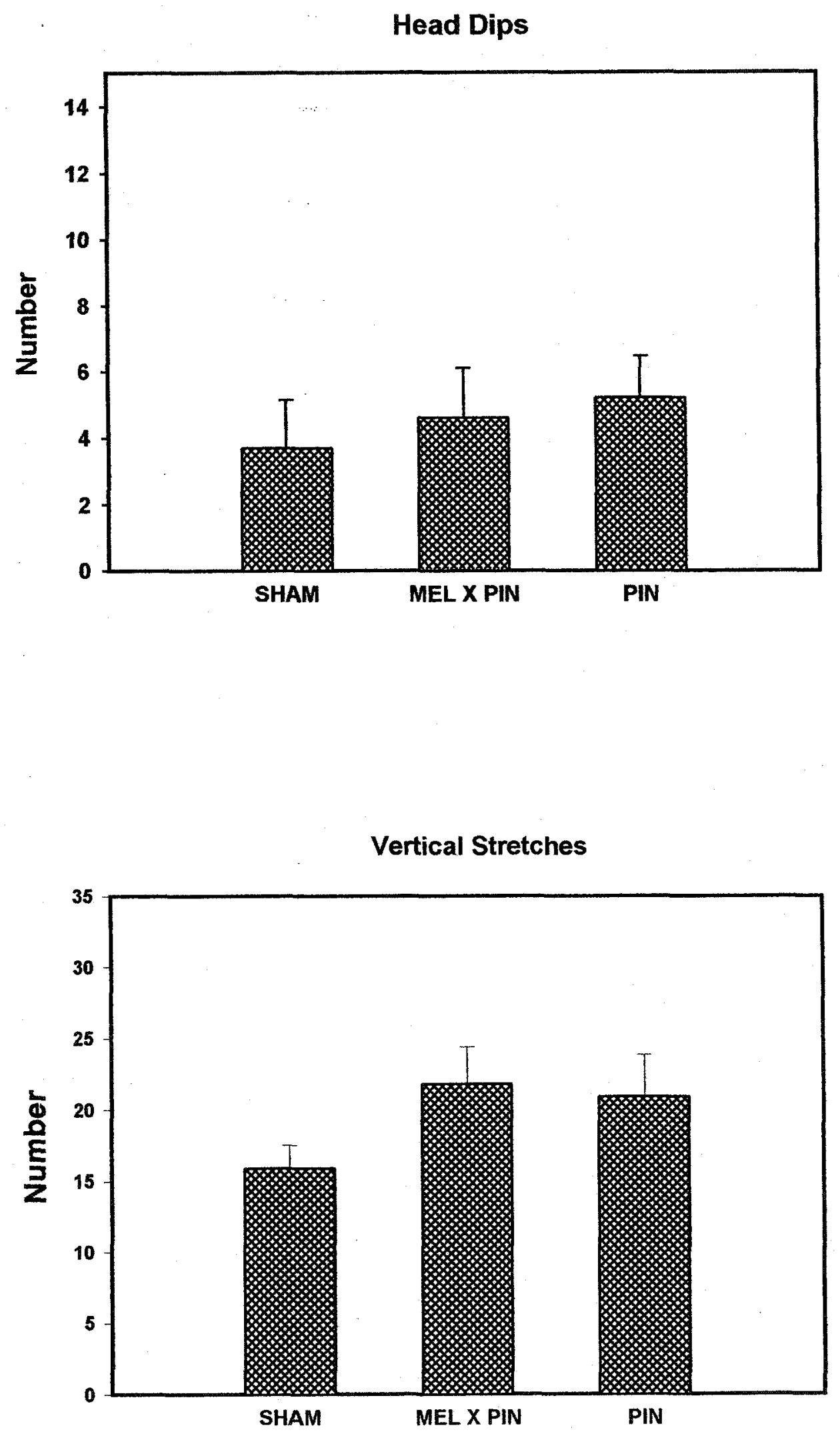
Figure 16. Top Panel: Number of open entries. At 15 months post-surgery, the groups did not differ on the number of entries made into the open arms. Error bars represent S.E.M. Bottom Panel: Ratio of open entries. No group differences were found in the percentage of entries made into the open arms. Error bars represent S.E.M. 


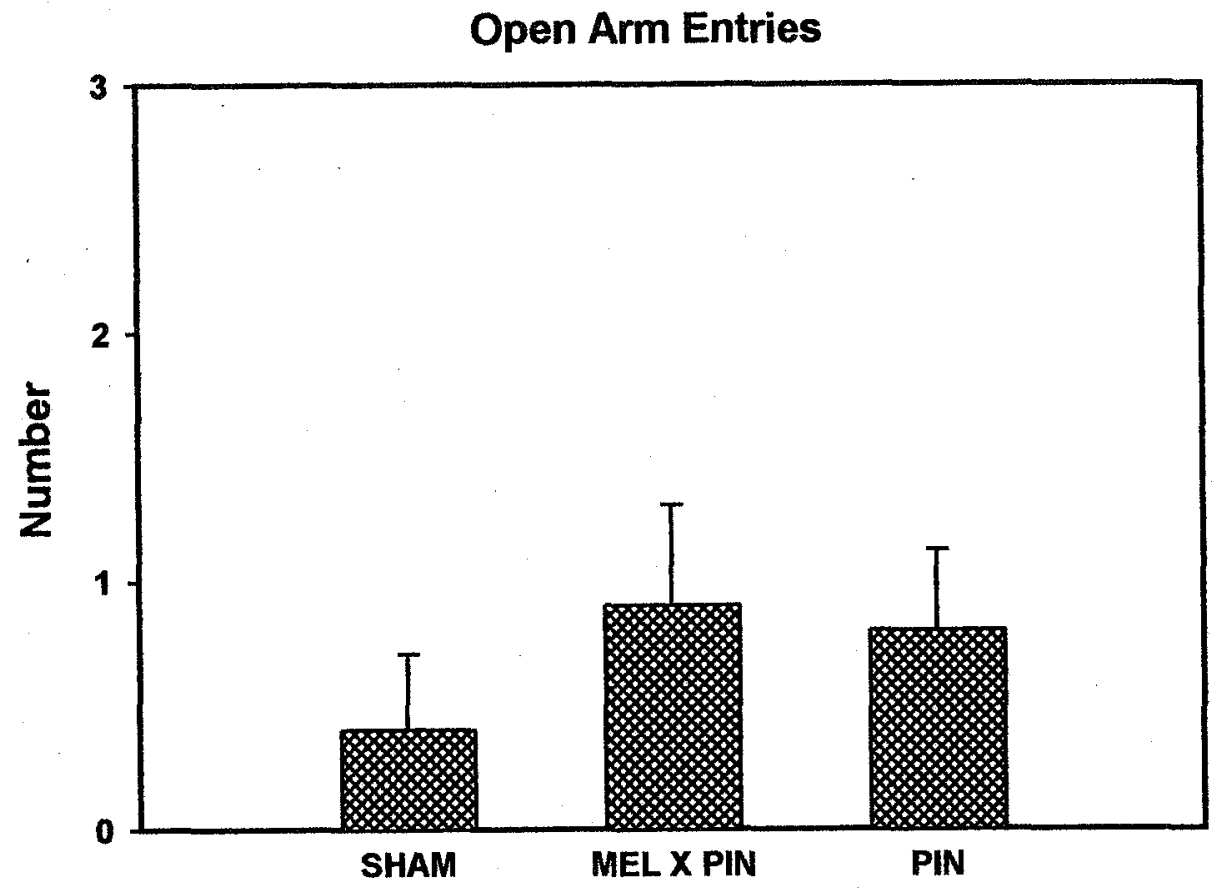

Ratio of Entries in Open Arms

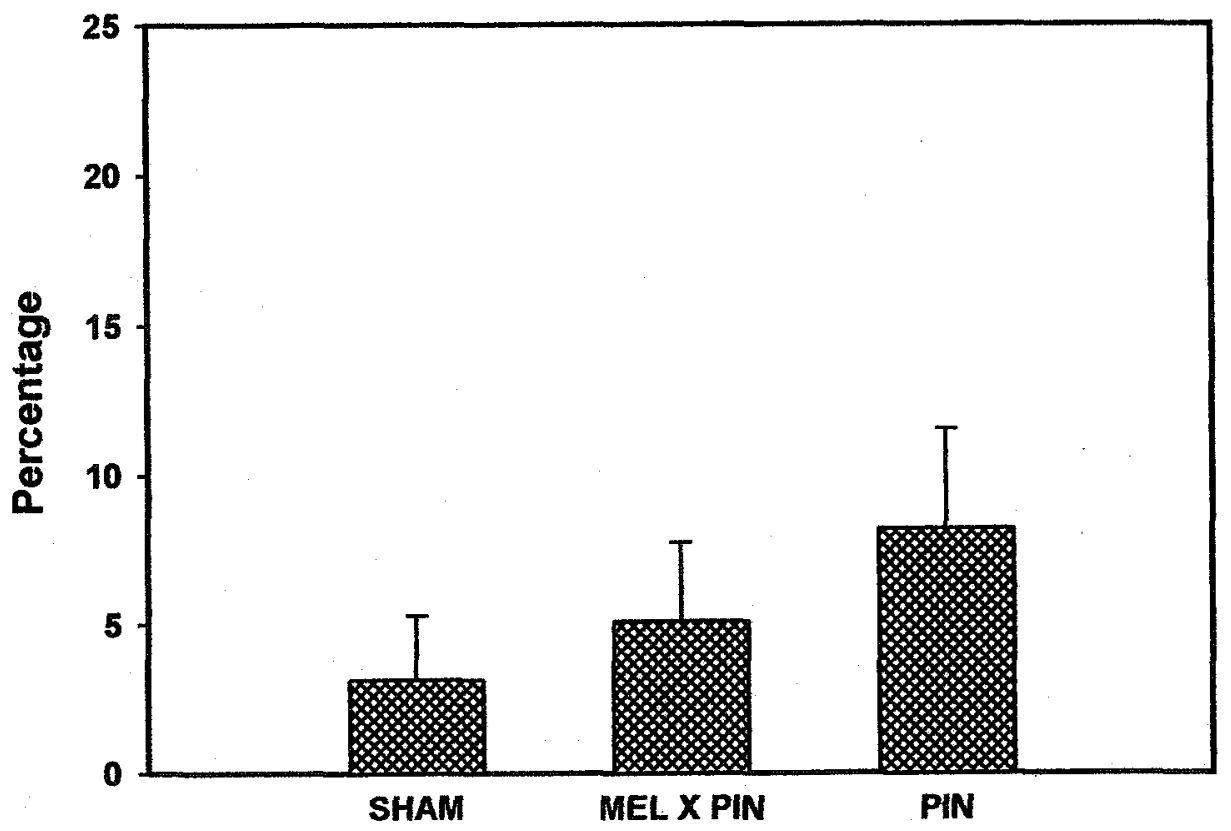


Figure 17. Top Panel: Ratio of time spent on open arms. At 15-months post-surgery, the groups did not differ in the amount of time spent in the unprotected open arms. Error bars represent S.E.M. Bottom Panel: Ratio of protected stretched attend postures. The groups did not differ in the percentage of stretch attend postures made from the protected arms of the maze. Error bars represent S.E.M. 

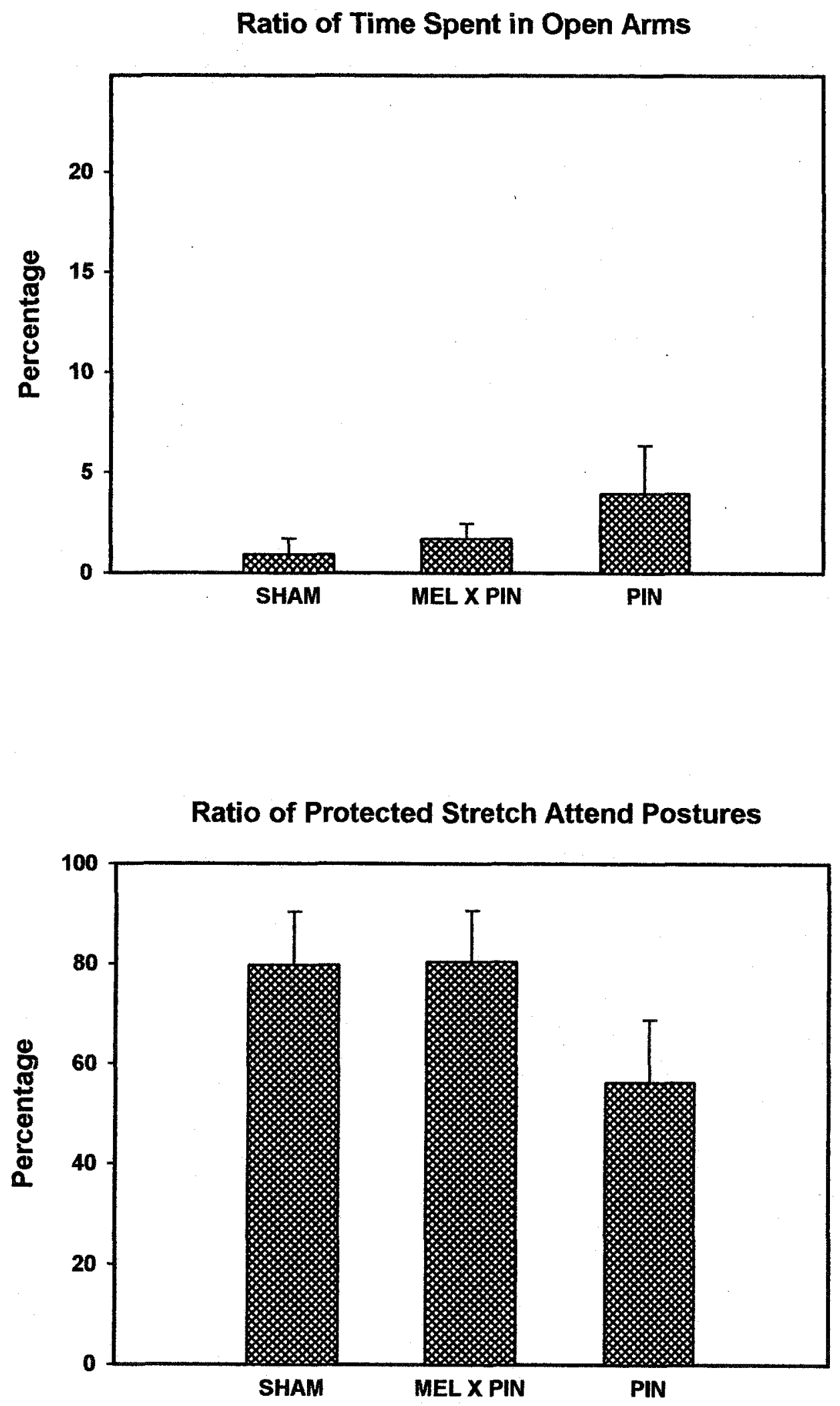
Figure 18. Ratio of time spent in central hub. At 15-months of age, the groups did not differ in the amount of time spent in the central hub of the maze. Error bars represent S.E.M. 
Ratio of Time Spent in Center Hub

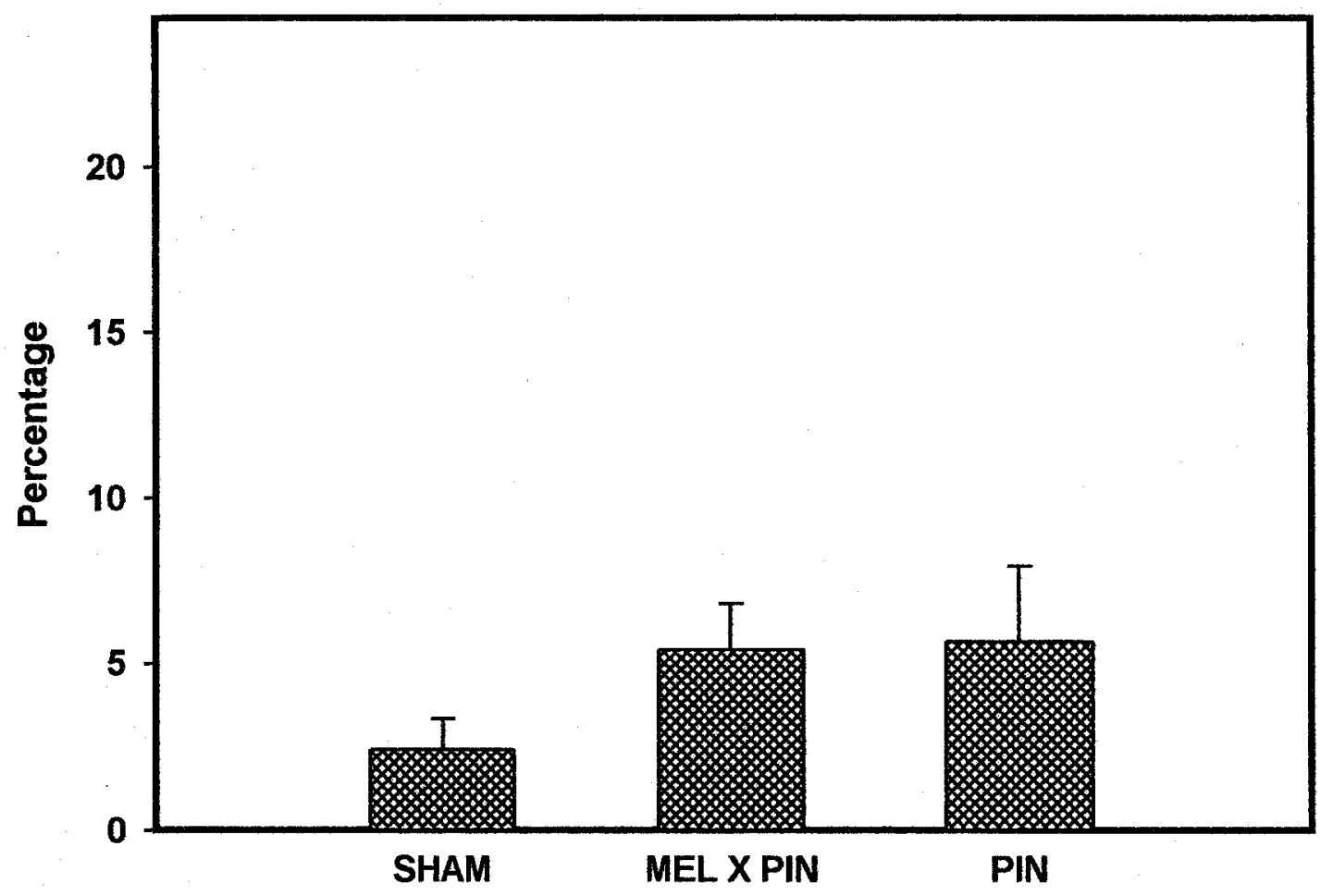


Figure 19. Top Panel: Total entries. At 15 months post-surgery, melatonin supplemented rats made significantly more arm entries compared to the sham rats $(\mathrm{F}(2$, $27)=3.33, \mathrm{p}<.05){ }^{*}$ represents differences between melatonin supplemented and sham groups. Error bars represent S.E.M. Bottom Panel: Closed arm entries. No group differences were found in the number of entries into the closed arms. Error bars represent S.E.M 
Total Entries

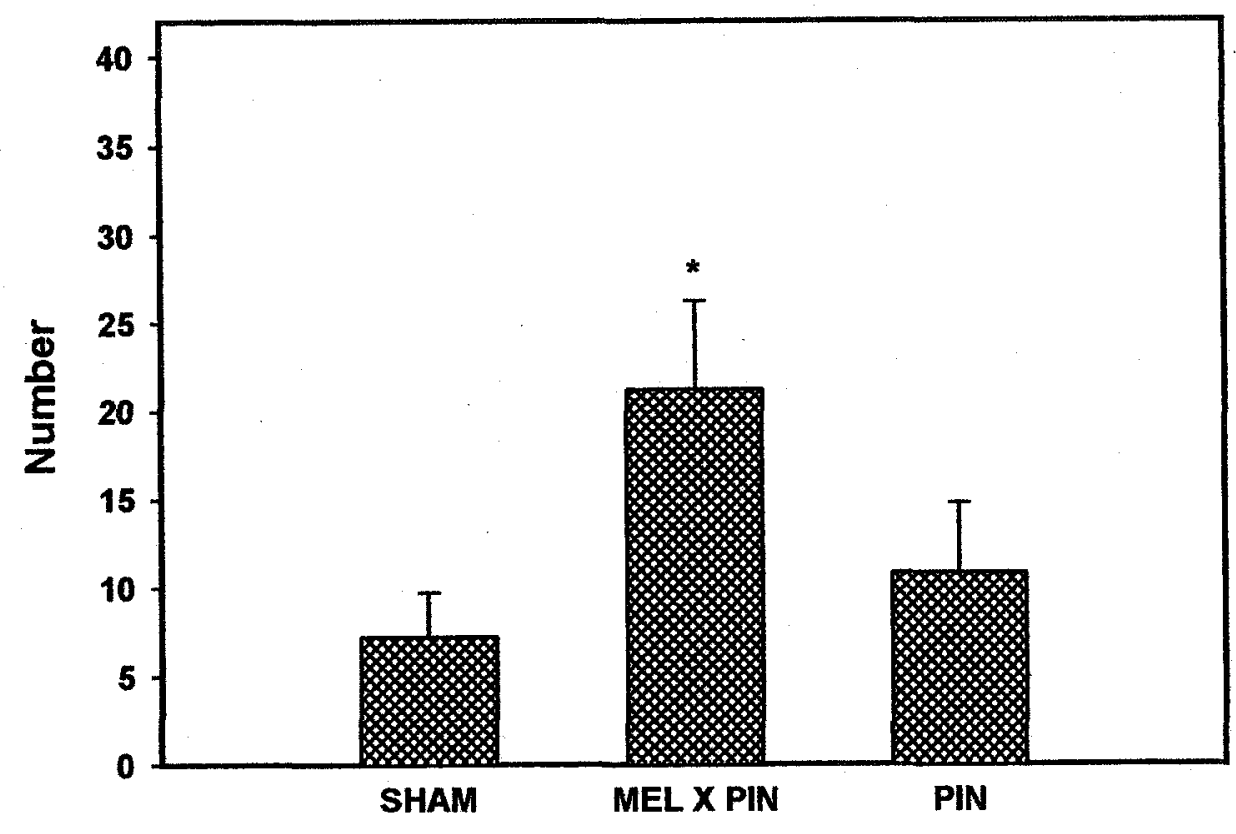

Closed Arm Entries

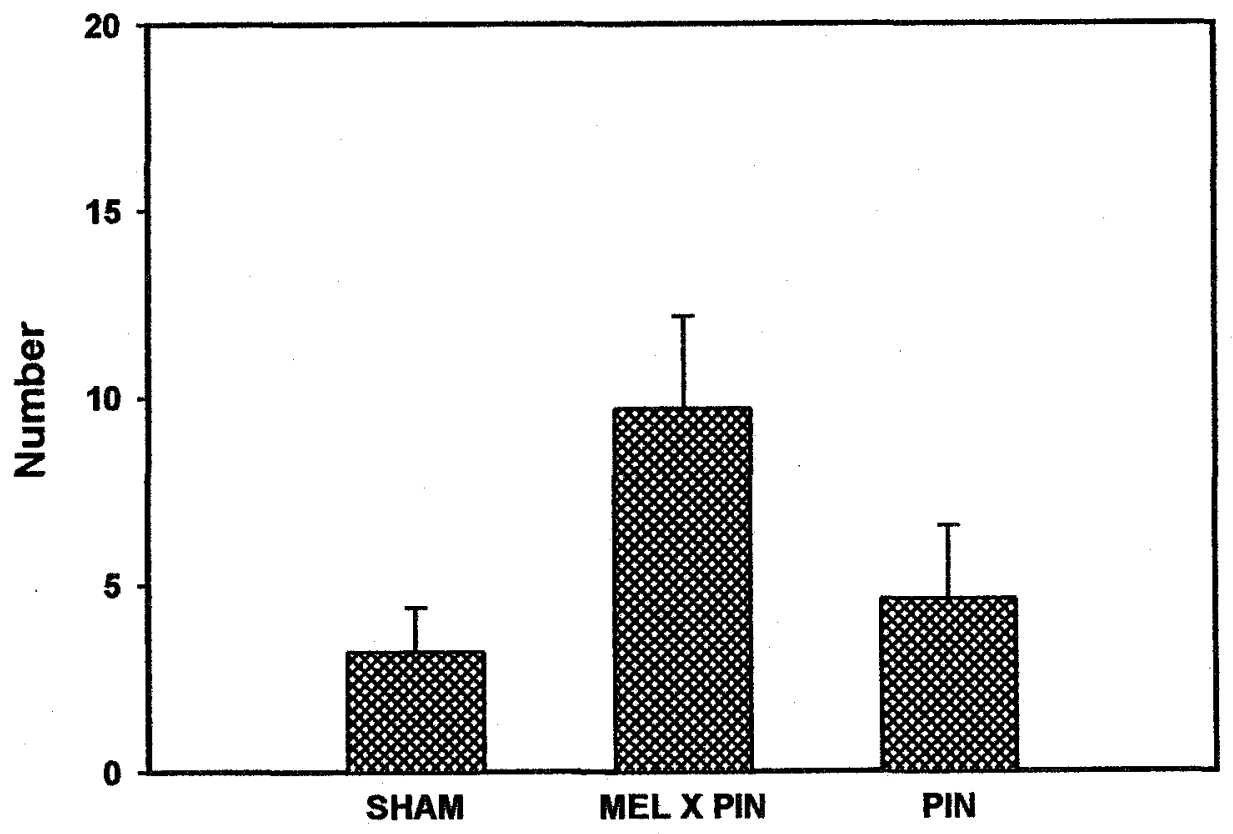


Figure 20. Top Panel: Head dips. At 15 months of age, the groups did not differ in the number of head dips made in the open arms. Error bars represent S.E.M. Bottom Panel: Vertical stretches. The groups did not differ in the number of vertical stretches made in the closed arms. Error bars represent S.E.M. 


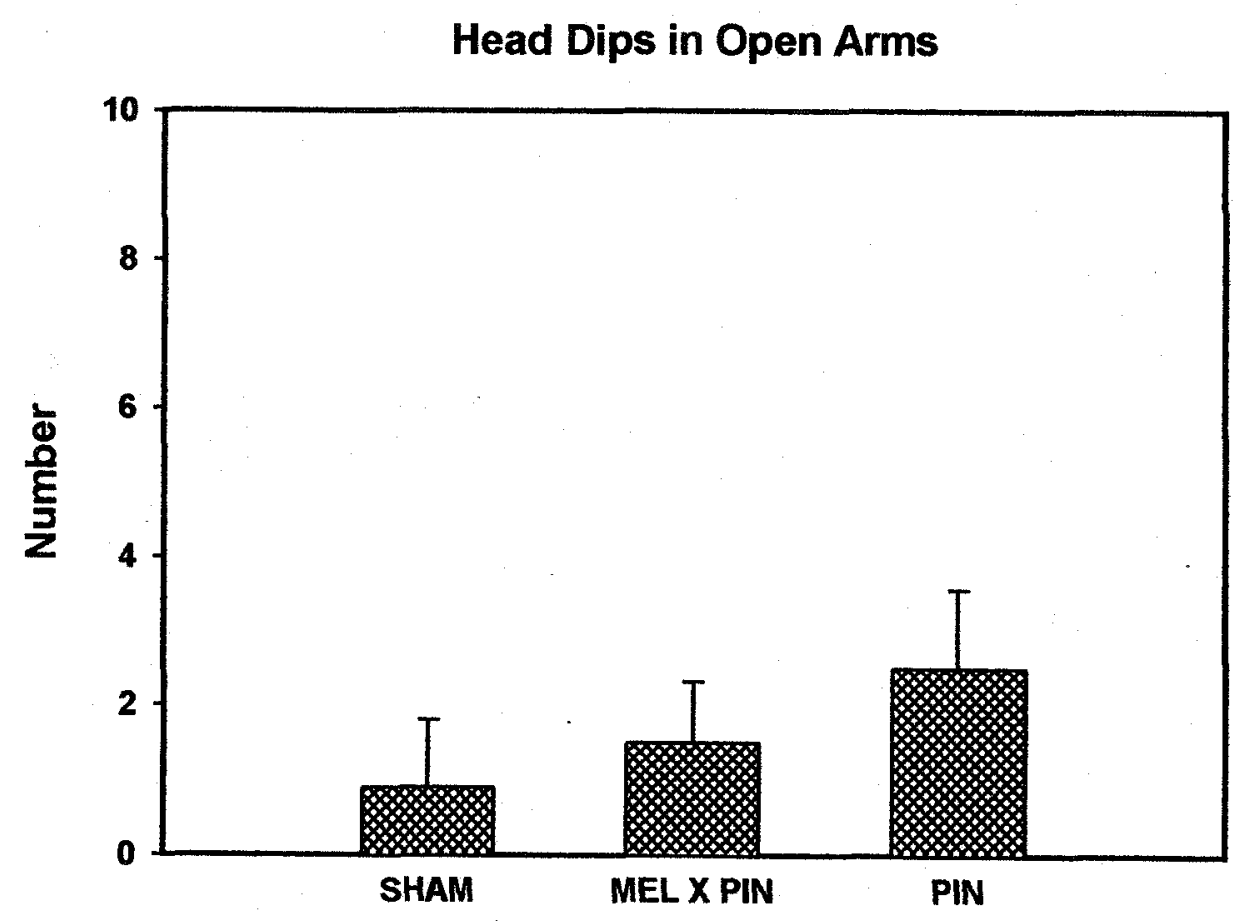

Vertical Stretches in Closed Arms

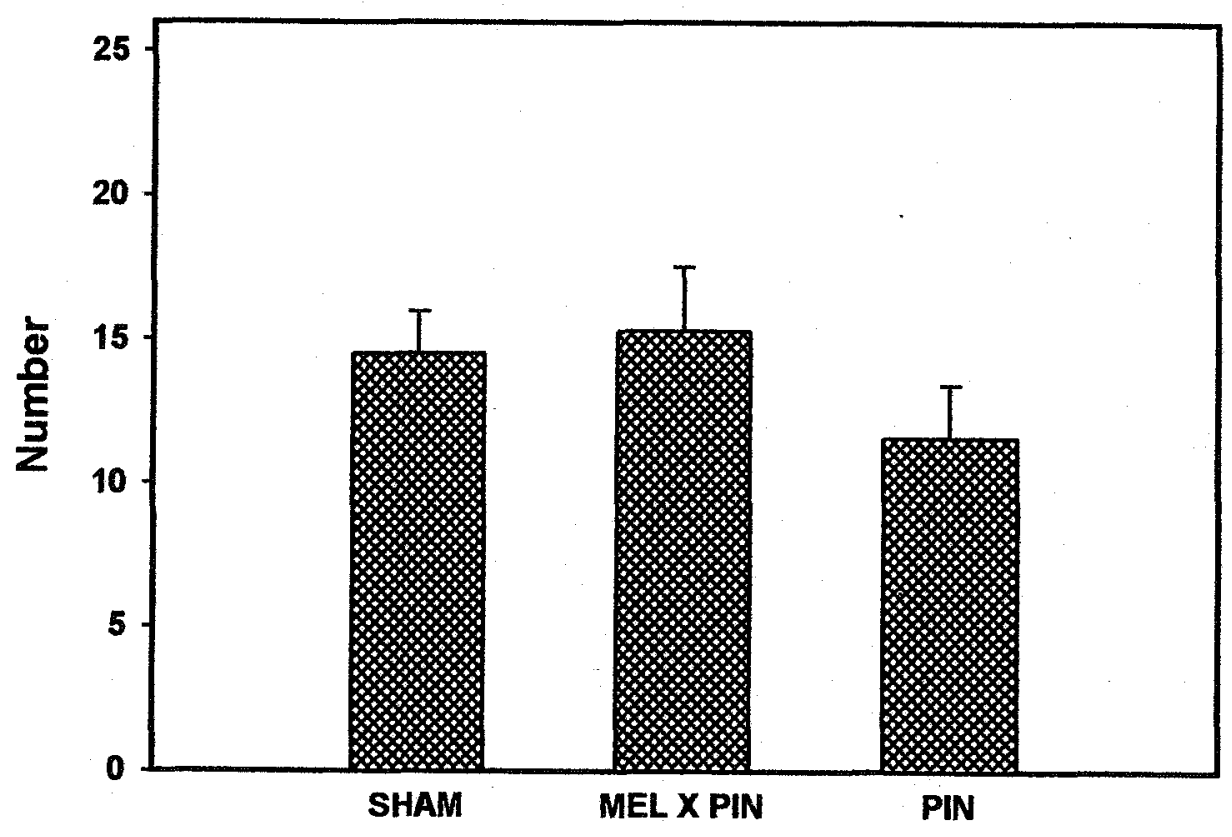


They did not differ on the number of head dips made in the open arms or on the number of vertical stretches made in the closed arms.

Data from all time-points were also compiled together in order to determine whether there were overall group effects and to determine whether there were any age effects on the elevated plus maze task. A significant age effect was found for all of the behaviors that were measured on this task. In terms of the anxiety measures, a significant effect of age was found in the number of open arms that were visited $(F(3,108)=34.43$, $\mathrm{p}<.001$ ) (Figure 21). Tukey's test indicated the 2-month old rats made significantly more entries into the open arms compared to the 7, 11, and 15-month animals $(p<.001)$. Additionally, 7-month old animals also made more entries into the open arms compared to the 15 -month old animals $(p<.001)$.

In addition to the number of entries made into open arms, a significant effect of age was also found for the percentage of total arm entries that were made into the open arms $(F(3,108)=7.37, p<.001)$ (Figure 21$)$. The percentage of overall arm entries made into the open arms was significantly higher in the 2-month compared to the 15 -month old animals $(p<.001)$. There was no significant difference between the 2 -month animals and either the 7-month or 11-month animals in the percentage of open arms visited although this narrowly missed significance $(p=.06$ and $p=.07$ respectively). Additionally, there was no significant difference between the 7,11 , and 15 -month old rats on this measure.

The percentage of time spent on the open arms was also found to be significantly different between the age groups $(F(3,108)=23.63, \mathrm{p}<.001)$ (Figure 22 ). Tukey's test indicated 2-month old rats spent significantly more time on the open arms compared to the 7, 11, and 15- month old rats $(\mathrm{P}<.001)$. Additionally, 7-month old animals spent a 
Figure 21. Top Panel: Number of open entries. Older rats made significantly fewer entries into the open arms compared to the younger rats $(F(3,108)=34.43, p<.001)$. * represents differences compared to the 2-month group. Error bars represent S.E.M. Bottom Panel: Ratio of open entries. The percentage of entries into the open arms was lower in the aged animals compared to the younger animals $(F(3,108)=7.37, p<.001)$. denotes differences between the 15-month and 2-month animals. Error bars represent S.E.M. 

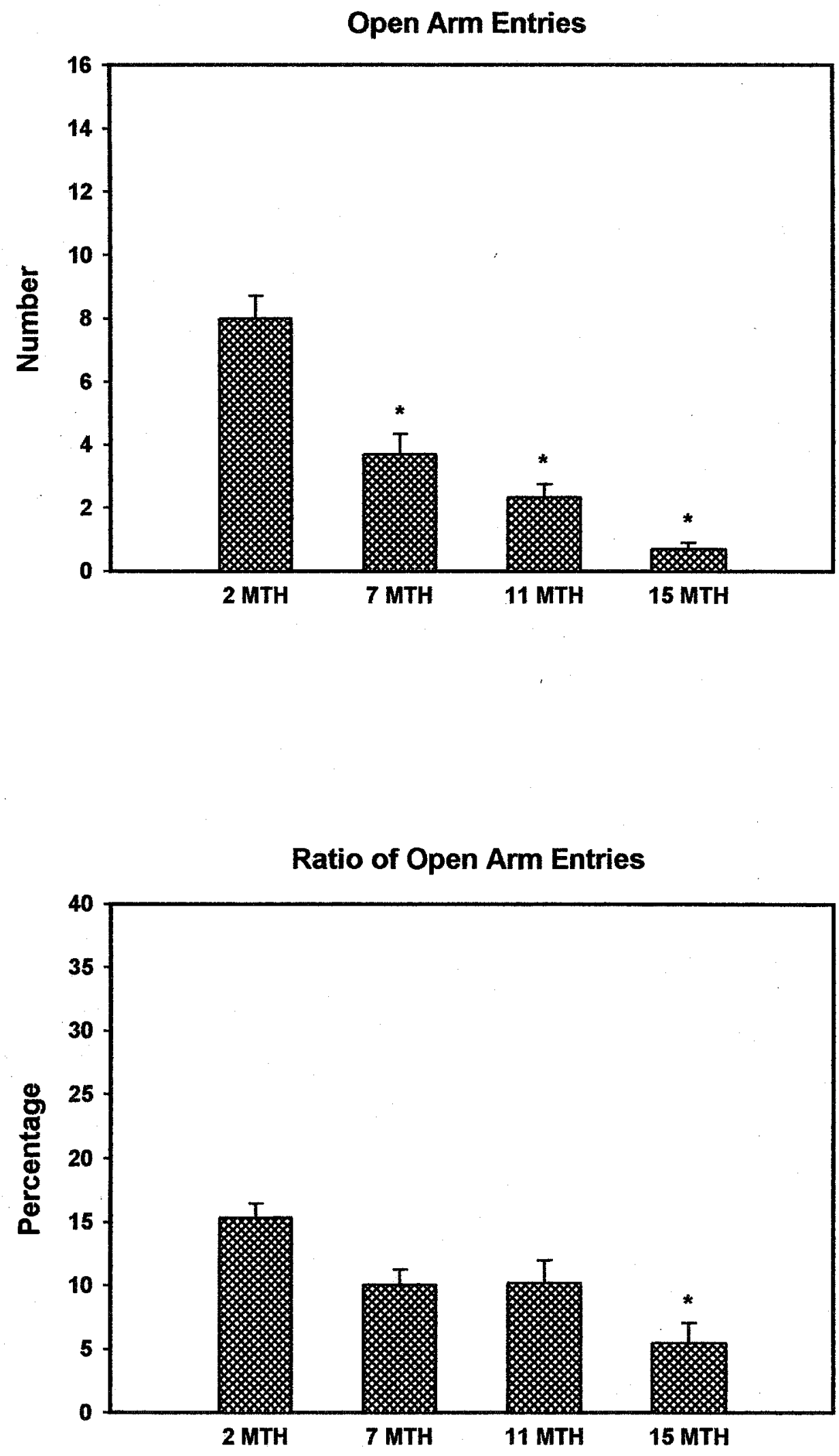
Figure 22. Top Panel: Ratio of time spent on open arms. Young rats spent significantly more time in the open arms compared to the older rats $(F(3,108)=23.63, \mathrm{p}<.001)$. denotes differences compared to the 2-month animals. Error bars represent S.E.M.

Bottom Panel: Ratio of protected stretched attend postures. The older rats made a greater percentage of stretched attend postures from the protected areas of the maze compared to the younger rats $(F(3,108)=19.15, \mathrm{p}<.001)$ * denotes differences compared to the 2 month group. Error bars represent S.E.M. 
Ratio of Time Spent in Open Arms

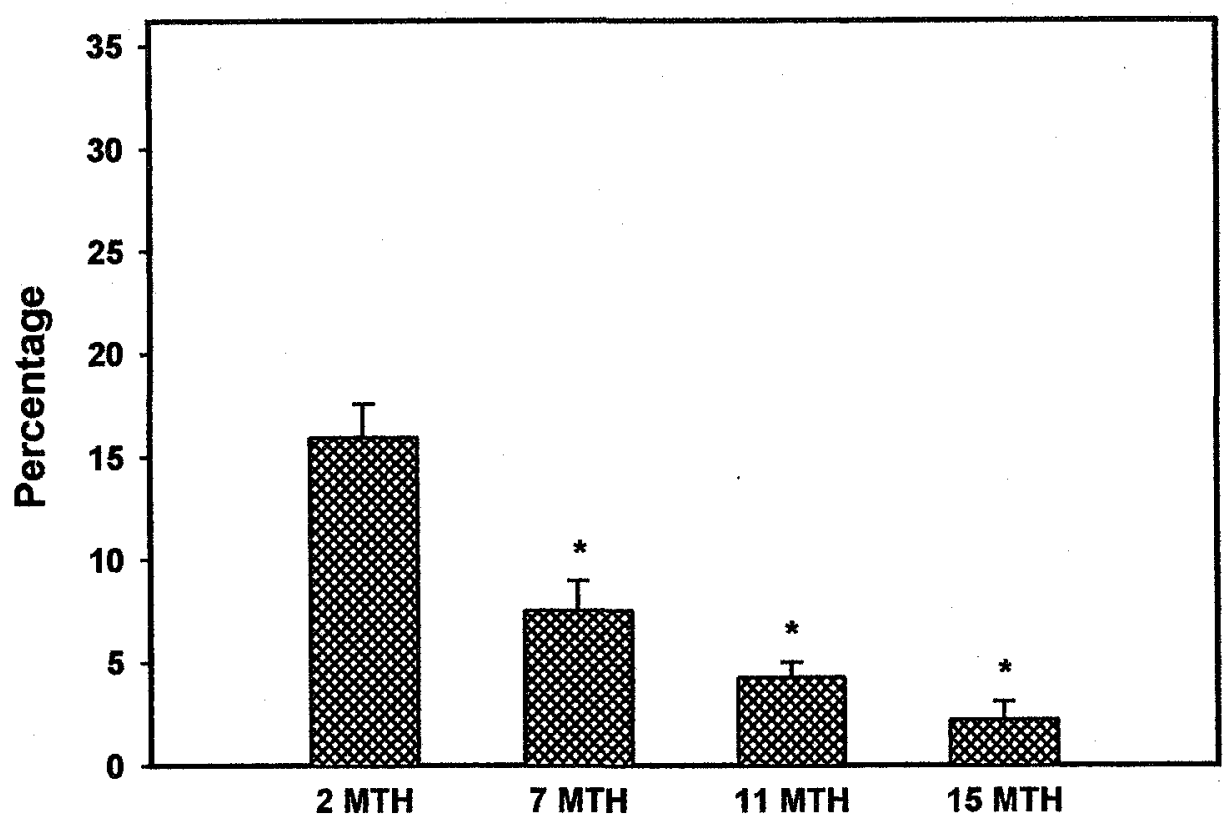

Ratio of Protected Stretched Attend Postures

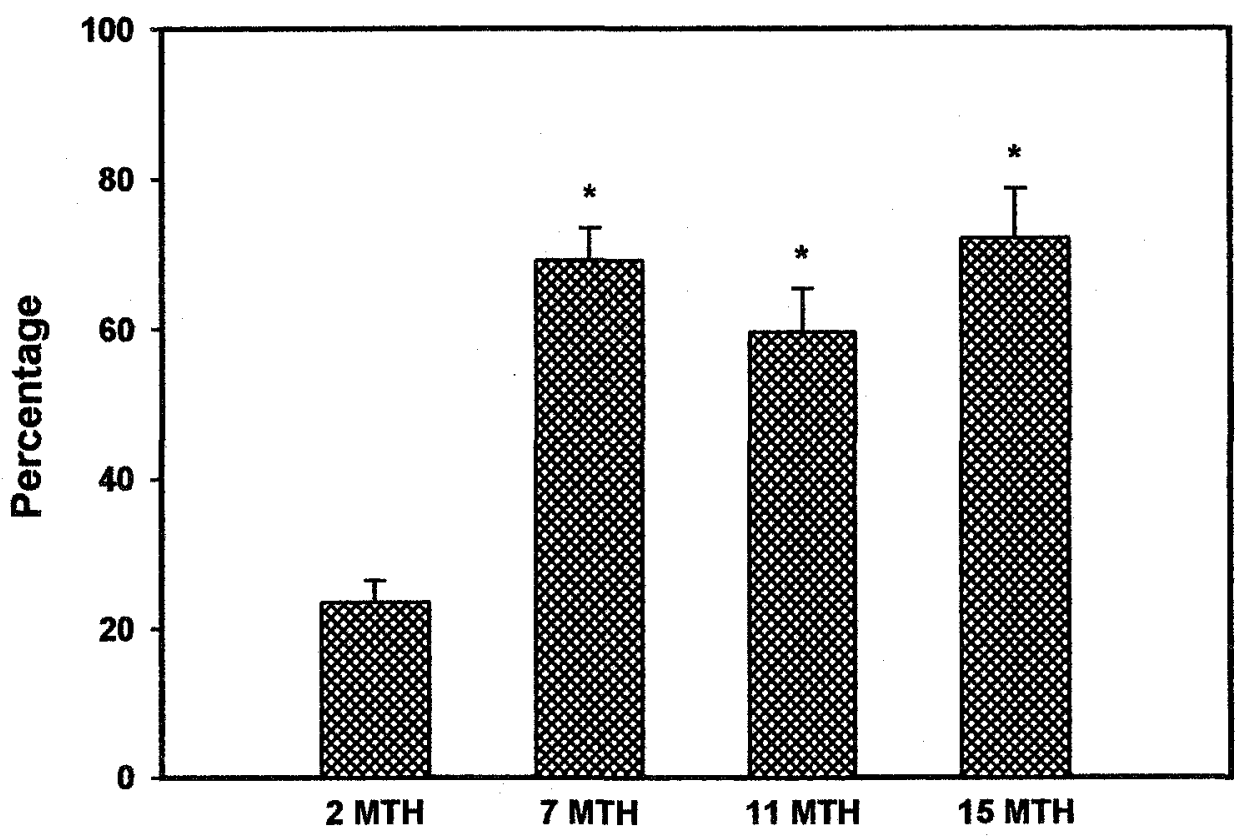


significantly greater proportion of time in the open arms compared to 15 -month old animals $(p<.02)$. No significant difference was found between 7 and 11 -month old rats on the proportion of time spent visiting the open arms. Moreover, 11-month old and 15month old rats did not differ on the proportion of time they spent in the open arms.

In regards to the final anxiety measure, percentage of stretch attend postures from a protected (closed) arm, a significant age effect was also found $(F(3,108)=19.15$, $\mathrm{p}<.001$ ) (Figure 22). Tukey's test revealed, two month old rats performed significantly fewer stretch attend postures from the closed arms than the older age groups $(p<001)$. No significant difference was found between the 7,11 and 15-month old animals. A significant difference was found between the age groups on the percentage of time spent in the central hub $(F(3,108)=40.93, \mathrm{p}<.001)$ (Figure 23). Tukey's test indicated that the 2-month old rats spent significantly more time in the central hub than the older animals $(p<.001)$. Additionally, the post-hoc comparison revealed that the rats in the 15-month group spent significantly less time on the center hub compared to the younger age groups $(p<.001)$. No significant difference was found between the 7 and 11-month animals on the proportion of time spent in the center hub.

Locomotor activity was also found to differ between the age groups. Specifically, the age groups differed in the total number of arms they entered $(F(3,108)=27.80$, $\mathrm{p}<.001)$ (Figure 24). A tukey's test revealed that the 2-month rats entered significantly more arms during their testing session than the rats in the older age groups $(\mathrm{p}<.001)$. Compared to the other three groups, the 15-month group visited significantly fewer arms during the testing session $(p<.001)$. No difference was found between the 7 and 11month old animals on the total arm entries. The number of entries made into the closed 
Figure 23. Ratio of time spent in central hub. The 2-month old rats spent significantly more time in the central hub compared to the older age groups $(F(3,108)=40.93, p<.001)$. * denotes differences compared to the 2-month group. Error bars represent S.E.M. 
Ratio of Time in Center Hub

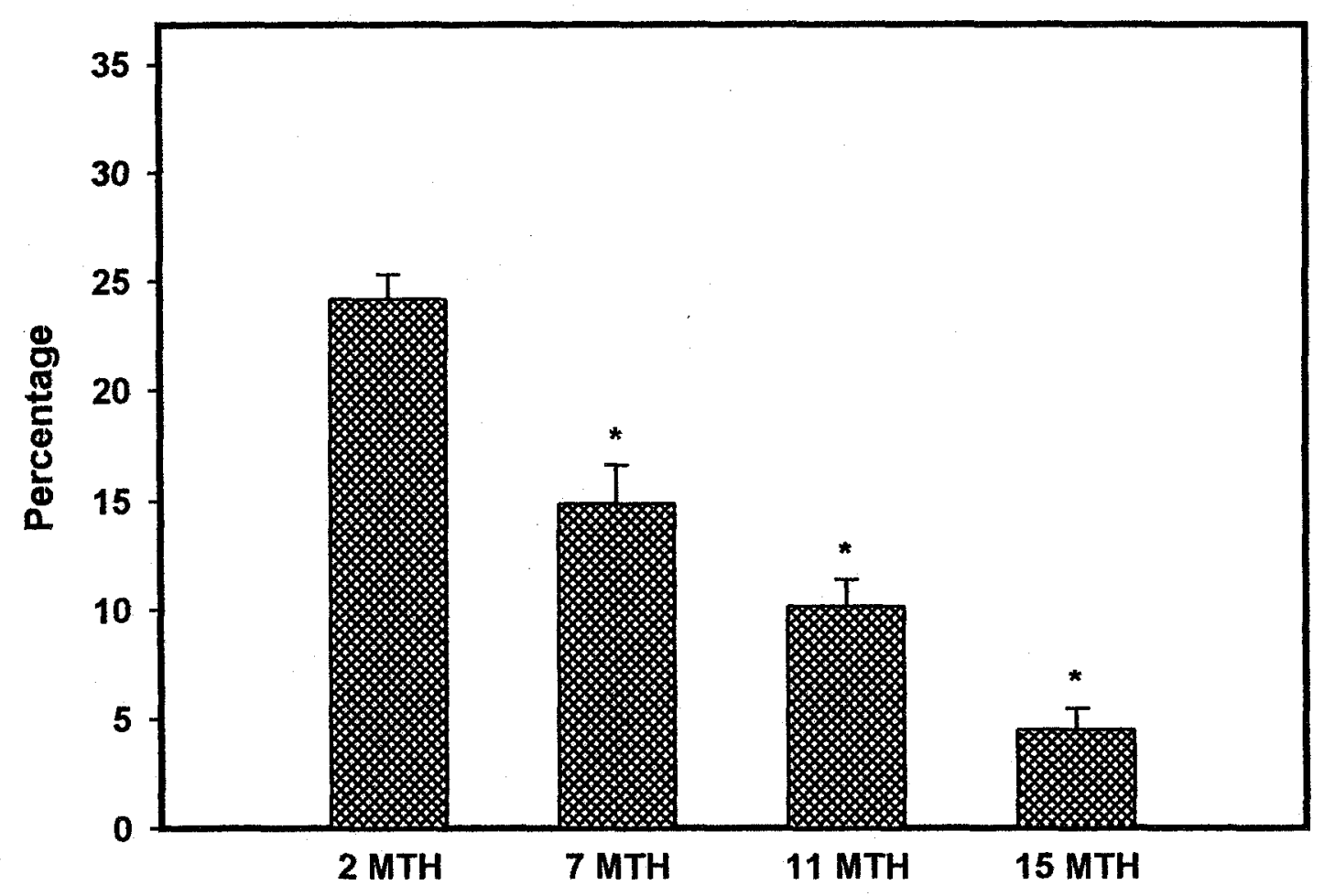


arms also differed between the groups $(\mathrm{F}(3,108)=16.82, \mathrm{p}<.001)$ (Figure 24). A post-hoc comparison indicated that the rats in the 2-month group visited a greater number of closed arms compared to the 11 and 15-month groups $(p<.001)$. In addition, the 15-month animals made significantly fewer closed arm entries than the other groups $(p<.001)$. No difference was found between the 2 and 7-month old animals. Moreover, 7 and 11-month old animals did not differ in the number of closed arms that were visited during the testing session.

Exploration on the elevated plus maze also differed among the age groups. The number of head dips (measure of exploration in the open arms) significantly differed between the age groups $(F(3,108)=32.05, \mathrm{p}<.001)$ (Figure 25). The younger animals exhibited a greater number of head dips than the older age groups $(p<.001)$. The 15month animals made significantly fewer head dips than either the 2 or 7 -month old animals. No significant difference was found between the 7 and 11-month old rats. As well, rats in the 11-month group did not differ from the 15-month rats. Similar to the number of head dips on the open arms, the number of vertical stretches in the closed arms also differed among the age groups $(F(3,108)=16.04, p<.001)$. As shown in Figure 25, rats in the 2-month group exhibited greater exploration in the closed arms than the 11 and 15-month old age groups $(\mathrm{p}<.001)$. The 7 -month old animals did not differ from the 2month animals in the number of vertical stretches that were made in the closed arms. Overall, the 15-month rats made significantly fewer vertical stretches compared to the other age groups $(\mathrm{p}<.001)$.

In summary, a significant main effect of age was found for all behavioral measures. Specifically, as the rats aged, they showed an increase in anxiety related 
Figure 24. Top Panel: Total entries. Older age groups made significantly fewer arm entries compared to the younger animals $(F(3,108)=27.80, p<.001) .{ }^{*}$ denotes differences compared to the 2-month group. Error bars represent S.E.M. Bottom Panel: Number of closed entries. Older rats entered a fewer number of closed arms compared to the young rats $(F(3,108)=16.82, p<.001)$. ${ }^{*}$ denotes differences compared to the 2 -month group. Error bars represent S.E.M. 
Total Entries

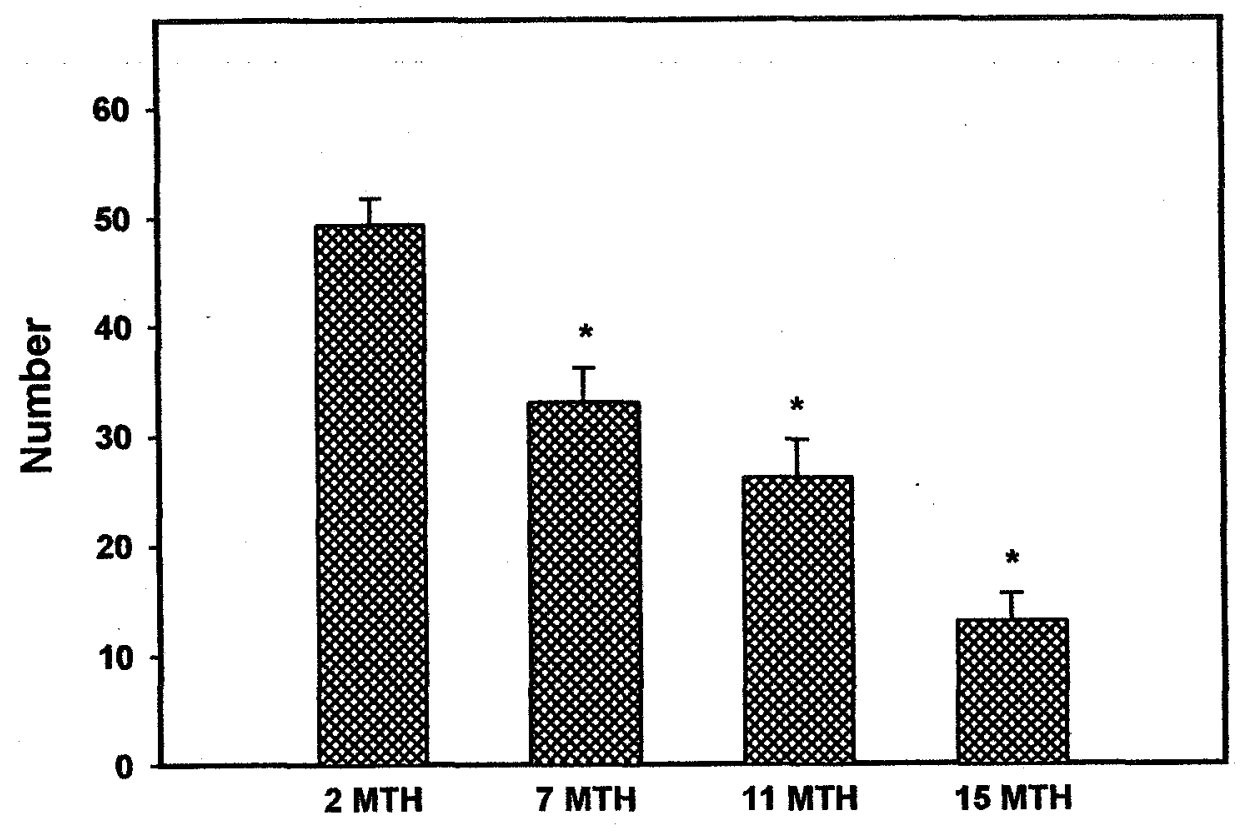

Closed Arm Entries

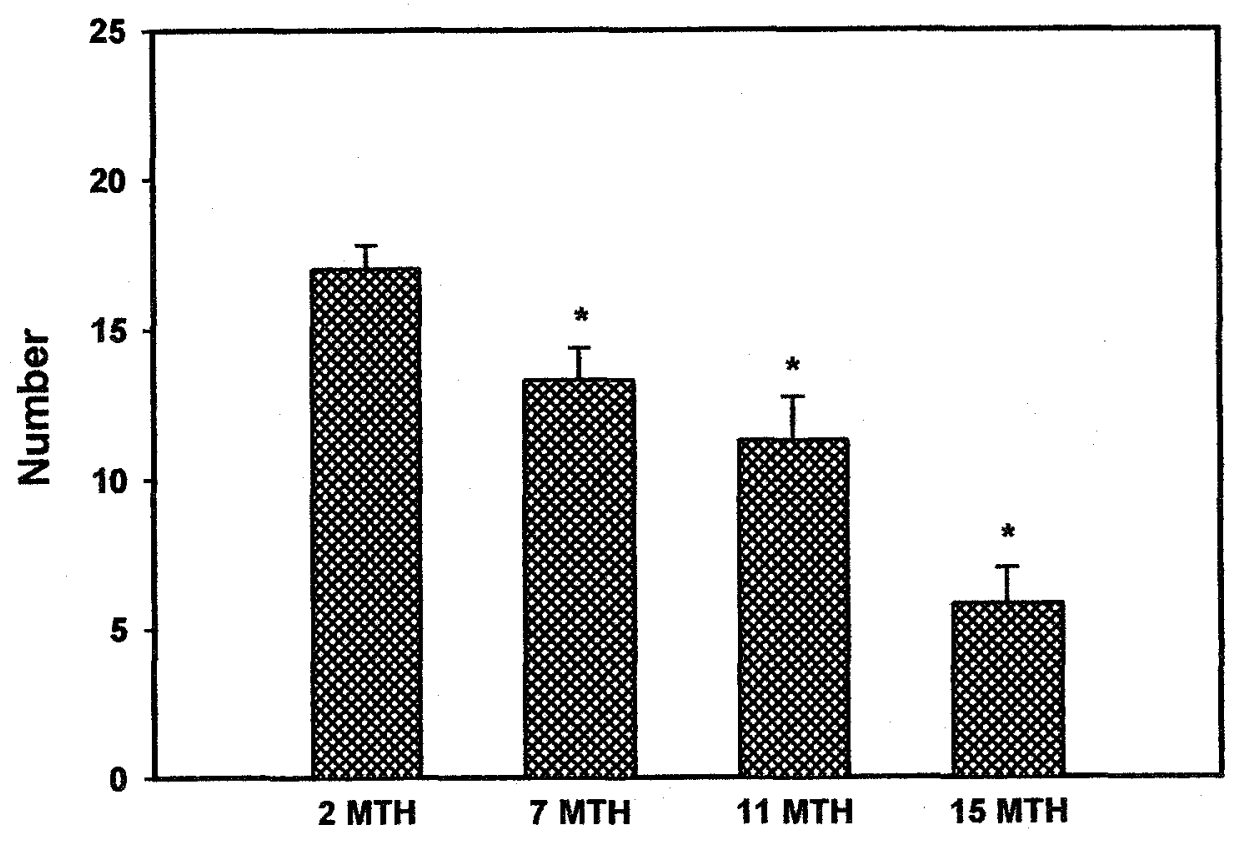


Figure 25. Top Panel: Head dips. Younger animals made a greater number of head dips in the open arms compared to the older rats $(F(3,108)=32.05, \mathrm{p}<.001)$. * denotes differences between the 15-month group and the 2 and 7-month age groups. Error bars represent S.E.M. Bottom Panel: Vertical stretches. Younger animals made a greater number of vertical stretches in the closed arms compared to the older rats $(F(3$, $108)=16.04, \mathrm{p}<.001) *$ denotes differences compared to the 2-month group. Error bars represent S.E.M. 

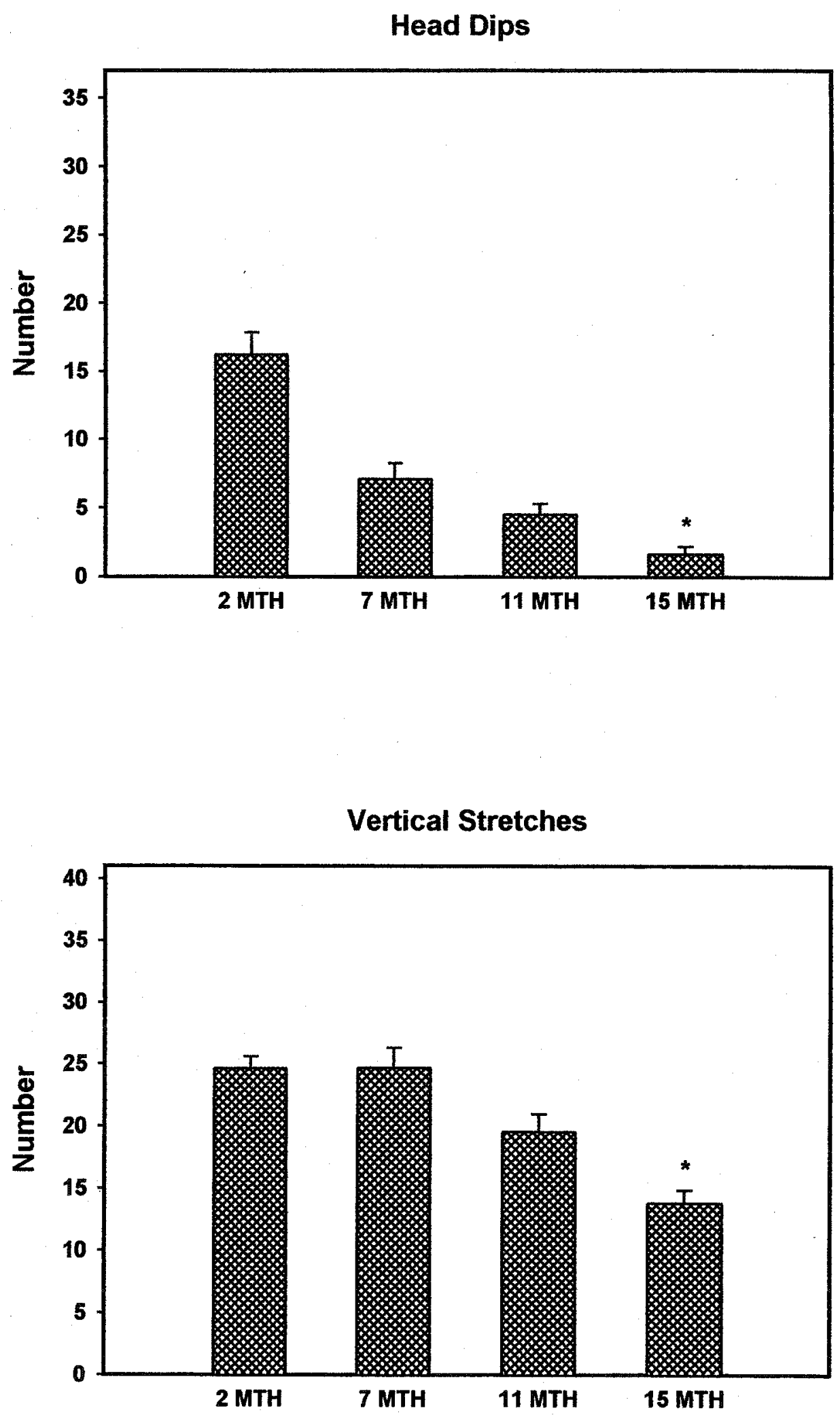
behaviors. As well, older rats made significantly fewer entries into both the closed and open arms. Hence, their locomotor activity was decreased. Exploration was also decreased in the older animals. No significant main effect of group was found nor a significant age $\mathrm{X}$ group effect for any of the measures.

\section{Morris Water Maze}

Following exposure to the elevated plus maze, animals from three different timepoints $(2,7$, and 11 months) were tested on the working memory version of the Morris Water Maze. Animals received 5 daily trials for six days. Both latency and distance to find the hidden platform were recorded for each swim and the latency and distance from the last four trials were averaged for each daily session. These averages were then used for statistical analysis. For all time-points, a mixed factorial ANOVA was performed with group (sham, melatonin supplemented, and pinealectomized) as a between- subjects factor and test day (1-6) as a within-subjects factor. Post-hoc comparisons were performed using Tukey's test.

At the 2-month time-point, no significant differences were found between the groups for both latency and distance to reach the platform (Figures 26 and 27). A significant effect of day was found for both latency $(F(5,135)=19.99, p<.0001)$ and distance $(F(5,135)=23.72, p<.0001)$ to find the platform. Specifically, all rats exhibited shorter latencies and path lengths over test days.

Similar to the 2-month age group, no significant differences were found between the groups on either latency or distance to find the hidden platform at the 7-month time- 
Figure 26. Hidden platform water maze. At 2-months post-surgery, latency to find the hidden platform did not differ between the groups. All groups demonstrated shorter latencies across test days $(\mathrm{F}(5,135)=19.99, \mathrm{p}<.0001)$. Error bars represent S.E.M. 


\section{Latency to Find Platform}

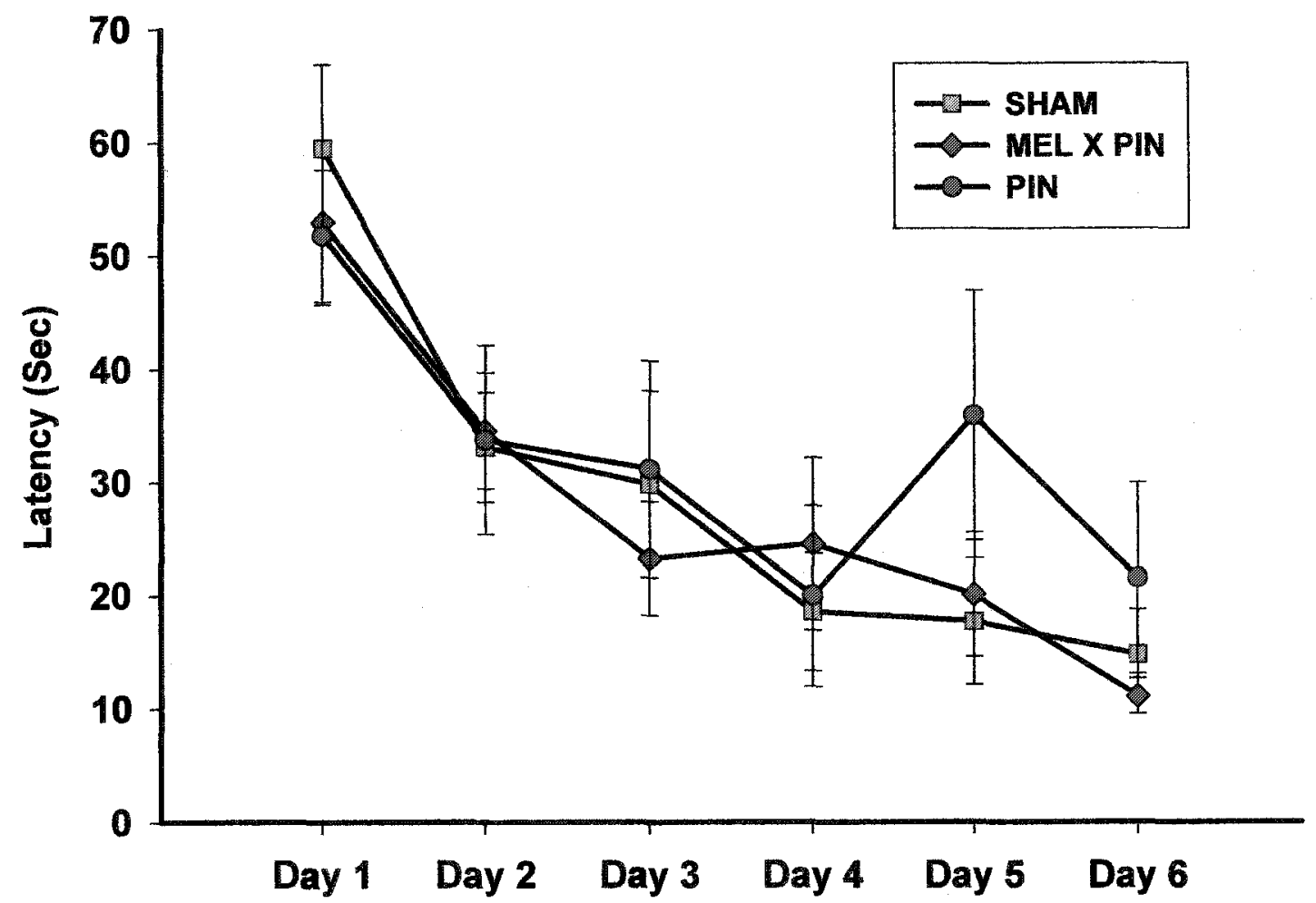


Figure 27. Hidden platform water maze. At 2-months post-surgery, the groups did not differ in path-length to reach the platform. All rats exhibited shorter path-lengths over test days $(F(3,135)=23.72, p<.0001)$. Error bars represent S.E.M. 


\section{Pathlength to Find Platform}

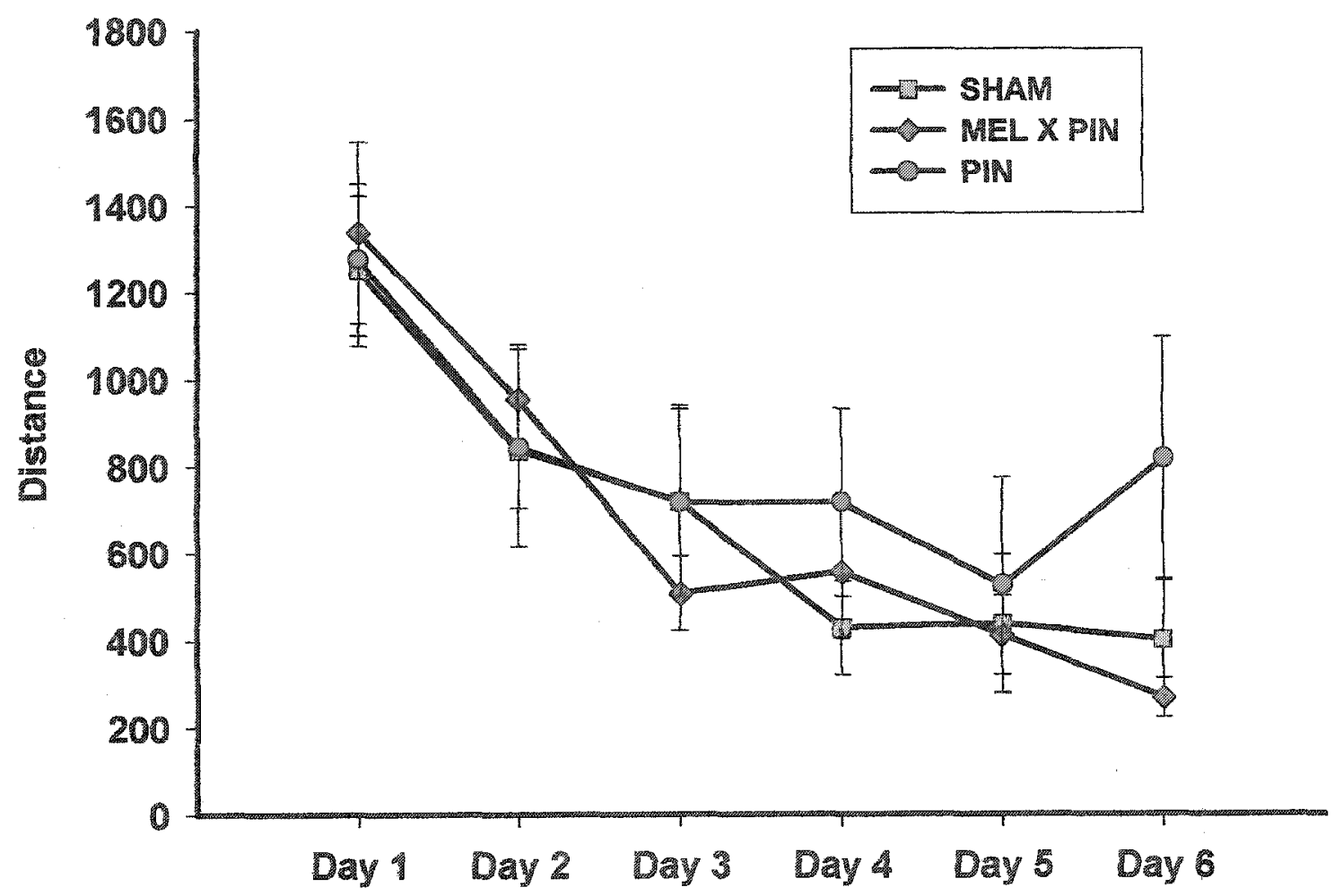


point (Figures 28 and 29). All rats exhibited shorter latencies $(F(3,135)=48.33, p<.0001)$ and path lengths $(\mathrm{F}(3,135)=45.43, \mathrm{p}<.0001)$ over test days.

As illustrated in Figures 30 and 31, no significant differences were found between the groups on either latency or distance to reach the platform at the 11-month time-point. Similar to the earlier time-points, a significant effect of day was found for both latency $(F(3,135)=58.09, \mathrm{p}<.0001)$ and path-length $(\mathrm{F}(3,135)=66.19, \mathrm{P}<.0001)$ to find the platform.

A compilation of all the data from the 2,7 , and 11 time-points revealed a significant overall effect of age $(F(2,81)=3.21, \mathrm{p}<.05)$. As illustrated in Figure 32, 2month old animals took significantly less time to reach the platform than the 11-month old animals (Tukey HSD, $\mathrm{p}<.05$ ). No significant difference in latency was found between the 7 and 11 month old animals. No significant effect of group was found nor was there a significant Age X Group effect.

Additionally, a significant effect of day was also found $(F(5,405)=113.63$, $p<.0001$ ). As shown in Figure 32, animals from all 3 age groups exhibited improvement over testing days. Moreover, a significant Day X Age interaction was found $(F(10,405)=$ 2.31, $\mathrm{p}<.01$ ). A one-way ANOVA revealed that 2-month old animals had significantly lower latencies on the first $(\mathrm{F}(2,87)=5.20, \mathrm{p}<.007)$ and second $(\mathrm{F}(2,87)=10.08$, $\mathrm{p}<.0001$ ) testing day compared to the 11 -month old animals. Latency did not differ between the age groups on days 3-6. No significant Day X Group interaction was found nor was there a significant Day X Age X Group interaction.

An ANOVA on a compilation of all the path-length data for all age groups, revealed no significant main effect of age or group. Moreover, there was no significant 
Figure 28. Hidden platform water maze. At 7 months of age, platform location latencies did not differ between the groups. All groups exhibited shorter latencies across test days $(F(3,135)=48.33, p<.0001)$. Error bars represent S.E.M. 


\section{Latency to Find Platform}

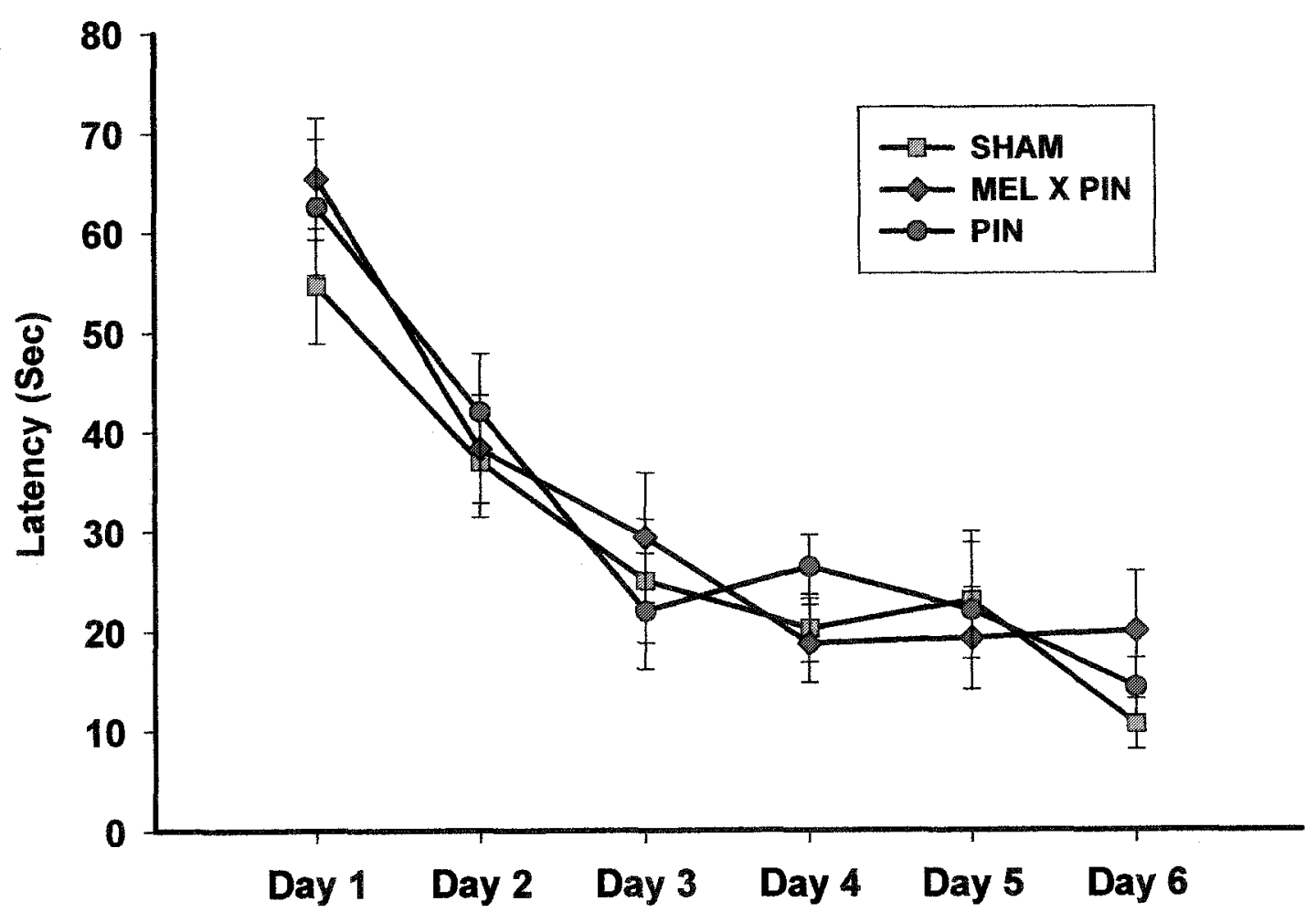


Figure 29. Hidden platform water maze. At 7-months post-surgery, groups did not differ in path-length to reach the platform. All rats exhibited shorter path-lengths across test days $(F(3,135)=45.43, p<.0001)$. Error bars represent S.E.M. 


\section{Pathlength to Find Platform}

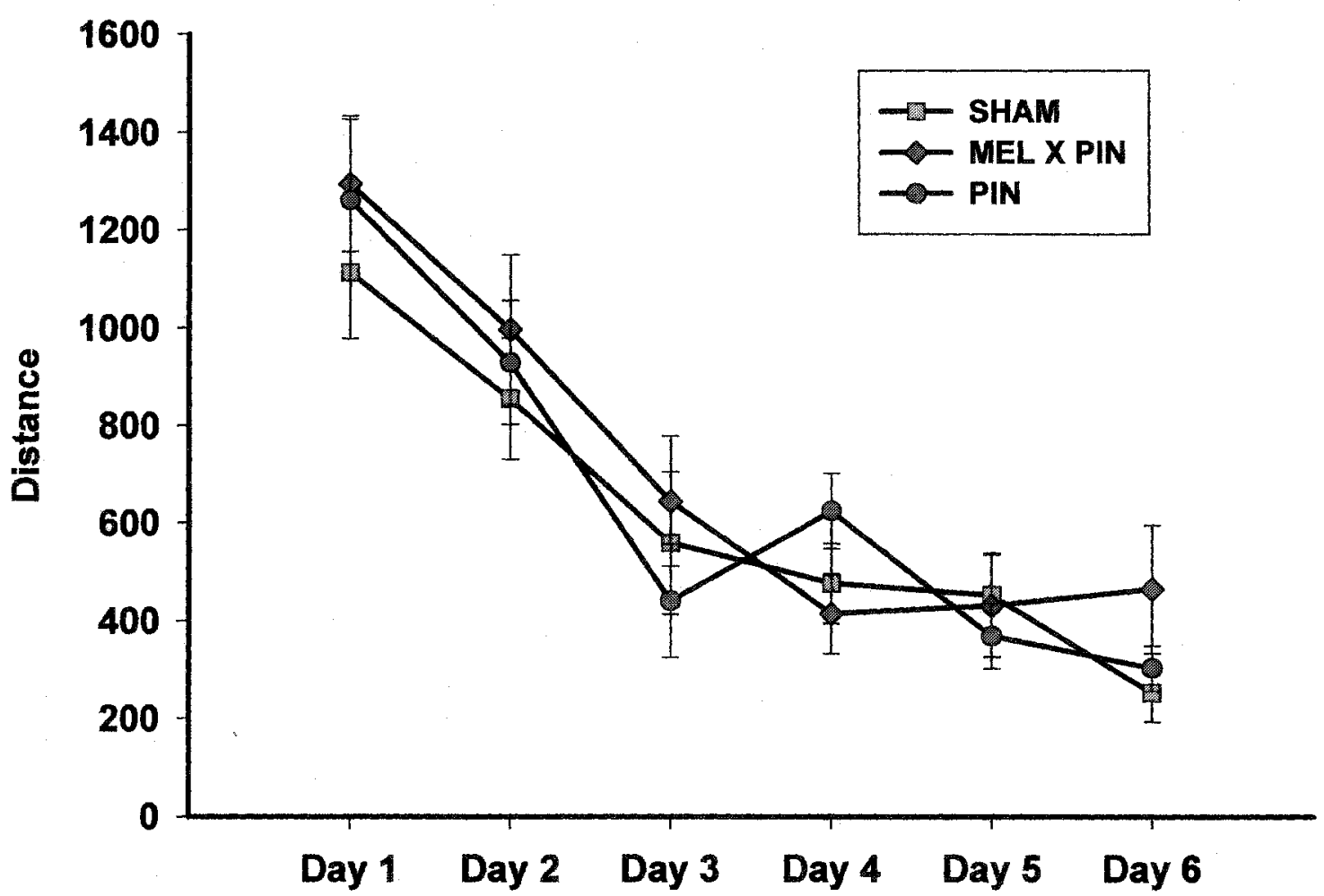


Figure 30. Hidden platform water maze. At 11-months post-surgery, no group differences were observed in latency to reach the platform. All groups exhibited shorter latencies across test days $(\mathrm{F}(3,135)=58.09, \mathrm{p}<.0001)$. Error bars represent S.E.M. 


\section{Latency to Find Platform}

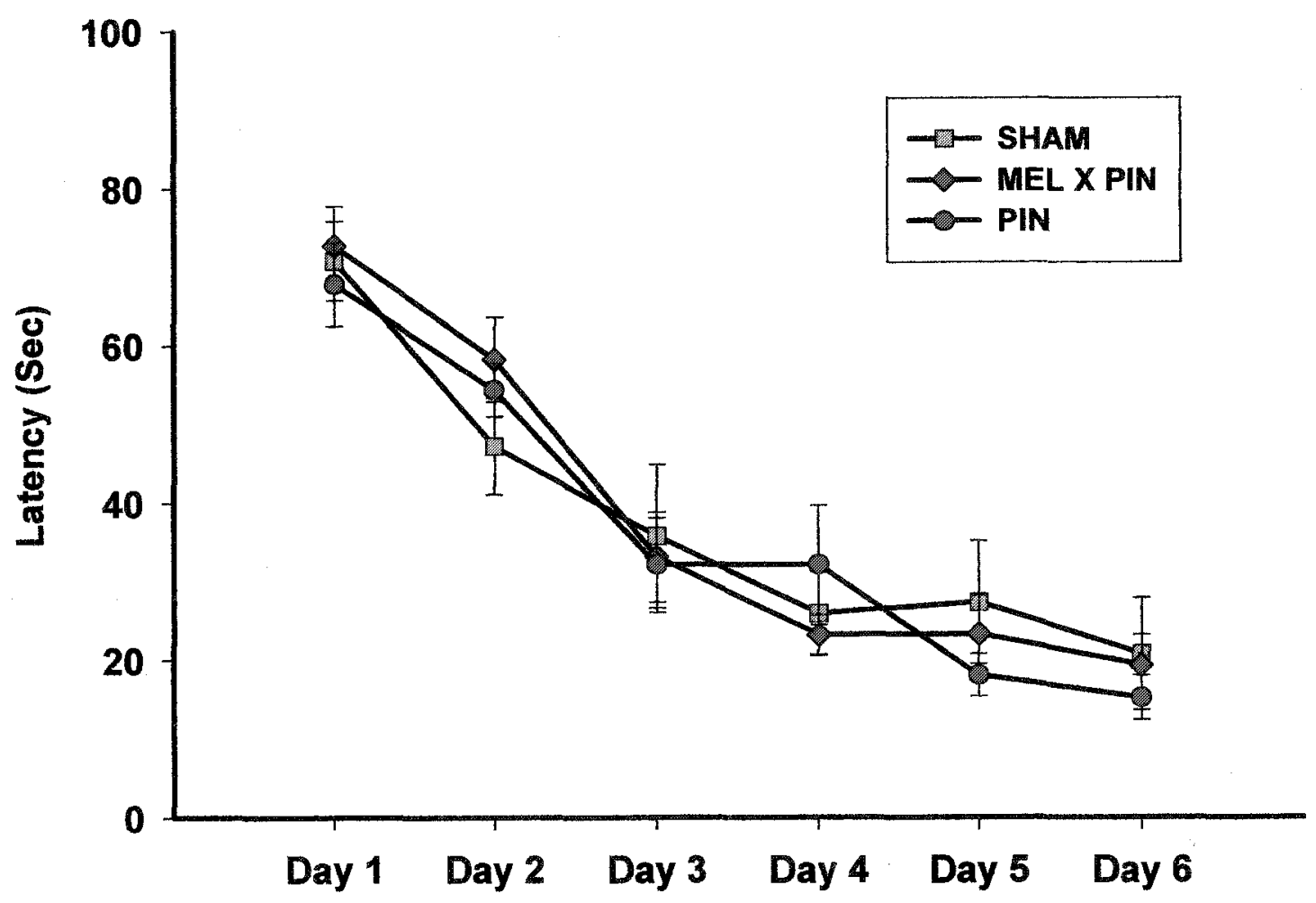


Figure 31. Hidden platform water maze at 11-months post-surgery. No group differences were found in path-length to find the hidden platform. All rats exhibited shorter path-lengths across test days $(F(3,135)=66.19, p<.0001)$. Error bars represent S.E.M. 


\section{Pathlength to Find Platform}

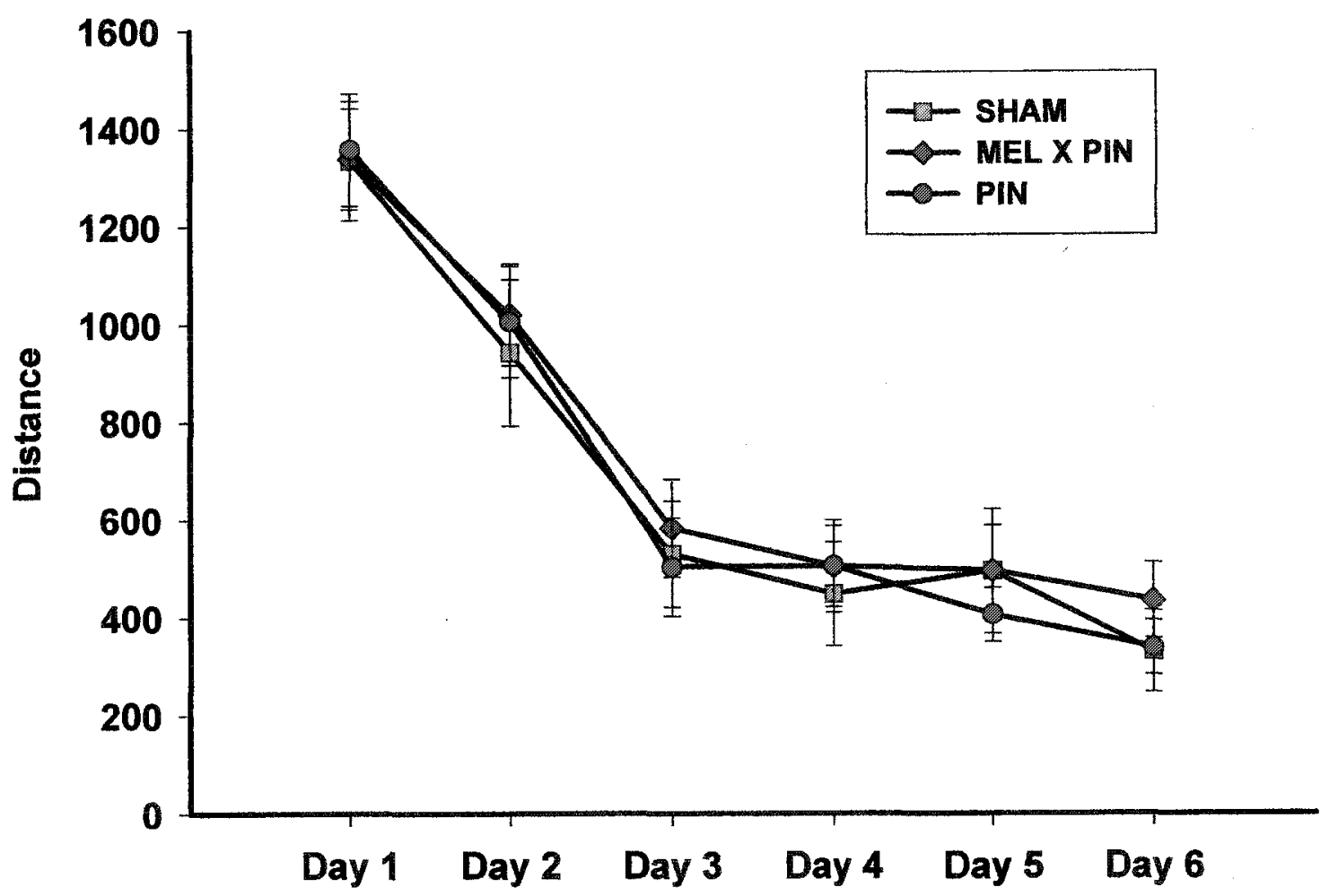


Figure 32. Age effects on platform location latency. Older animals had longer latencies compared to the young rats $(\mathrm{F}(2,81)=3.21, \mathrm{p}<.05)$. * denotes differences between 2 month and 11-month age groups. All rats exhibited improvement across test days (F $(5,405)=113.63, \mathrm{p}<.0001)$ 


\section{Latency to Find Platform}

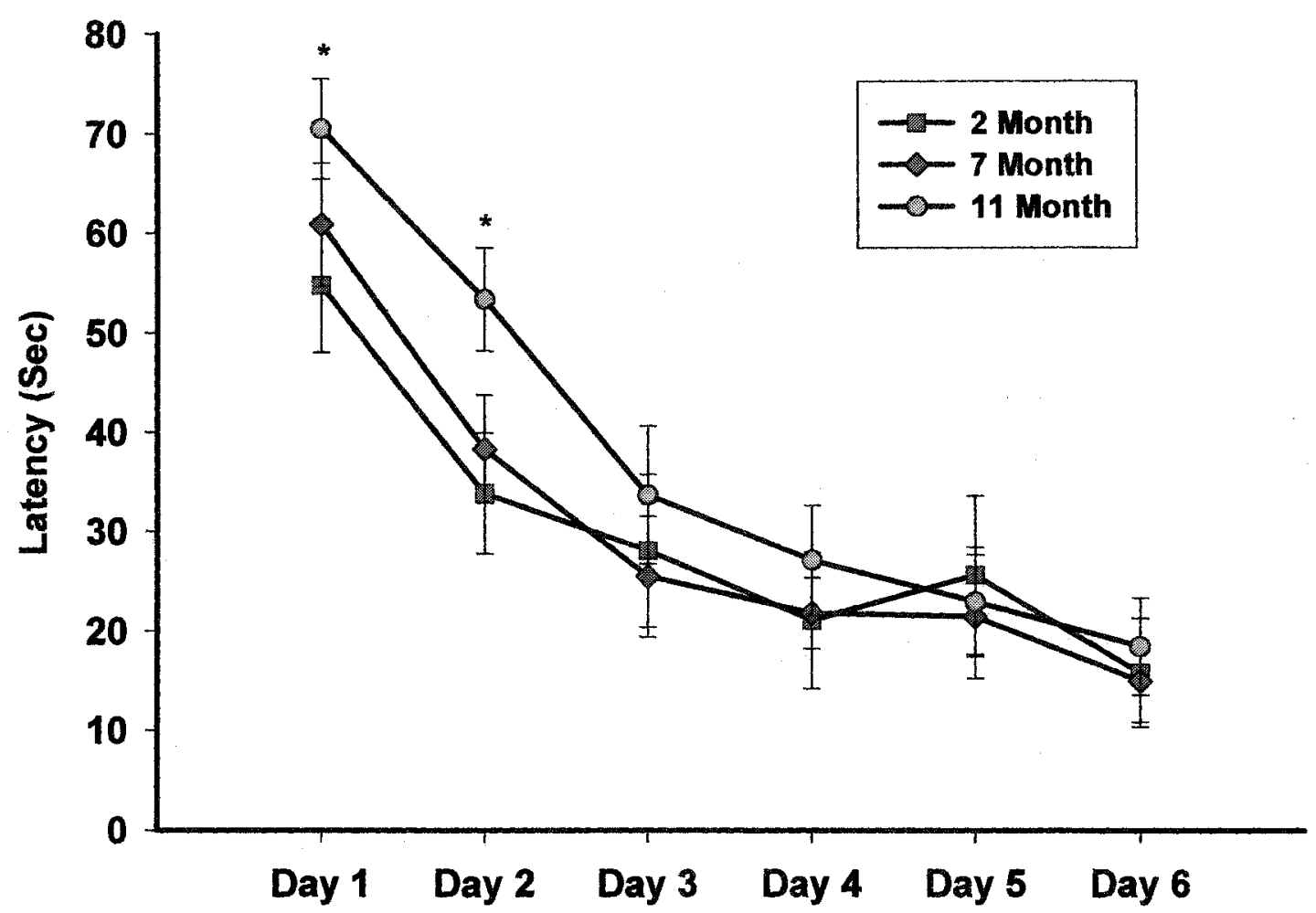


Age X Group effect. The ANOVA did reveal a significant effect of day $(\mathrm{F}(5,405)=$ $119.54, \mathrm{p}<.0001$ ) (Figure 33). Specifically, animals in the 3 age groups exhibited shorter path-lengths across testing sessions. No significant Day X Group interaction was found nor was there a significant Day X Age interaction. Moreover, the ANOVA indicated that there was no significant Day X Group X Age interaction.

\section{Histology}

\section{CA1 Cell Number}

Tissue sections containing the hippocampus were stained with hematoxylin. At each time-point, cells were counted using stereological techniques, which yielded unbiased estimates of hippocampal CA1. Results were analyzed by one-way ANOVA with group (sham, melatonin supplemented, and pinealectomized) as a between subjects factor. Post-hoc comparisons were performed using Tukey's test.

The results for CA1 cell number are shown in Figure 34. Figure 35 is a photomicrograph illustrating CAl of a sham, melatonin supplemented, and pinealectomized rat. Two months post-surgery, an ANOVA revealed that CA1 cell number did not differ between the groups. At 4-months post-surgery, an ANOVA revealed significant differences between the groups $(F(2,15)=15.50, p<.0001)$. The pinealectomized rats had significantly fewer CA1 cells than the sham animals $(74 \%$ of sham) (Tukey HSD, $\mathrm{p}<.0001$ ). In addition, Tukey's test indicated that the melatonin supplemented rats had significantly fewer CA1 cells than the sham animals $(83 \%$ of sham $)(p<.006)$. No significant difference in cell number was found between the melatonin supplemented and pinealectomized animals. 
Figure 33. Age effects on platform location path-length. No significant age effect was found for path-length to reach the hidden platform. All age groups exhibited shorter pathlengths across test days $(F(5,405)=119.54, p<.0001)$. Error bars represent S.E.M. 


\section{Pathlength to Find Platform}

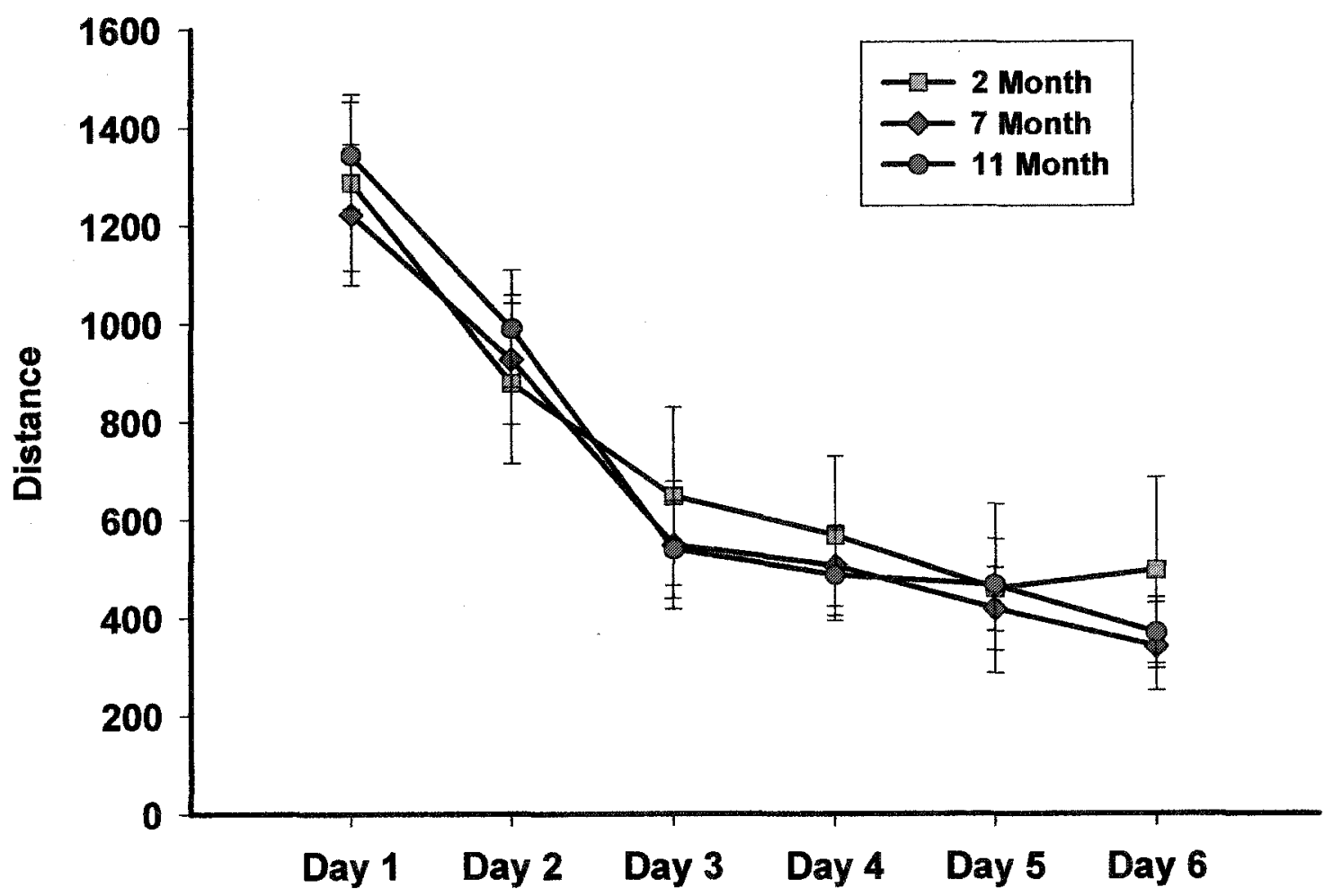


Figure 34. Estimated hippocampal CA1 cell counts. Pinealectomy caused CA1 cell loss 4 months post-surgery. This was prevented by melatonin supplementation. Error bars represent S.E.M. 


\section{CA1}

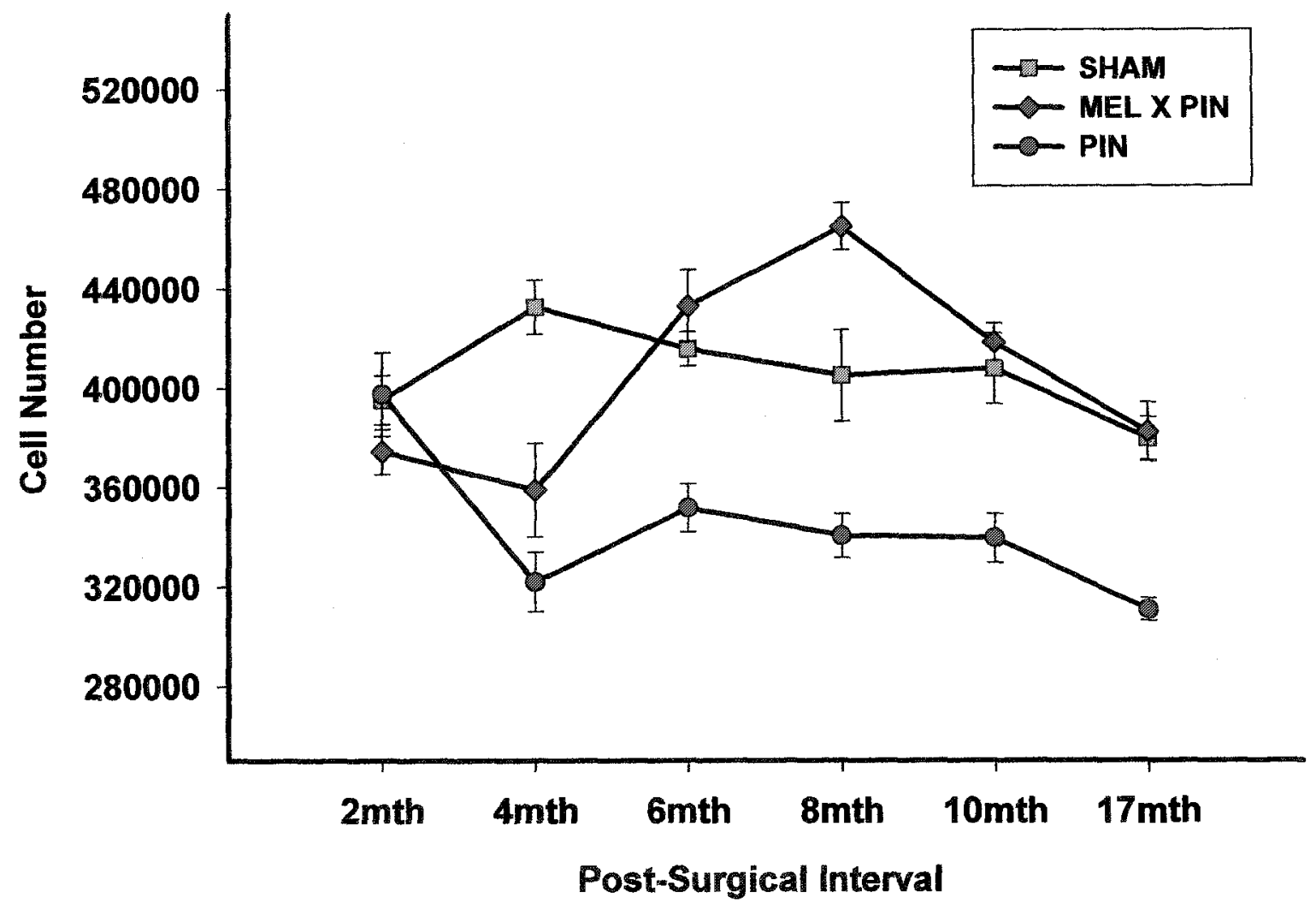


Figure 35. Top Panel: Photomicrograph of hippocampal CA1 pyramidal cell layer at 20X magnification from a sham rat. Middle Panel: Photomicrograph of hippocampal CA1 pyramidal cell layer from a melatonin supplemented rat. Bottom Panel: Photomicrograph of hippocampal CA1 pyramidal cell layer from a pinealectomized rat. 

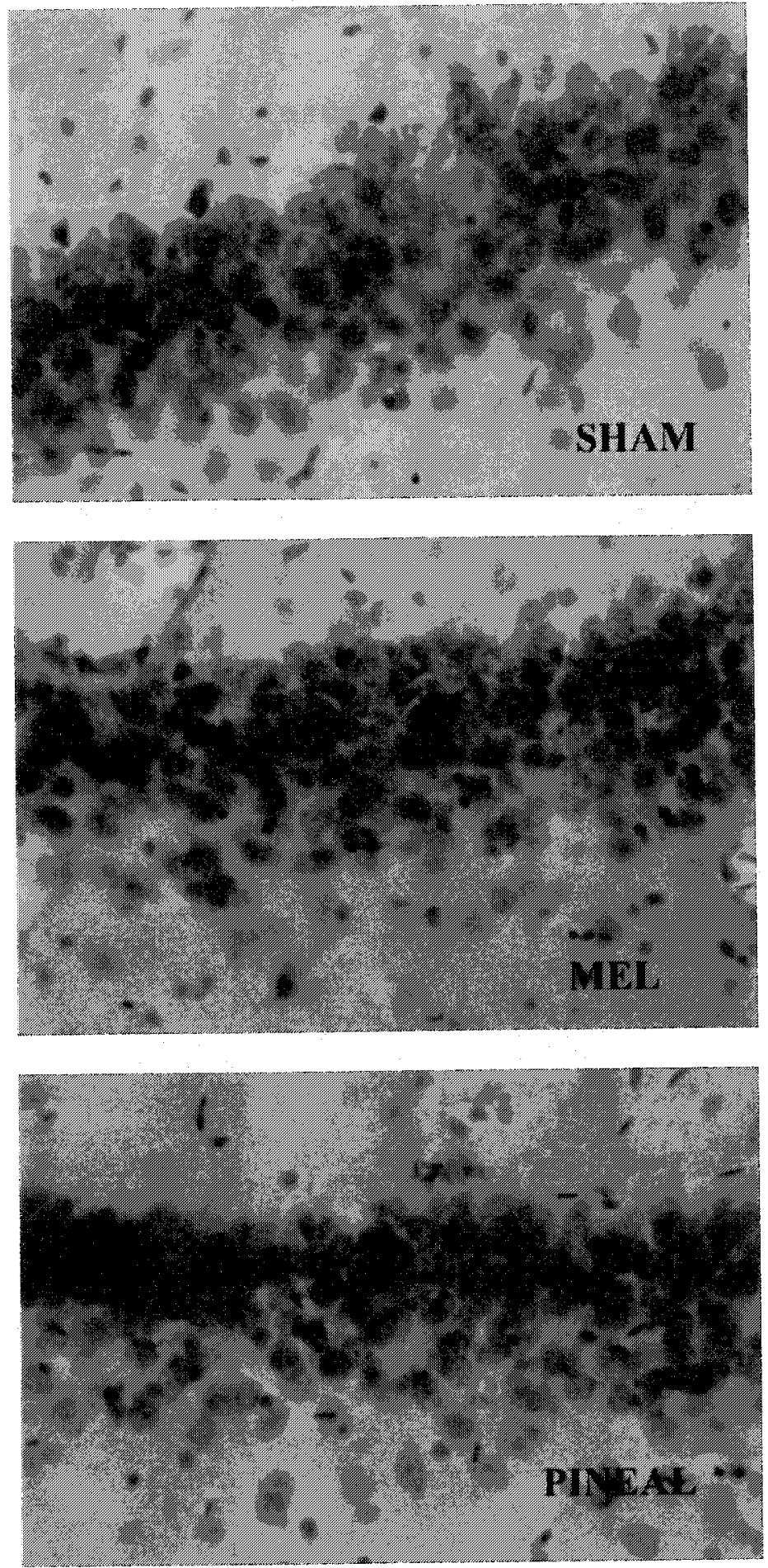
At 6-months post-surgery, an ANOVA revealed that CA1 cell number differed between the groups $(F(2,15)=15.73, p<.0001)$. Specifically, the pinealectomized animals had $15.4 \%$ fewer CA1 cells than the sham (Tukey HSD, $\mathrm{p}<.002$ ) and $18.8 \%$ fewer cells than the melatonin supplemented (Tukey HSD, $\mathrm{p}<.0001$ ).

Similarly, 8-months following surgery, an ANOVA indicated significant group differences in CA1 cell number $(F(2,14)=48.86, p<.0001)$. Pinealectomized animals exhibited a $19.3 \%$ loss of CA1 pyramidal cells compared to the sham animals (Tukey HSD, $p<.0001$ ). They animals also had significantly fewer cells than the melatonin supplemented animals $(\mathrm{p}<.0001)$. Interestingly, melatonin supplemented animals exhibited a significant $10 \%$ increase of cells compared to the sham animals (Tukey HSD, $p<.02)$

At the 10-month post-surgical time-point, a significant difference between the groups was again found $(F(2,11)=18.09, \mathrm{p}<.0001)$. Specifically, pinealectomized animals had $17 \%$ and $19 \%$ fewer cells than the sham (Tukey HSD, $\mathrm{p}<.0002$ ) and melatonin supplemented (Tukey HSD, $\mathrm{p}<.0001$ ) animals respectively. No significant difference in CAl cell number was seen in the melatonin supplemented animals compared to the sham animals.

At the final time-point, a significant difference was found between groups $(F(2,12)=20.67, p<.0001)$. Similar to the previous time-points, melatonin deficient rats exhibited a significant reduction in CA1 cell number compared to the sham $(18 \%$ fewer cells) (Tukey HSD, $\mathrm{p}<.0001)$ and melatonin supplemented rats (19\% fewer cells) (Tukey HSD, $\mathrm{p}<.0001)$. CAl neuron number did not differ between the sham and melatonin supplemented animals. 


\section{CA3 Cell Number}

Similar to the CA1, stereological cell counts of the hippocampal CA3 region were analyzed using one-way ANOVA with group as a between subjects factor. The results for CA3 cell number are shown in Figure 36. Figure 37 is a photomicrograph illustrating CA3 of a sham, melatonin supplemented, and pinealectomized rat. At the 2 -month timepoint, a significant effect of group was found $(F(2,9)=5.66, p<.03)$. A tukey's test revealed the pinealectomized animals had fewer CA3 cell than the sham animals $(17.9 \%$ of sham $)(p<.02)$. No differences were found between the melatonin supplemented rats and the sham animals nor between the melatonin supplemented rats and the pinealectomized rats.

At four months significant differences between groups were found by means of one-way ANOVA $(F(2,15)=7.00, p<.007)$. Specifically, the pinealectomized rats had $18.6 \%$ fewer cells in the CA3 than the shams (Tukey HSD, $\mathrm{p}<.007$ ). Moreover, they rats had $14.4 \%$ fewer CA3 neurons than the melatonin supplemented rats (Tukey HSD, $\mathrm{p}<.05$ ). CA3 cell number did not differ between the sham and melatonin supplemented animals.

At the 6-month time-point, ANOVA revealed significant group differences in CA3 cell number $(F(2,15)=23.50, p<.0001)$. Tukey's test indicated the pinealectomized animals showed a significant loss of $\mathrm{CA} 3$ cells compared to the sham $(\mathrm{p}<.001)$ and melatonin supplemented animals $(\mathrm{p}<.0001)$. The pinealectomized group showed a $19.4 \%$ loss of CA3 neurons compared to the sham group and a $25.6 \%$ loss of cells compared to the melatonin-supplemented group. No differences in CA3 cell number were found between the sham and melatonin supplemented animals. 
Figure 36. Estimated hippocampal CA3 cell counts. Pinealectomy caused a significant reduction in CA3 cells 2 months post-surgery. This was prevented by melatonin supplementation. Error bars represent S.E.M. 


\section{CA3}

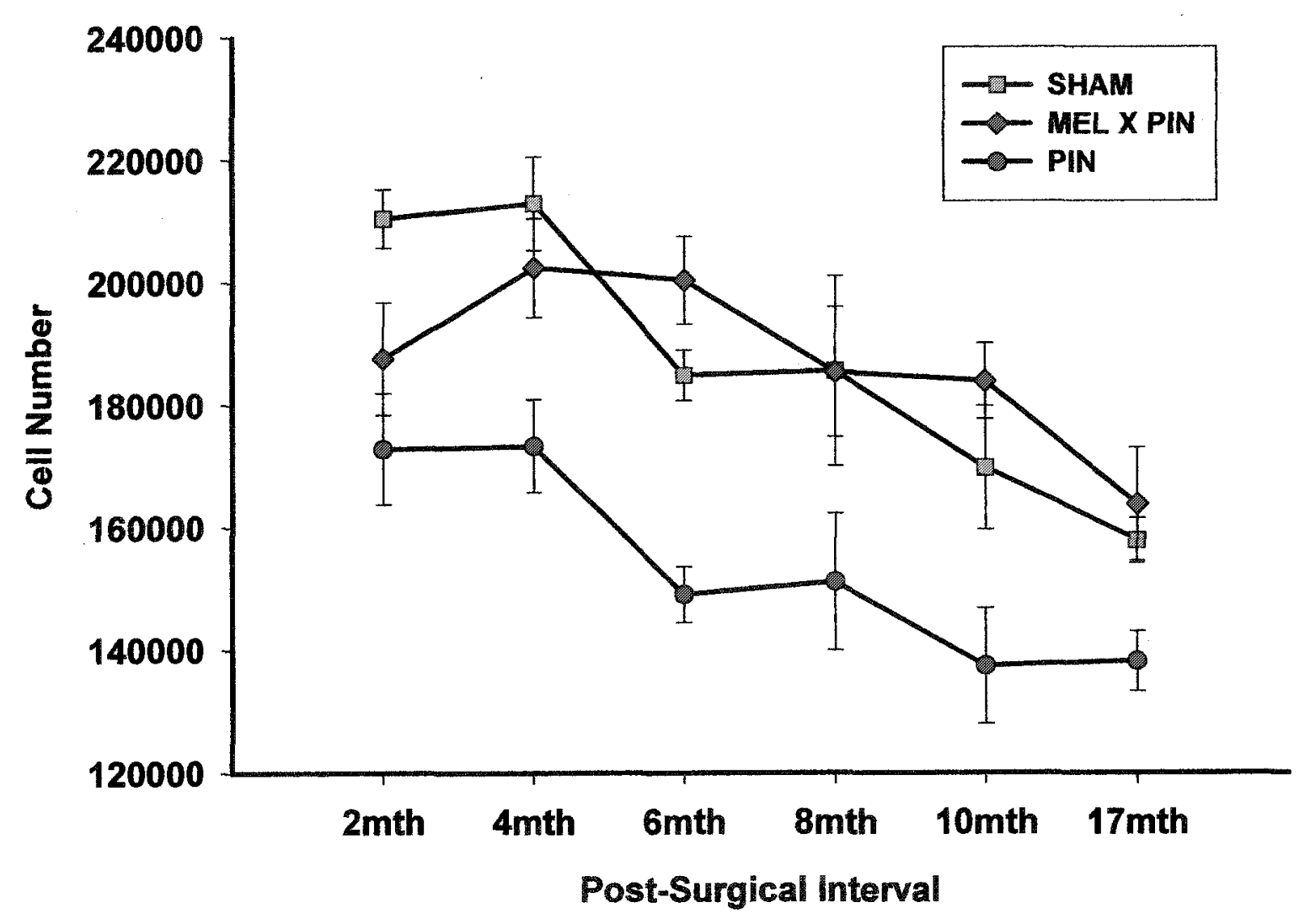


Figure 37. Top Panel: Photomicrograph of CA3 pyramidal cell layer at 20X magnification from a sham rat. Middle Panel: Photomicrograph of CA3 pyramidal cell layer from a melatonin supplemented rat. Lower Panel: Photomicrograph of CA3 pyramidal cell layer from a pinealectomized rat. 

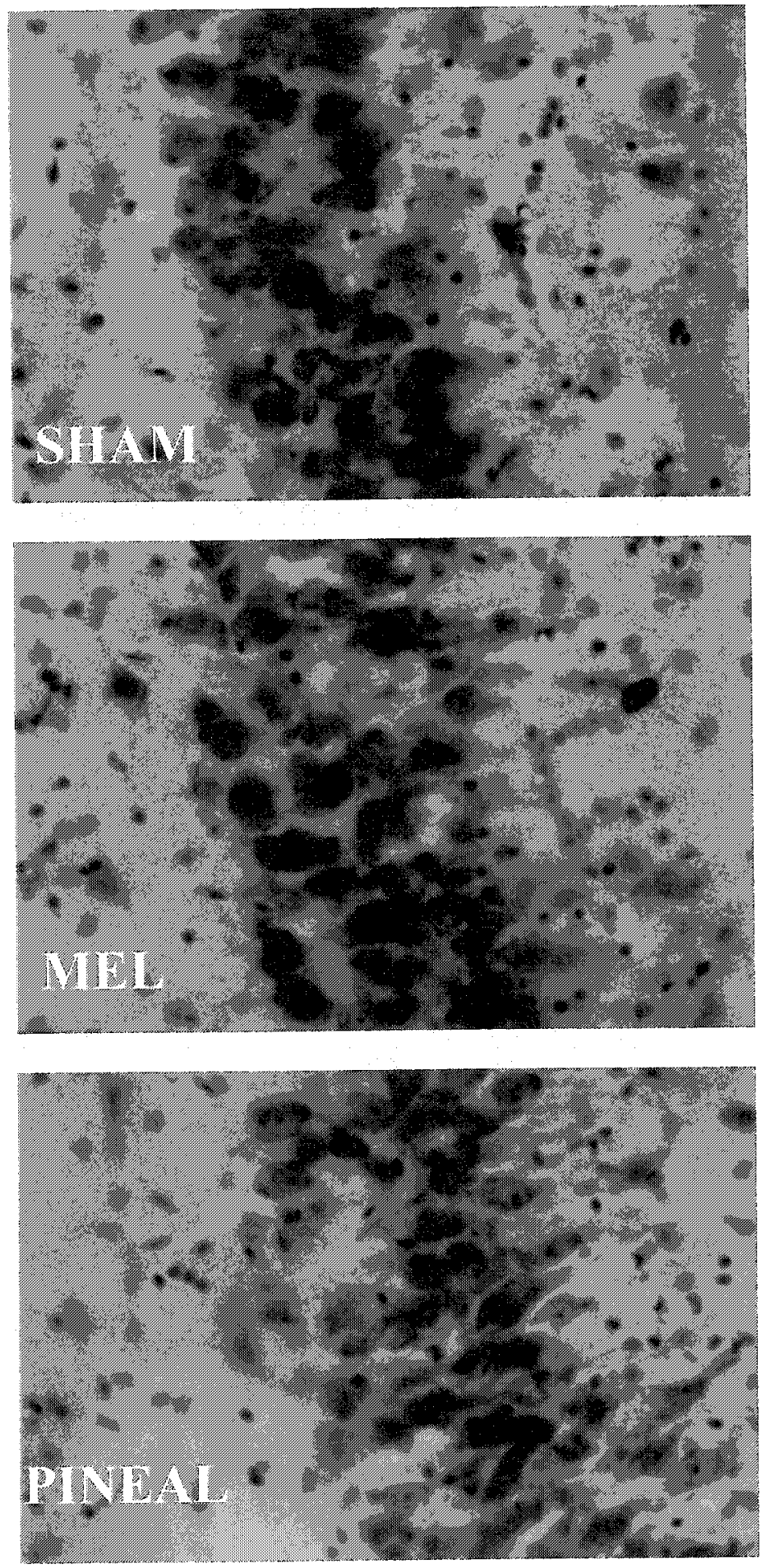
At 8-months post-surgery, ANOVA indicated CA3 cell number differed between the groups $(\mathrm{F}(2,14)=5.22, \mathrm{p}<.02)$. Similar to the previous time-points, pinealectomized rats had the lowest total CA3 cell numbers $(76.1 \%$ of shams, $\mathrm{p}<.02)$. No significant difference in cell number was found between the sham and melatonin-supplemented rats or between the melatonin supplemented and pinealectomized rats.

Ten months following surgery, ANOVA revealed significant differences between the three groups $(F(2,10)=18.09, p<.01)$. Unlike the previous time-points, no significant difference in total CA3 cell number was found between the sham and pinealectomized animals. A significant difference however, was found between the melatonin supplemented and pinealectomized animals (Tukey HSD, $\mathrm{p}<.01$ ). The pinelaectomized rats showed a $25.3 \%$ loss of $\mathrm{CA} 3$ cells compared to rats that received melatonin replacement. No significant difference in cell number was found between the sham and melatonin supplemented animals.

At the 17-month time-point, ANOVA indicated that the groups differed in total CA3 cell number $(F(2,12)=4.38, p<.04)$. Similar to the findings at the 10 -month timepoint, pinealectomized animals showed a $15.6 \%$ reduction in CA3 cells compared to the melatonin supplemented animals $(\mathrm{p}<.04)$. No significant differences in cell number were found between the sham and melatonin supplemented animals nor between the sham and pinealectomized animals.

\section{Effect of Age on CAI and CA3 Cell Number}

In order to determine whether there was a significant effect of age on hippocampal CA1 and CA3 cell number, one-way ANOVAs with age $(2,4,6,8,10$, and 
17 months) as between subjects factor, were conducted for each of the three groups. In the sham group, ANOVA revealed a significant effect of age for both the CA1 $(F(5,29)=$ $3.43, \mathrm{p}<.02)$ and CA3 $(\mathrm{F}(5,24)=9.66, \mathrm{p}<.0001)$ regions. Tukey's test revealed a significant difference in CAl cell number between the 4-month old sham animals compared to the 17-month old sham animals. Specifically, 17-month old rats had a $12 \%$ reduction of cells compared to the 4-month old sham rats $(p<.02)$. No significant differences were found between any of the other age groups.

In addition, Tukey's test revealed a significant reduction of pyramidal CA3 cells in the 10 and $17-$ month animals compared to the 2 and 4 month animals. Specifically, the 10 month old sham rats had $19 \%$ fewer cells in the CA3 compared to the 2 month old rats and $20 \%$ fewer cells than the 4 month old sham rats $(p<.02)$. Moreover, 17 -month old sham rats had $25 \%$ fewer CA3 cells than the 2 month old rats and $26 \%$ fewer CA3 cells than the 4 month old sham animals $(p<.001)$. Animals in the 17-month group also had $21 \%$ fewer CA3 cells compared to the 8 month old animals.

In the pinealectomy age groups, a significant effect of age was found for both the $\mathrm{CA} 1(\mathrm{~F}(5,26)=6.99, \mathrm{p}<.0001)$ and CA3 $(\mathrm{F}(5,26)=3.61, \mathrm{p}<.01)$ sectors. Tukey's test revealed that 4-month pinealectomized rats had $19 \%$ fewer CA1 cells compared to the 2month rats $(\mathrm{p}<.001)$. Moreover, at 17 - months post-pinealectomy, rats had $22 \%$ fewer CAl cells compared to the 2 -month old rats $(p<.0001)$. Both 8 and 10 month pinealectomized rats also had significantly fewer CA1 cells compared to the 2-month rats $(p<.01)$

For the hippocampal CA3 region, significant differences in cell number were observed between the 4-month group of pinealectomized animals and the 10 and 17 - 
month animals. Specifically, 10-month pinealectomized animals had $21 \%$ fewer CA3 cells compared to the 4-month animals while the 17-month old animals had $20 \%$ fewer cells.

Although a significant effect of age was found between the different melatonin supplemented age groups for the $\mathrm{CAl}(\mathrm{F}(5,31)=10.66, \mathrm{p}<.0001)$ region, a different pattern of results was found for them compared to the sham and pinealectomized age groups. Tukey's test revealed that 6-month melatonin supplemented animals had $13 \%$ and $17 \%$ significantly more $\mathrm{CA} 1$ cells compared to the 2 and 4 -month animals respectively $(\mathrm{p}<.01)$. Moreover, 8 -month melatonin supplemented animals had $19 \%$ and $23 \%$ more CA1 cells compared to the 2 and 4-month animal respectively $(\mathrm{p}<.0001)$.

In addition, the 10-month melatonin supplemented animals had $14 \%$ fewer CAl cells compared to the 4-month melatonin supplemented rats (Tukey HSD, $\mathrm{p}<.04$ ). A significant $18 \%$ reduction of $\mathrm{CA} 1$ cells was also found in the 17-month group compared to the 8-month old animals (Tukey HSD, $\mathrm{p}<.001$ ).

A significant effect of age was also found for the hippocampal CA3 $(F(5,25)=$ $2.60, \mathrm{p}<.05$ ) region. Melatonin supplemented rats in the 17 -month group had a significant $19 \%$ reduction of CA3 cells compared to the 4-month rats. No other differences in CA3 cell number were found between the melatonin supplemented age groups.

\section{Fluoro-Jade B Staining}

A set of three tissue sections containing the hippocampus were stained with Fluoro-Jade B. Hippocampal cell counts were carried out by manually counting the number of fluorescent stained cells in the $\mathrm{CA} 1, \mathrm{CA} 3, \mathrm{CA} 4$, and dentate gyrus regions. 
Results were analyzed by an ANOVA with group (sham, melatonin supplemented, and pinealectomized) and age ( 3 and 20 months) as between subjects factors. Post-hoc comparisons were performed using Tukey's test.

Figures $38,39,40$ and 41 illustrate the results of the Fluoro-Jade B cell counts. The ANOVAs did not indicate significant group differences in any area at any of the time-points examined. A significant main effect of age however, was found for the dendate gyrus region of the hippocampus $(F(5,54)=2.37, \mathrm{p}<.05)$. Tukey's test indicated significantly higher staining in the 4-month old animals compared to the 6-month old animals $(p<.03)$. This effect narrowly missed significance for both CA1 and CA4 sectors of the hippocampus $(p=.07)$.

An ANOVA on a compilation of all the Fluoro-Jade B data from all hippocampal regions, revealed a significant effect of age $(F(5,270)=7.18, p<.0001)$ and a significant Age X Group interaction $(F(10,270)=3.79, \mathrm{p}<.0001)$. Tukey's test indicated significantly higher staining in the 4-months animals compared to the 6 -months animals $(p<.0001)$. Moreover, Tukey's test indicated that melatonin supplemented rats exhibited significantly fewer positively stained cells compared to the pinealectomized rats at the 4-month timepoint $(p<.05)$. This is illustrated in Figure 42.

\section{Biochemistry}

TBARS Levels

\section{Months}

Tissue samples of the hippocampus were collected for each of the three groups and TBARS levels were determined. The data represent the nmol of TBARS/g wet tissue. 
Figure 38. Hippocampal CA1 Fluoro-Jade B cell counts. The groups did not differ in the number of CA1 cells that showed positive staining at any of the post-surgical intervals. Error bars represent S.E.M. 
Fluoro-Jade Staining in CA1

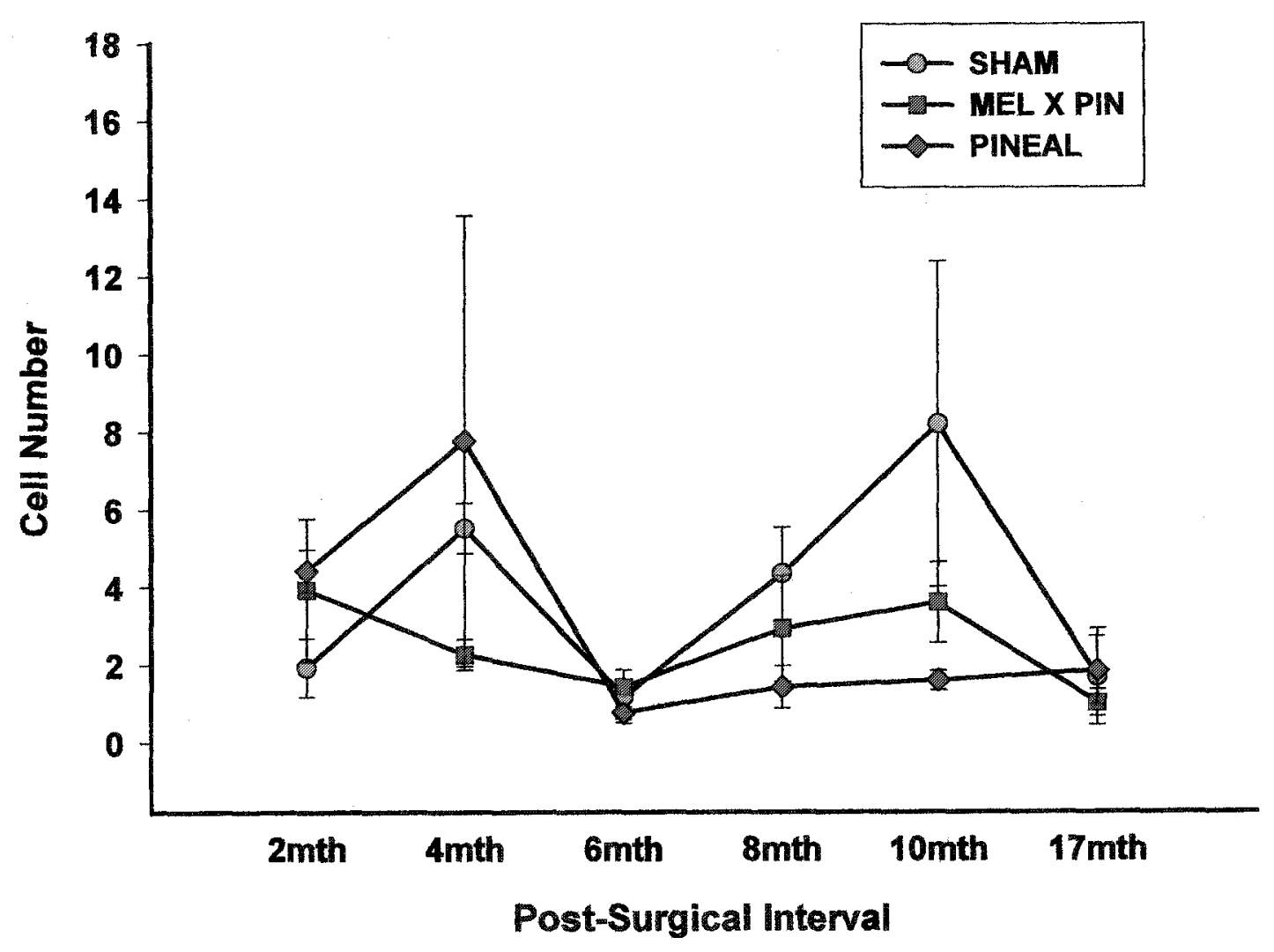


Figure 39. Hippocampal CA3 Fluoro-Jade B cell counts. The groups did not differ in the number of $\mathrm{CA} 3$ cells that showed positive staining at any of the post-surgical intervals. Error bars represent S.E.M. 


\section{Fluoro-Jade Staining in CA3}

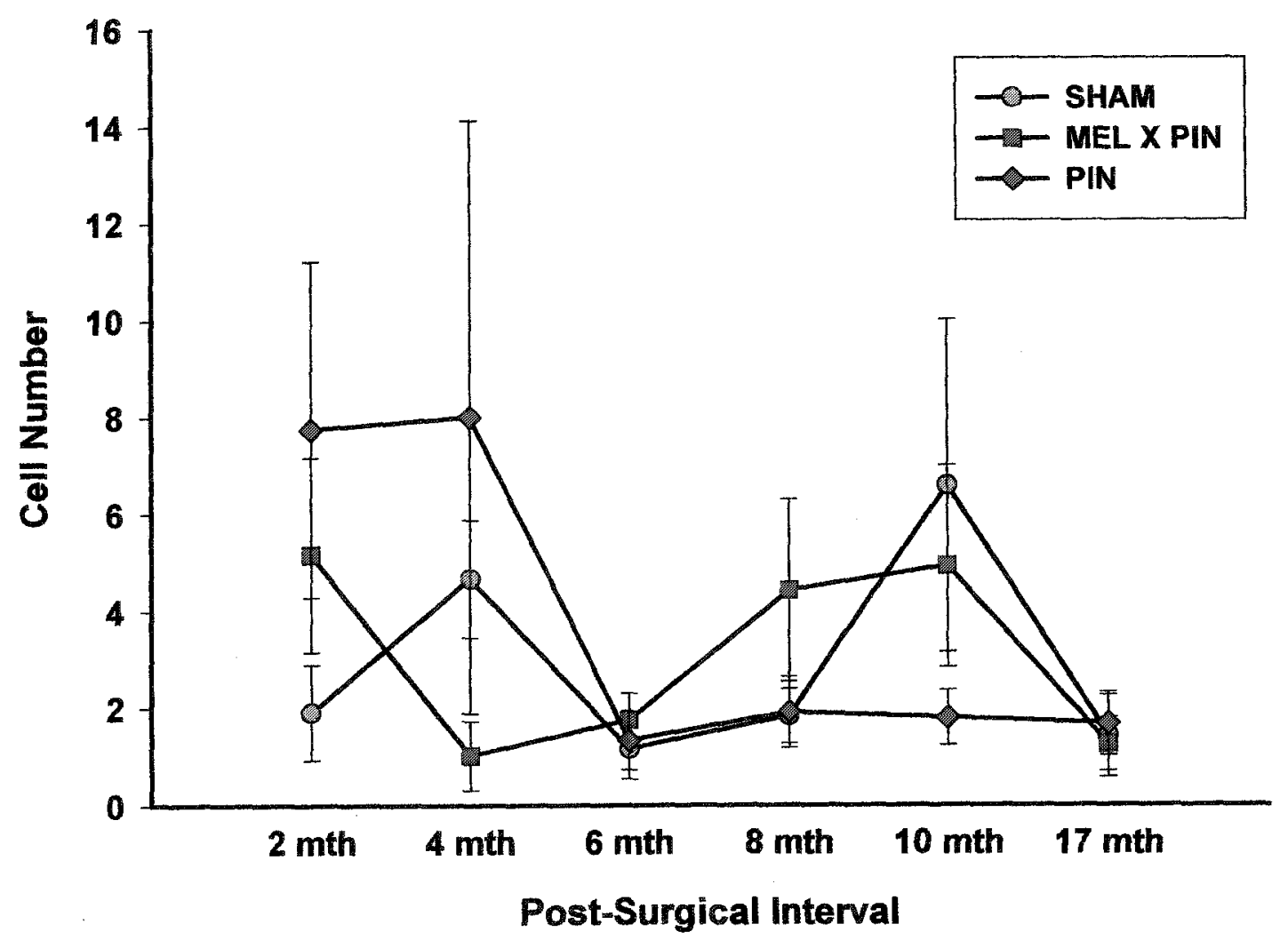


Figure 40. Hippocampal CA4 Fluoro-Jade B cell counts. No significant group differences were found in the number of CA4 cells that showed positive staining at any of the time-points examined. Error bars represent S.E.M. 
Fluoro-Jade Staining in CA4

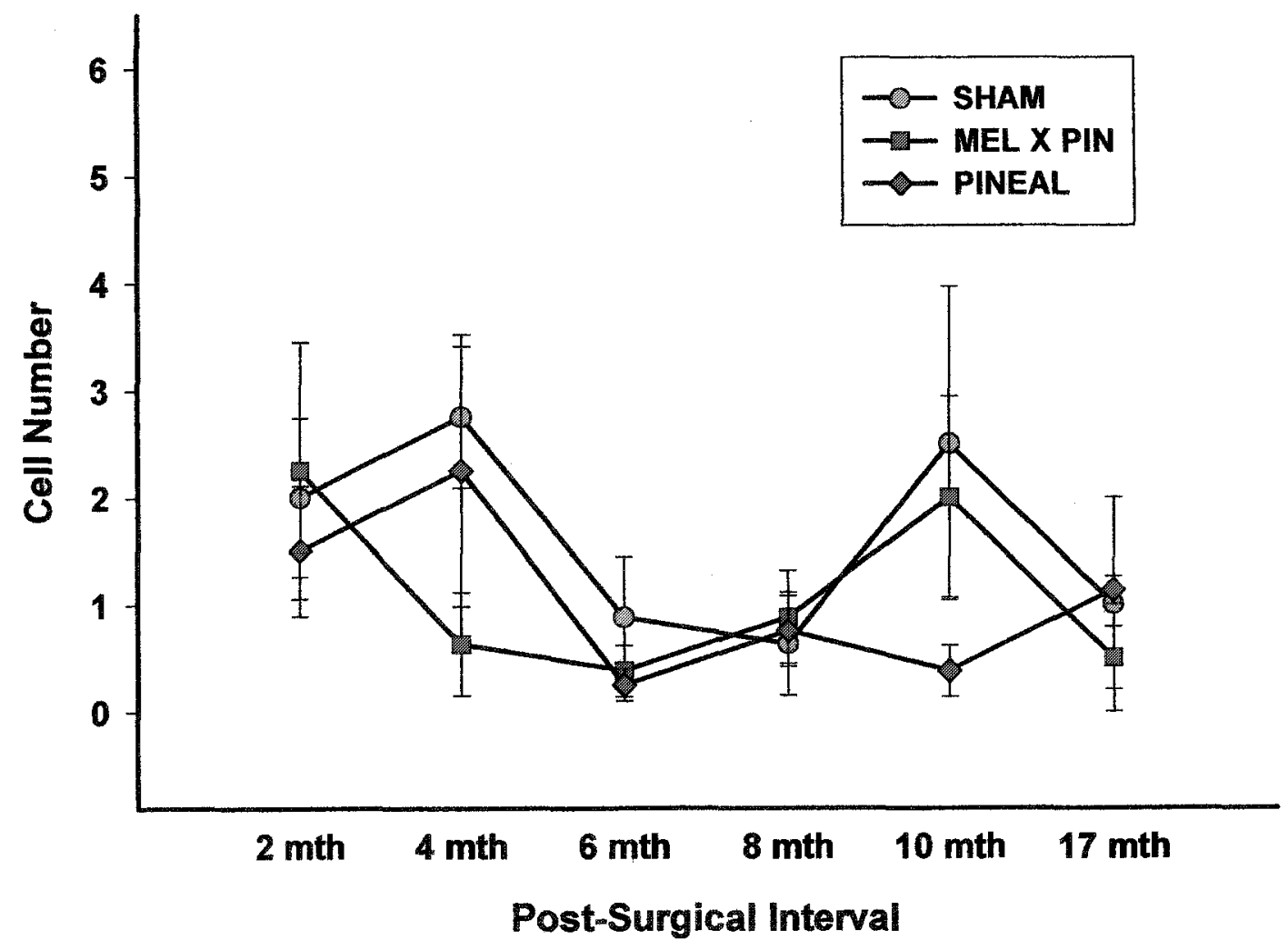


Figure 41. Fluoro-Jade B cell counts in dentate gyrus. The number of fluorescent cells did not differ between the three groups at any of the post-surgical intervals. Error bars represent S.E.M. 


\section{Fluoro-Jade Staining in Dentate Gyrus}

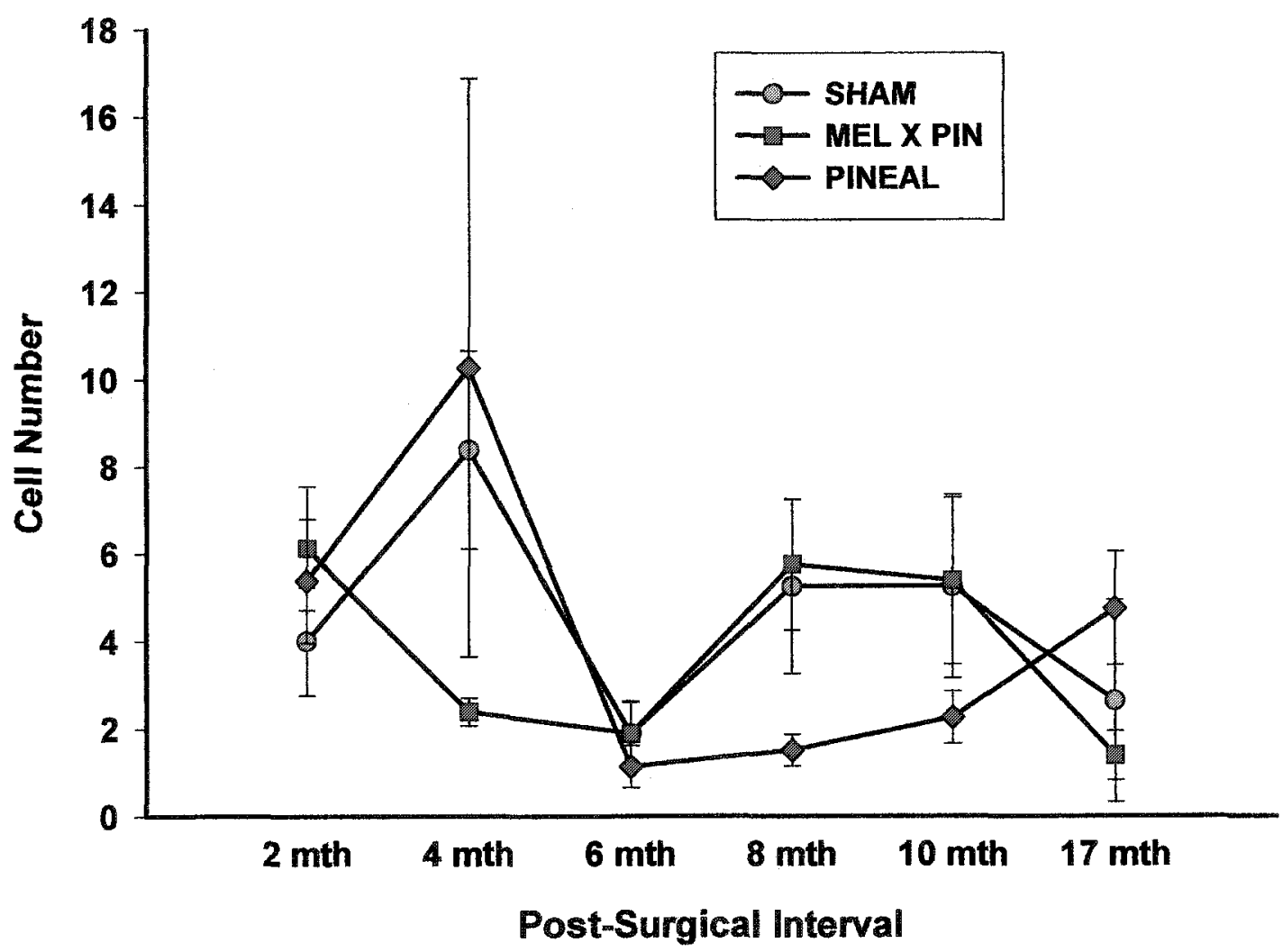


Figure 42. Hippocampal Fluoro-Jade B cell counts. Melatonin supplemented rats exhibited significantly fewer positively stained cells 4 months post-surgery compared to the pinealectomized rats $(\mathrm{p}<.05)$. Error bars represent S.E.M. 


\section{Hippocampal Fluoro-Jade B Staining}

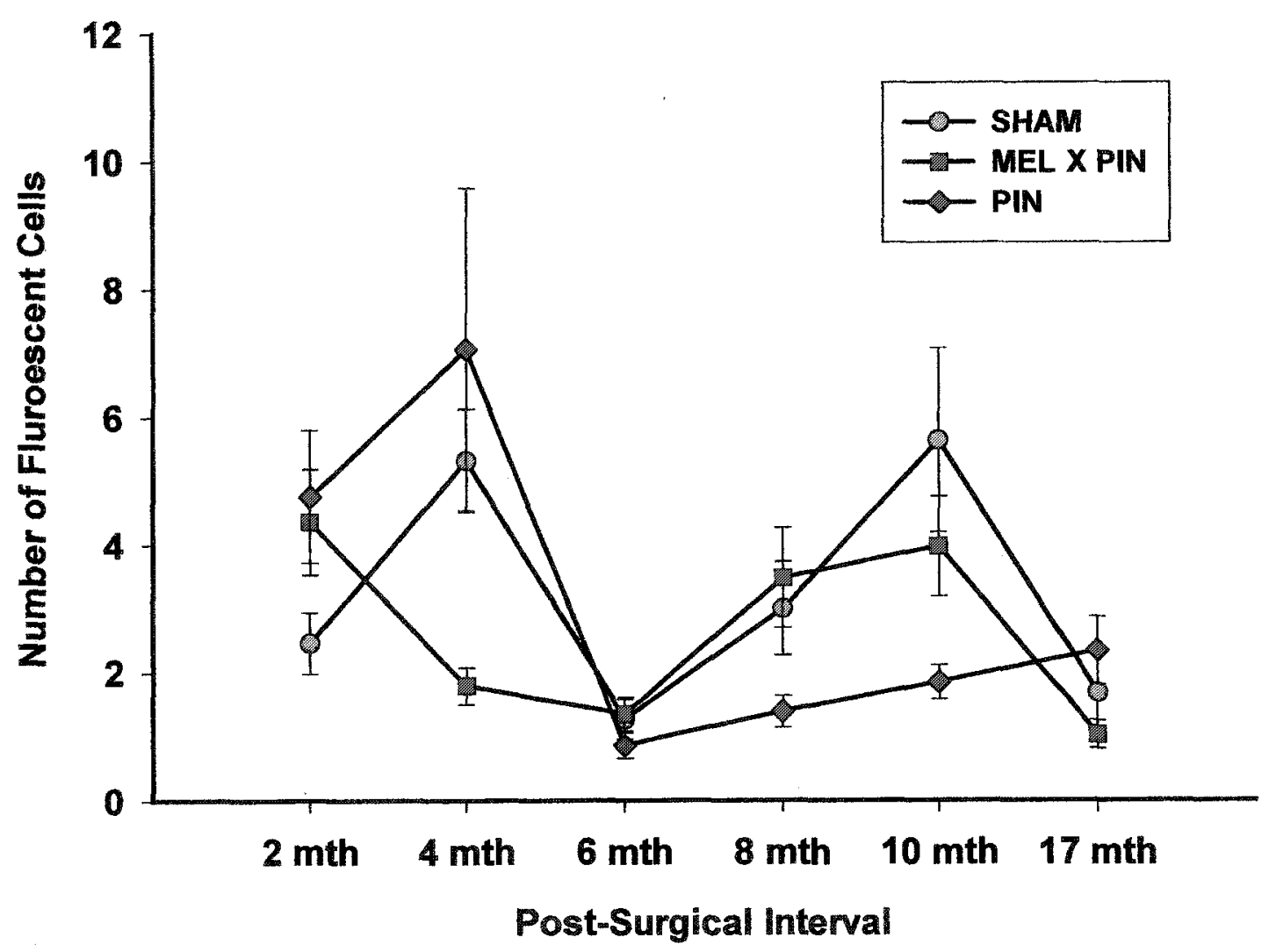


The data were analyzed by means of a one-way ANOVA with group (sham, melatonin supplemented, and pinealectomized) as a between subjects factor. Post-hoc comparisons were performed using Tukey's test.

As shown in Table 2, no significant differences in hippocampal TBARS levels were found between the sham, melatonin supplemented, and pinealectomized rats.

\section{Months}

Tissue samples of the cortex, hippocampus, and kidney were collected for each of the three groups and TBARS levels were determined. The data represent the nmol of TBARS/g wet tissue. The data were analyzed by means of a one-way ANOVA with group (sham, melatonin-supplemented, and pinealectomized) and region (cortex, hippocampus, and kidney) as between- subjects factors. Post-hoc comparisons were performed using Tukey's test. As shown in Table 3, no significant differences were found between the groups on hippocampal, cortical, or kidney TBARS levels. ANOVA did indicate however, a significant effect of region $(F(2,60)=17.95, p<.0001)$. Tukey's test revealed that TBARS levels were significantly higher in the kidney compared to the hippocampal $(p<.0001)$ and cortical $(p<.001)$ brain regions. No Region X Group interaction effect was observed indicating that all groups exhibited higher TBARS levels in the kidney compared to the brain regions.

\section{Effect of Age on Hippocampal TBARS Levels}

One-way ANOVA with group (sham, melatonin supplemented, and pinealectomized) and age ( 3 and 20 month) as between-subjects factors, revealed a 
Table 2. TBARS levels (nmol/g wet tissue) in the hippocampus of 3-month sham, melatonin supplemented, and pinealectomized rats. No significant group differences were found. Data are means \pm S.E.M.

\begin{tabular}{|c|c|c|c|}
\hline Region & Sham & Pin X Mel & Pineal \\
\hline Hippocampus & $7.97 \pm .73$ & $7.16 \pm 1.06$ & $7.55 \pm 1.18$ \\
\hline
\end{tabular}


Table 3. TBARS levels (nmol/g wet tissue) in the cortex, hippocampus, and kidney of 20-month sham, melatonin supplemented and pinealectomized rats. No significant group differences were found. Data are means \pm S.E.M.

\begin{tabular}{|c|c|c|c|}
\hline Group & Cortex & Hippocampus & Kidney \\
\hline Sham & $11.76 \pm 1.06$ & $10.59 \pm .83$ & $16.88 \pm 1.64$ \\
\hline Pin X Mel & $11.97 \pm 1.30$ & $9.32 \pm .96$ & $16.50 \pm 1.84$ \\
\hline Pineal & $11.66 \pm .87$ & $8.80 \pm .97$ & $14.57 \pm 1.94$ \\
\hline
\end{tabular}


significant effect of age on hippocampal TBARS levels $(F(1,36)=6.32, p<.02)$. As

illustrated in Figure 43, significantly higher TBARS levels were found in the hippocampus of the 20 -month old rats compared to the 3 -month old rats. No significant Group effect was found nor was there a significant Group X Age effect.

\section{Glutathione Levels}

GSH and GSSG levels at 3-Months of Age

Tissue samples of the hippocampus were collected for each of the three groups. GSH and GSSG concentrations and the ratio of GSH to GSSG were determined. Data was presented as pg of GSH or GSSG/ ug of protein. The data were analyzed by means of a one-way multivariate ANOVA with group (sham, melatonin supplemented, and pinealectomized) as a between-subjects factor and GSH, GSSG, and GSH/GSSG ratio as dependant variables. Post-hoc comparisons were performed using Tukey's Test.

ANOVA revealed a significant effect of Group for the ratio of GSH to GSSG $(\mathrm{F}(2,17)=5.11, \mathrm{p}<.02)$.(Figure 44). Tukey's test indicated that the GSH/GSSG ratio was increased in the hippocampus of pinealectomized animals compared to the sham and melatonin supplemented animals $(p<.04$ and $p<.03$ respectively). As shown in Figure 45 , no significant differences were found between the groups for GSH or GSSG levels.

\section{GSH and GSSG levels at 20-Months of Age}

Tissue samples of the hippocampus were collected for each of the three groups. Both GSH and GSSG levels were determined as well as the ratio of GSH to GSSG. The data were analyzed by means of a one-way multivariate ANOVA with group (sham, 
Figure 43. Age effects on hippocampal TBARS levels. Aged rats demonstrated significantly higher hippocampal TBARS levels compared to the young rats $(F(1,36)=$ $6.32, \mathrm{p}<.02)$. Error bars represent S.E.M. 
TBARS Levels

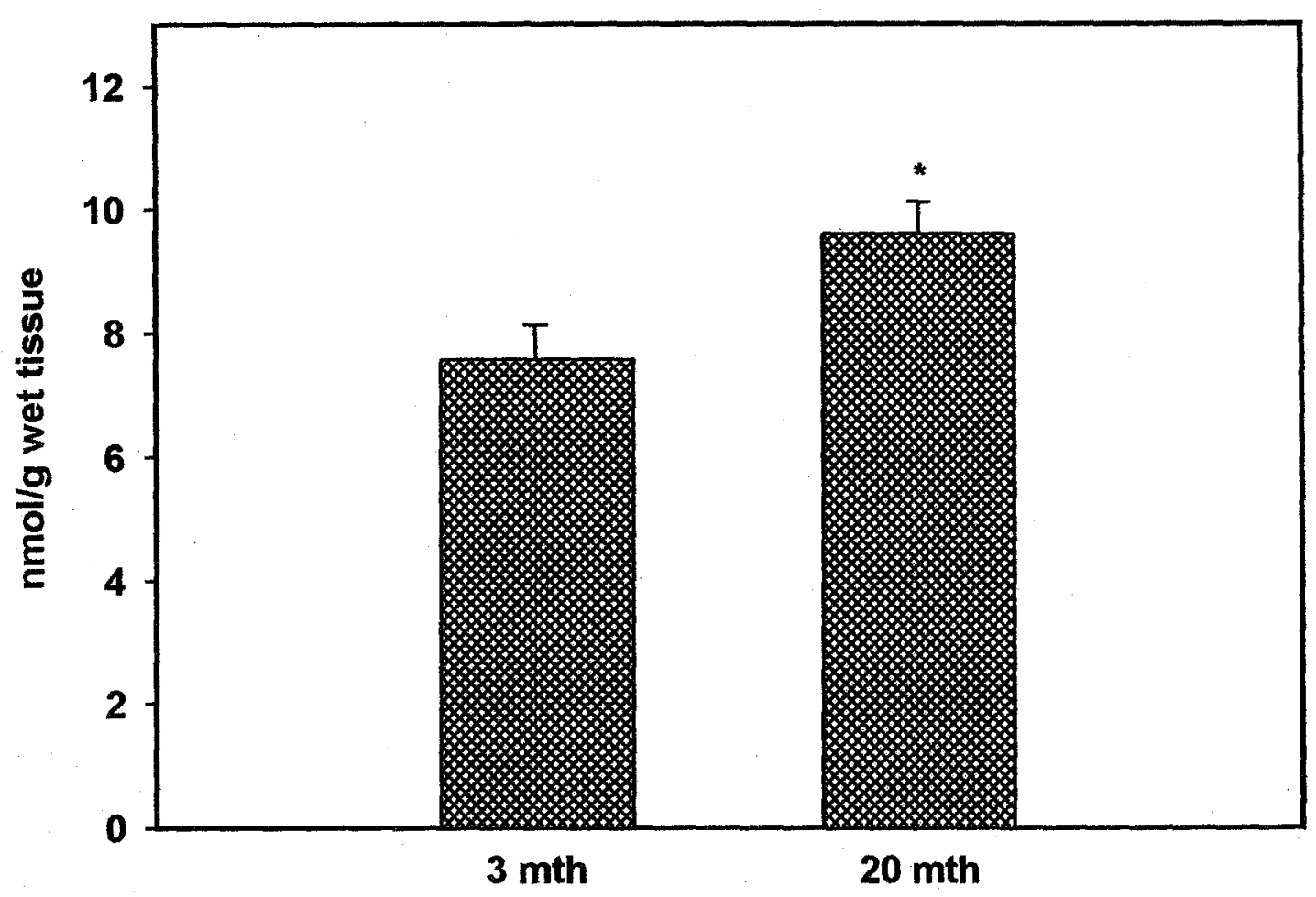


Figure 44. Ratio of GSH to GSSG at 3-months post-surgery. The ratio of GSH to GSSG was significantly elevated in the pinealectomized rats compared to the sham and melatonin supplemented rats $(\mathrm{F}(2,17)=5.11, \mathrm{p}<.02)$. Error bars represent S.E.M. 
Hippocampal GSH/GSSG Ratio

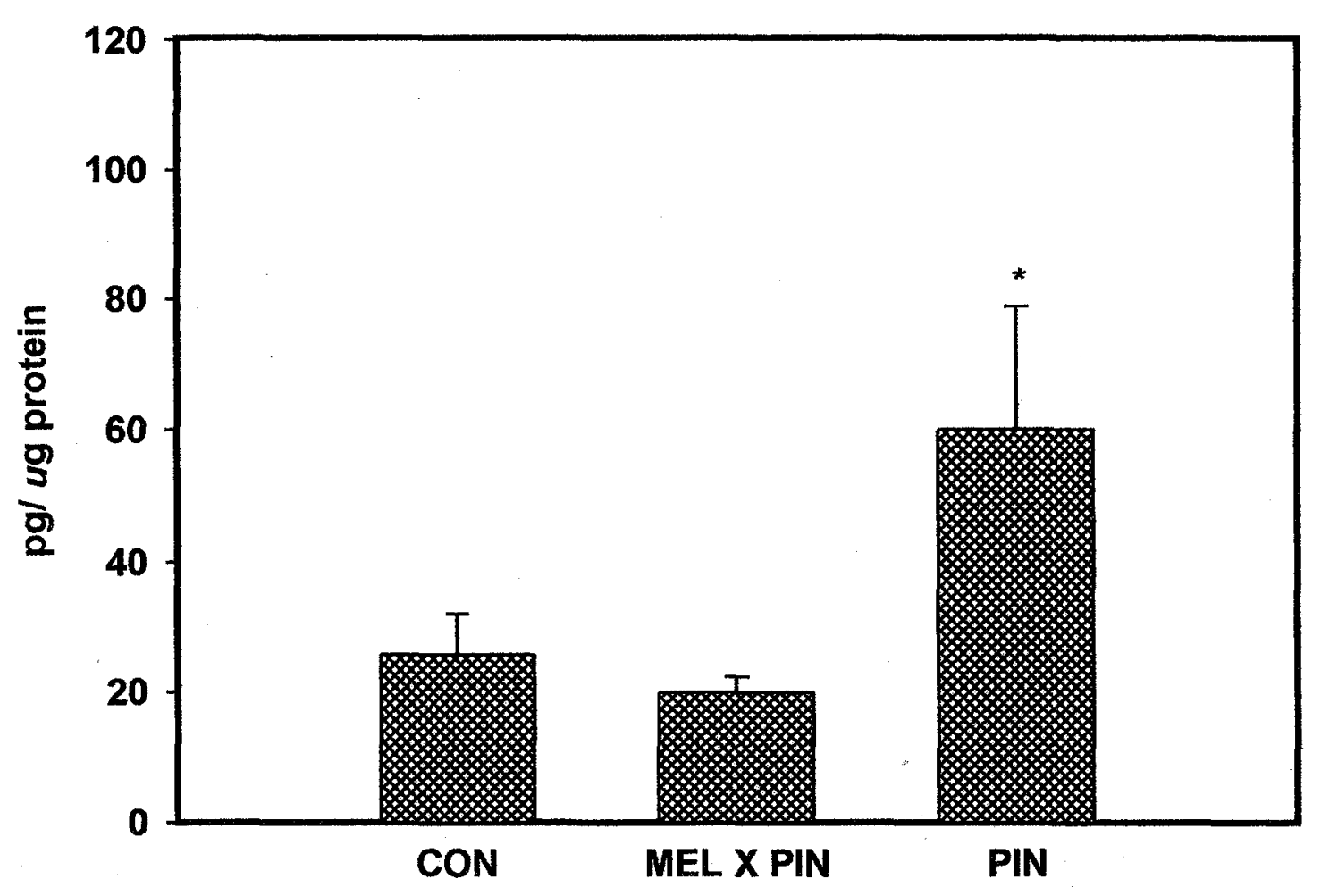


Figure 45. Top Panel: GSH levels at 3-months post-surgery. No significant group differences were found in reduced glutathione levels. Bottom Panel: GSSG levels at 3months post-surgery. No group differences were found in oxidized glutathione levels. Error bars represent S.E.M. 
Hippocampal GSH Levels

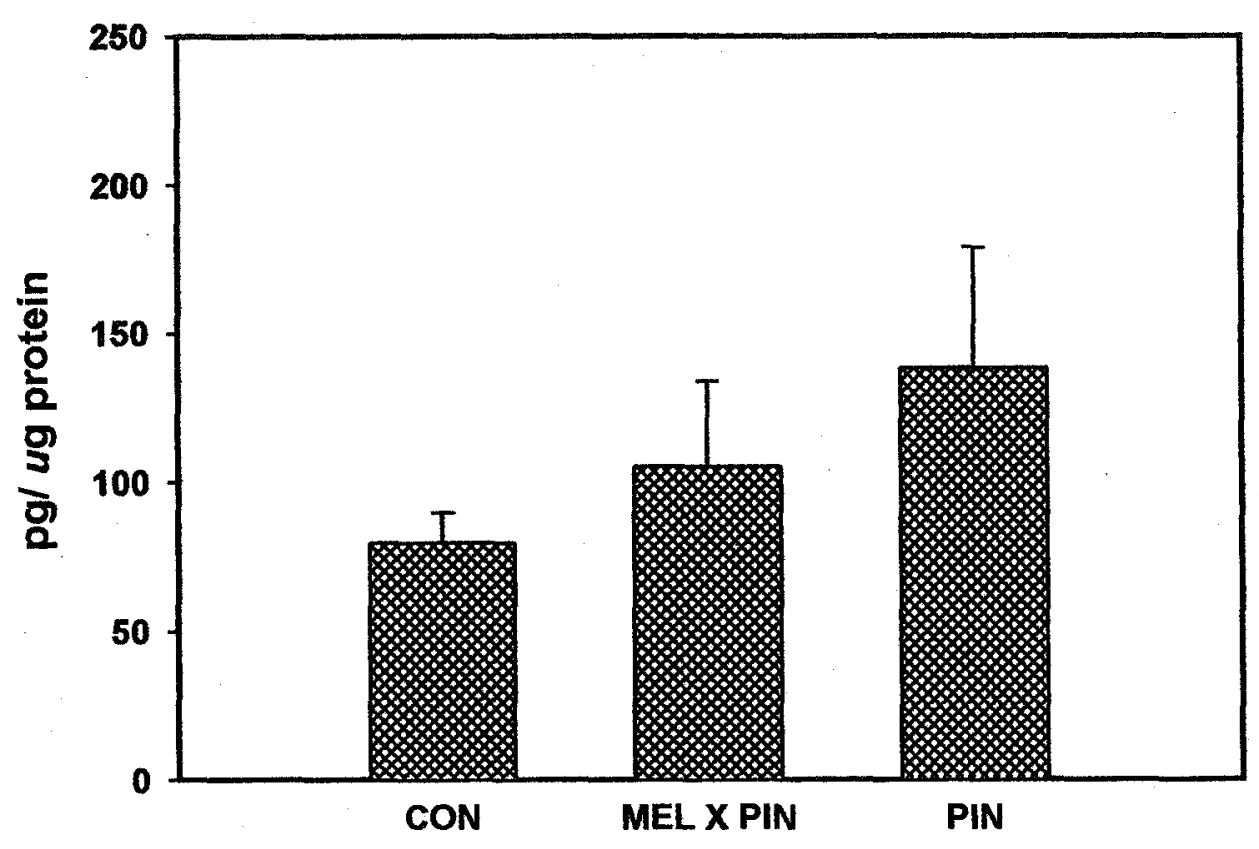

Hippocampal GSSG Levels

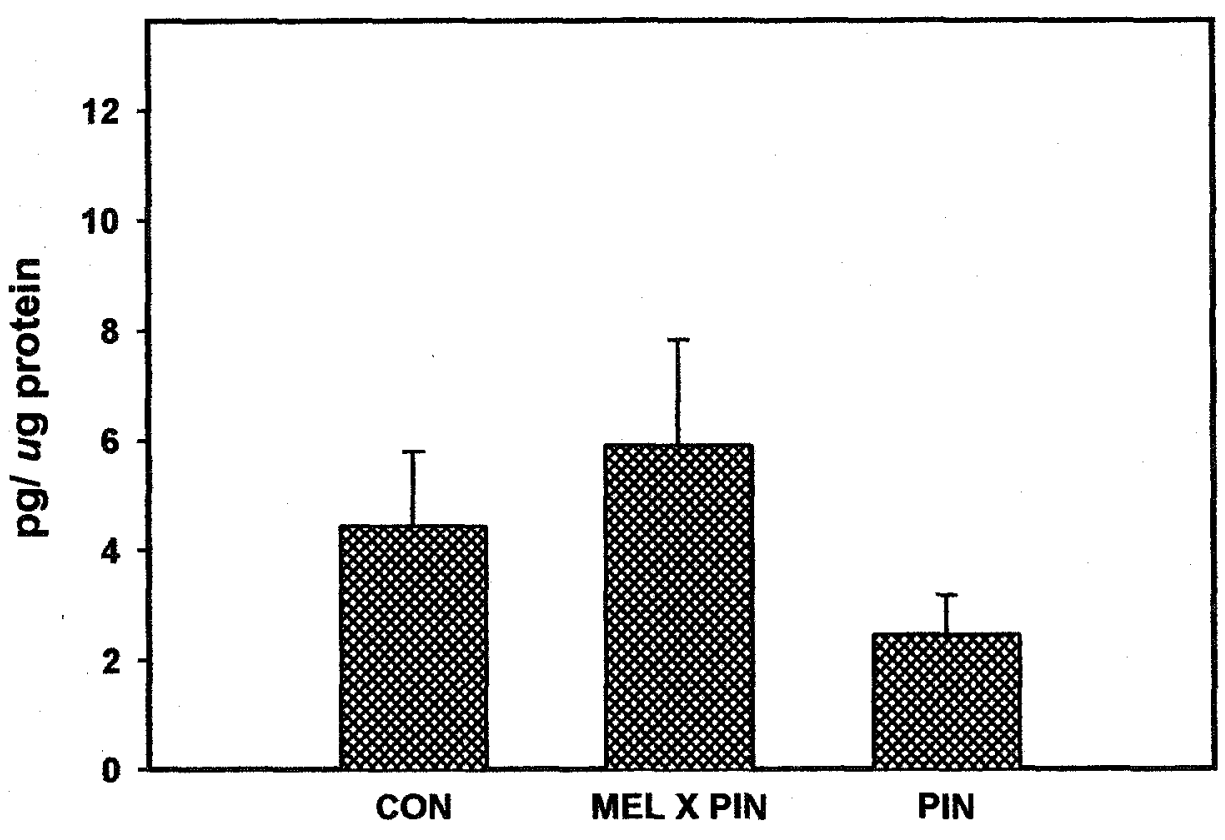


melatonin supplemented, and pinealectomized) as a between-subjects factor and GSH, GSSG, and GSH/GSSG as dependent variables. Post-hoc comparisons were performed using Tukey's test.

As shown in Figure 46, no significant differences were found in GSH or GSSG levels between the groups. Moreover, the groups did not differ on the GSH/GSSG ratio (Figure 47).

\section{Effect of Age on GSH and GSSG Concentrations}

A one-way multivariate ANOVA with group (sham, melatonin supplemented, and pinealectomized) and age ( 3 months and 20 months) as between- subjects factors and GSH, GSSG, and GSH/GSSG ratio as dependent variables was performed. The results are shown in Table 4. ANOVA revealed a significant effect of age on hippocampal GSH concentrations $(F(1,37)=5.4, p<.03)$. Specifically, 3-month-old rats exhibited significantly higher GSH levels compared to the 20 -month-old rats. No significant main effect of group was found nor was there a significant Age X Group interaction for GSH level. No significant age effects were found for GSSG levels.

A significant age $(F(1,37)=11.51 ; p<.002)$ and group $(F(2,37)=6.13 ; p<.01)$

main effect was found for the ratio of GSH to GSSG. As well, a significant Age X Group interaction was found for this measure $(F(2,37)=4.79, p<.02)$. Specifically, 3 -month pinealectomized rats had a significantly higher GSH to GSSG ratio compared to the 20month pinealectomized rats. This effect was not observed in the sham and melatonin supplemented groups. 
Figure 46. Top Panel: GSH levels at 20-months post-surgery. No significant differences were found in reduced glutathione levels. Bottom Panel: GSSG levels. No significant differences were found in oxidized glutathione levels. Error bars represent S.E.M. 
Hippocampal GSH Levels

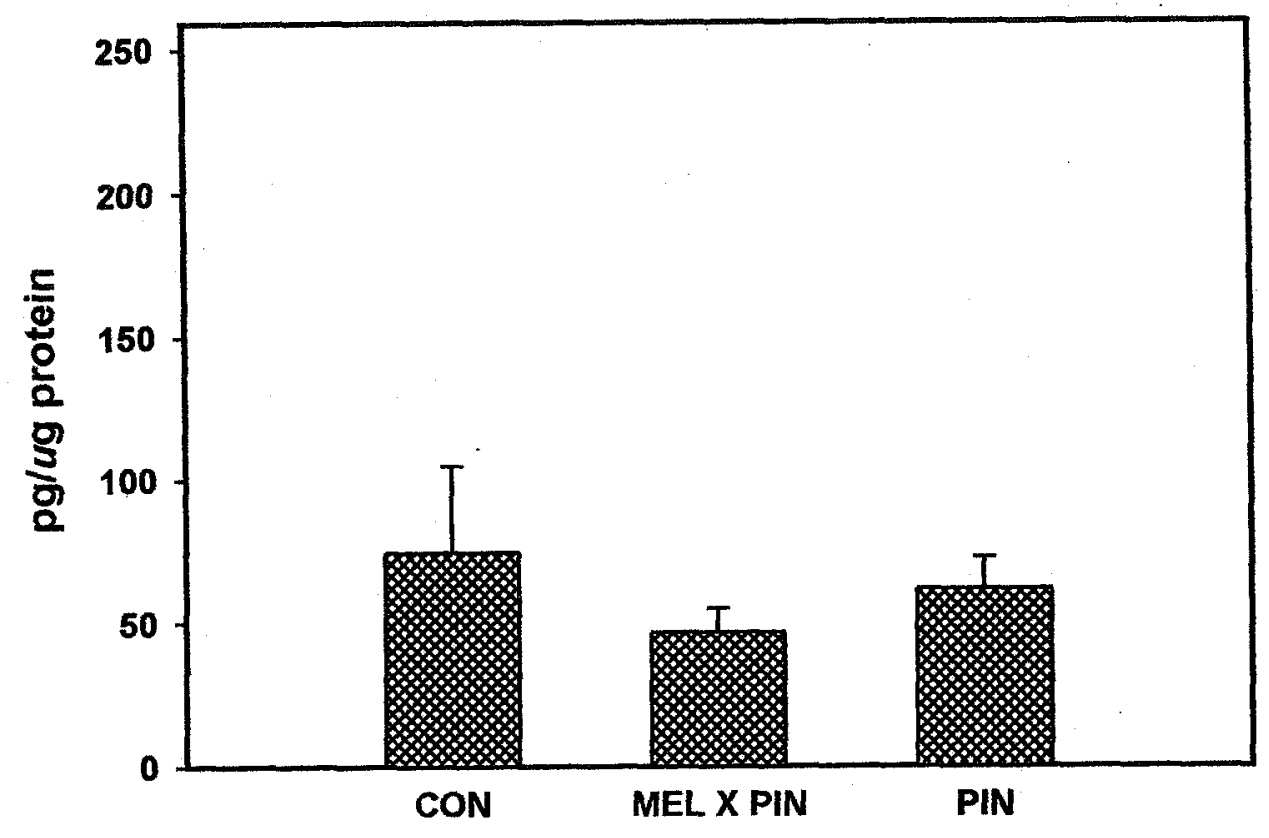

Hippocampal GSSG Levels

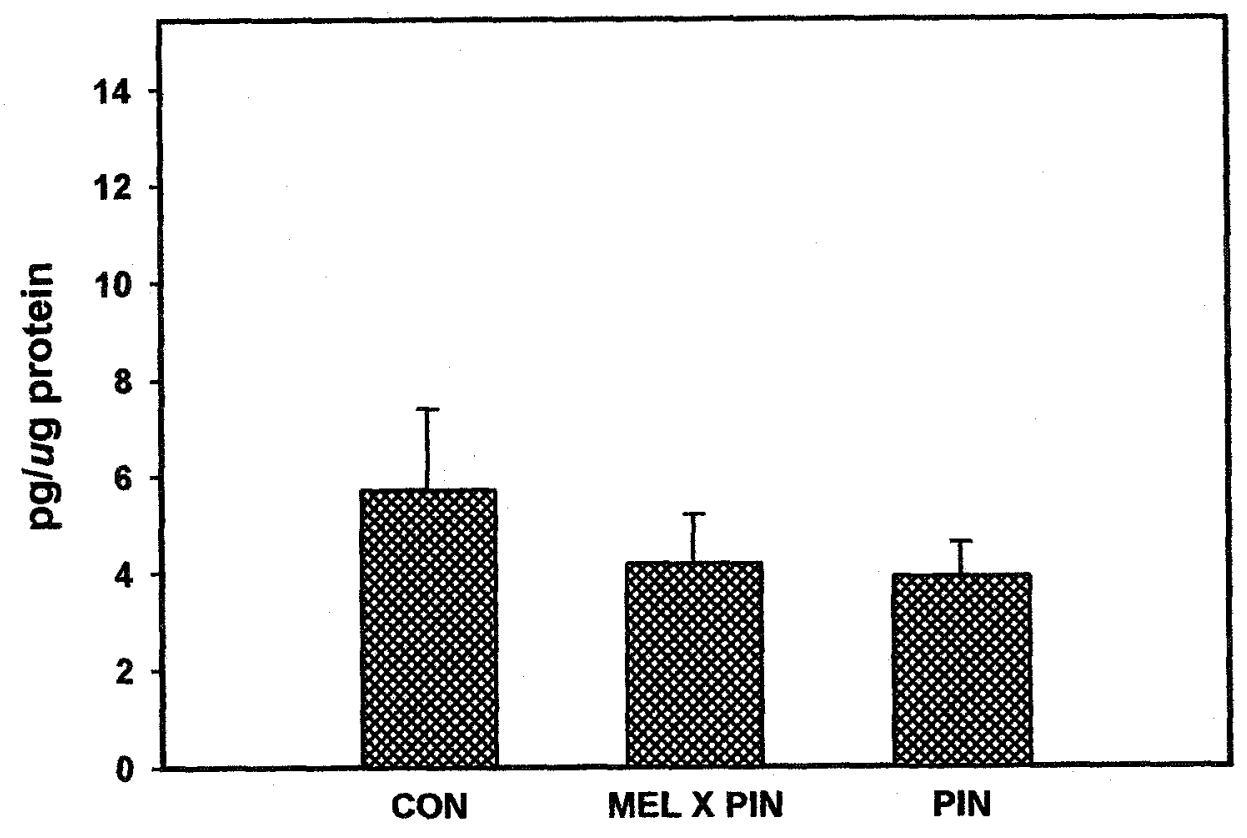


Figure 47. Ratio of GSH to GSSG at 20-months post-surgery. No significant differences were found between the three groups. Error bars represent S.E.M. 
GSH/GSSG Ratio

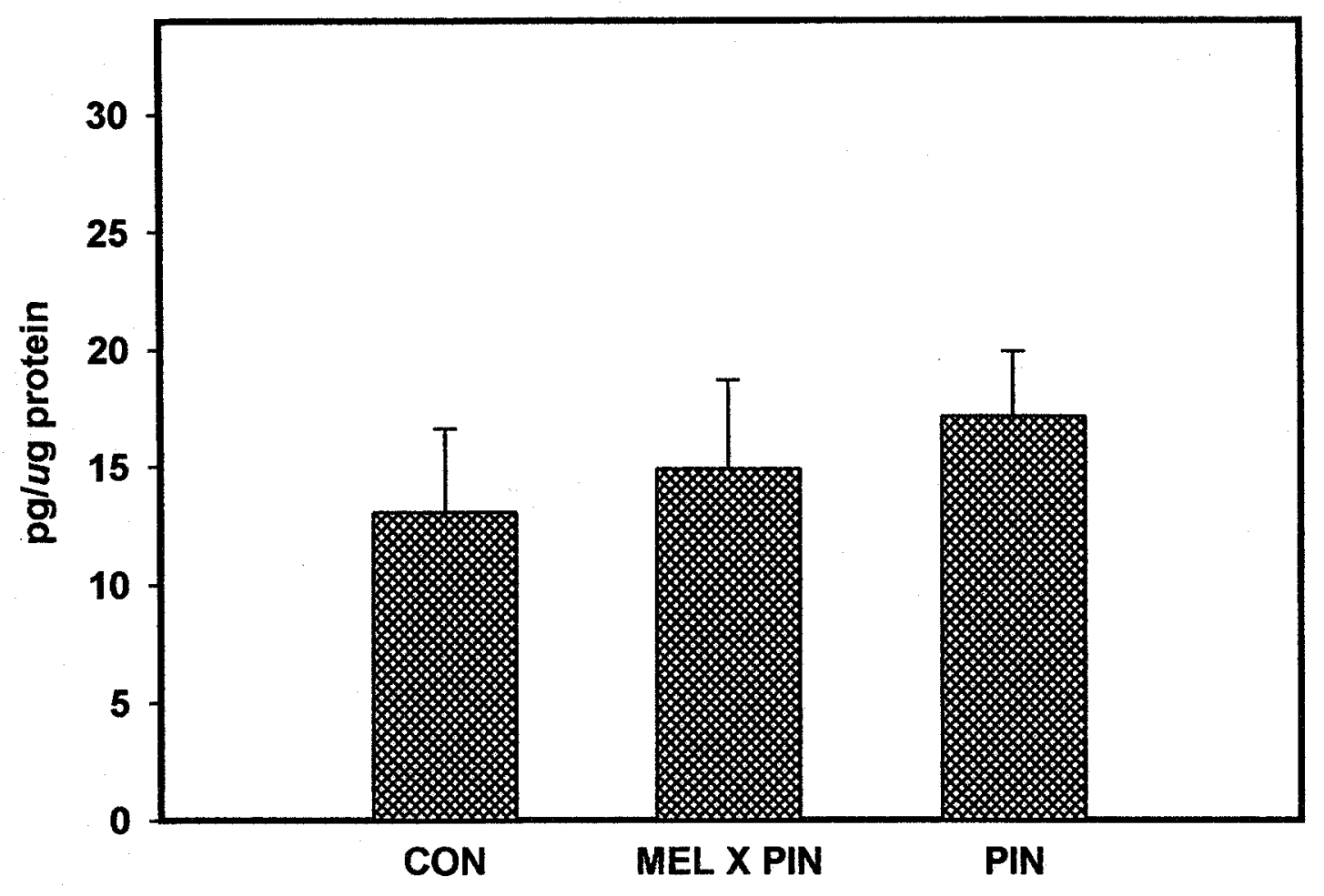


Table 4. Age effects on hippocampal glutathione levels. A significant reduction in hippocampal GSH levels was found in the aged compared to the young animals $(F(1,37)=5.4, p<.03)$. Pinealectomized rats had significantly higher GSH to GSSG ratio compared to the older pinealectomized rats $(F(2,37)=4.79, p<.02)$.

\begin{tabular}{|c|c|c|c|}
\hline Measure & 3-month & 20-month & $\mathbf{p}$ \\
\hline GSH & $107.80 \pm 17.04$ & $61.61 \pm 11.21$ & .030 \\
\hline GSSG & $4.18 \pm .80$ & $4.61 \pm .70$ & .750 \\
\hline GSH/GSSG & $40.25 \pm 8.81$ & $15.03 \pm 1.89$ & .002 \\
\hline
\end{tabular}




\section{Discussion}

The principal results of this project may be summarized as follows.

1. There was a significant difference in mortality rate after 12 months of age among thegroups. The rate $(35 \%)$ was highest for the pinealectomized group and significantly differed from the melatonin supplemented pinealectomized rats $(0 \%)$.

2. When tested at 2 months post surgery on the elevated plus maze, pinealectomized rats spent a greater proportion of time on the central hub compared to the sham rats. No other group differences were observed at this age nor were differences between the three groups found on any of this task's measures at 7 and 11 months post-surgery. At 15 months post-surgery, the melatonin-supplemented pinealectomized rats exhibited increased locomotor activity compared to the sham rats.

Significant age effects were observed for all behavioral measures on the elevated plus maze task. Older rats exhibited fewer entries into the open arms compared to the younger rats. Moreover, older animals exhibited fewer exploratory and locomotor behaviors compared to the younger animals.

3. Following elevated plus maze testing, animals at the 2, 7, and 11-month time-point were tested on a working memory version of the morris water maze task. No significant impairment was observed on the morris water maze following 2,7 , or 11 months of pinealectomy. Age-related deficits in the Morris water maze were observed as 11-month rats performed more poorly than the 2 -month rats.

4. Pinealectomy caused a significant loss of hippocampal CAl pyramidal cells between 2 and 4 months following surgery. Loss of cells in the CA3 was evident at 2 months post- 
pinealectomy. Melatonin replacement in the drinking water prevented these effects of pinealectomy.

5. Biochemical analyses of hippocampal TBARS levels at 3 and 20 months post-surgery, revealed no significant differences between the sham, melatonin supplemented, and pinealectomized groups. At 20 months of age, TBARS levels of the hippocampus were significantly elevated compared to that seen at 3 months of age. Additionally, biochemical analyses of hippocampal reduced glutathione, oxidized glutathione, and ratio of reduced glutathione to oxidized glutathione levels at 3 and 20 months post-surgery, revealed that the pinealectomized rats exhibited a significantly higher ratio of reduced to oxidized glutathione at 3-months compared to the sham and melatonin-supplemented rats. This elevation was not found at the 20 -month time-point. Moreover, reduced glutathione levels were significantly higher in the younger compared to the older animals.

\section{General Health}

An interesting finding in our study was that the death rate was higher in the older pinealectomized group than in the older sham and melatonin- supplemented groups. Specifically, it was found that $35 \%$ of the 20 pinealectomized rats who were designated for analysis at 17 months of age died between 12 and 17 months. It is noteworthy that Reiter et al. (1999) reported that a greater number of their older pinealectomized rats died compared to sham controls. Of the 14 pinealectomized rats that began their study, six died by the time they reached 25 months of age. Reiter and colleagues did not include a group that received melatonin supplementation therefore it is not known whether melatonin would have decreased this mortality rate. 
group that received melatonin supplementation therefore it is not known whether melatonin would have decreased this mortality rate.

Strikingly, it was found here that no melatonin supplemented pinealectomized rats died at any time during the course of this experiment. This zero death rate significantly differed from that (35\%) of the comparable non-supplemented pinealectomized rats. Other research, which has maintained animals until death has shown that exogenously administered melatonin is capable of prolonging the survival of rodents (Pierpaoli \& Regelson, 1994). Pierpaoli \& Regelson (1994) administered melatonin (10 ug/ml) in the drinking water to aging mice (15 months of age) and found that it significantly prolonged survival by 6 months. Melatonin prolonged survival of mice from 23.8 months to 28.1 months. Moreover, treatment with melatonin not only increased survival but also maintained the animals in a more youthful state into advanced age.

\section{Behavioral Findings}

\section{Elevated Plus Maze}

As previously described, the elevated plus maze is often used to assess various behaviors such as anxiety, locomotion, and exploration (Wall \& Messier, 2001). Behavioral indexes of anxiety include the number of open arm entries, ratio of open entries/total entries, percentage of time spent in the open arms and the ratio of protected stretch attend postures made from the protected (closed) arms. These behaviors have typically been used as indices of anxiety because they are found to be increased by anxiolytic drugs and decreased by anxiogenic ones (Dawson \& Tricklebank, 1995). Percentage of time spent in the central hub has been considered a decision and 
approach/avoidance conflict index (Wall \& Messier, 2001). Total number of entries, and the number of entries in closed arms have been considered to be indices of locomotion while the number of vertical stretches in closed arms, and the number of head dips in open arms, have been considered indices of exploration (Wall \& Messier, 2001).

When tested at 2 months post surgery, pinealectomized rats spent a significantly greater proportion of time in the central hub compared to the sham animals. The central hub of the elevated plus maze is a location where rats must decide whether to approach or avoid the unprotected open arms. It has been suggested that spending a significantly greater amount of time in the central hub reflects hesitancy in entering an arm and hence may be an indicator of anxiety (Lee \& Rodgers, 1990). On the other hand, it has also been argued that this measure does not reflect a separate anxiety construct and therefore may not be quite as meaningful as the other measures (Wall \& Messier, 2001).

Thus, the finding that pinealectomized animals spent more time in the central hub than sham animals is difficult to interpret. If this measure were indeed reflective of an increase in anxiety, these animals should have also exhibited significant differences on the more common anxiety indices, such as the number of open entries and proportion of time spent on the open arms, compared to the sham animals. However, pinealectomized animals did not differ on these measures and hence, it cannot be convincingly argued that they were more anxious.

Only one other study has tested the effects of pinealectomy on the elevated plus maze task (Appenrodt \& Schwarzberg, 2000). Unlike the current study, these investigators only measured the percentage of time spent on the open and closed arms as indexes of anxiety, and the number of square entries as an index of locomotor activity. 
Appenrodt \& Schwarzberg (2000) found that pinealectomy in young rats did not affect anxiety-related behavior one-month post-surgery. Consistent with our findings, pinealectomized animals did not differ in the percentage of time spent on the open arms compared to the sham operated controls.

To date, we are the only laboratory to have included an extensive array of measures in order to assess the behavior of pinealectomized rats on the elevated plus maze. The finding of an increased central hub time ratio in the pinealectomized rats was only observed 2 months following surgery and not at any other time-point. Hence, it is possible that this effect was simply an artifact. On the other hand, given the fact that the neuropathological consequences of pinealectomy occurred between 2 and 4 months postsurgery (to be discussed in a later section), and that these changes stabilized after 4 months, it may not be spurious that this behavioral effect was only found at the earliest time-point.

No difference was found between the pinealectomized and melatonin supplemented rats or between the melatonin supplemented and sham rats on this measure. The finding that melatonin supplemented rats did not differ from the sham rats could be because melatonin partially reversed this effect. This can be partly explained by the fact that melatonin has been shown to have anxiolytic properties (Golombek et al., 1996; Golombek et al., 1993; Guardiola et al., 1992; Nava \& Carta; 2001). In a recent study, Nava \& Carter (2001) examined whether melatonin administration ( 4 and $6 \mathrm{mg} / \mathrm{kg}$, i.p.) could reduce anxiety in rats that were administered lipopolysaccharide.

Lipopolysaccharide is a bacterium that induces fever, reduction in locomotor activity, and increased anxiety in rodents. These investigators found that the acute administration of 
melatonin $1,2,3$, and 4 hours prior to elevated plus maze testing significantly reduced the anxiety behaviors on this maze. Melatonin treated rats made significantly more entries into the open arms and spent more time in these arms compared to the non-treated lipopolysaccharide rats. In their study, Nava \& Carter (2001) administered melatonin to pineal intact animals to study its anxiolytic effects. In fact, the majority of studies that have examined melatonin's anxiolytic properties have administered melatonin to young pineal-intact animals whose plasma levels of this hormone are already high (Arushanian et al. 2003; Golombek et al. 1993; Kopp et al. 1999). Moreover, large concentrations of melatonin are often used in these studies (Nava \& Carter, 2001). Because we administered melatonin to pinealectomized rats, their melatonin levels would have been lower than those seen in these other studies. This may partly explain why the melatonin supplemented rats did not significantly differ from the pinealectomized rats on central hub time.

Additional research will need to be done in order to definitively conclude that early pinealectomy increases decision-making and approach/avoidance conflict on the elevated plus maze.

Elevated plus maze testing at 15 -months post-surgery revealed that the melatonin -supplemented rats exhibited significant greater locomotor activity (total open and closed arm entries) the sham rats. Moreover, narrowly missing significance was the finding that these melatonin- supplemented rats entered more closed arms compared to the other two groups.

This finding is consistent with research by Appenrodt \& Schwartzberg (2000) who reported that administration of melatonin $(250 \mathrm{ug}$, s.c. $)$ to pinealectomized rats for 3 
days prior to testing, significantly increased locomotor activity compared to sham operated controls. It is well known that aging in rodents is associated with varying degrees of behavioral impairments (Cantuti-Castelvetri et al., 2000) on behavioral tasks such as the elevated plus maze (Laviola et al., 2003), Morris water maze (Magnussun et al., 2003), and plus-maze and passive avoidance (Raghavendra \& Kulkarni, 2001). According to the free radical theory of aging, both age-related neuronal and behavioral changes are thought to occur as a result of an accumulation of free radicals across the lifespan and the oxidative stress they generate (Reiter, 1995). Several studies have investigated whether antioxidant supplementation is capable of reversing various agerelated behavioral deficits in rodents (Cantuti-Castelvetri et al., 2000; Goyarzu et al., 2004; Joseph et al., 1999). For instance, Joseph and colleagues (1999) found that aged rats given dietary antoxidant supplementation demonstrated a significant reversal of agerelated cognitive and motor behavioral deficits on various tasks such as the rod walking and accelerod tasks as well as Morris water maze task. Chronic melatonin treatment in aged mice has also been found to reverse age-induced retention deficits in both a passiveavoidance and elevated plus maze task (Raghavendra \& Kulkarni, 2001). In support of the evidence demonstrating beneficial effects of antioxidants in reversing age-induced behavioral changes, the present study found that chronic administration of melatonin significantly increased locomotor activity among the older rats on the elevated plus maze task.

A number of studies have found that aging markedly affects rats' behavior on the elevated plus maze (Laviola et al., 2003; Macri et al., 2002). Adolescent rats (post-natal period ranging from 21 to 60 days) show less anxiety-related behavior and are more 
likely to engage in risk taking behavior compared to both juvenile and adult rats (Laviola et al., 2003; Macri et al., 2002). Older rats are more inhibited and are less likely to explore the maze. They also show a marked aversion for the unprotected areas of the maze and tend to spend a proportionately greater time in the protected areas of the maze. Consistent with this literature, age-related differences were found in the current study for all of the behavioral measures. Older rats demonstrated more anxiety-related behaviors and decreased levels of locomotion and exploration compared to the 2 -month postsurgery animals. They entered fewer arms and spent significantly more time in the protected rather than the unprotected arms. As well, the number of protected stretch attend postures, an indicator of risk assessment (Wall \& Messier, 2001) was decreased in the older animals.

\section{Morris water maze}

Testing on the working memory version of the Morris water maze, revealed no significant deficits in working memory following 2,7 , or 11 months of pinealectomy. Pinealectomized animals similar to both the sham and melatonin supplemented animals, were able to learn this task as measured by both latency and the distance swum to locate the hidden platform. This is consistent with our previous finding that pinealectomy did not affect working memory as assessed by both a tactile and a standard radial arm maze task (De Butte et al., 2002). It is also consistent with other findings that they are not different on passive and active avoidance tasks (Appenrodt \& Schwartzberg, 1999; Juszczak et al., 1996), and social memory tasks (Appenrodt et al., 2002). 
The testing of rats at various ages revealed significant age-related deficits in working memory. Specifically, the 11-month post-surgery rats exhibited longer latencies to find the platform on the first and second testing day compared to the 2-month rats. This is consistent with numerous studies that have found age-related deficits in the acquisition of the Morris water maze (Gallagher \& Pelleymounter, 1988; Shukitt-Hale et al., 1998) and radial arm maze (Kobayashi et al., 1988; Wallace et al., 1980) tasks. For example, Magnusson and colleagues (2003) found a significant decline in working memory performance at 10 months of age. Although rats are not considered aged at 10 months, it has been suggested that working memory is affected at a much earlier age compared to other memory systems such as reference memory (Magnusson et al., 2003).

\section{Hippocampal Pathology}

\section{CAI and CA3 Cell Number}

Previous work in our laboratory demonstrated that 16-months of pinealectomy caused a significant loss of neurons in the CA1 and CA4 sectors of the hippocampus (De Butte et al., 2002). Because these animals were examined at 16 months of age, the temporal evolution of this neuronal loss was not known. Therefore, a major aim of the current study was to examine the brains of rats at 2-month intervals after shampinealectomy and pinealectomy surgery in order to determine when these neurons degenerate. In addition to the $\mathrm{CA} 1$, we also examined the $\mathrm{CA} 3$ region for the first time. It was also an intention of the present study to determine if melatonin supplementation would reverse the effects of pinealeactomy. If the neuropathology associated with 
pinealectomy was indeed due to a loss of this indole, then the effects should have been reversed in pinealectomized rats treated with melatonin.

Consistent with our previous report (De Butte et al., 2002), a significant loss of hippocampal pyramidal cells was observed in both the CA1 and CA3 sectors. Moreover, supplementation with melatonin in the drinking water completely reversed this loss of CA1 and CA3 pyramidal cells. Two months post-surgery, no significant differences in CA1 cell number were found between the groups. The CA1 cell loss occurred between $2-$ 4 months after pinealectomy. At 4 months, pinealectomized rats showed a $25 \%$ reduction in cell number compared to the sham rats. Furthermore, at least a $15 \%$ reduction in cell number was found at every interval thereafter.

Melatonin replacement partially protected against this cell loss at 4 months postsurgery and completely protected against it at every time-point thereafter. Remarkably, not only did melatonin supplementation result in the complete reversal of the effects caused by pinealectomy, but it also caused a significant $10 \%$ increase of neurons in the CA1 region at 8-months post-surgery.

Similar to the CAl region, pinealectomy also caused a significant reduction of CA3 cells and this too was prevented by melatonin supplementation. Unlike the CA1 region, an $18 \%$ loss of cells was observed 2 -months after pinealectomy. Pinealectomized rats exhibited a significant cell loss of at least $19 \%$ compared to the sham rats at every time-point except at the 17-month time-point. Similar to the CA1, melatonin supplementation afforded partial protection at the first time-point but then afforded full protection thereafter. No significant increase in CA3 cell number was found at any timepoint in the melatonin supplemented rats. 
The most obvious interpretation of this data is that because melatonin seemingly exerts potent antioxidant and neuroprotective properties (Reiter, 1991), its reduction after pinealectomy caused the death of hippocampal pyramidal cells. That melatonin supplementation afforded full protection against this cell loss confirms that the reduction of this hormone played a significant role in the effect of pinealectomy. These findings provide further support that melatonin has remarkable neurotrophic properties and is consistent with a growing number of studies that have demonstrated its ability to protect against various central nervous system insults such as ischemia/reperfusion injury (Lee et al., 2004; Manev et al., 1996; Torii et al., 2004; Pei \& Cheung, 2004), excitotoxic injury (Manev et al., 1996; Melchiorri et al., 1995) and in models of Alzheimer's (Matsubara et al., 2003; Pappolla et al., 2000) and Parkinson's (Antolin et al., 2002; Jin et al., 1998; Kim et al., 1998; Mayo et al., 1999; Thomas \& Mohanakumar, 2004) disease.

\section{Fluoro-Jade B Staining}

Fluoro-Jade B staining in the hippocampus revealed that the pinealectomized rats did not differ in the number of Fluoro-Jade B positive cells compared to the sham animals at all of the time-points examined. Stereological counts of the hippocampal CA1 and $\mathrm{CA} 3$ regions indicated that the majority of the cell death occurred prior to the 2month time-point for the CA3, and between the 2 and 4-month time-point for the CA1. Hence, it is not surprising that the number of Fluoro-Jade positive cells did not differ between the pinealectomized and sham rats at the 2 and 4-month time-points because most of the cell loss would have already occurred. Furthermore, the finding of no differences in Fluoro-Jade B staining between the pinealectomized and sham animals at 
later time-points is consistent with the finding that the cell loss stabilized after 2 months of pinealectomy in the $\mathrm{CA} 3$ and 4 months of pinealectomy in the $\mathrm{CA} 1$ regions.

A compilation of all the Fluoro-Jade B data from the different regions of the hippocampus, revealed a significant difference between the groups at the 4-month timepoint. Melatonin supplemented rats exhibited fewer Fluoro-Jade B positive cells at this time-point compared to the pinealectomized rats. This is an interesting finding because at the 4-month time-point, melatonin supplementation only partially protected against the CA1 cell loss. At 6 months, CAl cell number did not differ between the melatonin supplemented and sham rats suggesting an increase in cell number that occurred between the 4 and 6-month intervals. The hippocampus undergoes a significant period of naturally occurring cell death accompanied by neurogenesis throughout development and adulthood (Dayer et al. 2003). The present results suggest that while the pinealectomized rats were undergoing hippocampal cell death at 4 months of age, cell death was inhibited in the melatonin- supplemented rats. This phenomenon may offer an explanation as to why the melatonin supplemented rats exhibit partial cell loss at 4-months of age, but cell number's comparable to the sham rats at 6 months of age. If cell death was inhibited but neurogenesis was still occurring, this may explain the significant increase in CA1 cell number at the next time-point ( 8 months). To address this issue, future studies will have to determine whether there is increased neurogenesis in the hippocampus at these timepoints.

In this regard, it should be noted that recent research documents neurogenesis in the CA1 in rats and gerbils following transient global ischemia (Kokaia \& Lindvall, 2003; Nakatomi et al., 2002; Schmidt \& Reymann, 2002). For example, Schmidt \& 
Reymann (2002) found marked CA1 cell loss 7 days following global cerebral ischemia in gerbils. Significant neurogenesis was then observed 4 weeks later in the CA1 pyramidal cell layer. Similarly, Nadatomi and colleagues (2002) found a gradual loss of CA1 neurons 2-4 days after forebrain ischemia in rats. These investigators then found that hippocampal CA1 pyramidal neurons were regenerated 1-month following ischemia. The current experiment hints that CAl neurogenesis is promoted by melatonin-however this observation was made only for pinealectomized rats who speculatively, may share some features with ischemic rats. Hence, it would also be worthwhile to investigate if melatonin supplementation affects CA1 neurogenesis in otherwise intact rats.

\section{TBARS and Glutathione Levels}

Results obtained from this study indicate that the chronic lowering of melatonin results in a significant loss of neurons in the hippocampus. Ample studies have demonstrated that melatonin has potent antioxidant and free radical scavenging properties (Reiter, 1998). Therefore it is plausible that pinealectomy, which lowers melatonin, may actually result in an increase in free radical activity eventually causing free radical mediated neuronal loss. To address this issue, the present study measured tissue levels of lipid peroxidation products 3 and 20 months post-surgery. An increase in the levels of lipid peroxidation products such as malondialdehyde, is commonly used as an indicator of increased free radical production (Halliwell \& Gutteridge 1989).

In addition to lipid peroxidation products, tissue levels of glutathione (both reduced and oxidized forms) were also measured in these animals. Glutathione is an important component of the antioxidant defense system and is essential in preventing free 
radical mediated cellular damage. Glutathione acts as a reductant by the oxidation of its thiol group. The oxidized form of glutathione is produced as a result of glutathione oxidation. Tissue levels of reduced and oxidized glutathione are often used as indices of oxidative stress. Generally, cellular levels of reduced glutathione are high as is the ratio of reduced glutathione to oxidized glutathione (Hara et al., 1996). Decreases in reduced glutathione levels as well as in the ratio of reduced glutathione to oxidized glutathione have been used as further indicators of oxidative stress. As well, a significant increase in the ratio of reduced glutathione to oxidized glutathione, is reflective of an up-regulation of this system and is also an indicator of an increase in free radical activity.

In the current study, pinealectomized rats from both age groups did not show an elevation of lipid peroxidation products compared to the sham and melatonin supplemented rats. This is contrary to previous reports that have found a significant increase in lipid peroxidation in these animals. For instance, Baydas and colleagues (2002) examined levels of lipid peroxidation products in the brains of pinealectomized rats and found that malondialdehyde brain levels were significantly increased following one-month of pinealectomy. In an earlier study, these investigators suppressed melatonin synthesis by exposing rats to constant light, a procedure called functional pinealectomy. This technique inhibits the normal nocturnal surge of melatonin but does not severely reduce diurnal levels like surgical pinealectomy. It was found that two weeks of constant light exposure significantly increased lipid peroxidation (as measured by malondialdehyde levels) in rat cerebral hemisphere (Baydas et al., 2001).

Although the current study did not find an increase of lipid peroxidation at the 3 month time-point, a significant increase in the ratio of reduced glutathione to oxidized 
glutathione was observed in the hippocampus of pinealectomized rats. This is suggestive of an up-regulation of the glutathione system at this time-point. Based on previous reports, it is quite plausible that pinealectomized animals exhibit higher lipid peroxidation levels shortly following pinealectomy but that a compensatory change in the glutathione system is required to effectively counteract this increase in lipid peroxidation. Hence, the finding in the present study of an increase in the ratio of reduced glutathione to oxidized glutathione in the hippocampus of pinealectomized rats may be indicative of such a compensatory change.

Taken together, it could be argued that the initial cascade of cell loss observed in the present study is a direct result of an increase in free radical activity shortly following pinealectomy but that the cessation of cell loss is due to the up-regulation of the glutathione defense system.

\section{Effect of Age on Hippocampal Measures}

In the present study, stereological counting revealed a significant age-related loss of neurons in the $\mathrm{CA} 3$ region of the hippocampus in the sham and pinealectomized rats. Specifically, a $25 \%$ loss of CA3 neurons was observed in the 17-month sham rats compared to the 2-month sham rats. Similarly, older pinealectomized rats exhibited a $20 \%$ loss of neurons compared to the 4-month old pinealectomized rats. Melatonin supplemented rats did not show this age-related neuronal loss. Moreover, agerelated cell loss in the CAl region was observed among the pinealectomized rats.

Unlike the present research, several other stereological studies have found that neuron number in the CAl region is preserved in aged rats (Rapp \& Gallagher, 1996; 
Rasmussen et al. 1996). Similarly, these previous reports did not find any cell loss in the CA3 region of the hippocampus. Rapp \& Gallagher (1996) utilized the optical fractionator method to estimate total cell number in the CA1 and CA3 sectors of the hippocampus. These investigators surveyed the pyramidal cell fields at evenly spaced $x-y$ intervals of $220 u \mathrm{~m}$ by $220 u \mathrm{~m}$. Using this sampling parameter, these investigators reported that they counted approximately 191 cells in the CA3 region per hippocampus.

The current study used a more stringent interval of $90 u \mathrm{~m}$ by $90 u \mathrm{~m}$ and counted $>700$ cells per $\mathrm{CA} 1$ and CA3 region. Stereological techniques allow for the detection of subtle differences in cell number but it may be more difficult to statistically detect these differences if not enough cells have been counted. Because we counted a greater number of cells per brain, this may account for why we were able to detect age-related differences in $\mathrm{CA} 1$ and $\mathrm{CA} 3$ cell number.

In the present study, TBARS levels in the hippocampus were found to be significantly higher in the 20 -month rats compared to the 3 -month rats. Several studies have found that the oxidative status of aged animals is higher as compared with young animals (Akbulut et al., 1998; Reiter et al., 1999; Zhang et al., 1993). Akbulut and colleagues (1998) found significantly elevated malondialdehyde levels in plasma, lung, and liver of aged rats compared to young rats. Similarly, Reiter et al. (1999) reported elevated levels of lipid peroxidation products in the lung, kidney, and skin of 25-month rats compared to 2 -month rats.

Reiter and colleagues (1999) further examined whether pinealectomy would exacerbate this age-related increase in lipid peroxidation levels in several peripheral tissues (lung, kidney, pancreas, testes) as well as in the brain (hippocampus and brain 
stem) of 25-month old rats that had been pinealectomized at two months of age. These investigators found a significant increase in lipid peroxidation products in the hippocampus and brain stem of pinealectomized aged rats compared to aged-matched controls. An increase in lipid peroxidation products was also found in several peripheral tissues such as the lung, pancreas, and muscle of these rats. In contrast to these findings, the present study did not find higher TBARS levels in the older pinealectomized rats compared to the older sham and melatonin- supplemented rats. One possible reason for this discrepancy is that our older pinealectomized animals were much younger than those used in the Reiter et al. study. The finding that the ratio of reduced glutathione to oxidized glutathione was not elevated in the older pinealectomized rats suggests that unlike the younger pinealectomized rats, their glutathione system was not up-regulated to compensate for the loss of melatonin. It is likely that over time, these animals may exhibit significantly higher tissue levels of lipid peroxidation as demonstrated in the Reiter et al. (1999) study.

Generally, a finding of increased tissue levels of lipid peroxidation is also associated with a depletion of reduced glutathione levels (Akbulut et al., 1999; Hara et al., 1996). This is not surprising because glutathione is a key component of the antioxidant defense system and its activity is usually increased in response to an increase in free radical activity. With an increase in free radical burden, reduced glutathione levels are depleted.

Similar to lipid peroxidation levels, glutathione levels have also been found to show age-related changes. For example, Akbulut et al. (1999) found not only an agerelated increase in malondialdehyde in the lung, but also a significant decrease in reduced 
glutathione levels. Similar to this study, we also found a significant decrease in reduced glutathione in the hippocampus of the older rats compared to younger rats. This is consistent with the free radical theory of aging, which postulates that free radical damage accumulates with aging and places an increased demand on the antioxidant defense system.

\section{Summary and Conclusions}

Melatonin not only regulates biological rhythms, but accumulating evidence suggests that it also functions as an antioxidant and free radical scavenger (Reiter, 1998; Reiter et al., 2000; Tan et al., 1993). As such, it appears to protect cells from a variety of insults, thus prolonging their life and the general viability of organisms. The results from the present study add further support to this. It was discovered here that the reduction of endogenous melatonin by pinealectomy increased mortality rate and caused a significant loss of hippocampal CA1 and CA3 pyramidal cells 2-4 months post-surgery. Melatonin supplementation in the drinking water of pinealectomized rats not only reversed these effects, but also seemed to promote the genesis of new CA1 cells. Although pinealectomized rats did not exhibit an elevation of lipid peroxidation levels in the hippocampus at 3-months of age, they did exhibit an up-regulation of the glutathione system suggesting they were experiencing elevations in free radical activity at that timepoint. That free radical activity was increased in the hippocampus at 3-months following pinealectomy is in concordance with the finding of hippocampal cell death at the 4-month time-point. 
To date, researchers including ourselves have studied the effects of pinealectomy in rats that were pinealectomized at a very young age ( $<2$ months). Future research should determine if the effects pinealectomy that were observed here are peculiar to the age when the surgery was performed. Pinealectomy was performed here prior to puberty. Would similar effects be observed if the surgery was performed post puberty? Would the effects be even more profound in more aged animals, when endogenous antioxidant systems are in decline?

Furthermore, it will be important to further explore the suggestion here that melatonin caused the regeneration of hippocampal CA1 cells in pinealectomized animals. This could be accomplished by the labeling of newly formed CA1 neurons with a specific marker such as 5-bromo-2-deoxyuridine (BRDU), which selectively labels proliferative cells in vivo. In addition to examining the effects of melatonin on CAl cell birth in pinealectomized animals, it should also be determined if it causes this in intact animals.

As reviewed earlier, evidence suggests a reduction of melatonin levels in Alzheimer's patients (Zhou et al., 2003). Since CA1 pyramidal cell loss is a hallmark feature of early stage Alzheimer's (Selkoe, 1996). The results here as well as our earlier report (De Butte et al., 2002) indicate that the reduction of endogenous melatonin promotes cell loss, thus reinforcing the possibility that reduced melatonin may be pathogenic to Alzheimer's.

Although pinealectomy resulted in the loss of cells in the hippocampus, it did not result in impairments in working memory. One reason for this finding is that the cell loss in both the CA1 and CA3 was a modest one $(<27 \%)$ and hence, was simply not sufficient enough to disrupt memory processes. This finding was in accordance with several 
studies that have demonstrated that pinealectomized rats are not impaired on several behavioral paradigms (Appenrodt \& Schwartzberg, 1999; Appenrodt et al., 2002; Juszczak et al., 1996). Moreover, pinealectomy did not result in an increase in anxietyrelated behaviors on the elevated plus maze. Although pinealectomy did not cause an increase in anxiety, it would be of interest to assess whether these animals are more susceptible to stress. In intact animals, melatonin has demonstrable anxiolytic properties (Golombek et al., 1996). Pinealectomy which significantly lowers this hormone, could result in an increased susceptibility to stress- induced neuropathology and behavioral impairment. Psychological stressors cause oxidative stress and inflammation (Behl, 1998; Esch et al., 2002), processes that are counteracted by melatonin.

Substantial evidence supports the idea that aging in the brain may be related to damage caused by oxygen reactive species (Halliwell, 1992; Harman, 1992; Harman, 1994). In the present study, rodents were found to exhibit an age-related working memory impairment as well as cell loss in the CA3 and CA1. Moreover, TBARS levels were significantly increased in the older rodents and reduced glutathione levels were depleted, supporting other evidence that the generation of free radicals increases with normal aging and that antioxidative enzyme activities may diminish in effectiveness (Harman, 1981; Johnson et al., 1999). This increase in free radical activity without a commensurate increase in antioxidantive enzymes, is believed to damage cells and cause the pathophysiological changes with aging. In contrast to Reiter et al. (1999), the present study did not find that pinealectomy exacerbated age-related effects. However, our animals were much younger than those of Reiter et al and hence, it would be of interest to examine whether pinealectomized rats would show further neuronal effects when they are 
aged ( $\sim 25$ months). Moreover, it would also be informative to examine the activity of other antioxidant enzymes such as glutathione and catalase at 25 months of age and determine whether the decreases typically seen in aged rats are exacerbated in pinealectomized rats.

In conclusion, the results of the present study indicate that melatonin is a neuroprotectant in the brain. Chronic oral melatonin supplementation did not have any toxic effects suggesting that it can be safely ingested over extended periods of time. Melatonin may offer considerable clinical benefit as a protective agent against neurodegenerative conditions such as Alzheimer's disease that are at least partly attributable to oxidative damage. 


\section{REFERENCES}

Acuna-Castroviejo, D., Coto-Momtes, A., Gaia Monti, M., Oritz, G.G., \& Reiter, R.J. (1997). Melatonin is protective against MPTP-induced striatal and hippocampal lesions. Life Sciences, 60, 23-29.

Akbulut, K.G., Gonul, B., \& Akbulut, H. (1999). Differential effects of pharmacological doses of melatonin on malondialdehyde and glutathione levels in young and old rats. Gerontology,45, 67-71.

Alam, Z.I., Jenner, A., Daniel, S.E., Lees, A.J., Cairns, N., Marsden, C.D., Jenner, P., \& Halliwell, B. (1997). Oxidative DNA damage in the parkinsonian brain: An apparent selective increase in 8-hydroxyguanine levels in substantia nigra. Journal of Neurochemistry, 69, 1196-1203.

Andrade, M.M., Tome, M.F., Santiago, E.S., Lucia-Santos, A., Andrade, T.G. (2003). Longitudinal study of daily variation of rats' behavior in the elevated plus maze. Physiology and Behavior, 78, 125-133.

Antolin, I., Mayo, J.C., Sainz, R.M., de los Angeles del Brio, M., Herrera, F., Martin, V., \& Rodriguez, C. (2002). Protective effect of melatonin in a chronic experimental model of Parkinson's disease. Brain Research, 943, 163-173.

Appenrodt, E., Juszczak, M., \& Schwarzberg, H. (2002). Septal vasopressin induced preservation of social recognition in rats was abolished by pinealectomy. Behavioural Brain Research, 134, 67-73.

Appenrodt, E., \& Schwarzberg, H. (2000). Central vasopressin administration failed to influence anxiety behavior after pinealectomy in rats. Physiology and Behavior, $68,735-739$.

Appenrodt, E., \& Schwarzberg, H. (2003). Pinealectomy blocks modulation of active avoidance by central vasopressin application in rats. Peptides, 24, 129-136.

Armstrong, S.M. (1989). Melatonin and circadian control in mammals. Experientia, 45, 932-945.

Arushanian, E.B., Baturina, M.V., Beier, E.V., \& Kuz'mina E.M. (2003). Effects of anxiolytic agents on some immunologic parameters of the stressed rats. $E k s p$ Klin Farmakol., 6, 45-47.

Barlow-Walden, L.R., Reiter, R.J., Abe, M., Pablos, M., Menendez-Pelaez, A., Chen, L.D., \& Poeggeler, B. (1995). Melatonin stimulates brain glutathione peroxidase activity. Neurochemistry International, 26, 497-502. 
Baydas G., Gursu, M.F., Yilmaz, S., Canpolat, S., Yasar, A., Cikim, G., \& Canatan, H. (2002). Daily rhythm of glutathione peroxidase activity, lipid peroxidation and glutathione levels in tissues of pinealectomized rats. Neuroscience Letters, 323, 195-198.

Behl, C., Davis., J.B., Lesley, R., Schubett, D. (1994b). Hydrogen peroxide mediates amyloid B protein toxicity. Cell, 77, 817-827.

Brotto, L.A., Gorzalka, B.B., \& LaMarre, A. (2001). Melatonin protects against the effects of chronic stress on sexual behavior in male rats. Neuroreport, 12, 34653469.

Brzczinski, A. (1997). Melatonin in humans. The New England Journal of Medicine, 336, 186-195.

Cagnacci, A. (1996). Melatonin in relation to physiology in adult humans. Journal of Pineal Research, 21, 200-213.

Carneiro, R.C.G., \& Reiter, R.J. (1998). Melatonin protects against lipid peroxidation induced by aminolevulinic acid in rat cerebellum, cortex, and hippocampus. Neuroscience, 82, 293-299.

Cantuti-Castelvetri, I., Shukitt-Hale, B., \& Joseph, J.A. (2000). Neurobehavioral aspects of antioxidants in aging. International Journal of Developmental Neuroscience, $18,367-381$.

Cavallo, A. (1993). The pineal gland in human beings: Relevance to pediatrics. The Journal of Pediatrics, 123, 843-851.

Cohen, G. (1984). Oxy-radical toxicity in catecholamine neurons. Neurotoxicology, 1 , 77-82.

Cotman, C.W. (1998). Apoptosis decision cascades and neuronal degeneration in Alzheimer's Disease. Neurobiology of Aging, 19, S29-S32.

Cuzzocrea, S., Costantino, G., Otto, E., Mazzon, E., Fulia, F., Serraino, I., Cordaro, S., Barberi, I., De Sant, Y., Caputi, A.P. (2000). Protective effects of melatonin in ischemic brain injury. Journal of Pineal Research, 29, 217-227.

Dawson, G.R., \& Tricklebank, M.D. (1995). Use of the elevated plus maze in the search for novel anxiolytic agents. Trends in Pharmacological Science, 16, 33-36.

Dayer, A.G., Ford, A.A., Cleaver, K.M., Yassaee, M., \& Cameron, H.A. (2003). Shorttermand long-term survival of new neurons in the rat dendate gyrus. Journal of Comparative Neurology, 4, 563-572. 
De Butte, M., Fortin, T., \& Pappas, B.A. (2002). Pinealectomy: behavioral and neuropathological consequences in a chronic cerebral hypoperfusion model. Neurobiology of Aging, 23, 309-317.

Dykens, J.A., Stern, A., \&. Trenkner, E. (1987). Mechanism of kainate toxicity to cerebellar neurons in vitro is analogous to reperfusion tissue injury. Journal of Neurochemistry, 49, 1222-1228.

Ebadi, M., Gouitrapong, P., Phansuuvan-Pujito, P., Nelson, F., \& Reiter, R.J. (1998). Pineal opioid receptors and analgesic action of melatonin. Journal of Pineal Research, 24, 193-200.

Ebling, F.J.P., \& Foster, D.L. (1989). Pineal melatonin rhythms and the timing of puberty in mammals. Experentia, 45, 946-954.

Esch, T., Stefano, G.B., Friccione, G.L., \& Benson, H. (2002). The role of stress in neurodegenerative diseases and mental disorders. Neuroendocrinology Letters, $23,199-208$.

Fridovich, I. (1983). Superoxide radical: An endogenous toxicant. Annual Review of Pharmacology and Toxicology, 23, 239-257.

Gallagher, M., \& Pelleymounter, M.A. (1988). Spatial learning deficits in old rats: a model for Memory decline in the aged. Neurobiology of Aging, 9, 549-556.

Giusti, P., Lipartiti, M., Franceschini, D., Schioro, N., Floreani, M., \& Manev, H. (1996). Neuroprotection by melatonin from kainate-induced excitotoxicity in rats. FASEB, 10, 891-896.

Golombek, D.A., Escolar, E., Burin, L., Brito Sanchez, M.G., \& Cardinali, D.P. (1991). Time-dependent melatonin analgesia in mice: Inhibition by opiate or benzodiazepine antagonism. European Journal of Pharmacology, 194, 25-30.

Golombek, D.A., Martini, M., \& Cardinali, D.P. (1993). Melatonin as an anxiolytic in rats: Time-dependency and interaction with the central GABAergic system. European Journal of Pharmacology, 237, 231-236.

Golombek, D.A., Pevet, P., \& Cardinali, D.P. (1996). Melatonin effects on behavior: Possible Mediation by the central GABAergic system. Neuroscience and Biobehavioral Reviews, 20, 403-412.

Goyarzu, P., Malin, D.H., Lau, F.C., Taglialatela, G., Moon, W.D., Jennings, R., Moy, D., Lippold, S., Shukitt-Hale, B., \& Joseph, J.A. (2004). Blueberrry supplemented diet:effects on object recognition memory and nuclear factor-kappa $B$ levels in aged rats. Nutritional Neuroscience, 7, 75-83. 
Guardiola, L.B., Lenegre, A., \& Porsolt, R.D. (1992). Combined effects of diazepam and melatonin in two tests for anxiolytic activity in the mouse. Pharmacology Biochemistryand Behavior, 41, 405.

Haimov, I., Lowie, P., Laudon, M., Herer, P., Vigder, C., Zzisapel, N. (1995). Melatonin replacement therapy of elderly insomniacs. Sleep, 18, 598-603.

Halliwell, B. (1992). Reactive oxygen species and the central nervous system. Journal of Neurochemistry, 59, 1609-1623.

Halliwell, B., \& Gutteridge, D.M.C. (1984). Oxygen toxicity oxygen radical, transition metals and disease. Biochemistry Journal, 219, 1-14.

Hara, M., Abe, M., Suzuki, T., \& Reiter, R.J. (1996). Tissue changes in glutathione metabolism and lipid peroxidation induced by swimming are partially prevented by melatonin. Pharmacology and Toxicology, 78, 308-312.

Harman, D. (1981). The aging process. Proceedings of the National Academy of Sciences USA, 78, 7124-7128.

Harman, D. (1994). Free-radical theory of aging. Increasing the functional life span. Annals of the New York Academy of Sciences, 717, 1-15.

Jin, B.K., Shin, D.Y., Jeong, M.Y., Gwag, M.R., Baik, H.W., Yoon, K.S., Cho, Y.H., Joo, W.S., Kim, Y.S., Baik, H.H. (1998). Melatonin protects nigral dopaminergic neurons from 1-methyl-4-phenylpyridinium (MPP ${ }^{+}$) neurotoxicity in rats. Neuroscience Letters, 245, 61-64.

Joo, J.Y., Uz, T., \& Manev, H. (1998). Opposite effects of pinealectomy and melatonin administration on brain damage following cerebral focal ischemia in rat. RestorativeNeurology and Neuroscience, 13, 185-191.

Joseph, J.A., Shukitt-Hale, B., Denisova, N.A., Bielinska, D., Martin, A., McEwen, J.J., \& Bickford, P.C. (1999). Reversals of age-related declines in neuronal signal transduction, cognitive, and motor behavioral deficits with blueberry, spinach, or strawberry dietary supplementation. The Journal of Neuroscience, 19, 8114-8121.

Juszczak, M., Drobnik, J., Guzek, J.W., \& Schwarzberg, H. (1996). Effect of pinealectomy and melatonin on vasopressin-potentiated passive avoidance in rats. Journal of Physiology and Pharmacology, 47, 621-627.

Karbownik, M., Reiter, R.J., Burkhardt, S., Gitto, E., Tan, D.X., \& Lewinski, A. (2001). Melatonin attenuates estradiol-induced oxidative damage to DNA: Relevance for cancer prevention. Experimental Biology and Medicine, 226, 707-712. 
Kato, K., Murai., I., Asai, S., Matsuno, Y., Komuro, S., Kaneda, N., Iwasaki, A., Ishikawa, K., Nakagawa, S., Arakawa, Y., \& Kuwayama, H. (1998). Protective role of melatonin and the pineal gland in modulating water immersion restraint stress ulcer in rats. Journal of Clinical Endocrinology, 27, S110-S115.

Kehrer, J.P. (1993). Free radicals as mediators of tissue injury and disease. Critical Reviews in Toxicology, 23, 21-48.

Kim, Y.S., Joo, W.S., Jin, B.K., Cho, Y.H., Baik, H.H., \& Park, C.W. (1998). Melatonin protects 6-OHDA-induced neuronal death of nigrostriatal dopaminergic system. Neuroreport, 9, 2387-2390.

Kobayashi, S., Kametani, H., Ugawa, Y., \& Osanai, M. (1988). Age difference of response strategy in radial maze performance of Fischer-344 rats. Physiology and Behavior, 42, 277-280.

Kokaia, Z., \& Lindvall, O. (2003). Neurogenesis after ischaemic brain insults. Current OpinionIn Neurobiology, 13, 127-132.

Kopp, C., Vogel, E., Rettori, M.C., Delagrange, P., \& Misslin, R. (1999). The effects of melatonin on the behavioural disturbances induced by chronic mild stress in $\mathrm{C}_{3} \mathrm{H} / \mathrm{He}$ mice. Behovioural Pharmacology, 10, 73-83.

Lakin, M.L., Miller, C.H., Stott, M.L., Winters, W.D. (1981). Involvement of the pineal gland and melatonin in murine analgesia. Life Science 29, 2543-2551.

Laviola, G., Macri, S., Morley-Fletcher, S., Adriani, W. (2003). Risk-taking behavior in adolescent mice: psychobiological determinants and early epigenetic influence. Neuroscience and Biobehavioral Reviews, 27, 19-31.

Lee, C., \& Rodgers, J.R. (1990). Antinociceptive effects of elevated plus-maze exposure: Influence of opiate receptor manipulations. Psychopharmacology, 102, 507-513.

Lee, E., Wu, T., Lee, M., Chen, T., Tsai, Y., Chuang, J., \& Chang, G. (2004). Delayed treatment with melatonin enhances electrophysiological recovery following transient focal cerebral ischemia in rats. Journal of Pineal Research, 36, 33-42.

Lin, P., \& Bilkey, D.K. (2001). The effect of excitotoxic lesions centered on the hippocampus or perirhinal cortex in object recognition and spatial memory tasks. Behavioral Neuroscience, 1, 94-111.

Lohr, J.B. (1991). Oxygen, radicals and neuropsychiatric illness. Archives of General Psychiatry, 48, 1097-1106. 
Liu, R.Y., Zhou, J.N., Van Heerikhuize, J., Hofman, M.A., \& Swaab, D.F. (1999).

Decreased melatonin levels in postmortem cerebrospinal fluid in relation to aging, Alzheimer's disease, and apolipoprotein E-ع/4/4 genotype. Journal of Endocrinology and Metabolism, 84, 323-327.

Macri, S., Adriani, W., Chiarotti, F., \& Laviola, G. (2002). Risk taking during exploration of a plus-maze is greater in adolescent than in juvenile or adult mice. Animal Behaviour, 64, 541-546.

Magnusson, K.R., Scruggs, B., Aniya, J., Wright, K.C., Ontl, T., Xing, Y., \& Bai, L. (2003). Age-related deficits in mice performing working memory tasks in a water maze. Behavioral Neuroscience, 117, 485-495.

Manev, H., Uz, T., Kharlamov, A., \& Joo, J. (1996). Increased brain damage after stroke or excitotoxic seizures in melatonin-deficient rats. FASEB, 10, 1546-1551.

Marshall, K., Reiter, R.J., Poeggeler, B., Aruomo, O.I., \& Halliwell, B. (1996). Evaluation of the antioxidant activity of melatonin in vitro. Free Radical Biology and Medicine, 21, 307-315.

Matsubara, E., Bryant-Thomas, T., Quinto, J.P., Henry, T.L., Poeggeler, B., Herbert, D., Cruz-Sanchez, G., Chyan, Y., Smith, M.A., Perry, G., Shoji, M., Abe, K., Leone, A., Grundke-Ikbal, I., Wilson, G.L., Ghiso, J., Williams, C., Refolo, L.M., \& Pappolla, M.A. (2003). Melatonin increases survival and inhibits oxidative and amyloid pathology in a transgenic model of Alzheimer's disease. Journal of Neurochemistry, 85, 1101-1108.

Mayo, J.C., Sainz, R.M., Antolin, I., Herrera, F., Martin, V., \& Rodriguez, C. (2002). MelatoninRegulation of antioxidant enzyme gene expression. Cellular and Molecular Life Sciences, 59, 1706-1713.

Melchiorri, D., Reiter, R.J., Attia, A.M., Hara, M., Burgos, A., \& Nistico, G. (1995). Potent protective effect of melatonin on in vivo paraquat-induced oxidative damage in rats. Life Sciences, 56, 83-87.

Melchiorri, D., Reiter, R.J., Sewerynek, E., Chen, L.D., \& Nistico, G. (1995). Melatonin reduces kainate-induced lipid peroxidation in homogenates of different brain regions. FASEB, 9, 1205-1210.

Melchiorri, D., Reiter, R.J., Chen, L.D., Sewerynek, E., \& Nistico, G. (1996). Melatonin affordsprotection against kainate-induced in vitro lipid peroxidation in brain. European Journal_of Pharmacology, 305, 239-242.

Miranda, S., Opazo, C., Larrondo, L.F., Munoz, F.G., Ruiz, F., Leighton, F., Inestrosa, N.C. (2000). The role of oxidative stress in the toxicity induced by amyloid Bpeptide in Alzheimer's disease. Progress in Neurobiology, 62, 633-648. 
Montine, K.S., Kim, P.J., Olson, S.J., Markersbery, W.R., Montine, T.J. (1997). 4hydroxy-2-nonenal pyrrole adducts in human neurodegenerative disease. Journal of Neurosurgery and Experimental Neurology, 56, 866-871.

Nakatomi, H., Kuriu, T., Okabe, S., Yamamato, S., Hatano, O., Kawahara, N., Tamura, A., Kirino, T., \& Nakafuku, M. (2002). Regeneration of hippocampal pyramidal neurons after ischemic brain injury by recruitment of endogenous neural progenitors. Cell, 110, 429-441.

Nava, F., \& Carta, G. (2001). Melatonin reduces anxiety induced by lipopolysaccharide in the rat. Neuroscience Letters, 307, 57-60.

Neville, K., McNaughton, N. (1986). Anxyolitic-like action of melatonin on acquisition but not performance of DRL. Pharmacology, Biochemistry, and Behovior, 24, 1497-1502.

Okatani, Y., Wakatsuki, A., \& Kaneda, C. (2000). Melatonin increases activities of glutathione peroxidase and superoxide dismutase in fetal rat brain. Journal of Pineal Research, 28, 89-96.

Olton, D.S., \& Papas, B.C. (1979). Spatial memory and hippocampal function. Neuropsychologia, 17, 669-682.

Ortiz, G.G., Sanchez-Ruiz, M.Y., Tan, D.X., Reiter, R.J., Benitez-King, G., Beas-Zarate, C. (2001). Melatonin, vitamin $\mathrm{E}$, and estrogen reduce damage induced by kainic acid in the hippocampus: potassium-stimulated GABA release. Journal of Pineal Research, 31, 62-67.

Pappolla, M.A., Sos, M., Omar, R.A., Bick, R.J., Hickson-Bick, D.L.M., \& Reiter, R.J. (1997). Melatonin prevents death of neuroblastoma cells exposed to the Alzheimer amyloid peptide. Journal of Neuroscience, 17, 1683-1690.

Pappolla, M.A., Bozner, P., Soto, C., Shao, H., Robakis, N., Zagorski, M., Frangione, B., \& Ghiso, J. (1998). Inhibition of Alzheimer $\beta$-fibrillogenesis by melatonin. Journal of Biological Chemistry, 273, 7185-7189.

Pappolla, M.A., Chyan, Y.J., Poeggeler, B., Bozner, P., Ghiso, J., LeDoux, S.P., \& Wilson, G.L. (1999). Alzheimer $\beta$ protein mediated oxidative damage of mitochondrial DNA: Prevention by melatonin. Journal of Pineal Research, 27, 226-229.

Pappolla, M.A., Chyan, Y.J., Poeggeler, B., Frangione, B., Wilson, G., Ghiso, J., \& Reiter, R.J. (2000). An assessment of the antioxidant and the antiamyloidogenic properties of melatonin: Implications for Alzheimer's disease. Journal of Neural Transmission, 107, 203-231. 
Pang, S.F., Tang, P.L., Yu, H.S., \& Yip, M.K. (1982). The level of N-acetylserotonin and melatonin in the brain of male rats: diurnal variations and effects of pinealectomy. Journal of Experimental Zoology, 219, 276-286.

Pei, Z., \& Cheung, R.T. (2004). Pretreatment with melatonin exerts anti-inflammatory effects against ischemia/reperfusion injury in a rat middle cerebral artery occlusion stroke model. Journal of Pineal Research, 37, 85-91.

Pellow, S., Chopin, P., File, S.E., \& Briley, M. (1985). Validation of open: closed arm entries in an elevated plus-maze as a measure of anxiety in the rat. Journal of Neuroscience Methods, 14, 149-167.

Perreau-Lenz, S., Kalsbeek, A., Garidou, M., Wortel, J., van der Vliet, J., van Heijningen, C., Simonneaux, V., \& Buijs, R.M. (2003). Suprachiasmatic control of melatonin synthesis in rats: inhibitory and stimulatory mechanisms. European Journal of Neuroscience, 17, 221-228.

Pieri, C., Maria, M., Moroni, F., Redhioni, R., \& Marcheselli, F. (1994). Melatonin: peroxyl radical scavenger more effective than vitamin E. Life Sciences, 55, 271276.

Pierpaoli, W., \& Regelson, W. (1994). Pineal control of aging: Effect of melatonin and pineal grafting on aging mice. Proceedings of the National Academy of Science, 91, 787-791.

Poeggeler, B., Miravalle, L., Zagorski, M.G., Wisniewski, T., Chyan, Y., Zhang, Y., Shao, H., Bryant-Thomas, T., Vidal, R., Frangione, B., Ghiso, J., \& Pappolla, M.A. (2001). Melatonin reverses the profibrillogenic activity of apolipoprotein E4 on the Alzheimer amyloid A $\beta$ peptide. Biochemistry, 40, 14995-15001.

Raghavendra, V., \& Kulkarni, S.K. (2001). Possible antioxidant mechanism in melatonin reversal of aging and chronic ethanol-induced amnesia in plus-maze and passive avoidance memory tasks. Free Radical Biology and Medicine, 30, 595-602.

Rapp, P.R., \& Gallagher, M. (1996). Preserved neuron number in the hippocampus of aged rats with spatial learning deficits. Proceedings of the National Academy of Science, 93, 9926-9930.

Rasmussen, T., Schliemann, T., Sorensen, J.C., Zimmer, J., \& West, M.J. (1996). Memoryimpaired aged rats: No loss of principle hippocampal and subicular neurons. Neurobiology of Aging, 17, 143-147.

Regrigny, O., Dupuis, F., Atkinson, J., Liminana, P., Scalbert, E., Delagrange, P., \& Chillon, J.M. (2001). Cerebral arteriolar structure and function in pinealectomized rats. American Journal of Physiology and Heart Circulatory Physiology, 281, H1476-480. 
Reiter, R.J. (1991). Pineal melatonin: Cell biology of its synthesis and of its physiological interactions. Endocrine Reviews, 12, 151-180.

Reiter, R.J. (1995). Oxidative processes and antioxidative defense mechanisms in the aging brain. FASEB, 9, 526-533.

Reiter, R.J. (1995). The pineal gland and melatonin in relation to aging: A summary of the theories and of the data. Experimental Gerontology, 30, 199-212.

Reiter, R.J. (1996). Functional aspects of the pineal hormone melatonin in combating cell and tissue damage induced by free radicals. European Journal of Endocrinology, $134,-412-420$.

Reiter, R.J., Ortiz, G.G., Monti, M.G., \& Carneiro, R.C. (1997). Cellular and molecular actions of melatonin as an antioxidant. Frontiers of Hormonal Research, 23, 8188 .

Reiter, R.J. (1998). Oxidative damage in the central nervous system: Protection by melatonin.Progress in Neurobiology, 56, 359-384.

Reiter, R.J., Guerrero, J.M., Garcia, J.J., Acuna-Castroviejo, D. (1998). Reactive oxygen Intermediates molecular damage and aging: Relation to melatonin. Annals of the New York Academy of Sciences, 854, 410-454.

Reiter, R.J., Tan, D.X., Kim, S.J., Manchester, L.C. Qi, W., Garcia, J.J., Cabrera, J.C., ElSokkary, G., \& Rouvier-Govary, V. (1999). Augmentation of indices of oxidative damage in life-long melatonin-deficient rats. Mechanisms of Ageing and Development, 10, 157-173.

Rodriguez, C., Mayo, J.C., Sainz, R.M., Antolin, I., Herrera, F., Martin, V., \& Reiter, R.J. (2004). Regulation of antioxidant enzymes: A significant role for melatonin. Journal of Pineal Research, 36, 1-9.

Schmidt, W., \& Reymann, K.G. (2002). Proliferating cells differentiate into neurons in the hippocampal CA1 region of gerbils after global cerebral ischemia. Neuroscience Letters, 334, 153-156.

Schumued, L.C., \& Hopkins, K.J. (2000). Fluoro-Jade B: a high affinity fluorescent marker for the localization of neuronal degeneration. Brain Research, 874, 123130.

Selkoe, D.J. (1996). Amyloid beta-protein and the genetics of Alzheimer's disease. Journal of Biological Chemistry, 271, 18295-18298. 
Selkoe, D.J. (1997). Alzheimer's disease: Genotypes, phenotypes, and treatments. Science, $275,630-631$.

Shukitt-Hale, B., Mouzakis, G., \& Joseph, J.A. (1998). Psychomotor and spatial memory performance in aging male Fischer 344 rats. Experimental Gerontology, 33, 615624.

Siesjo, B.K. (1988): Mechanisms of ischemic brain damage. Critical Care Medicine, 16, 954-963.

Sinha, K., Degaonkar, M.N., Jagannathan, N.R., \& Gupta, Y.K. (2001). Effect of melatonin on ischemia reperfusion injury induced by middle cerebral artery occlusion in rats. European Journal of Pharmacology, 428, 185-192.

Smith, M.A., Tabaton, M., \& Perry, G., (1996). Early contribution of oxidative glycation in Alzheimer disease. Neuroscience Letters, 217, 210-211.

Srinivasan, V. (1999). Melatonin, oxidative stress, and ageing. Current Science, 76, 4654.

Subbarao, K.V., Richardson, J.S., Ang, L.C. (1990). Autopsy samples of Alzheimer's cortex show increased peroxidation in vitro. Journal of Neurochemistry, 55, 342345 .

Sugden, D. (1989). Melatonin biosynthesis in the mammilian pineal gland. Experentia, $45,922-931$.

Tan, D.X., Chen, L., Poeggeler, B., Manchester, L.C., Reiter, R.J. (1993). Melatonin: A potent endogenous hydroxyl radical scavenger. Endocrine Journal, 1, 57-60.

Tan, D.X., Poeggeler, B., Reiter, R.J., Chen, L., Chen. S., Manchester, L.C., \& BarlowWalden, L.R. (1993). The pineal hormone melatonin inhibits DNA-adduct formation induced by the chemical carcinogen safrole in vivo. Cancer Letters, 70, $65-71$.

Tan, D.X., Reiter, R.J., Chen, L., Poeggerler, B., Manchester, L.C., Barlow-Walden, L.R. (1994).Both physiological and pharmacological levels of melatonin reduce DNA adduct formation induced by the carcinogen safrole. Carcinogenesis, 15, 215-218.

Thomas, B., \& Mohanakumar, K.P. (2004). Melatonin protects against oxidative stress caused by 1-methyl-4-phenyl-1,2,3,6-tetrahydropyridine in the mouse nigrostriatum. Journal of Pineal Research, 36, 25-32. 
Torii, K., Uneyama, H., Nishino, H., \& Kondoh, T. (2004). Melatonin suppresses cerebral edema caused by middle cerebral artery occlusion/reperfusion in rats assessed by magnetic resonance imaging. Journal of Pineal Research, 36, 18-24.

Vanecek, J. (1998). Cellular mechanisms of melatonin action. Physiological Reviews, 78, 687-721.

Wall, P.M., \& Messier, C. (2001). Methodological and conceptual issues in the use of the elevated plus-maze as a psychological measurement instrument of animal anxietylike behavior. Neuroscience and Biobehavioral Reviews, 25, 275-286.

Wallace, J.E., Krauter, E.E., \& Campbell, B.A. (1980). Animal models of declining memory in the aged: short-term and spatial memory in the aged rat. Journal of Gerontology, 35, 355-363.

Wei, J., Huang, N.C., \& Quast, M.J. (1997). Hydroxyl radical formation in hyperglycemic rats during middle cerebral artery occlusion/reperfusion. Free Radical Biology and Medicine, 23, 986-995.

West, M.J. (1993). New stereological methods for counting neurons. Neurobiology of Aging, 14, 275-285.

West, M.J., Slomanka, L., \& Gundersen, H.J.G. (1991). Unbiased stereological estimation of the total number of neurons in the subdivisions of the rat hippocampus using the optical fractionator. The Anatomical Record, 231, 482497.

Yoritaka, A., Hattori, N., Uchida, K., Tanaka, M., Stadman, E.R., \& Mizuno, Y. (1996). immunohistochemical detection of 4-hydroxynonenal protein adducts in Parkinson's disease. Proceedings of the National Academy of Sciences, 7, 2696701.

Zhdanova, I.V., Wurtman, R.J., Lynch, H.J., Ives, J.R., Dollins, A.B., Morabito, C., Matheson, J.K., Schomer, D.L. (1995). Sleep-inducing effects of low doses of melatonin ingested in the evening. Clinical Pharmacology Therapeutics, 57, 552558.

Zhou, J.N., Liu, R.L., Kamphorst, W., Hofman, M.A., \& Swaab, D.F. (2003). Early neuropathological Alzheimer's changes in aged individuals are accompanied by decreased cerebrospinal fluid melatonin levels. Journal of Pineal Research, 35, 125-130.

Zini, I., Tomasi, A., Grimaldi, R., Vannani, V., \& Agnati, L.F. (1992). Detection of free radicals during brain ischemia and reperfusion by spin trapping and microdialysis. Neuroscience Letters, 138, 279-282. 
Appendix A1. ANOVA Summary Table for Body Weight

\begin{tabular}{lcccc}
\hline Source & df & MS & F & p \\
\hline Group & 2 & 5682.158 & 1.798 & .185 \\
Error & 27 & 3160.246 & & \\
Total & 30 & & & \\
\hline
\end{tabular}


Appendix A2. ANOVA Summary Tables for Elevated Plus Maze

Total Entries: 2-Months Post-Pinealectomy

\begin{tabular}{lcccc}
\hline Source & df & MS & F & p \\
Group & 2 & 138.133 & .741 & .486 \\
Error & 27 & 186.311 & & \\
Total & 30 & & & \\
\hline
\end{tabular}

Open Entries: 2-Months Post-Pinealectomy

\begin{tabular}{lcccc}
\hline Source & df & MS & F & p \\
\hline Group & 2 & 9.100 & .577 & .568 \\
Error & 27 & 15.770 & & \\
Total & 30 & & & \\
\hline
\end{tabular}

Closed Entries: 2-Months Post-Pinealectomy

\begin{tabular}{lcccc}
\hline Source & df & MS & F & P \\
\hline Group & 2 & 10.000 & .509 & .606 \\
Error & 27 & 19.630 & & \\
Total & 30 & & & \\
& & & & \\
\hline
\end{tabular}


Open Entry Ratio: 2-Months Post-Pinealectomy

\begin{tabular}{lcccc}
\hline Source & df & MS & F & p \\
\hline Group & 2 & 18.302 & .494 & .616 \\
Error & 27 & 37.083 & & \\
Total & 30 & & \\
\hline
\end{tabular}

Open Time Ratio: 2-Months Post-Pinealectomy

\begin{tabular}{lcccc}
\hline Source & df & MS & F & P \\
Group & 2 & 33.427 & .411 & .667 \\
Error & 27 & 81.386 & & \\
Total & 30 & & & \\
\hline
\end{tabular}

Hub Time Ratio: 2-Months Post-Pinealectomy

\begin{tabular}{lcccc}
\hline Source & df & MS & F & p \\
\hline Group & 2 & 111.423 & 3.285 & .050 \\
Error & 27 & 33.917 & & \\
Total & 30 & & & \\
\hline
\end{tabular}


Protected Stretch Attend Ratio: 2-Months Post-Pinealectomy

\begin{tabular}{lcccc}
\hline Source & df & MS & F & P \\
\hline Group & 2 & 683.378 & 2.839 & .076 \\
Error & 27 & 240.691 & & \\
Total & 30 & & & \\
\hline
\end{tabular}

Vertical Stretches: 2-Months Post-Pinealectomy

\begin{tabular}{|c|c|c|c|c|}
\hline Source & df & & $\mathbf{F}$ & $p$ \\
\hline Group & 2 & 57.433 & 2.229 & .127 \\
\hline Error & 27 & 25.770 & & \\
\hline Total & 30 & & & \\
\hline
\end{tabular}

Head Dips: 2-Months Post-Pinealectomy

\begin{tabular}{lcccc}
\hline Source & df & MS & F & P \\
\hline Group & 2 & 31.033 & .385 & .684 \\
Error & 27 & 80.567 & & \\
Total & 30 & & & \\
\hline
\end{tabular}


Total Entries: 7-Months Post-Pinealectomy

\begin{tabular}{lcccc}
\hline Source & df & MS & F & p \\
\hline Group & 2 & 78.433 & .260 & .773 \\
Error & 27 & 301.263 & & \\
Total & 30 & & & \\
\hline
\end{tabular}

Closed Entries: 7-Months Post-Pinealectomy

\begin{tabular}{lcccc}
\hline Source & df & MS & F & p \\
\hline Group & 2 & 9.100 & .248 & .782 \\
Error & 27 & 36.744 & & \\
Total & 30 & & & \\
\hline
\end{tabular}

Open Entries: 7-Months Post-Pinealectomy

\begin{tabular}{lcccr}
\hline Source & df & MS & F & p \\
\hline Group & 2 & 13.900 & 1.149 & .332 \\
Error & 27 & 12.093 & & \\
Total & 30 & & & \\
\hline
\end{tabular}


Open Entry Ratio: 7-Months Post-Pinealectomy

\begin{tabular}{lccccc}
\hline Source & df & MS & F & P \\
\hline Group & 2 & 26.536 & .551 & .583 \\
Error & 27 & 48.154 & & \\
Total & 30 & & & \\
\hline
\end{tabular}

Open Time Ratio: 7-Months Post-Pinealectomy

\begin{tabular}{lcccc}
\hline Source & df & MS & F & P \\
\hline Group & 2 & 45.300 & .701 & .505 \\
Error & 27 & 64.628 & & \\
Total & 30 & & & \\
& & & & \\
\hline
\end{tabular}

Hub Time Ratio: 7-Months Post-Pinealectomy

\begin{tabular}{lcccc}
\hline Source & df & MS & F & p \\
\hline Group & 2 & 15.413 & .158 & .855 \\
Error & 27 & 97.516 & & \\
Total & 30 & & & \\
& & & & \\
\hline
\end{tabular}


Protected Stretch Attend Ratio: 7-Months Post-Pinealectomy

\begin{tabular}{lcccc}
\hline Source & df & MS & F & p \\
\hline Group & 2 & 38.659 & .066 & .936 \\
Error & 27 & 584.839 & & \\
Total & 30 & & \\
\hline
\end{tabular}

Vertical Stretches: 7-Months Post-Pinealectomy

\begin{tabular}{lcccc}
\hline Source & df & MS & F & p \\
\hline Group & 2 & 48.700 & .609 & .551 \\
Error & 27 & 79.959 & & \\
Total & 30 & & \\
\hline
\end{tabular}

Head Dips: 7-Months Post-Pinealectomy

\begin{tabular}{lcccc}
\hline Source & df & MS & F & p \\
\hline Group & 2 & 21.100 & .520 & .601 \\
Error & 27 & 40.611 & & \\
Total & 30 & & \\
\hline
\end{tabular}


Total Entries: 11-Months Post-Pinealectomy

\begin{tabular}{lccccc}
\hline Source & df & MS & F & p \\
\hline Group & 2 & 353.200 & 1.048 & .365 \\
Error & 27 & 337.052 & & \\
Total & 30 & & & \\
\hline
\end{tabular}

Closed Entries: 11-Months Post-Pinealectomy

\begin{tabular}{lcccc}
\hline Source & df & MS & F & p \\
\hline Group & 2 & 67.900 & 1.113 & .343 \\
Error & 27 & 60.981 & & \\
Total & 30 & & & \\
\hline
\end{tabular}

Open Entries: 11-Months Post-Pinealectomy

\begin{tabular}{lcccc}
\hline Source & df & MS & F & p \\
\hline Group & 2 & 1.433 & .754 & .754 \\
Error & 27 & 5.030 & & \\
Total & 30 & & & \\
\hline
\end{tabular}


Open Entry Ratio: 11-Months Post-Pinealectomy

\begin{tabular}{lcccc}
\hline Source & df & MS & F & p \\
\hline Group & 2 & 1.304 & .012 & .988 \\
Error & 27 & 104.645 & & \\
Total & 30 & & & \\
& & & & \\
\hline
\end{tabular}

Open Time Ratio: 11-Months Post-Pinealectomy

\begin{tabular}{lcccc}
\hline Source & df & MS & \multicolumn{1}{c}{ F } & p \\
\hline Group & 2 & 16.112 & .961 & .395 \\
Error & 27 & 16.764 & & \\
Total & 30 & & & \\
\hline
\end{tabular}

Hub Time Ratio: 11-Months Post-Pinealectomy

\begin{tabular}{lcccc}
\hline Source & df & MS & F & p \\
\hline Group & 2 & 72.661 & 1.635 & .214 \\
Error & 27 & 44.434 & & \\
Total & 30 & & & \\
\hline
\end{tabular}


Protected Stretch Attend: 11-Months Post-Pinealectomy

\begin{tabular}{lcccc}
\hline Source & df & MS & \multicolumn{1}{c}{ F } & p \\
\hline Group & 2 & 342.433 & .319 & .729 \\
Error & 27 & 1072.315 & & \\
Total & 30 & & & \\
\hline
\end{tabular}

Vertical Stretches: 11-Months Post-Pinealectomy

\begin{tabular}{lcccc}
\hline Source & df & MS & F & p \\
\hline Group & 2 & 101.033 & 1.668 & .207 \\
Error & 27 & 60.570 & & \\
Total & 30 & & & \\
\hline
\end{tabular}

Head Dips: 11-Months Post-Pinealectomy

\begin{tabular}{lcccc}
\hline Source & df & MS & F & p \\
\hline Group & 2 & 5.700 & .291 & .750 \\
Error & 27 & 19.559 & & \\
Total & 30 & & & \\
\hline
\end{tabular}


Total Entries: 15-Months Post-Pinealectomy

\begin{tabular}{lcccc}
\hline Source & df & MS & F & p \\
\hline Group & 2 & 528.533 & 3.330 & .050 \\
Error & 27 & 158.696 & & \\
Total & 30 & & & \\
\hline
\end{tabular}

Open Entries: 15-Months Post-Pinealectomy

\begin{tabular}{lcccc}
\hline Source & df & MS & F & p \\
\hline Group & 2 & .700 & .574 & .570 \\
Error & 27 & 1.219 & & \\
Total & 30 & & & \\
\hline
\end{tabular}

Closed Entries: 15-Months Post-Pinealectomy

\begin{tabular}{lccccc}
\hline Source & df & MS & F & $\mathbf{p}$ \\
\hline Group & 2 & 117.033 & 3.116 & .061 \\
Error & 27 & 37.559 & & \\
Total & 30 & & & \\
\end{tabular}


Open Entry Ratio: 15-Months Post-Pinealectomy

\begin{tabular}{lcccc}
\hline Source & df & MS & F & p \\
\hline Group & 2 & 64.279 & .847 & .440 \\
Error & 27 & 75.926 & & \\
Total & 30 & & & \\
\hline
\end{tabular}

Open Time Ratio: 15-Months Post-Pinealectomy

\begin{tabular}{lccccc}
\hline Source & df & MS & F & p \\
\hline Group & 2 & 24.577 & 1.068 & .358 \\
Error & 27 & 23.008 & & \\
Total & 30 & & & \\
\hline
\end{tabular}

Hub Time Ratio: 15-Months Post-Pinealectomy

\begin{tabular}{lccccc}
\hline Source & df & MS & F & p \\
\hline Group & 2 & 32.800 & 1.226 & .309 \\
Error & 27 & 26.753 & & & \\
Total & 30 & & & & \\
\hline
\end{tabular}


Protected Stretch Attends: 15-Months Post-Pinealectomy

\begin{tabular}{lrrrrr}
\hline Source & df & MS & F & P \\
\hline Group & 2 & 1877.182 & 1.502 & .241 \\
Error & 27 & 1249.964 & & \\
Total & 30 & & & \\
\end{tabular}

Vertical Stretches: 15-Months Post-Pinealectomy

\begin{tabular}{lcccc}
\hline Source & df & MS & F & $\mathbf{p}$ \\
\hline Group & 2 & 37.900 & 1.083 & .353 \\
Error & 27 & 35.000 & & \\
Total & 30 & & & \\
& & & & \\
\hline
\end{tabular}

Head Dips: 15-Months Post-Pinealectomy

\begin{tabular}{lcccc}
\hline Source & df & MS & F & P \\
Group & 2 & 6.533 & .748 & .483 \\
Error & 27 & 8.737 & & \\
Total & 30 & & & \\
& & & & \\
\hline
\end{tabular}


Total Entries: All Age Groups

\begin{tabular}{lcccc}
\hline Source & df & MS & F & p \\
\hline Group & 2 & 658.358 & 2.678 & .073 \\
Age & 3 & 6834.897 & 27.803 & .000 \\
Group X Age & 6 & 146.647 & .597 & .733 \\
Error & 108 & 245.831 & & \\
Total & 120 & & & \\
\hline
\end{tabular}

\section{Closed Entries: All Age Groups}

\begin{tabular}{lcccr}
\hline Source & df & MS & F \\
\hline Group & 2 & 84.633 & 2.185 & .117 \\
Age & 3 & 651.275 & 16.816 & .000 \\
Group X Age & 6 & 39.800 & 1.028 & .411 \\
Error & 108 & 38.729 & & \\
Total & 120 & & & \\
\hline
\end{tabular}


Open Entries: All Age Groups

\begin{tabular}{lccccc}
\hline Source & df & MS & F & p \\
\hline Group & 2 & 12.758 & 1.496 & .229 \\
Age & 3 & 293.567 & 34.425 & .000 \\
Group X Age & 6 & 4.125 & .484 & .819 \\
Error & 108 & 8.528 & & \\
Total & 120 & & & \\
\hline
\end{tabular}

Open Entry Ratio: All Age Groups

\begin{tabular}{lcccc}
\hline Sources & df & MS & F & p \\
\hline Group & 2 & 33.981 & .511 & .601 \\
Age & 3 & 489.738 & 7.370 & .000 \\
Group X Age & 6 & 25.480 & .383 & .888 \\
Error & 108 & 66.452 & & \\
Total & 120 & & & \\
\hline
\end{tabular}


Open Time Ratio: All Age Groups

\begin{tabular}{lrrrr}
\hline Source & df & MS & F & p \\
\hline Group & 2 & 36.443 & .785 & .459 \\
Age & 3 & 1097.292 & 23.625 & .000 \\
Group X Age & 6 & 27.658 & .595 & .733 \\
Error & 108 & 46.447 & & \\
Total & 120 & & & \\
\hline
\end{tabular}

Hub Time Ratio: All Age Groups

\begin{tabular}{lrrrr}
\hline Source & df & MS & F & p \\
Group & 2 & 144.832 & 2.859 & .062 \\
Age & 3 & 2073.082 & 40.925 & .000 \\
Group X Age & 6 & 29.155 & .576 & .749 \\
Error & 108 & 50.655 & & \\
Total & 120 & & & \\
\hline
\end{tabular}


Protected Stretch Attends: All Age Groups

\begin{tabular}{lrrrr}
\hline Source & df & MS & F & p \\
\hline Group & 2 & 506.119 & .643 & .528 \\
Age & 3 & 15066.853 & 19.146 & .000 \\
Group X Age & 6 & 811.844 & 1.032 & .409 \\
Error & 108 & 786.952 & & \\
Total & 120 & & & \\
\hline
\end{tabular}

Vertical Stretches: All Age Groups

\begin{tabular}{lcccc}
\hline Source & df & MS & F & p \\
\hline Group & 2 & 108.475 & 2.155 & .121 \\
Age & 3 & 807.031 & 16.036 & .000 \\
Group X Age & 6 & 45.531 & .905 & .494 \\
Error & 108 & 50.325 & & \\
Total & 120 & & & \\
\hline
\end{tabular}


Head Dips: All Age Groups

\begin{tabular}{lrrrr}
\hline Source & df & MS & F & p \\
\hline Group & 2 & 23.758 & .636 & .531 \\
Age & 3 & 1197.778 & 32.053 & .000 \\
Group X Age & 6 & 13.536 & .362 & .901 \\
Error & 108 & 37.369 & & \\
Total & 120 & & & \\
\hline
\end{tabular}


Appendix A3. ANOVA Summary Tables for Morris Water Maze

Latency: 2-Months Post-Pinealectomy

\begin{tabular}{lcccc}
\hline Source & df & MS & F & p \\
\hline Group & 2 & 341.461 & .232 & .795 \\
Error & 27 & 1473.020 & & \\
Day & 5 & 5646.965 & 19.994 & .000 \\
Day X Group & 10 & 273.123 & .967 & .475 \\
Error (Day) & 135 & 282.436 & & \\
\hline
\end{tabular}

Distance: 2-Months Post-Pinealectomy

\begin{tabular}{lrrrrr}
\hline Source & df & MS & F & p \\
\hline Group & 2 & 397771.161 & .316 & .732 \\
Error & 27 & 1259347.739 & & \\
Day & 5 & 2970185.245 & 23.716 & .000 \\
Day X Group & 10 & 177297.375 & 1.416 & .180 \\
Error (Day) & 135 & 125240.150 & & \\
\hline
\end{tabular}


Latency: 7-Months Post-Pinealectomy

\begin{tabular}{lrrrr}
\hline Source & df & MS & F & p \\
\hline Group & 2 & 197.143 & .216 & .807 \\
Error & 27 & 911.480 & & \\
Day & 5 & 8442.488 & 48.325 & .000 \\
Day X Group & 10 & 139.525 & .799 & .630 \\
Error (Day) & 135 & 174.702 & & \\
\hline
\end{tabular}

Distance: 7-Months Post-Pinealectomy

\begin{tabular}{lcccc}
\hline Source & df & MS & F & \\
\hline Group & 2 & 120785.167 & .301 & .742 \\
Error & 27 & 401026.318 & & \\
Day & 5 & 351016.473 & 45.430 & .000 \\
Day X Group & 10 & 77035.443 & .997 & .449 \\
Error (Day) & 135 & 77283.959 & & \\
\hline
\end{tabular}


Latency: 11-Months Post-Pinealectomy

\begin{tabular}{lrrrr}
\hline Source & df & MS & F & p \\
\hline Group & 2 & 48.879 & .057 & .944 \\
Error & 27 & 850.440 & & \\
Day & 5 & 12221.588 & 58.092 & .000 \\
Day X Group & 10 & 173.120 & .823 & .607 \\
Error (Day) & 135 & 210.382 & & \\
\hline
\end{tabular}

Distance: 11-Months Post-Pinealectomy

\begin{tabular}{lrrrr}
\hline Source & df & MS & F & p \\
\hline Group & 2 & 43782.846 & .151 & .861 \\
Error & 27 & 290546.206 & & \\
Day & 5 & 4405643.890 & 66.187 & .000 \\
Day X Group & 10 & 12472.691 & .187 & .997 \\
Error (Day) & 135 & 66563.535 & & \\
\hline
\end{tabular}




\section{Latency: All Age Groups}

\begin{tabular}{lrrrr}
\hline Source & df & MS & \multicolumn{2}{c}{ F } \\
\hline Age & 2 & 3463.832 & 3.212 & .045 \\
Group & 2 & 116.310 & .108 & .898 \\
Age X Group & 4 & 235.587 & .218 & .927 \\
Error & 81 & 1078.313 & & \\
Day & 5 & 25284.198 & 113.633 & .000 \\
Day X Age & 10 & 513.421 & 2.307 & .012 \\
Day X Group & 10 & 103.002 & .463 & .913 \\
Day X Age X Group & 20 & 241.383 & 1.085 & .363 \\
Error (Day) & 405 & 222.507 & & \\
\hline
\end{tabular}

Distance: All Age Groups

\begin{tabular}{lrrrr}
\hline Source & df & MS & \multicolumn{1}{c}{ F } \\
\hline Age & 2 & 179251.188 & .276 & .760 \\
Group & 2 & 174962.224 & .269 & .765 \\
Age X Group & 4 & 193688.475 & .298 & .879 \\
Error & 81 & 650306.754 & & \\
Day & 5 & 10722360.50 & 119.541 & .000 \\
Day X Age & 10 & 82242.556 & .917 & .517 \\
Day X Group & 10 & 84668.403 & .944 & .492 \\
Day X Age X Group & 20 & 91068.553 & 1.015 & .442 \\
Error (Day) & 405 & 89695.881 & & \\
\hline
\end{tabular}


Appendix A4. ANOVA Summary Tables For Hippocampal Cell Counts

CA1 Cell Count: 2-Months Post-Pinealectomy

\begin{tabular}{lcccc}
\hline Source & df & MS & F & p \\
\hline Group & 2 & 1229012720.000 & 1.449 & .260 \\
Error & 19 & 848299209.200 & & \\
Total & 22 & & & \\
\hline
\end{tabular}

CA3 Cell Count: 2-Months Post-Pinealectomy

\begin{tabular}{lcccc}
\hline Source & df & MS & F & $\mathbf{p}$ \\
\hline Group & 2 & $1.44 \mathrm{E}+09$ & 5.662 & .026 \\
Error & 9 & $2.54 \mathrm{E}+.08$ & & \\
Total & 12 & & & \\
\hline
\end{tabular}


CA1 Cell Count: 4-Months Post-Pinealectomy

\begin{tabular}{lcccc}
\hline Source & df & MS & F & P \\
\hline Group & 2 & $1.90 \mathrm{E}+10$ & 15.503 & .000 \\
Error & 15 & $1.22 \mathrm{E}+09$ & & \\
Total & 18 & & & \\
\hline
\end{tabular}

\section{CA3 Cell Count: 4-Months Post-Pinealectomy}

\begin{tabular}{lcccc}
\hline Source & df & MS & F & p \\
\hline Group & 2 & $2.52 \mathrm{E}+09$ & 7.001 & .007 \\
Error & 15 & $3.60 \mathrm{E}+08$ & & \\
Total & 18 & & & \\
& & & & \\
\hline
\end{tabular}


CA1 Cell Count: 6-Months Post-Pinealectomy

\begin{tabular}{lcccc}
\hline Source & df & MS & F & p \\
\hline Group & 2 & $1.10 \mathrm{E}+10$ & 15.726 & .000 \\
Error & 15 & $6.97 \mathrm{E}+.08$ & & \\
Total & 18 & & & \\
\hline
\end{tabular}

CA3 Cell Count: 6-Months Post-Pinealectomy

\begin{tabular}{lcccc}
\hline Source & df & MS & F & p \\
\hline Group & 2 & $4.16 \mathrm{E}+09$ & 23.501 & .000 \\
Error & 15 & $1.77 \mathrm{E}+08$ & & \\
Total & 18 & & & \\
\hline
\end{tabular}


CA1 Cell Count: 8-Months Post-Pinealectomy

\begin{tabular}{lcccc}
\hline Source & df & MS & F & p \\
\hline Group & 2 & $2.38 \mathrm{E}+12$ & 48.862 & .000 \\
Error & 14 & $4.86 \mathrm{E}+08$ & & \\
Total & 17 & & & \\
\hline
\end{tabular}

CA3 Cell Count: 8-Months Post-Pinealectomy

\begin{tabular}{lcccc}
\hline Source & df & MS & F & \\
\hline Group & 2 & $3.39 \mathrm{E}+09$ & 5.216 & .020 \\
Error & 14 & $6.49 \mathrm{E}+08$ & & \\
Total & 17 & & & \\
\hline
\end{tabular}


CA1 Cell Count: 10-Months Post-Pinealectomy

\begin{tabular}{lcccc}
\hline Source & df & MS & F & p \\
\hline Group & 2 & $8.94 \mathrm{E}+09$ & 18.094 & .000 \\
Error & 11 & $4.94 \mathrm{E}+08$ & & \\
Total & 14 & & & \\
\end{tabular}

CA3 Cell Count: 10-Months Post-Pinealectomy

\begin{tabular}{lcccc}
\hline Source & df & MS & F & p \\
\hline Group & 2 & $2.59 \mathrm{E}+09$ & 7.466 & .010 \\
Error & 10 & $3.46 \mathrm{E}+08$ & & \\
Total & 13 & & & \\
\hline
\end{tabular}


CA1 Cell Count: 17-Months Post-Pinealectomy

\begin{tabular}{lcccc}
\hline Source & df & MS & F & p \\
\hline Group & 2 & $8.16 \mathrm{E}+09$ & 20.665 & .000 \\
Error & 12 & $3.95 \mathrm{E}+08$ & & \\
Total & 15 & & & \\
\hline
\end{tabular}

CA3 Cell Count: 17-Months Post-Pinealectomy

\begin{tabular}{lcccc}
\hline Source & df & MS & \multicolumn{1}{c}{ F } & \\
\hline Group & 2 & $9.00 \mathrm{E}+08$ & 4.377 & .037 \\
Error & 12 & $2.06 \mathrm{E}+08$ & & \\
Total & 15 & & & \\
\hline
\end{tabular}


CA1 Cell Count: All Age Groups

\begin{tabular}{lcccc}
\hline Source & df & MS & F & p \\
Group & 2 & $4.30 \mathrm{E}+10$ & 59.720 & .000 \\
Age & 5 & $5.77 \mathrm{E}+09$ & 8.007 & .000 \\
Group X Age & 10 & $5.05 \mathrm{E}+09$ & 7.008 & .000 \\
Error & 86 & $7.20 \mathrm{E}+08$ & & \\
Total & 104 & & & \\
\hline
\end{tabular}

CA3 Cell Count: All Age Groups

\begin{tabular}{lcccc}
\hline Source & df & MS & F & p \\
Group & 2 & $1.22 \mathrm{E}+10$ & 36.079 & .000 \\
Age & 5 & $3.91 \mathrm{E}+09$ & 11.550 & .000 \\
Group X Age & 10 & $4.21 \mathrm{E}+08$ & 1.244 & .278 \\
Error & 75 & $3.38 \mathrm{E}+08$ & & \\
Total & 93 & & & \\
\hline
\end{tabular}


CA1 Cell Count: All Sham Age Groups

\begin{tabular}{lrrrr}
\hline Source & df & MS & F & p \\
\hline Age Group & 5 & 2084762986 & 3.430 & .015 \\
Error & 29 & 607768162.7 & & \\
Total & 35 & & & \\
& & & & \\
\hline
\end{tabular}

CA1 Cell Count: All Melatonin Age Groups

\begin{tabular}{lcccc}
\hline Source & df & MS & \multicolumn{1}{c}{ F } & \\
\hline Age Group & 5 & $1.006 \mathrm{E}+10$ & 10.661 & .000 \\
Error & 31 & 943209394 & & \\
Total & 37 & & & \\
& & & & \\
\hline
\end{tabular}

\section{CA1 Cell Count: All Pineal Age Groups}

\begin{tabular}{lrrrr}
\hline Source & df & MS & \multicolumn{1}{c}{ F } & \\
\hline Age Group & 5 & 4053120124 & 6.998 & .000 \\
Error & 26 & 579180986.300 & & \\
Total & 32 & & & \\
& & & & \\
\hline
\end{tabular}


CA3 Cell Count: All Sham Age Groups

\begin{tabular}{lcccc}
\hline Source & df & MS & F & p \\
\hline Age Group & 5 & 2430938457 & 9.656 & .000 \\
Error & 24 & 251745734.1 & & \\
Total & 30 & & & \\
\hline
\end{tabular}

CA3 Cell Count: All Melatonin Age Groups

\begin{tabular}{lcccc}
\hline Source & df & MS & \multicolumn{2}{c}{ F } \\
\hline Age Group & 5 & 1039069131 & 2.595 & .051 \\
Error & 25 & 400448503.5 & & \\
Total & 31 & & & \\
\hline
\end{tabular}

CA3 Cell Count: All Pineal Age Groups

\begin{tabular}{lcccc}
\hline Source & df & MS & F & p \\
\hline Age Group & 5 & 1293948664 & 3.614 & .013 \\
Error & 26 & 358016295.600 & & \\
Total & 32 & & & \\
\hline
\end{tabular}


Appendix A5. ANOVA Summary Tables for Fluoro-Jade B

CA1: 2-Months Post-Pinealectomy

\begin{tabular}{lccccc}
\hline Source & df & MS & F & p \\
\hline Group & 2 & 6.990 & 1.236 & .336 \\
Error & 9 & 5.657 & & \\
Total & 12 & & & \\
\hline
\end{tabular}

CA3: 2-Months Post-Pinealectomy

\begin{tabular}{lcccc}
\hline Source & df & MS & \multicolumn{1}{c}{ F } \\
\hline Group & 2 & 34.195 & 1.510 & .272 \\
Error & 9 & 22.640 & & \\
Total & 12 & & & \\
\hline
\end{tabular}

CA4: 2-Months Post-Pinealectomy

\begin{tabular}{lcccc}
\hline Source & df & MS & F & p \\
\hline Group & 2 & .583 & .186 & .834 \\
Error & 9 & 3.139 & & \\
Total & 12 & & & \\
\hline
\end{tabular}


Dendate Gyrus: 2-Months Post-Pinealectomy

\begin{tabular}{lccccc}
\hline Source & df & MS & F & p \\
\hline Group & 2 & 4.646 & .625 & .557 \\
Error & 9 & 7.431 & & \\
Total & 12 & & & \\
\hline
\end{tabular}

\section{CA1: 4-Months Post-Pinealectomy}

\begin{tabular}{lcccc}
\hline Source & df & MS & F & p \\
\hline Group & 2 & 30.635 & .673 & .534 \\
Error & 9 & 45.514 & & \\
Total & 12 & & & \\
\hline
\end{tabular}

CA3: 4-Months Post-Pinealectomy

\begin{tabular}{lccccc}
\hline Source & df & MS & F & p \\
\hline Group & 2 & 37.112 & .708 & .518 \\
Error & 9 & 52.422 & & \\
Total & 12 & & & \\
\hline
\end{tabular}


CA4: 4-Months Post-Pinealectomy

\begin{tabular}{lcccc}
\hline Source & df & MS & F & $\mathbf{p}$ \\
\hline Group & 2 & 4.938 & 1.634 & .248 \\
Error & 9 & 3.021 & & \\
Total & 12 & & & \\
\hline
\end{tabular}

Dendate Gyrus: 4-Months Post-Pinealectomy

\begin{tabular}{lcccc}
\hline Source & df & MS & F & p \\
\hline Group & 2 & 67.688 & 1.037 & .393 \\
Error & 9 & 65.292 & & \\
Total & 12 & & & \\
\hline
\end{tabular}

CA1: 6-Months Post-Pinealectomy

\begin{tabular}{lcccc}
\hline Source & df & MS & F & \\
\hline Group & 2 & .452 & .696 & .523 \\
Error & 9 & .649 & & \\
Total & 12 & & & \\
\hline
\end{tabular}




\section{CA3: 6-Months Post-Pinealectomy}

\begin{tabular}{lcccc}
\hline Source & df & MS & F & p \\
\hline Group & 2 & .363 & .274 & .767 \\
Error & 9 & 1.327 & & \\
Total & 12 & & & \\
\hline
\end{tabular}

CA4: 6-Months Post-Pinealectomy

\begin{tabular}{lcccc}
\hline Source & df & MS & \multicolumn{1}{c}{ F } \\
\hline Group & 2 & .437 & .851 & .459 \\
Error & 9 & .514 & & \\
Total & 12 & & \\
\hline
\end{tabular}

Dendate Gyrus: 6-Months Post-Pinealectomy

\begin{tabular}{lcccc}
\hline Source & df & MS & F & p \\
\hline Group & 2 & .750 & .434 & .661 \\
Error & 9 & 1.729 & & \\
Total & 12 & & & \\
\hline
\end{tabular}


CA1: 8-Months Post-Pinealectomy

\begin{tabular}{lcccc}
\hline Source & df & MS & F & p \\
\hline Group & 2 & 8.514 & 1.787 & .222 \\
Error & 9 & 4.765 & & \\
Total & 12 & & & \\
\hline
\end{tabular}

CA3: 8-Months Post-Pinealectomy

\begin{tabular}{lccccc}
\hline Source & df & MS & F & p \\
\hline Group & 2 & 8.643 & 1.483 & .278 \\
Error & 9 & 5.828 & & \\
Total & 12 & & & \\
\hline
\end{tabular}

CA4: 8-Months Post-Pinealectomy

\begin{tabular}{lcccr}
\hline Source & df & MS & F & p \\
\hline Group & 2 & $6.250 \mathrm{E}-02$ & .092 & .913 \\
Error & 9 & .681 & & \\
Total & 12 & & & \\
\hline
\end{tabular}


Dendate Gyrus: 8-Month Post-Pinealectomy

\begin{tabular}{lcrrr}
\hline Source & df & MS & F & p \\
\hline Group & 2 & 21.583 & 2.573 & .131 \\
Error & 9 & 8.389 & & \\
Total & 12 & & & \\
\hline
\end{tabular}

CA1: 10-Months Post-Pinealectomy

\begin{tabular}{lccccc}
\hline Source & df & MS & F & p \\
\hline Group & 2 & 45.598 & 1.837 & .214 \\
Error & 9 & 24.824 & & \\
Total & 12 & & & \\
& & & & & \\
\hline
\end{tabular}

CA3: 10-Months Post-Pinealectomy

\begin{tabular}{lcccc}
\hline Source & df & MS & F & p \\
\hline Group & 2 & 33.942 & 1.554 & .263 \\
Error & 9 & 21.840 & & \\
Total & 12 & & & \\
\end{tabular}


CA4: 10-Months Post-Pinealectomy

\begin{tabular}{lcccc}
\hline Source & df & MS & F & p \\
\hline Group & 2 & 4.937 & 1.211 & .342 \\
Error & 9 & 4.076 & & \\
Total & 12 & & & \\
\hline
\end{tabular}

Dendate Gyrus: 10-Months Post-Pinealectomy

\begin{tabular}{lcccc}
\hline Source & df & MS & \multicolumn{1}{c}{ F } \\
\hline Group & 2 & 12.521 & 1.119 & .368 \\
Error & 9 & 11.188 & & \\
Total & 12 & & & \\
\hline
\end{tabular}

CA1: 17-Months Post-Pinealectomy

\begin{tabular}{lcccc}
\hline Source & df & MS & F & p \\
\hline Group & 2 & .767 & .238 & .793 \\
Error & 9 & 3.219 & & \\
Total & 12 & & & \\
\hline
\end{tabular}


CA3: 17-Months Post-Pinealectomy

\begin{tabular}{lcccc}
\hline Source & df & MS & \multicolumn{1}{c}{ F } & p \\
\hline Group & 2 & .174 & .092 & .913 \\
Error & 9 & 1.898 & & \\
Total & 12 & & & \\
\hline
\end{tabular}

CA4: 17-Months Post-Pinealectomy

\begin{tabular}{lcccc}
\hline Source & df & MS & F & p \\
\hline Group & 2 & .437 & .299 & .749 \\
Error & 9 & 1.465 & & \\
Total & 12 & & & \\
\hline
\end{tabular}

Dendate Gyrus: 17-Months Post-Pinealectomy

\begin{tabular}{lcccc}
\hline Source & df & MS & F & p \\
\hline Group & 2 & 11.646 & 1.196 & .346 \\
Error & 9 & 9.736 & & \\
Total & 12 & & & \\
\hline
\end{tabular}


Fluoro-Jade B: All Age Groups and Regions

\begin{tabular}{lcccc}
\hline Source & df & MS & F & p \\
\hline Age & 5 & 89.606 & 7.181 & .000 \\
Group & 2 & 7.541 & .604 & .547 \\
Age X Group & 10 & 47.255 & 3.787 & .000 \\
Error & 270 & 12.478 & & \\
Total & 288 & & & \\
\hline
\end{tabular}


Appendix A6. ANOVA Summary Tables for TBARS Levels

Hippocampus: 3-Months Post-Pinealectomy

\begin{tabular}{lcccc}
\hline Source & df & MS & F & p \\
\hline Group & 2 & .992 & .147 & .865 \\
Error & 16 & 6.760 & & \\
Total & 19 & & & \\
\hline
\end{tabular}

Hippocampus: 20-Months Post-Pinealectomy

\begin{tabular}{lcccc}
\hline Source & df & MS & F & p \\
\hline Group & 2 & 7.805 & 1.198 & .322 \\
Error & 21 & 6.514 & & \\
Total & 24 & & & \\
\hline
\end{tabular}

Cortex: 20-Months Post-Pinealectomy

\begin{tabular}{lcccc}
\hline Source & df & MS & F & \\
\hline Group & 2 & $2.156 \mathrm{E}-02$ & .003 & .997 \\
Error & 21 & 8.598 & & \\
Total & 24 & & & \\
\hline
\end{tabular}


Kidney: 20-Months Post-Pinealectomy

\begin{tabular}{lcccc}
\hline Source & df & MS & F & p \\
\hline Group & 2 & 14.230 & .582 & .568 \\
Error & 21 & 24.464 & & \\
Total & 24 & & & \\
\hline
\end{tabular}

All Regions: 20-Months Post-Pinealectomy

\begin{tabular}{lrrrr}
\hline Source & df & MS & F & p \\
\hline Group & 2 & 12.134 & .896 & .413 \\
Region & 2 & 243.014 & 17.949 & .000 \\
Group X Region & 4 & 3.472 & .256 & .905 \\
Error & 60 & 13.539 & & \\
Total & 69 & & & \\
\hline
\end{tabular}

\section{Hippocampus: Effect of Age}

\begin{tabular}{lcrrr}
\hline Source & df & MS & F & P \\
\hline Group & 2 & 5.341 & .806 & .455 \\
Age & 1 & 41.868 & 6.316 & .017 \\
Group X Age & 2 & 1.772 & .267 & .767 \\
Error & 36 & 6.629 & & \\
Total & 42 & & & \\
\hline
\end{tabular}


Appendix A7. ANOVA Summary Tables For Glutathione Levels

Reduced Glutathione: 3-Months Post-Pinealectomy

\begin{tabular}{lcccc}
\hline Source & df & MS & F & p \\
\hline Group & 2 & 962.100 & .353 & .708 \\
Error & 16 & 2725.002 & & \\
Total & 19 & & & \\
\hline
\end{tabular}

Oxidized Glutathione: 3-Months Post-Pinealectomy

\begin{tabular}{lccccc}
\hline Source & df & MS & F & p \\
\hline Group & 2 & 18.095 & 1.403 & .275 \\
Error & 16 & 12.902 & & \\
Total & 19 & & & \\
\hline
\end{tabular}

GSH to GSSG Ratio: 3-Months Post-Pinealectomy

\begin{tabular}{lcccc}
\hline Source & df & MS & F & p \\
\hline Group & 2 & 2867.200 & 3.726 & .047 \\
Error & 16 & 769.453 & & \\
Total & 19 & & & \\
\hline
\end{tabular}


Reduced Glutathione: 20-Months Post-Pinealectomy

\begin{tabular}{lcccc}
\hline Source & df & MS & F & p \\
\hline Group & 2 & 1445.745 & .477 & .628 \\
Error & 20 & 3031.634 & & \\
Total & 23 & & & \\
\hline
\end{tabular}

Oxidized Glutathione: 20-Months Post-Pinealectomy

\begin{tabular}{lcccc}
\hline Source & df & MS & F & p \\
\hline Group & 2 & 7.509 & .649 & .533 \\
Error & 20 & 11.569 & & \\
Total & 23 & & & \\
\hline
\end{tabular}

GSH to GSSG Ratio: 20-Months Post-Pinealectomy

\begin{tabular}{lcccc}
\hline Source & df & MS & F & P \\
\hline Group & 2 & 33.134 & .382 & .688 \\
Error & 20 & 86.792 & & \\
Total & 23 & & & \\
\hline
\end{tabular}


Reduced Glutathione: All Ages

\begin{tabular}{lcccc}
\hline Source & df & MS & F & p \\
\hline Age & 1 & 23209.066 & 5.403 & .026 \\
Group & 2 & 2650.265 & .617 & .545 \\
Age X Group & 2 & 5090.265 & 1.185 & .317 \\
Error & 37 & 4295.785 & & \\
Total & 43 & & & \\
\hline
\end{tabular}

Oxidized Glutathione: All Ages

\begin{tabular}{lcccc}
\hline Source & df & MS & F & p \\
\hline Age & 1 & 1.217 & .103 & .750 \\
Group & 2 & 17.280 & 1.460 & .245 \\
Age X Group & 2 & 10.805 & .913 & .410 \\
Error & 37 & 11.833 & & \\
Total & 43 & & & \\
\hline
\end{tabular}


GSH to GSSG Ratio: 20-Months Post-Pinealectomy

\begin{tabular}{lcccc}
\hline Source & df & MS & F & p \\
\hline Age & 1 & 6266.748 & 11.505 & .002 \\
Group & 2 & 3337.654 & 6.127 & .005 \\
Age X Group & 2 & 2608.756 & 4.789 & .014 \\
Error & 37 & 544.703 & & \\
Total & 43 & & & \\
\hline
\end{tabular}

